\author{
Universidade de São Paulo \\ Instituto de Física
}

\title{
Simulação Computacional de Propriedades Dinâmicas de Heteroestruturas Semicondutoras
}

\author{
Thiago Luiz Chaves de Melo
}

Orientadora: Profa. Dra. Euzi Conceição Fernandes da Silva

Dissertação de mestrado apresentada ao Instituto de Física da Universidade de São Paulo, como requisito parcial para a obtenção do título de Mestre em Ciências.

Banca Examinadora:

Profa. Dra. Lucy V. C. Assali - Presidente da banca em substituição à orientadora (IFUSP)

Prof. Dr. Ronaldo Rodrigues Pelá (ITA)

Prof. Dr. Sandro Martini (Universidade São Judas Tadeu) 


\section{FICHA CATALOGRÁFICA \\ Preparada pelo Serviço de Biblioteca e Informação do Instituto de Física da Universidade de São Paulo}

Melo, Thiago Luiz Chaves de

Simulação computacional de propriedades dinâmicas de heteroestruturas semicondutoras. São Paulo, 2018.

Dissertação (Mestrado) - Universidade de São Paulo. Instituto de Física. Depto. de Física dos Materiais e Mecânica.

Orientador: Profa. Dra. Euzi Conceição Fernandes da Silva.

Área de Concentração: Materiais Semicondutores - Heteroestruturas Semicondutoras.

Unitermos: 1. Heteroestruturas semicondutoras; 2. Fotodetectores; 3. Simulação computacional; 4. Fotocorrente; 5. Absorção. 


\title{
University of São Paulo \\ Physics Institute
}

\section{Computational Simulation of Dynamical Properties of Semiconductor Heterostructures}

\author{
Thiago Luiz Chaves de Melo
}

Supervisor: Prof. Dr. Euzi Conceição Fernandes da Silva

Dissertation submitted to the Physics Institute of the University of São Paulo in partial fulfillment of the requirements for the degree of Master of Science.

Examining Committee:

Prof. Dr. Lucy V. C. Assali - Committee President substituting the Supervisor (IFUSP)

Prof. Dr. Ronaldo Rodrigues Pelá (ITA)

Prof. Dr. Sandro Martini (São Judas Tadeu University) 
Dedico este trabalho à minha família. 


\section{Agradecimentos}

Agradeço em primeiro lugar a minha amiga Euzi por ter me dado uma oportunidade lá no início, em 2012, quando eu ainda estava na graduação e era o mais improvável dos alunos deste instituto. Em segundo lugar agradeço a minha orientadora Profa. Dra. Euzi Conceição Fernandes de Silva, por ter me ensinado praticamente tudo o que sei sobre ciência e muito do que sei sobre a vida. Agradeço acima de tudo por sua paciência e dedicação.

Agradeço a minha esposa Camila Takemoto Bertolini por sempre estar ao meu lado em todos os momentos destes (até aqui) 12 anos juntos e por me ajudar a ser uma pessoa melhor dia após dia.

Agradeço a Profa. Dra. Lucy V. C. Assali por ter sido presidente da banda em substituição à minha orientadora e por ter me ajudado na reta final com as correções.

Agradeço a todos os meus amigos do Instituto de Física da USP que contribuíram das mais variadas formas para minha formação não só acadêmica, mas como cidadão.

Agradeço ao apoio financeiro do Conselho Nacional de Desenvolvimento Científico e Tecnológico ( $\mathrm{CNPq}$ ) sem o qual este trabalho levaria muito mais tempo para se concluído. 

"Quem cede ganha."

Luis Carlos de Melo,

meu pai. 



\section{Resumo}

Neste trabalho desenvolvemos rotinas computacionais em Python para o cálculo de propriedades dinâmicas (espectros de fotocorrente e absorção) de heteroestruturas semicondutoras baseadas em Dinâmica Quântica. Em uma primeira etapa do desenvolvimento do projeto, a formulação baseada na evolução temporal das soluções da equação de Schrödinger dependente do tempo foi aplicada a sistemas com soluções analíticas conhecidas ou com resultados já reportados na literatura. Devido à excelente concordância entre nossos dados e aqueles já conhecidos, em uma etapa seguinte, foram calculadas as energias de transição observadas em espectros de fotoluminescência para poços quânticos de InGaAs/GaAs, crescidos por MBE, levando-se em conta os efeitos de tensão e segregação de átomos de índio. Na continuidade do projeto, especial atenção foi dada ao desenvolvimento de estratégias para calcular os espectros de absorção e fotocorrente para dispositivos do Estado Sólido. O conjunto de resultados apresentados neste trabalho demonstra que a metodologia desenvolvida é precisa e pode ser utilizada com baixo custo computacional para o modelamento de heteroestruturas semicondutoras mais complexas, que servem de base para o desenvolvimento de dispositivos optoeletrônicos.

Palavras-chaves: dinâmica quântica. fotocorrente. espectro de absorção. heteroestruturas semicondutoras. segregação de átomos de índio. 



\section{Abstract}

In this work we developed computational routines in Python for the calculation of the dynamic properties (spectrum of photocurrent and absorption) of semiconductor heterostructures based on Quantum Dynamics Theory. In a first stage of the development of the project the formulation based on the time evolution of the solutions of the time dependent Schrödinger equation was applied to systems with known analytical solutions or results already reported in the literature. Due to the excellent agreement between our data and those already known, in the next stage the transition energies observed in photoluminescence spectra for InGaAs/GaAs quantum wells, grown by MBE, were calculated taking into account the effects of stress and segregation of indium atoms. In the continuity of the project, special attention was given to the development of strategies to calculate absorption and photocurrent spectra for solid state devices. The set of results presented in this work demonstrates that the methodology developed is accurate and can be used with low computational cost for the modeling of more complex semiconductor heterostructures, which are used for the development of optoelectronic devices.

Key-words: quantum dynamics. photocurrent. absorption spectra. semiconductor heterostructures. indium atoms segregation. 



\section{Lista de abreviaturas e siglas}

$\mathrm{Al}$

$\mathrm{Al}_{x} \mathrm{Ga}_{1-x} \mathrm{As}$ Arseneto de gálio alumínio com fração $x$ de alumínio e $1-x$ de gálio

AlAs Arseneto de alumínio

As

CBE

CBO Conduction Band Offset

DFT Transformada Discreta de Fourier, do inglês Discrete Fourier Transform

ESDT Equação de Schrödinger Dependente do Tempo

ESIT Equação de Schrödinger Independente do Tempo

FFT Transformada Rápida de Fourier, do inglês Fast Fourier Transform

Ga

GaAs
Gálio

Arseneto de gálio
HS

Heteroestrutura Semicondutora

In Índio

InAs Arseneto de índio

InGaAs Arseneto de gálio índio

$\operatorname{In}_{x} \mathrm{Ga}_{1-x} \mathrm{As}$ Arseneto de gálio índio com fração $x$ de índio e $1-x$ de gálio

ITP Propagação em Tempo Imaginário, do inglês Imaginary Time Propagation

MBE Molecular Beam Epitaxy

MOCVD Metalorganic Chemical Vapour Deposition

RHEED Reflection High-Energy Electron Diffraction

SI

Sistema Internacional

UA

Unidades Atômicas

VBO
Valence Band Offset 



\section{Sumário}

1 Introdução . . . . . . . . . . . . . . 13

2 Dinâmica Quântica . . . . . . . . . . . . . . . . . . . . . . . 15

2.1 Evolução Temporal . . . . . . . . . . . . . . . . . . . . . . 15

2.2 A Equação de Schrödinger Independente do Tempo . . . . . . . . . . . . . 17

2.3 Propriedades . . . . . . . . . . . . . . . . . . . . . 20

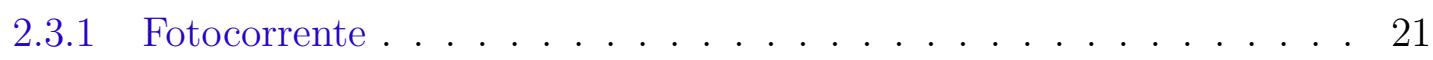

2.3.2 Absorção Intersubbanda . . . . . . . . . . . . . . . . . . . . 22

2.3.2.1 Interação Radiação-Matéria . . . . . . . . . . . . . . . 24

2.3.2.2 Espectro de Absorção . . . . . . . . . . . . . . . . 26

3 Heteroestruturas Semicondutoras . . . . . . . . . . . . . . . . . 31

3.1 Aproximação da Massa Efetiva em Materiais Semicondutores . . . . . . . . 32

3.2 Poços Quânticos Crescidos por MBE . . . . . . . . . . . . . . 36

4 Modelagem Numérica da Equação de Schrödinger . . . . . . . . . . . . . . 39

4.1 Método das Diferenças Finitas . . . . . . . . . . . . . . . . . 39

4.1 .1 Métodos de Runge-Kutta . . . . . . . . . . . . . . . . . 41

4.1 .2 Método de Crank-Nicolson . . . . . . . . . . . . . . . . . . 43

4.1 .3 Estabilidade . . . . . . . . . . . . . . . . . . 44

4.1.4 Massa Efetiva com Dependência Espacial . . . . . . . . . . . . . . 45

4.2 O Método Pseudo-Espectral . . . . . . . . . . . . . . . . . 46

4.2 .1 Etapas para Evolução Temporal . . . . . . . . . . . . . . . . . 48

4.2 .2 Discretização do Espaço e Função de Onda Inicial . . . . . . . . . . . 49

4.2 .3 Transformada Discreta de Fourier . . . . . . . . . . . . . . . 50

4.3 Cálculo de Autovalores e Autovetores . . . . . . . . . . . . . . . . 52

4.3.1 Evolução em Tempo Imaginário: Função de Onda do Estado Fundamental . . . . . . . . . . . . . . . . . . . 52

4.3 .2 O Método da Iteração Inversa . . . . . . . . . . . . . . . . . 53

5 Validação dos Métodos Numéricos Através de Problemas com Solução Conhecida .......................... 55

5.1 Evolução Temporal: Pacote de Onda Plana . . . . . . . . . . . . . . . 55

5.2 Cálculo de Níveis de Energia: Oscilador Harmônico Quântico . . . . . . . . 61

5.3 Cálculo de Níveis de Energia: Poço Quântico . . . . . . . . . . . . . . . . . 64

5.4 Cálculo de Níveis de Energia: Poço Duplo Assimétrico . . . . . . . . . . . . 68 
5.5 Fotocorrente e Absorção em Poço Quântico de GaAs com Barreiras de AlGaAs

6 Efeitos da Tensão e Segregação do Índio (In) em Poços Quânticos de InGaAs/GaAs . . . . . . . . . . . . . . . . . . . . . 77

6.1 Influência da Tensão Elástica . . . . . . . . . . . . . . . . . . 77

6.2 Parâmetros e Ilustração das Correções Induzidas pela Tensão . . . . . . . . 79

6.3 Segregação de In em Poços de InGaAs Crescidos Sobre Substratos de GaAs 81

6.4 Cálculo das Energias de Transição de PL do Poço de InGaAs/ GaAs a partir de Modelagem Numérica . . . . . . . . . . . . . . 83

7 Conclusão . . . . . . . . . . . . . . . . . . . . . 89

Referências ............................. 93

ANEXO A Algoritmos ........................... 97

A.1 Evolução Temporal: Pacote de Onda Plana . . . . . . . . . . . . . . . . . 97

A.2 Cálculo de Níveis de Energia: Oscilador Harmônico Quântico . . . . . . . . . 104

A.3 Cálculo de Níveis de Energia: Poço Quântico . . . . . . . . . . . . . . . . . 107

A.4 Cálculo de Níveis de Energia: Poço Duplo Assimétrico . . . . . . . . . . . . . 115

A.5 Fotocorrente e Absorção em Poço Quântico de GaAs com Barreiras de AlGaAs . . . . . . . . . . . . . . . . . . . . 122

A.6 Efeitos da Tensão e Segregação do In em Poços Quânticos de InGaAs/GaAs134

ANEXO B Bibliotecas ............................... 141

B.1 Constantes . . . . . . . . . . . . . . . . . . . . 141

B.2 Database . . . . . . . . . . . . . . . . . . . . . . . 142

B.3 Utilidades . . . . . . . . . . . . . . . . . . . . . . . . . . . . 148

B.4 Onda Plana . . . . . . . . . . . . . . . . . . . . . . 157

B.5 Oscilador Harmônico Quântico . . . . . . . . . . . . . . . . . . 165

B.6 Poço Quântico . . . . . . . . . . . . . . . . . . . . . . . . . 177

B.7 Potencial do Poço com Dupla Barreira . . . . . . . . . . . . . . . . . . . 185 


\section{Introdução}

Os avanços na tecnologia de fabricação de materiais semicondutores permitem que atualmente construam-se dispositivos com dimensões da ordem de apenas alguns nanômetros. Desta forma, na medida em que os circuitos eletrônicos tornam-se cada vez menores, as teorias clássicas ou semi-clássicas tornam-se inadequadas para analisar as propriedades dinâmicas destes sistemas. Uma vez que as dimensões características dos dispositivos eletrônicos já são comparáveis com o comprimento de onda dos elétrons com energias da ordem de meV até uns poucos eV, os cálculos teóricos das propriedades dinâmicas devem basear-se no formalismo da equação de Schrödinger dependente do tempo (ESDT), isto é, na solução da equação $i \hbar \partial \psi(\vec{r}, t) / \partial t=H \psi(\vec{r}, t)$.

Tradicionalmente, a Química Quântica foi a área científica que mais se dedicou ao desenvolvimento de procedimentos numéricos para a solução da ESDT para o estudo dos processos dinâmico-quânticos em átomos e moléculas. Podemos citar por exemplo os métodos baseados no Split-Operator [1, 2, 3], na expansão de Chebyshev [4] ou no algorítimo iterativo de Lanczos [5]. Embora precisos, estes procedimentos rigorosos são ainda limitados a sistemas moleculares com alguns poucos átomos.

Recentemente, dados os progressos da nanotecnologia na área dos materiais semicondutores, estes métodos da Química Quântica estão também sendo adaptados para o estudo da resposta temporal dos dispositivos do Estado Sólido contendo poços, fios ou pontos quânticos. Estas simulações são importantes para a análise do desempenho dos dispositivos porque são flexíveis e menos dispendiosas do que as experiências. Elas também são importantes para projetar e caracterizar as novas gerações de dispositivos eletrônicos e este é o objetivo do presente projeto. Demos início à implantação deste tipo de cálculo em nosso grupo de pesquisa com a intenção de estudar diferentes processos dinâmicos nos dispositivos semicondutores em desenvolvimento no Laboratório de Novos Materiais Semicondutores (LNMS) do IFUSP. Estamos interessados no cálculo do espectro de absorção, na fotocorrente, na corrente no escuro, na corrente de tunelamento, entre outros processos dinâmicos em heteroestruturas semicondutoras, que representam características importantes para o aprimoramento do desempenho de vários dispositivos do Estado Sólido em desenvolvimento no LNMS.

Assim sendo, nesta dissertação, avaliamos abordagens numéricas para lidar com o problema de simular o comportamento de dispositivos do Estado Sólido. Em especial, objetivamos o cálculo dos níveis de energia quantizados que surgem dentro destes dispositivos, bem como a corrente elétrica resultante devida a absorção de fótons, também chamada de fotocorrente, e o cálculo de espectros de absorção. 
Esta dissertação foi dividida em 7 capítulos. No Capítulo 2 apresentamos os conceitos de Mecânica Quântica que direcionam e fundamentam a compreensão dos resultados numéricos obtidos no restante do trabalho. No Capítulo 3 descrevemos algumas características de poços quânticos de materiais semicondutores. No Capítulo 4 apresentamos métodos numéricos utilizados no cálculo de autovalores e autovetores, bem como métodos para evolução temporal de sistemas quânticos unidimensionais. No Capítulo 5 aplicamos os métodos expostos no Capítulo 4 a sistemas com solução analítica como o oscilador harmônico simples e o poço quadrado finito. Também comparamos nossos resultados com outros trabalhos reportados na literatura.[1,6] No Capítulo 6 aplicamos os métodos numéricos para o cálculo dos níveis de energia de uma amostra contendo vários poços de InGaAs/GaAs crescida no sistema de MBE (Molecular Beam Epitaxy) do LNMS. No Capítulo 7 apresentamos nossas conclusões. Para finalizar, incluímos nos anexos os códigos computacionais desenvolvidos para todos os sistemas analisados. Também é possível acessar os códigos através do endereço https://github.com/thiagolcmelo/mestrado. O intuito é deixar estes programas documentados para todos que têm interesse neste tipo de cálculo, em especial para os integrantes do LNMS. 


\section{Dinâmica Quântica}

A proposta de nosso trabalho contempla o cálculo de grandezas físicas importantes para a caracterização e melhoria do desempenho de dispositivos semicondutores. Nossa intenção é investigar heteroestruturas semicondutoras destinadas à produção de fotodetectores de radiação que operam na faixa de comprimentos de onda de 2 a $20 \mu \mathrm{m}$. Duas propriedades físicas de grande importância para o desempenho dos fotodetectores são a fotocorrente e a absorção, pois estão relacionadas com a eficiência e com a faixa de operação destes dispositivos. Tanto uma quanto a outra podem ser obtidas através do conhecimento dos níveis quânticos de confinamento e da forma como eles se modificam no tempo sob ação de uma força externa, como a aplicação de um campo eletromagnético.

As dimensões típicas com as quais iremos lidar serão da ordem de algumas centenas de angstroms, muito menores do que o comprimento de onda da radiação que nos interessa, regime conhecido como mesoscópico.[7] Assim sendo, a formulação teórica adequada para a descrição destes dispositivos, devido às suas dimensões, requer o uso da Mecânica Quântica e da Dinâmica Quântica para o cálculo de suas propriedades dinâmicas. Nesse regime, ocorre quantização em pelo menos uma das dimensões do sistema.

Neste capítulo introduzimos alguns procedimentos e resultados provenientes da Teoria Quântica que nos serão úteis nos capítulos subsequentes. Apresentamos alguns fenômenos gerais relacionados à evolução temporal de estados quânticos e particularizamos estes fenômenos para o espaço das coordenadas. Na sequência, apresentamos os procedimentos relacionados ao cálculo das propriedades físicas que estamos interessados.

\subsection{Evolução Temporal}

Um único elétron dentro de uma heteroestrutura semicondutora pode ser descrito por um vetor $|\Psi ; t\rangle$ do espaço de Hilbert de dimensão infinita que representa o sistema. Por hora vamos deixar de lado que $|\Psi ; t\rangle$ descreve um elétron e vamos fazer algumas considerações puramente algébricas a respeito deste objeto, ao qual iremos nos referir como estado.

A forma como o estado $|\Psi ; t\rangle$ evolui no tempo, de um instante inicial $t_{\mathrm{i}}$ até um instante final $t_{\mathrm{f}}$, pode ser expressa através da ação de um operador unitário $\mathbf{U}\left(t_{\mathrm{f}}, t_{\mathrm{i}}\right)$ sobre o estado no instante $t_{\mathrm{i}}$

$$
\left|\Psi ; t_{\mathrm{f}}\right\rangle=\mathbf{U}\left(t_{\mathrm{f}}, t_{\mathrm{i}}\right)\left|\Psi ; t_{\mathrm{i}}\right\rangle
$$

No limite em que $t_{\mathrm{f}} \rightarrow t_{\mathrm{i}}$, o operador $\mathbf{U}\left(t_{\mathrm{f}}, t_{\mathrm{i}}\right)$ deve ser reduzido à identidade a menos de 
uma fase, ou seja

$$
\lim _{d t \rightarrow 0} \mathbf{U}\left(t_{\mathrm{i}}+d t, t_{\mathrm{i}}\right)=e^{-i \boldsymbol{\Omega} d t}=\mathbb{1}-i \boldsymbol{\Omega} d t+\mathcal{O}\left(d t^{2}\right),
$$

onde $\Omega$ é um operador hermitiano que pode ter dependência temporal ${ }^{1}$. Uma das vantagens de o operador $\mathbf{U}\left(t_{\mathrm{f}}, t_{\mathrm{i}}\right)$ ser unitário é que seu operador adjunto $\mathbf{U}^{\dagger}\left(t_{\mathrm{f}}, t_{\mathrm{i}}\right)$ retrocede ou involui o estado do instante $t_{\mathrm{f}}$ até o instante $t_{\mathrm{i}}$

$$
\left|\Psi ; t_{\mathrm{i}}\right\rangle=\mathbf{U}^{\dagger}\left(t_{\mathrm{f}}, t_{\mathrm{i}}\right)\left|\Psi ; t_{\mathrm{f}}\right\rangle=\mathbf{U}^{\dagger}\left(t_{\mathrm{f}}, t_{\mathrm{i}}\right) \mathbf{U}\left(t_{\mathrm{f}}, t_{\mathrm{i}}\right)\left|\Psi ; t_{\mathrm{i}}\right\rangle=\mathbb{1}\left|\Psi ; t_{\mathrm{i}}\right\rangle=\left|\Psi ; t_{\mathrm{i}}\right\rangle .
$$

Utilizando o operador hamiltoniano $\mathbf{H}$ dividido pela constante reduzida de Planck $\hbar$ no lugar do operador $\Omega$ da Eq. (2.2) é possível mostrar que o operador de evolução temporal obedece a relação [8]

$$
i \hbar \frac{\partial}{\partial t} \mathbf{U}\left(t, t_{\mathrm{i}}\right)=\mathbf{H} \mathbf{U}\left(t, t_{\mathrm{i}}\right)
$$

que multiplicada em ambos os lados por $\left|\Psi ; t_{i}\right\rangle$,

$$
i \hbar \frac{\partial}{\partial t} \mathbf{U}\left(t, t_{\mathrm{i}}\right)\left|\Psi ; t_{\mathrm{i}}\right\rangle=\mathbf{H} \mathbf{U}\left(t, t_{\mathrm{i}}\right)\left|\Psi ; t_{\mathrm{i}}\right\rangle
$$

resulta na equação de Schrödinger

$$
i \hbar \frac{\partial}{\partial t}|\Psi ; t\rangle=\mathbf{H}|\Psi ; t\rangle
$$

Quando o operador hamiltoniano não possui uma dependência temporal, se escolhermos que em $t_{\mathrm{i}}=0$ o estado do sistema é dado por $|\Psi ; 0\rangle$, a Eq. (2.6) possui solução da forma:

$$
|\Psi ; t\rangle=\exp \left(-\frac{i \mathbf{H} t}{\hbar}\right)|\Psi ; 0\rangle
$$

É possível expandir $|\Psi ; t\rangle$ na base $\{|\mathrm{n}\rangle\}$ dos autovetores de $\mathbf{H}$, ou seja,

$$
|\Psi ; t\rangle=\mathbb{1} \exp \left(-\frac{i \mathbf{H} t}{\hbar}\right)|\Psi ; 0\rangle=\sum_{\mathrm{n}}|\mathrm{n}\rangle\left\langle\mathrm{n}\left|\exp \left(-\frac{i \mathbf{H} t}{\hbar}\right)\right| \Psi ; 0\right\rangle .
$$

Esta expansão é interessante pois a propriedade

$$
f(\mathbf{A})=\sum_{\mathrm{a}} f(\mathrm{a})|\mathrm{a}\rangle\langle\mathrm{a}|
$$

válida para um operador Hermitiano $\mathbf{A}$, com $\mathbf{A}|\mathrm{a}\rangle=\mathrm{a}|\mathrm{a}\rangle$, pode ser utilizada juntamente com a completeza da base $\{|\mathrm{n}\rangle\}$ para escrever

$$
|\Psi ; t\rangle=\sum_{\mathrm{n}} a_{\mathrm{n}}|n\rangle \exp \left(-\frac{i E_{\mathrm{n}} t}{\hbar}\right)
$$

\footnotetext{
$\overline{1 \quad \text { Neste caso devendo ser avaliado em } t}=t_{\mathrm{i}}$.
} 
onde $a_{\mathrm{n}}=\langle\mathrm{n} \mid \Psi ; 0\rangle$ e $\mathbf{H}|\mathrm{n}\rangle=E_{\mathrm{n}}|\mathrm{n}\rangle$.

A Eq. (2.10) permite escrever o estado $|\Psi ; t\rangle$ na representação das coordenadas multiplicando ambos os lados por $\langle\mathbf{r}|$

$$
\Psi(\mathbf{r}, t) \equiv\langle\mathbf{r} \mid \Psi ; t\rangle=\sum_{\mathrm{n}} a_{\mathrm{n}}\langle\mathbf{r} \mid \mathrm{n}\rangle \exp \left(-\frac{i E_{\mathrm{n}} t}{\hbar}\right)
$$

além de evidenciar que apesar de a evolução temporal ocorrer individualmente em cada um dos autovetores do operador hamiltoniano, a evolução temporal do estado $|\Psi ; t\rangle$ como um todo depende de cada coeficiente $a_{\mathrm{n}}=\langle\mathrm{n} \mid \Psi ; 0\rangle$. A importância disso se torna mais clara quando notamos que se $|\Psi ; 0\rangle$ for composto de um único autovetor $|\mathrm{k}\rangle$, o valor esperado de qualquer observável, por exemplo x, é independente do tempo

$$
\begin{aligned}
\langle\mathbf{x}\rangle(t) & =\langle\Psi ; t|\mathbf{x}| \Psi ; t\rangle=\left[\langle\mathrm{k}| \exp \left(+\frac{i E_{\mathrm{k}} t}{\hbar}\right)\right] \mathbf{x}\left[|\mathrm{k}\rangle \exp \left(-\frac{i E_{\mathrm{k}} t}{\hbar}\right)\right] \\
& =\langle\mathrm{k}|\mathbf{x}| \mathrm{k}\rangle=\langle\Psi ; 0|\mathbf{x}| \Psi ; 0\rangle=\langle\mathbf{x}\rangle(0),
\end{aligned}
$$

ao passo que qualquer combinação do tipo $|\Psi ; 0\rangle=a_{\mathrm{i}}|\mathrm{i}\rangle+a_{\mathrm{j}}|\mathrm{j}\rangle$ já implica a existência de uma dinâmica

$$
\begin{aligned}
\langle\mathbf{x}\rangle(t) & =\left[a _ { \mathrm { i } } ^ { * } \left\langle\mathrm{i}\left|e^{\frac{i E_{\mathrm{i}} t}{\hbar}}+a_{\mathrm{j}}^{*}\langle\mathrm{j}| e^{\frac{i E_{\mathrm{j}} t}{\hbar}}\right] \mathbf{x}\left[a_{\mathrm{i}}|\mathrm{i}\rangle e^{-\frac{i E_{\mathrm{i}} t}{\hbar}}+a_{\mathrm{j}}|\mathrm{j}\rangle e^{-\frac{i E_{\mathrm{j}} t}{\hbar}}\right]\right.\right. \\
& =\left|a_{\mathrm{i}}\right|^{2}\langle\mathrm{i}|\mathbf{x}| \mathrm{i}\rangle+a_{\mathrm{i}}^{*} a_{\mathrm{j}}\langle\mathrm{i}|\mathbf{x}| \mathrm{j}\rangle e^{-i \omega_{\mathrm{ij}} t}+a_{\mathrm{i}} a_{\mathrm{j}}^{*}\langle\mathrm{j}|\mathbf{x}| \mathrm{i}\rangle e^{i \omega_{\mathrm{ij}} t}+\left|a_{\mathrm{j}}\right|^{2}\langle\mathrm{j}|\mathbf{x}| \mathrm{j}\rangle
\end{aligned}
$$

dependente do valor de $\omega_{\mathrm{ij}}=\left(E_{\mathrm{i}}-E_{\mathrm{j}}\right) / \hbar$.

A expansão de um estado nos autovetores do hamiltoniano do sistema leva a uma forma muito eficiente de obter a evolução temporal, dada pela Eq. (2.10), que recebe o nome de propagação espectral.[9] Entretanto, quando o hamiltoniano é dependente do tempo, existe energia entrando e/ou saindo do sistema, de modo que a Eq. (2.7) deixa de ser válida, juntamente com a Eq. (2.10).

Nos próximos capítulos utilizaremos técnicas que nos permitem tratar hamiltonianos com dependência temporal de forma estática para pequenos intervalos de tempo, desde que algumas condições sejam obedecidas, como por exemplo a Eq. (4.41). O importante a ser observado aqui é que quase sempre estaremos evoluindo implicitamente e individualmente cada autovetor que faz parte da combinação linear que compõe o estado quântico em questão.

\subsection{A Equação de Schrödinger Independente do Tempo}

A evolução temporal do estado quântico $|\Psi ; t\rangle$ na representação das coordenadas $\Psi(\mathbf{r}, t)=\langle\mathbf{r} \mid \Psi ; t\rangle$ de uma partícula de massa $m$ precisa satisfazer a ESDT:

$$
i \hbar \frac{\partial}{\partial t} \Psi(\mathbf{r}, t)=\mathbf{H} \Psi(\mathbf{r}, t),
$$


onde o operador hamiltoniano $\mathbf{H}$ é a soma dos operadores energia potencial $\mathbf{V}(\mathbf{r}, t)$ e energia cinética $\mathbf{T}$ da partícula:

$$
\mathbf{T}=-\frac{\hbar^{2}}{2 m} \vec{\nabla}^{2}
$$

A solução da Eq. (2.14) dá a função de onda $\Psi(\mathbf{r}, t)$ que carrega toda a informação sobre a distribuição probabilística do sistema e encontrá-la é uma tarefa fundamental na Mecânica Quântica.

Vamos daqui em diante nos limitar ao tratamento de $\Psi(\mathbf{r}, t)$ somente na direção $z$, nos valendo do método da separação de variáveis que nos permite escolher soluções do tipo $\Psi(\mathbf{r}, t)=X(x, t) Y(y, t) Z(z, t)$ e definindo $\Psi(z, t) \equiv Z(z, t)$. Devido a esta escolha, o operador de energia cinética passa a ser somente

$$
\mathbf{T}=-\frac{\hbar^{2}}{2 m} \frac{\partial^{2}}{\partial z^{2}}
$$

A ligação entre $\Psi(z, t)$ e a probabilidade por unidade de comprimento ou densidade de probabilidade $P(z, t)$ foi proposta por Born (1926) através do postulado:[10]

Se é feita, no instante $t$, uma medida para se localizar a partícula associada à função de onda $\Psi(z, t)$, então a probabilidade $P(z, t)$ de que a partícula seja encontrada entre $z$ e $z+d z$ é dada por:

$$
P(z, t) d z=\Psi^{*}(z, t) \Psi(z, t)
$$

onde o símbolo $\Psi^{*}(z, t)$ representa a função complexo conjugado de $\Psi(z, t)$. É claro que devemos ter

$$
\int_{-\infty}^{+\infty} \Psi^{*}(z, t) \Psi(z, t) d z=1
$$

uma vez que esta integral representa a probabilidade total de que a partícula associada à função de onda $\Psi(z, t)$ esteja em algum lugar do eixo dos $z$, no instante $t$, para todos os valores de $t$. Em outras palavras, as funções de onda devem ser normalizadas.

Para uma partícula movimentando-se na presença de um potencial independente do tempo, $\mathbf{V}=\mathbf{V}(z)$, uma técnica comum para a solução da equação de Schrödinger (2.14) consiste em procurar soluções da forma

$$
\Psi(z, t)=\psi(z) \phi(t)
$$

que são um produto de uma função $\psi(z)$ que depende só da posição $z$ por uma função $\phi(t)$ que depende só do tempo $t$. Substituindo a relação (2.19) em (2.14) resulta:

$$
i \hbar \psi(z) \frac{\partial}{\partial t} \phi(t)=-\frac{\hbar^{2}}{2 m} \phi(t) \frac{\partial^{2}}{\partial z^{2}} \psi(z)+V(z) \psi(z) \phi(t)
$$


Dividindo a expressão (2.20) por $\Psi(z, t)$ obtemos uma equação cujo lado esquerdo depende apenas do tempo e o lado direito depende apenas da coordenada espacial:

$$
i \hbar \frac{1}{\phi(t)} \frac{\partial}{\partial t} \phi(t)=-\frac{\hbar^{2}}{2 m} \frac{1}{\psi(z)} \frac{\partial^{2}}{\partial z^{2}} \psi(z)+V(z) .
$$

Essa igualdade só pode ser verdadeira se ambos os lados forem constantes. Assim, é possível separar a Eq. (2.21) nas Eqs. (2.22) e (2.23), que são acopladas através da constante E:

$$
\begin{gathered}
\frac{\partial}{\partial t} \phi(t)=-\frac{i E}{\hbar} \phi(t) \\
-\frac{\hbar^{2}}{2 m} \frac{\partial^{2}}{\partial z^{2}} \psi(z)+V(z) \psi(z)=E \psi(z) .
\end{gathered}
$$

Embora a Eq. (2.22) tenha uma solução simples para todo $E$,

$$
\phi(t)=A \exp \left(-\frac{i}{\hbar} E t\right)
$$

a solução da Eq. (2.23), conhecida como equação de Schrödinger independente do tempo (ESIT), dependerá da forma do potencial.

Como a Eq. (2.23) é linear, se $\psi_{1}(z)$ e $\psi_{2}(z)$ forem soluções, qualquer combinação linear destas duas funções também será uma solução válida. Em particular, é possível encontrar uma base de funções ortogonais $\left\{\psi_{\mathrm{n}}(z)\right\}$ que sejam soluções da Eq. (2.23) para um conjunto de constantes $\left\{E_{\mathrm{n}}\right\}$, de forma que qualquer solução $\psi(z)$ pode ser escrita como:

$$
\psi(z)=\sum_{\mathrm{n}} c_{\mathrm{n}} \psi_{\mathrm{n}}(z)
$$

onde $c_{\mathrm{n}}=\int_{-\infty}^{\infty} \psi_{\mathrm{n}}^{*}(z) \psi(z) d z$ são constantes complexas. As funções $\psi_{\mathrm{n}}(z)$ e os valores $E_{\mathrm{n}}$ são chamados autofunções e autoenergias do hamiltoniano $\mathbf{H}$, respectivamente.

Se um sistema for caracterizado por uma autofunção $\psi_{\mathrm{n}}(z)$, podemos escrever a solução da equação de Schrödinger (Eq. (2.14)) que descreve a evolução deste sistema como:

$$
\psi_{\mathrm{n}}(z, t)=\psi_{\mathrm{n}}(z) e^{-\frac{i}{\hbar} E_{\mathrm{n}} t}
$$

Estados como os descritos em (2.26) são chamados estacionários, pois a densidade de probabilidade de posição, $|\Psi(z, t)|^{2}$, é independente do tempo:

$$
\left|\psi_{\mathrm{n}}(z, t)\right|^{2}=\left(\psi_{\mathrm{n}}(z) e^{-\frac{i}{\hbar} E_{\mathrm{n}} t}\right)^{*}\left(\psi_{\mathrm{n}}(z) e^{-\frac{i}{\hbar} E_{\mathrm{n}} t}\right)=\left|\psi_{\mathrm{n}}(z)\right|^{2}
$$

pois $\left|e^{-\frac{i}{\hbar} E_{\mathrm{n}} t}\right|^{2}=1$. Observe que o estado descrito pela função de onda dada em (2.26) é apenas uma solução particular da Eq. (2.14). De fato, é possível encontrar um conjunto completo e ortonormal de funções $\left\{\psi_{\mathrm{n}}(z)\right\}$ que são soluções de (2.23) para as constantes $\left\{E_{\mathrm{n}}\right\}$. 
A solução geral para a ESDT, Eq. (2.14), quando o potencial não depende do tempo, pode ser expressa como:

$$
\Psi(z, t)=\sum_{\mathrm{n}} c_{\mathrm{n}} \psi_{\mathrm{n}}(z) e^{-\frac{i}{\hbar} E_{\mathrm{n}} t}
$$

Aqui vale ressaltar a total equivalência entre as equações (2.11) e (2.28). A primeira foi obtida apenas trabalhando a ação do operador de evolução temporal sobre um estado qualquer do espaço de Hilbert de $\mathbf{H}$ projetado na representação das coordenadas, enquanto que a segunda foi obtida resolvendo a ESDT para a função de onda do estado já projetado na representação das coordenadas desde o início. Esta equivalência entre a solução da ESDT e a ação de um operador de evolução será explorada bastante frequentemente no decorrer dos próximos capítulos.

Como vemos, a evolução de um sistema quântico para um hamiltoniano que não depende do tempo é feita, normalmente, resolvendo-se a $\operatorname{ESIT~} \mathbf{H} \psi(z)=E \psi(z)$ onde procuramos os pares $\left(\left\{E_{\mathrm{n}}\right\},\left\{\psi_{\mathrm{n}}(z)\right\}\right)$ que descrevem estados estacionários do sistema. Encontrar os estados estacionários de um sistema quântico não é tarefa elementar e constitui problema central na Mecânica Quântica. Exceto para alguns potenciais com formas específicas, que têm soluções analíticas, para a grande maioria dos potenciais de interesse deve-se utilizar métodos numéricos para a determinação das autoenergias e dos autoestados da ESIT. A maneira mais comum de abordar este problema numérico seria diagonalizar diretamente o hamiltoniano ao selecionar um número relevante de funções necessárias para expandir com precisão as soluções. No entanto, as técnicas de diagonalização direta são muito exigentes computacionalmente, especialmente para sistemas grandes, ou com geometrias complexas. Uma das alternativas é desenvolver abordagens numéricas para resolver a equação de Schrödinger diretamente, sem a expansão em estados estacionários.

É importante notar também que quando $\mathbf{V}(z, t)$ é dependente do tempo, as equações (2.11) e (2.28) não são mais válidas. No Capítulo 4 descrevemos alguns métodos numéricos que nos permitem encontrar as autofunções e autoenergias de $\mathbf{H}$ para qualquer potencial, mesmo que dependente do tempo.

\subsection{Propriedades}

Nesta seção apresentaremos a formulação utilizada para o cálculo da fotocorrente induzida por um campo elétrico variável no tempo em heteroestruturas semicondutoras e também a teoria usada no cálculo do espectro de absorção de fótons por elétrons confinados nos estados ligados nestas hereroestruturas.[6, 11] 


\subsubsection{Fotocorrente}

Por fotocorrente, nos referimos à corrente elétrica que pode surgir em um dispositivo em razão da incidência de fótons. Por exemplo, elétrons confinados em poços quânticos podem gerar corrente elétrica caso absorvam fótons com energia suficientemente grande para ultrapassarem as barreiras, como exemplificado na Figura 1. Note que uma diferença de potencial tem o papel de curvar a banda de condução, fornecendo um viés para a direção na qual o elétron irá se deslocar.[6]

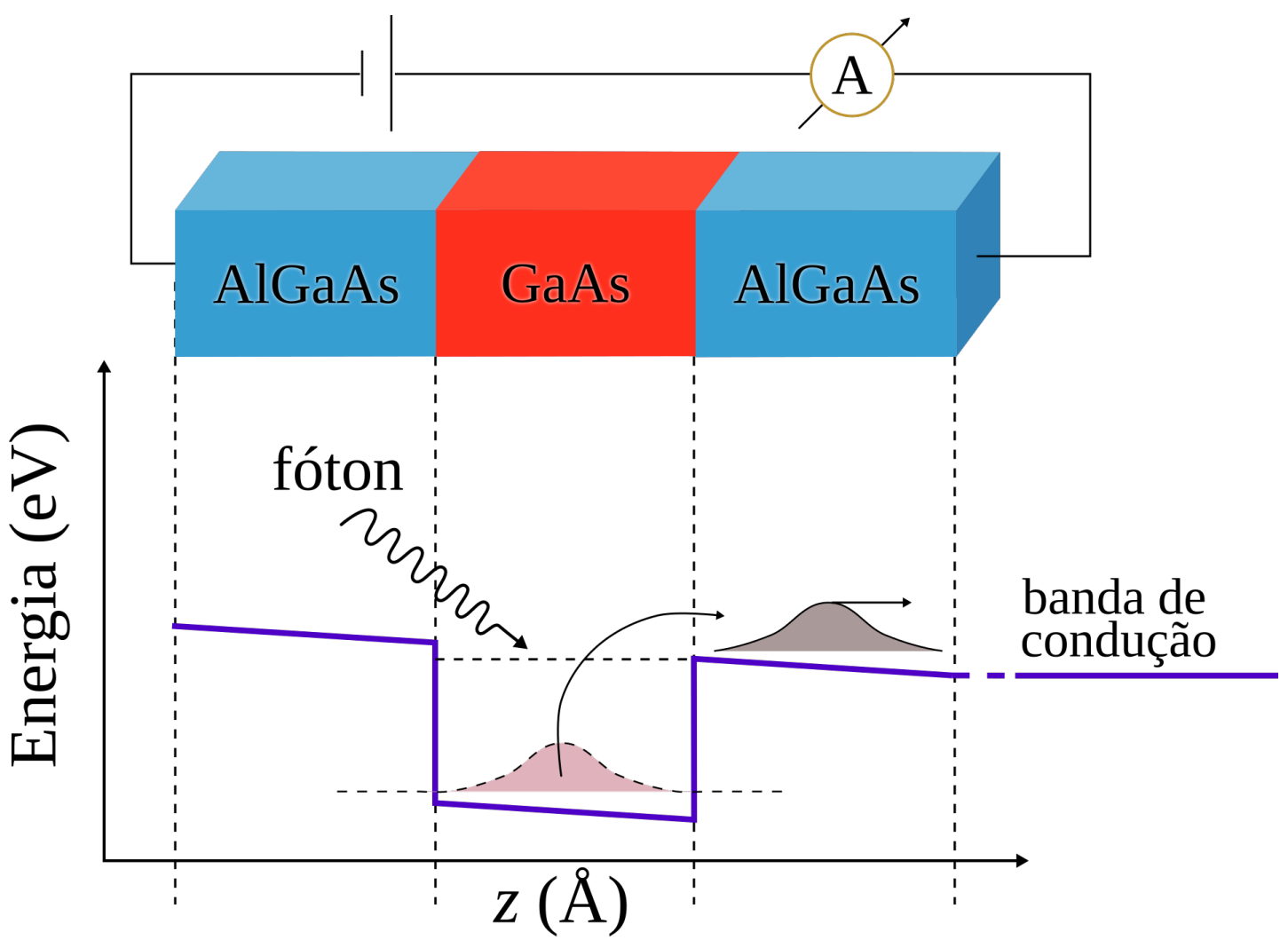

Figura 1: Ideia simplificada de como a absorção de um fóton excita um elétron confinado em um poço quântico no nível $n_{1}$ para um estado de condução no contínuo.

O ponto de partida para o cálculo desta fotocorrente é a equação da conservação de carga do eletromagnetismo

$$
\frac{\partial \rho}{\partial t}=-\vec{\nabla} \cdot \mathbf{J}
$$

onde $\rho$ é a densidade de carga e $\mathbf{J}$ é a densidade de corrente.

Se um elétron estiver no estado (normalizado) $\Psi(\mathbf{r}, t)$, a probabilidade de encontrálo em um volume $d^{3} \mathbf{r}$ ao redor do ponto $\mathbf{r}$ é dada por $|\Psi(\mathbf{r}, t)|^{2} d^{3} \mathbf{r}$. Sendo $|\Psi(\mathbf{r}, t)|^{2}$ a densidade de probabilidade espacial, então a densidade de carga é $\rho=-e|\Psi(\mathbf{r}, t)|^{2}$, onde $e$ é a magnitude da carga elétrica elementar. De fato, para um elétron de carga $-e$ devemos ter

$$
-e=\int d^{3} \mathbf{r} \rho(\mathbf{r})=\int d^{3} \mathbf{r}\left(-e|\Psi(\mathbf{r}, t)|^{2}\right)=-e \int d^{3} \mathbf{r}|\Psi(\mathbf{r}, t)|^{2}=-e
$$


Utilizando a E. (2.29) juntamente com a ESDT, Eq. (2.14), vemos que

$$
\begin{aligned}
\frac{\partial \rho}{\partial t} & =-e \frac{\partial|\Psi(\mathbf{r}, t)|^{2}}{\partial t}=-e \frac{\partial \Psi^{*}(\mathbf{r}, t) \Psi(\mathbf{r}, t)}{\partial t} \\
& =-e\left(\Psi(\mathbf{r}, t) \frac{\partial \Psi^{*}(\mathbf{r}, t)}{\partial t}+\Psi^{*}(\mathbf{r}, t) \frac{\partial \Psi(\mathbf{r}, t)}{\partial t}\right) \\
& =-e\left[\Psi(\mathbf{r}, t)\left(-\frac{1}{i \hbar} \mathbf{H} \Psi^{*}(\mathbf{r}, t)\right)-\Psi^{*}(\mathbf{r}, t)\left(\frac{1}{i \hbar} \mathbf{H} \Psi(\mathbf{r}, t)\right)\right] \\
& =\frac{e}{i \hbar}\left[\Psi(\mathbf{r}, t)(\mathbf{T}+\mathbf{V}(\mathbf{r}, t)) \Psi^{*}(\mathbf{r}, t)-\Psi^{*}(\mathbf{r}, t)(\mathbf{T}+\mathbf{V}(\mathbf{r}, t)) \Psi(\mathbf{r}, t)\right] .
\end{aligned}
$$

Como estamos na representação das coordenadas tanto $\Psi(\mathbf{r}, t)$ quanto $\Psi^{*}(\mathbf{r}, t)$ comutam com $\mathbf{V}(\mathbf{r}, t)$, portanto

$$
\Psi(\mathbf{r}, t) \mathbf{V}(\mathbf{r}, t) \Psi^{*}(\mathbf{r}, t)-\Psi^{*}(\mathbf{r}, t) \mathbf{V}(\mathbf{r}, t) \Psi(\mathbf{r}, t)=0 .
$$

Utilizando o operador de energia cinética para uma partícula de massa efetiva com dependência espacial, Eq. (3.9), temos

$$
\begin{aligned}
\frac{\partial \rho}{\partial t}= & -\frac{e \hbar}{2 i}\left[\Psi(\mathbf{r}, t)\left(\vec{\nabla}\left(\frac{1}{m^{*}(\mathbf{r})} \vec{\nabla}\right)\right) \Psi^{*}(\mathbf{r}, t)-\Psi^{*}(\mathbf{r}, t)\left(\vec{\nabla}\left(\frac{1}{m^{*}(\mathbf{r})} \vec{\nabla}\right)\right) \Psi(\mathbf{r}, t)\right] \\
= & -\frac{e \hbar}{2 i}\left[\Psi(\mathbf{r}, t)\left(\vec{\nabla}\left(\frac{1}{m^{*}(\mathbf{r})}\right) \vec{\nabla} \Psi^{*}(\mathbf{r}, t)+\frac{1}{m^{*}(\mathbf{r})} \vec{\nabla}^{2} \Psi^{*}(\mathbf{r}, t)\right)\right] \\
& +\frac{e \hbar}{2 i}\left[\Psi^{*}(\mathbf{r}, t)\left(\vec{\nabla}\left(\frac{1}{m^{*}(\mathbf{r})}\right) \vec{\nabla} \Psi(\mathbf{r}, t)+\frac{1}{m^{*}(\mathbf{r})} \vec{\nabla}^{2} \Psi(\mathbf{r}, t)\right)\right] \\
= & -\vec{\nabla} \cdot\left[\frac{e \hbar}{2 i m^{*}(\mathbf{r})}\left(\Psi(\mathbf{r}, t) \vec{\nabla} \Psi^{*}(\mathbf{r}, t)-\Psi^{*}(\mathbf{r}, t) \vec{\nabla} \Psi(\mathbf{r}, t)\right)\right]=-\vec{\nabla} \cdot \mathbf{J},
\end{aligned}
$$

e podemos identificar

$$
\mathbf{J}(\mathbf{r}, t)=\frac{e \hbar}{2 i m^{*}(\mathbf{r})}\left[\Psi(\mathbf{r}, t) \vec{\nabla} \Psi^{*}(\mathbf{r}, t)-\Psi^{*}(\mathbf{r}, t) \vec{\nabla} \Psi(\mathbf{r}, t)\right] .
$$

A Eq. (2.34) é desenvolvida nos textos de Mecânica Quântica para uma partícula de massa $m$ invariante no espaço.[8, 11, 12] Aqui nós mostramos que ela é valida mesmo para um massa que varia no espaço e que esta dependência não modifica a estrutura da expressão; tomamos apenas a liberdade de não fazer $e=1 .[12]$

Como estamos interessados em sistemas unidimensionais, iremos particularizar o problema para uma dimensão. Assim sendo, se conhecermos o estado $\Psi(z, t)$ ao longo de um intervalo de tempo $T$ podemos estimar a fotocorrente $I$ entre dois pontos $z_{1}$ e $z_{2}$ como sendo $[6]$

$$
I=\frac{1}{T} \int_{0}^{T} d t\left[J\left(z_{2}, t\right)-J\left(z_{1}, t\right)\right]
$$

\subsubsection{Absorção Intersubbanda}

Para o cálculo do coeficiente de absorção em heteroestruturas semicondutoras, mais especificamente em poços quânticos, devemos descrever a propagação de uma onda 
eletromagnética no material semicondutor e distinguir quais são as formas que um elétron no semicondutor pode absorver um fóton da onda eletromagnética. As transições fotônicas possíveis estão representadas na Figura 2:

1. Transições interbandas que ocorrem entre a banda de condução e a banda de valência e envolvem dois tipos de portadores (eles são bipolares), elétrons e buracos. Neste caso, a energia da transição é a energia do gap mais as energias de confinamento dos elétrons e buracos menos a energia de ligação do éxciton.

2. Transições intrabandas que acontecem dentro da banda de condução ou de valência e envolvem apenas um tipo de portador (a transição é unipolar). Nos poços quânticos existem subbandas dentro da banda de condução ou da banda de valência. Transições intrabandas nessas estruturas entre duas subbandas são chamadas transições intersubbandas. Observe que outro tipo de transição intrabanda pode ocorrer que envolve as transições de um portador de uma subbanda para a mesma subbanda com absorção de um fóton e emissão de um fônon (conservação de momento).

Nesta subseção apresentamos o formalismo para o cálculo do coeficiente de absorção intersubbanda em poços quânticos. Para obter a equação Schrödinger para uma partícula carregada em um campo eletromagnético externo, primeiro examinaremos a hamiltoniana clássica de uma partícula carregada em um campo eletromagnético. Em seguida, apresentamos o formalismo baseado em dinâmica quântica que nos permite calcular o coeficiente de absorção intersubbanda.

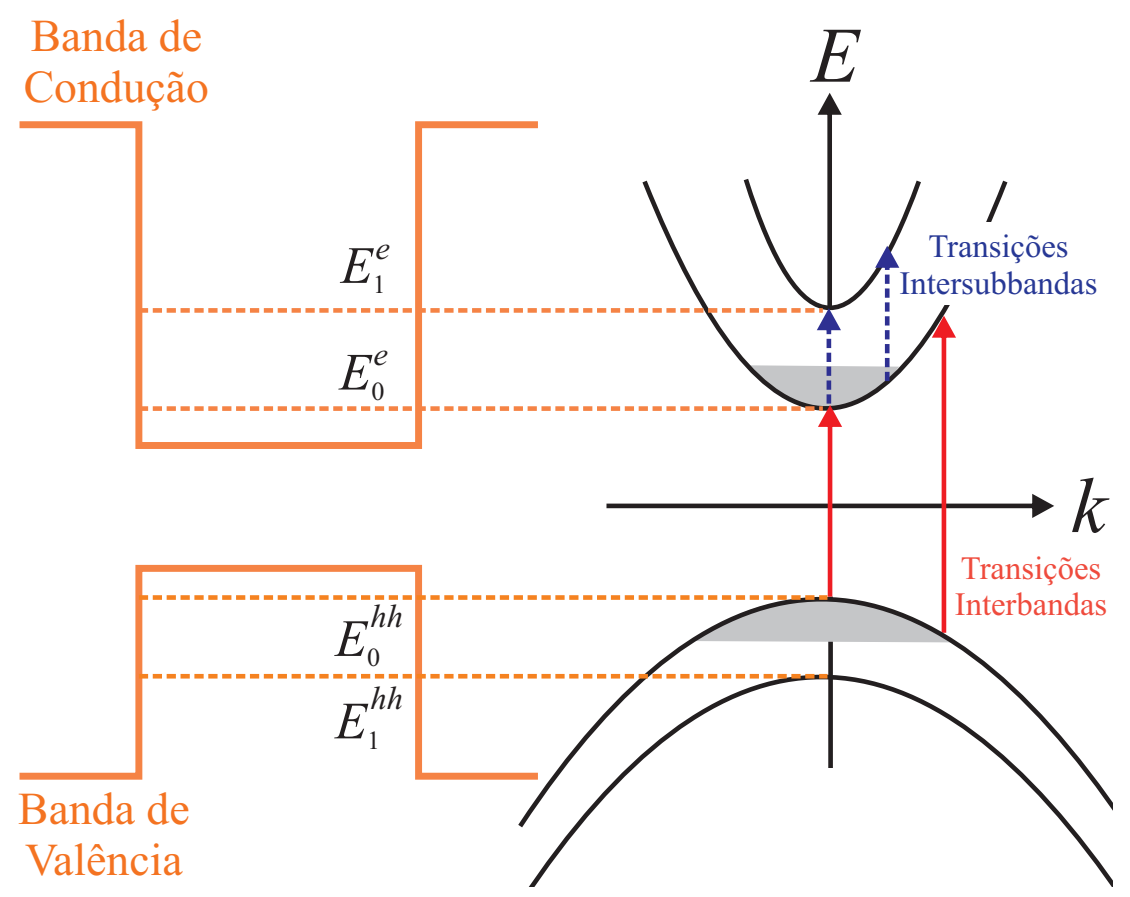

Figura 2: Representação das transições interbandas e intrasubbandas em poços quânticos. $E_{i}^{e}\left(E_{i}^{h h}\right)$ indicam as energias dos elétrons (buracos) confinados no poço quântico. 


\subsubsection{Interação Radiação-Matéria}

A hamiltoniana clássica de uma partícula de carga $q$ e massa $m$ na presença de campos elétrico $\overrightarrow{\mathbf{E}}(\overrightarrow{\mathbf{r}}, t)$ e magnético $\overrightarrow{\mathbf{B}}(\overrightarrow{\mathbf{r}}, t)$ externos e de um potencial externo $V(\overrightarrow{\mathbf{r}})$, que não é relacionado com o campo eletromagnético externo, é dada por:[13]

$$
\mathbf{H}=\frac{1}{2 m}\left[\overrightarrow{\mathbf{p}}-\frac{q \overrightarrow{\mathbf{A}}(\overrightarrow{\mathbf{r}}, t)}{c}\right]^{2}+q \Phi+V(\overrightarrow{\mathbf{r}})
$$

onde o potencial elétrico $\Phi(\overrightarrow{\mathbf{r}}, t)$ e o potencial vetor $\vec{A}(\overrightarrow{\mathbf{r}}, t)$ estão relacionados com os campos elétrico e magnético pelas relações:

$$
\begin{gathered}
\overrightarrow{\mathbf{E}}(\overrightarrow{\mathbf{r}}, t)=-\overrightarrow{\tilde{\nabla}} \Phi(\overrightarrow{\mathbf{r}}, t)-\frac{1}{c} \frac{\partial \overrightarrow{\mathbf{A}}(\overrightarrow{\mathbf{r}}, t)}{\partial t} \quad(\mathrm{CGS}) \\
\overrightarrow{\mathbf{B}}(\overrightarrow{\mathbf{r}}, t)=\overrightarrow{\tilde{\nabla}} \times \overrightarrow{\mathbf{A}}(\overrightarrow{\mathbf{r}}, t) \quad(\mathrm{CGS})
\end{gathered}
$$

Na representação das coordenadas, identificamos o momento $\overrightarrow{\mathbf{p}}$ com o operador diferencial $-i \hbar \vec{\nabla}$. Como estamos interessados nas transições na banda de condução, temos que utilizar $q=-e$ onde $e$ é a magnitude da carga elementar. Desta forma, a equação de Schrödinger será dada por:

$$
\frac{1}{2 m}\left[-i \hbar \vec{\nabla}+\frac{e \overrightarrow{\mathbf{A}}(\overrightarrow{\mathbf{r}}, t)}{c}\right]^{2} \Psi(\overrightarrow{\mathbf{r}}, t)-e \Phi(\overrightarrow{\mathbf{r}}, t) \Psi(\overrightarrow{\mathbf{r}}, t)+V(\overrightarrow{\mathbf{r}}) \Psi(\overrightarrow{\mathbf{r}}, t)=i \hbar \frac{\partial \Psi(\overrightarrow{\mathbf{r}}, t)}{\partial t}
$$

A expressão (2.39) pode ser expandida como:

$$
-\frac{\hbar^{2}}{2 m} \vec{\nabla}^{2} \Psi-\frac{i e \hbar}{2 m c} \overrightarrow{\mathbf{A}} \cdot \vec{\nabla} \Psi-\frac{i e \hbar}{2 m c} \vec{\nabla} \cdot(\overrightarrow{\mathbf{A}} \Psi)+\frac{e^{2}}{2 m c^{2}} \overrightarrow{\mathbf{A}}^{2} \Psi-e \Phi \Psi+V \Psi=i \hbar \frac{\partial \Psi}{\partial t}
$$

onde omitimos os argumentos nas coordenadas $(\overrightarrow{\mathbf{r}}, t)$, nos potenciais e na função de onda $\Psi$. Podemos simplificar a expressão anterior escolhendo o gauge de $\operatorname{Coulomb}(\vec{\nabla} \cdot \overrightarrow{\mathbf{A}}=0$ e $\Phi=0)$, que leva à igualdade $\vec{\nabla} \cdot(\overrightarrow{\mathbf{A}} \Psi)=\Psi(\vec{\nabla} \cdot \overrightarrow{\mathbf{A}})+\overrightarrow{\mathbf{A}} \cdot \vec{\nabla} \Psi=\overrightarrow{\mathbf{A}} \cdot \vec{\nabla} \Psi$. Também desconsideramos termos não lineares em $\overrightarrow{\mathbf{A}}$, o que se justifica para campos com baixa intensidade, de modo que a equação de Schrödinger reduz-se à:

$$
\left[-\frac{\hbar^{2}}{2 m} \vec{\nabla}^{2}+V\right] \Psi-\frac{i e \hbar}{m c} \overrightarrow{\mathbf{A}} \cdot \vec{\nabla} \Psi=i \hbar \frac{\partial \Psi}{\partial t} .
$$

Vamos assumir que o campo elétrico da onda eletromagnética, que se propaga como onda plana no material com índice de refração $n$, caracterizado pela frequência angular $\omega$ e pelo vetor de onda $\overrightarrow{\mathbf{k}}\left(|\overrightarrow{\mathbf{k}}|=\frac{n \omega}{c}\right)$, seja escrito como

$$
\overrightarrow{\mathbf{E}}(\overrightarrow{\mathbf{r}}, t)=\mathrm{E} \vec{\epsilon} \cos (\omega t-\overrightarrow{\mathbf{k}} \cdot \overrightarrow{\mathbf{r}})
$$

sendo $\vec{\epsilon}$ o vetor polarização (suposto linear), que é perpendicular ao vetor $\overrightarrow{\mathbf{k}}$. Com esta escolha, o potencial vetor que satisfaz a Eq. (2.37) é dado por:

$$
\overrightarrow{\mathbf{A}}(\overrightarrow{\mathbf{r}}, t)=-\frac{\vec{\epsilon} c \mathrm{E}}{2 i \omega}\{\exp [i(\omega t-\overrightarrow{\mathbf{k}} \cdot \overrightarrow{\mathbf{r}})]-\exp [-i(\omega t-\overrightarrow{\mathbf{k}} \cdot \overrightarrow{\mathbf{r}})]\}
$$


O comprimento de onda da radiação infravermelha que nos interessa (no intervalo de $0,8 \mu \mathrm{m}$ e $1000 \mu \mathrm{m}$ ) é bem maior que a extensão das autofunções nos poços quânticos (que têm largura da ordem de dezenas de angstrons). Assim, pode considerar-se que o campo elétrico é constante no espaço, aproximação esta conhecida como aproximação de dipolo elétrico. Isso significa adotar $\overrightarrow{\mathbf{k}}=0$. Com isso o potencial vetor será dado por:

$$
\overrightarrow{\mathbf{A}}(\overrightarrow{\mathbf{r}}, t)=-\frac{\vec{\epsilon} c \mathrm{E}}{2 i \omega}\{\exp [i \omega t]-\exp [-i \omega t]\}
$$

Substituindo a expressão do potencial vetor na equação de Schrödinger (Eq. 2.41) resulta:

onde

$$
\mathbf{H}_{0} \Psi+\mathbf{V}_{\mathrm{EM}}(t) \Psi=i \hbar \frac{\partial \Psi}{\partial t}
$$

$$
\mathbf{H}_{0}=\left[-\frac{\hbar^{2}}{2 m} \vec{\nabla}^{2}+V\right]
$$

e

$$
\mathbf{V}_{\mathrm{EM}}(t)=-\frac{i e \hbar}{m c} \overrightarrow{\mathbf{A}} \cdot \vec{\nabla}=\frac{e}{m c} \overrightarrow{\mathbf{A}} \cdot \overrightarrow{\mathbf{p}}=-\frac{e E}{2 i m \omega}\left\{e^{i \omega t}-e^{-i \omega t}\right\} \vec{\epsilon} \cdot \overrightarrow{\mathbf{p}}
$$

Podemos escrever operador hamiltoniano da Eq. (2.46) como:

$$
\mathbf{H}_{0}=\frac{\overrightarrow{\mathbf{p}}^{2}}{2 m}+V(\overrightarrow{\mathbf{r}})
$$

de modo que o comutador: [8]

$$
\left[\overrightarrow{\mathbf{r}}, \mathbf{H}_{0}\right]=\frac{1}{2 m}\left[\overrightarrow{\mathbf{r}}, \overrightarrow{\mathbf{p}}^{2}\right]=\frac{1}{2 m}(\overrightarrow{\mathbf{p}}[\overrightarrow{\mathbf{r}}, \overrightarrow{\mathbf{p}}]+[\overrightarrow{\mathbf{r}}, \overrightarrow{\mathbf{p}}] \overrightarrow{\mathbf{p}})=\frac{i \hbar}{m} \overrightarrow{\mathbf{p}}
$$

pois $[\overrightarrow{\mathbf{r}}, \overrightarrow{\mathbf{p}}]=i \hbar$. Portanto, o momento linear pode ser escrito como:

$$
\overrightarrow{\mathbf{p}}=-\frac{i m}{\hbar}\left[\overrightarrow{\mathbf{r}}, \mathbf{H}_{0}\right]=-\frac{i m}{\hbar}\left(\overrightarrow{\mathbf{r}} \mathbf{H}_{0}-\mathbf{H}_{0} \overrightarrow{\mathbf{r}}\right) \text {. }
$$

Isso significa que qualquer elemento de matriz de $\mathbf{V}_{\mathrm{EM}}(t)$ na base dos autovetores de $\mathbf{H}_{0}$ será da forma:

$$
\begin{aligned}
\left\langle n\left|\mathbf{V}_{\mathrm{EM}}(t)\right| m\right\rangle & \propto \int d^{3} \overrightarrow{\mathbf{r}} \psi_{n}(\overrightarrow{\mathbf{r}})\left(\overrightarrow{\mathbf{r}} \mathbf{H}_{0}-\mathbf{H}_{0} \overrightarrow{\mathbf{r}}\right) \psi_{m}(\overrightarrow{\mathbf{r}}) \\
& \propto \int d^{3} \overrightarrow{\mathbf{r}} \psi_{n}(\overrightarrow{\mathbf{r}})\left(\overrightarrow{\mathbf{r}} \mathbf{H}_{0}\right) \psi_{m}(\overrightarrow{\mathbf{r}})-\psi_{n}(\overrightarrow{\mathbf{r}})\left(\mathbf{H}_{0} \overrightarrow{\mathbf{r}}\right) \psi_{m}(\overrightarrow{\mathbf{r}}) \\
& \propto \int d^{3} \overrightarrow{\mathbf{r}} \psi_{n}(\overrightarrow{\mathbf{r}})\left(\overrightarrow{\mathbf{r}} E_{\mathrm{m}}\right) \psi_{m}(\overrightarrow{\mathbf{r}})-\psi_{n}(\overrightarrow{\mathbf{r}})\left(E_{\mathrm{n}} \overrightarrow{\mathbf{r}}\right) \psi_{m}(\overrightarrow{\mathbf{r}}) \\
& \propto\left(E_{\mathrm{m}}-E_{\mathrm{n}}\right) \int d^{3} \overrightarrow{\mathbf{r}} \psi_{n}(\overrightarrow{\mathbf{r}})(\overrightarrow{\mathbf{r}}) \psi_{m}(\overrightarrow{\mathbf{r}}) \\
& \propto\left(E_{\mathrm{m}}-E_{\mathrm{n}}\right)\langle n|\overrightarrow{\mathbf{r}}| m\rangle .
\end{aligned}
$$

A Eq. (2.51) nos permite reescrever $\mathbf{V}_{\mathrm{EM}}(t)$ como:

$$
\begin{aligned}
\mathbf{V}_{\mathrm{EM}}(t) & =-\frac{e E}{2 i m \omega}\left\{e^{i \omega t}-e^{-i \omega t}\right\} \vec{\epsilon} \cdot(i m \omega \overrightarrow{\mathbf{r}}) \\
& =-e E \cos (\omega t) \vec{\epsilon} \cdot \overrightarrow{\mathbf{r}}=-e \overrightarrow{\mathbf{E}}(t) \cdot \overrightarrow{\mathbf{r}},
\end{aligned}
$$

onde chamamos $\omega=-\left(E_{\mathrm{n}}-E_{\mathrm{m}}\right) / \hbar$, pois são as frequências onde ocorrem absorção.[9] 


\subsubsection{Espectro de Absorção}

O espectro de absorção $\sigma(\omega)$ é a razão entre o número de fótons absorvidos $-\Delta N(\omega)^{2}$ e número de fótons incidentes $N(\omega)$ por unidade de área $A$ para uma dada frequência $\omega .[9]$ Um campo eletromagnético oscilante atuando na região de uma heteroestrutura semicondutora é uma boa forma de representar a incidência destes fótons sobre o material.

É possível considerar a atuação apenas do campo elétrico, desprezando o campo magnético ${ }^{3}$. O hamiltoniano total do sistema, utilizando as equações (2.46) e (2.52) fica então:

$$
\begin{aligned}
\mathbf{H} & =\mathbf{H}_{0}-e \overrightarrow{\mathbf{E}}(t) \cdot \overrightarrow{\mathbf{r}} \\
& =\mathbf{H}_{0}-\mathbf{E}(t) \cdot \boldsymbol{\mu} .
\end{aligned}
$$

sendo $\mathbf{H}_{0}$ um sistema com solução conhecida $\left(\mathbf{H}_{0}|\mathrm{n}\rangle=E_{\mathrm{n}}|\mathrm{n}\rangle\right)$, e a carga elétrica elementar, $\mathbf{r}$ a coordenada espacial e $\overrightarrow{\mathbf{E}}(t)$ o campo elétrico oscilante. Esta escolha recebe o nome de aproximação de dipolo elétrico, sendo $\overrightarrow{\boldsymbol{\mu}}=e \overrightarrow{\mathbf{r}}$ o momento de dipolo elétrico de uma única carga e. $[6,9,12]$

Podemos ainda, escrever o hamiltoniano da Eq. (2.53) utilizando a representação diagonal: $[9,14]$

$$
\begin{aligned}
\mathbf{H} & =\sum_{\mathrm{n}} E_{\mathrm{n}}|\mathrm{n}\rangle\langle\mathrm{n}|-\mathbf{E}(t) \cdot \mathbb{1} \boldsymbol{\mu} \mathbb{1} \\
& =\sum_{\mathrm{n}} E_{\mathrm{n}}|\mathrm{n}\rangle\left\langle\mathrm{n}\left|-\mathbf{E}(t) \cdot \sum_{\mathrm{n}, \mathrm{m}}\right| \mathrm{n}\right\rangle\langle\mathrm{n}|\boldsymbol{\mu}| \mathrm{m}\rangle\langle\mathrm{m}| \\
& =\sum_{\mathrm{n}} E_{\mathrm{n}}|\mathrm{n}\rangle\left\langle\mathrm{n}\left|-\mathbf{E}(t) \cdot \sum_{\mathrm{n}, \mathrm{m}} \boldsymbol{\mu}_{\mathrm{n}, \mathrm{m}}\right| \mathrm{n}\right\rangle\langle\mathrm{m}|
\end{aligned}
$$

onde $\boldsymbol{\mu}_{\mathrm{n} . \mathrm{m}}=\langle\mathrm{n}|\mu| \mathrm{m}\rangle$.

Definimos a população de um nível $|\mathrm{a}\rangle$ como sendo $N_{\mathrm{a}}=\langle\mathrm{a} \mid \mathrm{a}\rangle$. De modo que a taxa de variação temporal desta população é dada por:

$$
\frac{d N_{\mathrm{a}}}{d t}=\frac{\partial\langle\mathrm{a} \mid \mathrm{a}\rangle}{\partial t}=\frac{\partial\langle\mathrm{a}|}{\partial t}|\mathrm{a}\rangle+\langle\mathrm{a}| \frac{\partial|\mathrm{a}\rangle}{\partial t}
$$

onde $|\mathrm{a}\rangle=|\mathrm{a} ; t\rangle$, mas vamos deixar a dependência temporal implícita para facilitar a compreensão. Utilizamos agora a própria equação de Schrödinger

$$
\begin{aligned}
\frac{\partial|\mathrm{a}\rangle}{\partial t} & =-\frac{i}{\hbar} \mathbf{H}|\mathrm{a}\rangle \\
\frac{\partial\langle\mathrm{a}|}{\partial t} & =\langle\mathrm{a}| \frac{i}{\hbar} \mathbf{H}^{\dagger}
\end{aligned}
$$

2 O número $\Delta N$ é na verdade o saldo líquido de elétrons atingindo ou deixando um determinado nível. Um saldo negativo indica que elétrons foram excitados para outros níveis, ou seja, $-\Delta N$ é o número de fótons absorvidos.

3 Para problemas ópticos, esta escolha facilita a modelagem e não apresenta diferenças em relação a Eq. (2.41).[12] 
juntamente com a Eq. (2.54) para escrever:

$$
\begin{aligned}
\frac{d N_{\mathrm{a}}}{d t}= & {\left[\langle\mathrm{a}| \frac{i}{\hbar} \mathbf{H}^{\dagger}\right]|\mathrm{a}\rangle+\langle\mathrm{a}|\left[-\frac{i}{\hbar} \mathbf{H}|\mathrm{a}\rangle\right] } \\
= & \frac{i}{\hbar}\left\langle\mathrm{a}\left|\left[\sum_{\mathrm{n}} E_{\mathrm{n}}|\mathrm{n}\rangle\left\langle\mathrm{n}\left|-\mathbf{E}^{*}(t) \cdot \sum_{\mathrm{n}, \mathrm{m}} \boldsymbol{\mu}_{\mathrm{m}, \mathrm{n}}\right| \mathrm{m}\right\rangle\langle\mathrm{n}|\right]\right| \mathrm{a}\right\rangle \\
& -\frac{i}{\hbar}\left\langle\mathrm{a}\left|\left[\sum_{\mathrm{n}} E_{\mathrm{n}}|\mathrm{n}\rangle\left\langle\mathrm{n}\left|-\mathbf{E}(t) \cdot \sum_{\mathrm{n}, \mathrm{m}} \boldsymbol{\mu}_{\mathrm{n}, \mathrm{m}}\right| \mathrm{n}\right\rangle\langle\mathrm{m}|\right]\right| \mathrm{a}\right\rangle \\
= & \frac{i}{\hbar}\left\langle\mathrm { a } \left|\left[E_{\mathrm{a}}|\mathrm{a}\rangle-\mathbf{E}^{*}(t) \cdot \sum_{\mathrm{m}} \boldsymbol{\mu}_{\mathrm{m}, \mathrm{a}}|\mathrm{m}\rangle\right]-\frac{i}{\hbar}\left\langle\mathrm { a } \left|\left[E_{\mathrm{a}}|\mathrm{a}\rangle-\mathbf{E}(t) \cdot \sum_{\mathrm{n}} \boldsymbol{\mu}_{\mathrm{n}, \mathrm{a}}|\mathrm{n}\rangle\right]\right.\right.\right.\right. \\
= & \frac{i}{\hbar} \mathbf{E}^{*}(t) \cdot \sum_{\mathrm{m}}\left\langle\mathrm{a}\left|\boldsymbol{\mu}_{\mathrm{m}, \mathrm{a}}\right| \mathrm{m}\right\rangle-\frac{i}{\hbar} \mathbf{E}(t) \cdot \sum_{\mathrm{n}}\left\langle\mathrm{a}\left|\boldsymbol{\mu}_{\mathrm{n}, \mathrm{a}}\right| \mathrm{n}\right\rangle \\
= & \frac{2}{\hbar} \operatorname{Re}\left\{\mathbf{E}(t) \cdot \sum_{\mathrm{n}}\left\langle\mathrm{a}\left|i \boldsymbol{\mu}_{\mathrm{n}, \mathrm{a}}\right| \mathrm{n}\right\rangle\right\}=-\frac{2}{\hbar} \operatorname{Im}\left\{\mathbf{E}(t) \cdot \sum_{\mathrm{n}}\left\langle\mathrm{a}\left|\boldsymbol{\mu}_{\mathrm{n}, \mathrm{a}}\right| \mathrm{n}\right\rangle\right\},
\end{aligned}
$$

pois para $z \in \mathbb{C}, z+z^{*}=2 \operatorname{Re}\{z\}$ e $(i z)+(i z)^{*}=-2 \operatorname{Im}\{z\}$.

Vamos daqui em diante trabalhar apenas na direção $z$ da representação das coordenadas, multiplicando a Eq. (2.54) pelo lado direito e pelo lado esquerdo por $\int_{-\infty}^{\infty} d^{3} \mathbf{r}|\mathbf{r}\rangle\langle\mathbf{r}|$ e utilizando separação de variáveis $\langle\mathbf{r} \mid \mathrm{n}\rangle=\Psi_{\mathrm{n}}(\mathbf{r})=\phi_{\mathrm{n}}(x) \varphi_{\mathrm{n}}(y) \psi_{\mathrm{n}}(z)$, de modo que

$$
\begin{aligned}
\mathbf{E}(t) & \rightarrow E_{\mathrm{z}}(t)=\varepsilon_{0} \cos \left(\omega_{\mathrm{f}} t\right) \\
\boldsymbol{\mu} & \rightarrow \mu=-e z
\end{aligned}
$$

onde $e$ é a carga elétrica elementar e $\omega_{\mathrm{f}}$ é a frequência angular do campo elétrico.

A integral da Eq. (2.57) ao longo de um intervalo de tempo $T_{0}$ fornece o número de elétrons atingindo ou deixando este nível durante este intervalo de tempo:

$$
\Delta N=\int_{0}^{T_{0}} d t \frac{d N_{\mathrm{a}}}{d t}
$$

Como dissemos acima, em um sistema sob ação de um campo eletromagnético, a absorção pode ser expressa como: $[9,11,14]$

$$
\sigma(\omega)=-\frac{\Delta N(\omega)}{N(\omega)} A
$$

onde $\omega$ é a frequência angular dos fótons incidentes e $N(\omega) / A$ é o número de fótons incidentes por unidade de área, que pode ser calculado como: [9]

$$
\frac{N(\omega)}{A}=\frac{|\tilde{\mathbf{E}}(\omega)|^{2} c}{\hbar \omega}
$$


onde $\tilde{\mathbf{E}}(\omega)$ é a Transformada de Fourier do campo elétrico ${ }^{4}$ :

$$
\begin{aligned}
\tilde{\mathbf{E}}(\omega) & =\frac{1}{2 \pi} \int_{-\infty}^{\infty} \mathbf{E}(t) e^{i \omega t} d t \\
& =\frac{1}{2 \pi} \int_{-\infty}^{\infty} \varepsilon_{0} \cos \left(\omega_{\mathrm{f}} t\right) e^{i \omega t} d t \\
& =\frac{1}{2 \pi} \int_{-\infty}^{\infty} \varepsilon_{0}\left(\frac{e^{i \omega_{\mathrm{f}} t}+e^{-i \omega_{\mathrm{f}} t}}{2}\right) e^{i \omega t} d t \\
& =\frac{\varepsilon_{0}}{2}\left[\frac{1}{2 \pi} \int_{-\infty}^{\infty} e^{i\left(\omega+\omega_{\mathrm{f}}\right) t} d t+\frac{1}{2 \pi} \int_{-\infty}^{\infty} e^{i\left(\omega-\omega_{\mathrm{f}}\right) t} d t\right] \\
& =\frac{\varepsilon_{0}}{2}\left[\delta\left(\omega+\omega_{\mathrm{f}}\right)+\delta\left(\omega-\omega_{\mathrm{f}}\right)\right] .
\end{aligned}
$$

Isso significa que se tivermos um campo elétrico monocromático com frequência $\omega_{\mathrm{f}}$, só estarão incidindo fótons de frequência $\omega_{\mathrm{f}}$, para todas as outras frequências a incidência é nula. Portanto, dado um campo elétrico monocromático de frequência $\omega_{\mathrm{f}}$, o número de fótons incidentes é dado por:

$$
\frac{N\left(\omega_{\mathrm{f}}\right)}{A}=\frac{\varepsilon_{0}^{2} c}{4 \hbar \omega_{\mathrm{f}}} .
$$

Quando avaliamos a integral da Eq. (2.59), a frequência do campo elétrico também é $\omega_{\mathrm{f}}$. Para um sistema de três níveis ${ }^{5}$, vamos calcular a absorção de fótons por elétrons no nível mais baixo $E_{0}$, através do número de elétrons excitados para os níveis $E_{1}$ e $E_{2}$. Fazemos isso utilizando as Eqs. (2.57), (2.59), (2.60), (2.62) e (2.63):

$$
\begin{aligned}
\sigma\left(\omega_{\mathrm{f}}\right)= & -\frac{8 e \omega_{\mathrm{f}}}{\varepsilon_{0} c} \int_{0}^{T_{0}} d t \operatorname{Im}\left\{\left\langle\Psi_{0} ; t|z| 1 ; t\right\rangle\left\langle\Psi_{0} ; t \mid 1 ; t\right\rangle\right\} \cos \left(\omega_{\mathrm{f}} t\right) \\
& -\frac{8 e \omega_{\mathrm{f}}}{\varepsilon_{0} c} \int_{0}^{T_{0}} d t \operatorname{Im}\left\{\left\langle\Psi_{0} ; t|z| 2 ; t\right\rangle\left\langle\Psi_{0} ; t \mid 2 ; t\right\rangle\right\} \cos \left(\omega_{\mathrm{f}} t\right),
\end{aligned}
$$

onde $\left|\Psi_{0} ; t\right\rangle=|0 ; t\rangle$ para $t=0$, mas $\left|\Psi_{0} ; t\right\rangle$ evolui com o hamiltoniano $\mathbf{H}$ da Eq. (2.53) enquanto que qualquer $|\mathrm{n} ; t\rangle$ evolui com o hamiltoniano $\mathbf{H}_{0}$ através da Eq. (2.10) em $t>0$.

Na Eq. (2.64) existe um pequeno abuso de linguagem pois o sanduíche já envolve apenas a direção $z$, rigorosamente o correto seria $z=r \cos (\theta)$. Sendo assim, vale a pena deixar claro que os sanduíches representam na verdade:

$$
\begin{aligned}
& \left\langle\Psi_{0} ; t \mid 1 ; t\right\rangle=\int_{-\infty}^{+\infty} d z \Psi_{0}^{*}(z, t) \psi_{1}(z) e^{-i E_{1} t / \hbar} \\
& \left\langle\Psi_{0} ; t|z| 1 ; t\right\rangle=\int_{-\infty}^{+\infty} d z \Psi_{0}^{*}(z, t) z \psi_{1}(z) e^{-i E_{1} t / \hbar} \\
& \left\langle\Psi_{0} ; t \mid 2 ; t\right\rangle=\int_{-\infty}^{+\infty} d z \Psi_{0}^{*}(z, t) \psi_{2}(z) e^{-i E_{2} t / \hbar} \\
& \left\langle\Psi_{0} ; t|z| 2 ; t\right\rangle=\int_{-\infty}^{+\infty} d z \Psi_{0}^{*}(z, t) z \psi_{2}(z) e^{-i E_{2} t / \hbar}
\end{aligned}
$$

\footnotetext{
$4 \quad$ Veja que $\omega$ é diferente de $\omega_{\mathrm{f}}$ na Eq. (2.58)

5 Escolhemos arbitrariamente um sistema de três níveis por ser o sistema que iremos estudar na Seção 5.5. Além disso, apesar de o sistema possuir 3 níveis discretos, existe um contínuo de níveis, acima do terceiro nível, que não iremos considerar.
} 
onde $\Psi_{0}(z, 0)=\psi_{0}(z)$ e $\psi_{0}(z), \psi_{1}(z)$ e $\psi_{2}(z)$ são autofunções do sistema, enquanto que $E_{1}$ e $E_{2}$ são as autoenergias do primeiro e segundo estado excitado, respectivamente.

A expressão (2.64) será utilizada para o cálculo do espectro de absorção no sistema analisado na Seção 5.5. É importante notar que precisaremos conhecer as autofunções $\psi_{0}(z), \psi_{1}(z)$ e $\psi_{2}(z)$ do sistema, bem como suas correspondes autoenergias. A evolução temporal destas autofunções pode ser obtida utilizando a Eq. (2.28), mas a evolução temporal de $\Psi_{0}(z, t)$ (que sofre influencia do campo elétrico) precisa ser realizada numericamente através das técnicas que apresentamos no Capítulo 4. 



\section{Heteroestruturas Semicondutoras}

Desde a idealização e fabricação das primeiras heteroestruturas semicondutoras (HS) $[15,16]$, na década de 1970, a produção destes sistemas evoluiu rapidamente com o surgimento e o aprimoramento das técnicas de crescimento de materiais semicondutores. Por heteroestruturas semicondutoras entendemos um material artificialmente produzido, o qual se forma pela superposição de camadas alternadas de semicondutores distintos. As modernas técnicas de crescimento de materiais semicondutores, tais como, MBE [17], CBE [18] (Chemical Beam Epitaxy) e MOCVD [19] (Metalorganic Chemical Vapour Deposition) permitem atualmente a produção de amostras monocristalinas com um número preciso de camadas atômicas, interfaces abruptas, perfis de dopagem sofisticados, alta pureza e baixo número de defeitos estruturais. Heteroestruturas semicondutoras crescidas em diversas arquiteturas formam a base de uma série de dispositivos eletrônicos, ópticos e opto-eletrônicos e representam sistemas extremamente interessantes do ponto de vista da Física Básica [11, 12].

Durante um longo período, o princípio utilizado para a fabricação de heteroestruturas semicondutoras foi o crescimento de materiais de mesmo parâmetro de rede (ou bastante próximo) que o substrato utilizado. Uma enorme variedade de HS foram produzidas, mudando-se a natureza, a composição, a espessura e a própria sequência das camadas epitaxiais durante o crescimento. Na década de 1980, foi demonstrado que o crescimento de estruturas tensionadas, cujo parâmetro de rede era significativamente diferente daquele do substrato, podia fornecer sistemas físicos de grande interesse tecnológico, por exemplo, o crescimento de pontos quânticos.[20] Materiais semicondutores sob tensão deram origem a uma nova linha de pesquisa quando, em 1982, Osbourn mostrou que a tensão elástica modificava a estrutura de bandas do semicondutor e sugeriu que este fato poderia ser útil tecnologicamente.[21] O princípio básico da epitaxia de camadas sob tensão consiste na possibilidade de um material (com parâmetro de rede diferente daquele do substrato) acomodar seu parâmetro de rede ao do substrato mantendo a mesma estrutura cristalina.

Um desses materiais produzidos por técnicas de crescimento de materiais semicondutores sob tensão e que merece destaque por sua enorme aplicação tecnológica é a liga ternária de $\operatorname{In}_{x} \mathrm{Ga}_{1-x} \mathrm{As}$, formada a partir dos compostos binários InAs e GaAs. Devido à diferença entre os parâmetros de rede do InAs e do GaAs, o crescimento da liga de $\operatorname{In}_{x} \mathrm{Ga}_{1-x} \mathrm{As}$ sobre um substrato de GaAs é realizado sob tensão, que altera de maneira significativa a estrutura eletrônica do material tensionado. Hoje, poços quânticos de $\operatorname{In}_{x} \mathrm{Ga}_{1-x} \mathrm{As}$ sob tensão são muito importantes para as indústrias micro e optoeletrônicas, uma vez que esses poços podem ser usados em uma ampla gama de dispositivos de alto desempenho, como lasers operando na região de $483 \mathrm{~nm}$ [22], por exemplo. Além 
da tensão, atualmente está bem estabelecido que o crescimento de camadas de InGaAs sobre substratos de GaAs ocorre com uma forte segregação de átomos de índio, que se acumulam na frente de crescimento, resultando em um gradiente da composição de índio na direção de crescimento.[23, 24] Esse fenômeno modifica os perfis de potencial das heteroestruturas semicondutoras dando origem a diferentes propriedades eletrônicas dos dispositivos produzidos com base nesse material.

Neste capítulo, iremos introduzir as principais ideias que sustentam nosso entendimento das heteroestruturas semicondutoras pressupondo familiares alguns conceitos de Mecânica Quântica, que foram aprofundados de forma mais seletiva no Capítulo 2.

\subsection{Aproximação da Massa Efetiva em Materiais Semicondutores}

Neste trabalho vamos dar enfoque aos semicondutores cristalinos, nos quais os átomos estão distribuídos de forma regular no espaço, formando um arranjo periódico. No átomo isolado, os elétrons dividem-se em níveis discretos de energia correspondendo aos orbitais atômicos designados por 1s, 2s, 2p, 3s, 3d, etc. No cristal, por causa da interação entre os átomos, esses níveis se alargam em bandas de energia permitidas separadas por bandas de energia proibidas, como na Figura 3 que mostra a estrutura de bandas do GaAs.

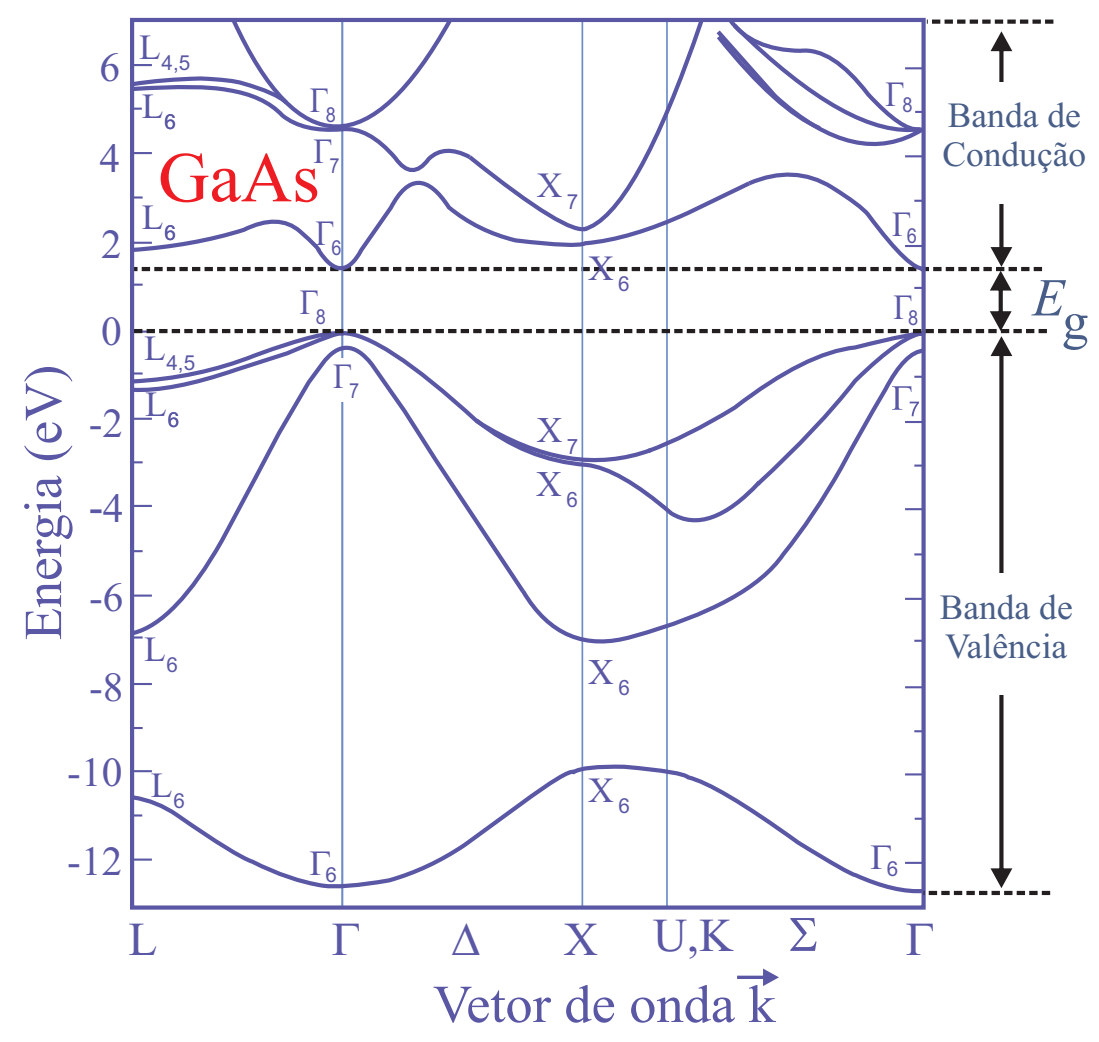

Figura 3: Estrutura de bandas de energia do GaAs (figura copiada da referência [25]). 
A banda superior é denominada banda de condução (BC), pois representa os estados eletrônicos excitados que participam do fluxo de corrente. Os elétrons que a ocupam são os promovidos da banda inferior devido a aumento de temperatura, ou excitação ótica, ou por dopagem, por exemplo. A banda inferior é conhecida como banda de valência (BV), e se origina nos estados dos elétrons de valência que constituem as ligações covalentes que unem os átomos do cristal. A região entre as bandas de valência e condução é chamada de faixa de energia proibida e a energia mínima entre estas duas bandas é chamada de energia do $\boldsymbol{g a p}\left(E_{\mathrm{g}}\right)$. Esta é a energia mínima necessária para liberar um elétron da banda de valência (onde o elétron está preso a um átomo) para a banda de condução (onde ele pode se mover pelo material). Em um cristal puro, no zero absoluto, o valor de $E_{\mathrm{g}}$ é bem conhecido, mas para temperaturas finitas, para cristais não puros ou mesmo quando crescidos sobre outros cristais, este valor sofre alterações.

Em $T=0 \mathrm{~K}$, a banda de valência é completamente cheia, enquanto que a banda de condução é vazia. Se o cristal é aquecido, um pequeno número (n) de elétrons pode ser excitado para a banda de condução, deixando lugares vazios (um número p) na banda de valência, denominados buracos. De acordo com o mecanismo de criação desses elétrons e buracos, tem-se obviamente que $n=p=n_{\mathrm{i}}$, chamada de concentração intrínseca. A variação da concentração intrínseca com a temperatura é dada pela lei de MaxwellBoltzmann

$$
n_{\mathrm{i}}=n_{0} \exp \left(-\frac{E_{\mathrm{g}}}{2 k_{B} T}\right)
$$

onde $T$ é a temperatura absoluta, $k_{B}$ a constante de Boltzmann e $n_{0}$ uma constante característica do semicondutor. Na temperatura ambiente $(T=300 \mathrm{~K}) n_{\mathrm{i}}=10^{10}$ pares de elétron-buraco por centímetro cúbico para o silício, e $10^{13}$ por centímetro cúbico para o germânio. Esses são valores notavelmente pequenos, já que um cristal de germânio tem em torno de $10^{22}$ átomos por centímetro cúbico; há, portanto, um elétron intrínseco (ou excitado) para cada bilhão $\left(10^{9}\right)$ de átomos.

A estrutura de bandas é geralmente representada como uma dispersão de energias $E$ em função do vetor de onda $\overrightarrow{\mathbf{k}}$, que está relacionado ao momento dos elétrons. O GaAs (assim como AlAs e o InAs) é um semicondutor com gap direto, isso significa que para que um elétron no topo da banda de valência possa se libertar ele só precisa receber a energia de um fóton com energia maior ou igual ao gap. Alguns semicondutores como o silício e o germânio possuem gap indireto, onde o máximo da banda de valência não está no mesmo ponto $\overrightarrow{\mathbf{k}}$ do fundo da banda de condução. Nestes casos, além de energia, o elétron precisa de uma mudança em seu vetor de onda, ou seja, uma mudança de momento cristalino $\hbar \overrightarrow{\mathbf{k}}$. Este momento pode ser cedido ao elétron por meio de uma vibração da rede cristalina, denominada fônon.[26]

Nos materiais semicondutores de gap direto, a maioria dos processos de excitação de elétrons ocorre das proximidades do topo da banda de valência para o fundo da banda 


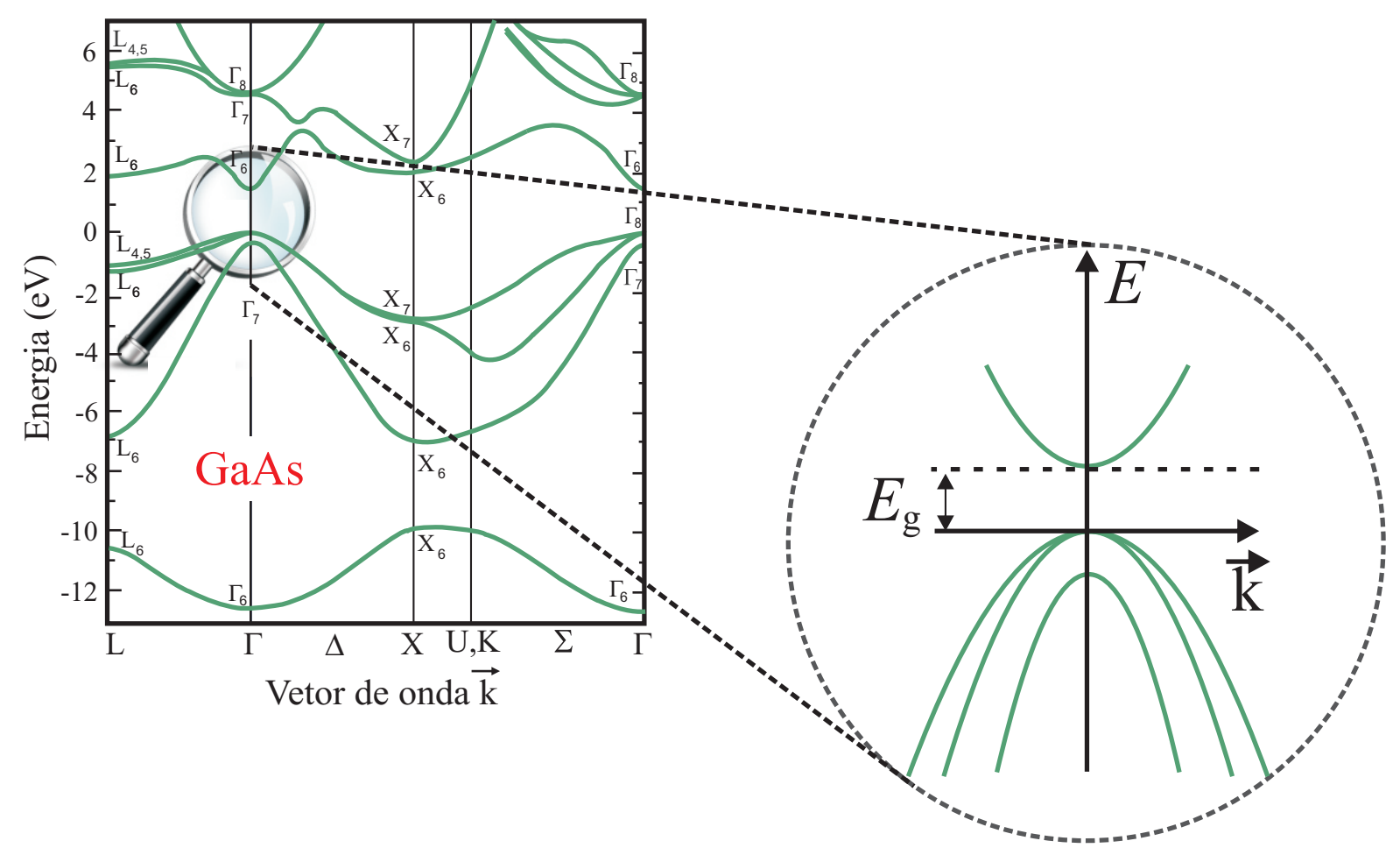

Figura 4: Para pequenos deslocamentos em $\overrightarrow{\mathbf{k}}$, a dispersão de energias em função de $\overrightarrow{\mathbf{k}}$ é aproximadamente quadrática, principal condição utilizada na aproximação da massa efetiva.

de condução. Por esta razão quase sempre iremos trabalhar em limiares em que esta condição seja relevante. Nesse contexto surge uma aproximação muito importante conhecida como aproximação da massa efetiva. O ponto de partida para esta aproximação é supor que nas vizinhanças do fundo da banda de condução e do topo da banda de valência a dispersão de energias em função dos vetores de onda $\overrightarrow{\mathbf{k}}$ é aproximadamente quadrática, como na Figura 4.

Se o topo da banda de valência for utilizado como referência, a energia de um estado eletrônico na banda de condução será aproximadamente da forma

$$
E_{\overrightarrow{\mathbf{k}}} \approx E_{\mathrm{g}}+\alpha \overrightarrow{\mathbf{k}}^{2}
$$

dispersão esta que se assemelha à de uma partícula livre de massa $m$ [26]

$$
E_{\text {livre }}=\frac{\hbar^{2} \overrightarrow{\mathbf{k}}^{2}}{2 m}
$$

Mas como um cristal não é uma região de potencial nulo onde uma partícula pode se mover livremente, supõe-se que a partícula tem uma massa um pouco menor, chamada massa efetiva $m^{*}$.[12] É possível mostrar que essa aproximação é fortemente sustentada pelas velocidades de fase e de grupo de elétrons movendo-se no interior da rede cristalina em razão de uma força externa, como um campo eletromagnético.[26] Aqui vamos nos limitar a escrever diretamente

$$
E_{\overrightarrow{\mathbf{k}}}=E_{\mathrm{g}}+\frac{\hbar^{2} \overrightarrow{\mathbf{k}}^{2}}{2 m^{*}}
$$


onde assumimos a total isotropia do tensor de massa na região em que $k \approx 0$.

Se escrevermos a função de onda de um elétron como uma combinação de funções de Bloch com a periodicidade da rede cristalina (isto é, que $u_{\overrightarrow{\mathbf{k}}}(\overrightarrow{\mathbf{r}})=u_{\overrightarrow{\mathbf{k}}}(\mathbf{r}+\mathbf{R})$ onde $\overrightarrow{\mathbf{r}}$ é um vetor de translação da rede) e levando em conta a dependência temporal [12]

$$
\Psi(\overrightarrow{\mathbf{r}}, t)=\sum_{\overrightarrow{\mathbf{k}}} c_{\overrightarrow{\mathbf{k}}} u_{\overrightarrow{\mathbf{k}}}(\overrightarrow{\mathbf{r}}) \exp (i \overrightarrow{\mathbf{k}} \cdot \overrightarrow{\mathbf{r}}) \exp \left(-\frac{i E_{\overrightarrow{\mathbf{k}}} t}{\hbar}\right)
$$

podemos assumir que $u_{\overrightarrow{\mathbf{k}}}(\overrightarrow{\mathbf{r}}) \approx u_{0}(\overrightarrow{\mathbf{r}})$, de modo que

$$
\Psi(\overrightarrow{\mathbf{r}}, t)=u_{0}(\overrightarrow{\mathbf{r}})\left[\sum_{\overrightarrow{\mathbf{k}}} c_{\overrightarrow{\mathbf{k}}} \exp (i \overrightarrow{\mathbf{k}} \cdot \overrightarrow{\mathbf{r}}) \exp \left(-\frac{i E_{\overrightarrow{\mathbf{k}}} t}{\hbar}\right)\right]=u_{0}(\overrightarrow{\mathbf{r}}) \widetilde{\Psi}(\overrightarrow{\mathbf{r}}, t) .
$$

A função de onda $\Psi(\overrightarrow{\mathbf{r}}, t)$ da Eq. (3.6) precisa obedecer a equação de Schrödinger dependente do tempo

$$
\begin{aligned}
i \hbar \frac{\partial \Psi(\overrightarrow{\mathbf{r}}, t)}{\partial t} & =i \hbar \frac{\partial}{\partial t}\left\{u_{0}(\overrightarrow{\mathbf{r}})\left[\sum_{\overrightarrow{\mathbf{k}}} c_{\overrightarrow{\mathbf{k}}} \exp (i \overrightarrow{\mathbf{k}} \cdot \overrightarrow{\mathbf{r}}) \exp \left(-\frac{i E_{\overrightarrow{\mathbf{k}}} t}{\hbar}\right)\right]\right\} \\
& =u_{0}(\overrightarrow{\mathbf{r}})\left[\sum_{\overrightarrow{\mathbf{k}}} E_{\overrightarrow{\mathbf{k}}} c_{\overrightarrow{\mathbf{k}}} \exp (i \overrightarrow{\mathbf{k}} \cdot \overrightarrow{\mathbf{r}}) \exp \left(-\frac{i E_{\overrightarrow{\mathbf{k}}} t}{\hbar}\right)\right] \\
& =u_{0}(\overrightarrow{\mathbf{r}})\left[\sum_{\overrightarrow{\mathbf{k}}}\left(E_{g}+\frac{\hbar^{2} \overrightarrow{\mathbf{k}}^{2}}{2 m^{*}}\right) c_{\overrightarrow{\mathbf{k}}} \exp (i \overrightarrow{\mathbf{k}} \cdot \overrightarrow{\mathbf{r}}) \exp \left(-\frac{i E_{\overrightarrow{\mathbf{k}}} t}{\hbar}\right)\right] \\
& =u_{0}(\overrightarrow{\mathbf{r}})\left[E_{\mathrm{g}} \widetilde{\Psi}(\overrightarrow{\mathbf{r}}, t)+\frac{\hbar^{2}}{2 m^{*}} \sum_{\overrightarrow{\mathbf{k}}} c_{\overrightarrow{\mathbf{k}}} \overrightarrow{\mathbf{k}}^{2} \exp (i \overrightarrow{\mathbf{k}} \cdot \overrightarrow{\mathbf{r}}) \exp \left(-\frac{i E_{\overrightarrow{\mathbf{k}}} t}{\hbar}\right)\right] \\
& =u_{0}(\overrightarrow{\mathbf{r}})\left[E_{\mathrm{g}} \widetilde{\Psi}(\overrightarrow{\mathbf{r}}, t)+\frac{\hbar^{2}}{2 m^{*}} \sum_{\overrightarrow{\mathbf{k}}} c_{\overrightarrow{\mathbf{k}}}\left(-\vec{\nabla}_{\overrightarrow{\mathbf{r}}}^{2} \exp (i \overrightarrow{\mathbf{k}} \cdot \overrightarrow{\mathbf{r}})\right) \exp \left(-\frac{i E_{\overrightarrow{\mathbf{k}}} t}{\hbar}\right)\right] \\
& =u_{0}(\overrightarrow{\mathbf{r}})\left[E_{\mathrm{g}} \widetilde{\Psi}(\overrightarrow{\mathbf{r}}, t)-\frac{\hbar^{2}}{2 m^{*}} \vec{\nabla}_{\overrightarrow{\mathbf{r}}}^{2} \sum_{\overrightarrow{\mathbf{k}}} c_{\overrightarrow{\mathbf{k}}} \exp (i \overrightarrow{\mathbf{k}} \cdot \overrightarrow{\mathbf{r}}) \exp \left(-\frac{i E_{\overrightarrow{\mathbf{k}}} t}{\hbar}\right)\right] \\
& =u_{0}(\overrightarrow{\mathbf{r}})\left[E_{\mathrm{g}} \widetilde{\Psi}(\overrightarrow{\mathbf{r}}, t)-\frac{\hbar^{2}}{2 m^{*}} \vec{\nabla}_{\overrightarrow{\mathbf{r}}}^{2} \widetilde{\Psi}(\overrightarrow{\mathbf{r}}, t)\right]
\end{aligned}
$$

Substituindo a expressão (3.6) no lado esquerdoo de (3.7) e cancelando $u_{0}(\overrightarrow{\mathbf{r}})$ em ambos os lados desta expressão obtemos:

$$
i \hbar \frac{\partial \widetilde{\Psi}(\overrightarrow{\mathbf{r}}, t)}{\partial t}=-\frac{\hbar^{2}}{2 m^{*}} \vec{\nabla}_{\overrightarrow{\mathbf{r}}}^{2} \widetilde{\Psi}(\overrightarrow{\mathbf{r}}, t)+E_{\mathrm{g}} \widetilde{\Psi}(\overrightarrow{\mathbf{r}}, t) .
$$

A função de onda $\widetilde{\Psi}(\overrightarrow{\mathbf{r}}, t)$ recebe o nome de função envelope e através das equações (3.6) e (3.8) vemos que ela obedece a ESDT para uma partícula de massa efetiva $m^{*}$ que pode assumir os mesmos níveis de energia que um elétron próximo ao fundo da banda de condução. Apesar de $E_{\mathrm{g}}$ ter sido considerada constante na Eq. (3.8), esta abordagem permanece válida mesmo para $E_{\mathrm{g}}(\overrightarrow{\mathbf{r}})$ variando abruptamente no espaço.[12] 
Em heteroestruturas semicondutoras, cristais diferentes são crescidos de forma adjacente, de forma que o gap e a massa efetiva variam espacialmente. Nessas condições, a função envelope deve satisfazer [7, 11, 12]

$$
i \hbar \frac{\partial \widetilde{\Psi}(\overrightarrow{\mathbf{r}}, t)}{\partial t}=-\frac{\hbar^{2}}{2} \vec{\nabla}_{\overrightarrow{\mathbf{r}}}\left(\frac{1}{m^{*}(\overrightarrow{\mathbf{r}})} \vec{\nabla}_{\overrightarrow{\mathbf{r}}} \widetilde{\Psi}(\overrightarrow{\mathbf{r}}, t)\right)+E_{\mathrm{g}}(\overrightarrow{\mathbf{r}}) \widetilde{\Psi}(\overrightarrow{\mathbf{r}}, t),
$$

que se reduz à Eq. (3.8) quando $E_{\mathrm{g}}$ e $m^{*}$ não possuem dependência espacial. Ao longo deste trabalho, sempre que estivermos buscando as soluções da ESDT estaremos nos referindo às funções envelope das equações (3.8) e (3.9).

\subsection{Poços Quânticos Crescidos por MBE}

A técnica de Epitaxia por Feixe Molecular [17] permite a criação de filmes finos a partir da deposição controlada de monocamadas cristalinas ${ }^{1}$. Este processo ocorre em ultra alto vácuo ( 10 $10^{-12}$ Torr), quando elementos como In, Ga, Al e As provenientes de fontes de alta pureza (localizadas nas células de efusão) são sublimados e direcionados para uma superfície inicial chamada de substrato. Ao atingir a superfície, os átomos reagem entre si e acabam se acomodando de forma a repetir a estrutura cristalina do substrato. Por sinal, o substrato é em geral um cristal puro de algum dos semicondutores envolvidos no processo, por exemplo GaAs.[17]

Além de permitir o crescimento de um número conhecido de monocamadas cristalinas, o MBE também permite uma alternância de materiais, ou seja, é possível crescer um determinado número de monocamadas de GaAs, alternar o tipo de material para InGaAs, crescer algum outro número de monocamadas, para finalmente voltar a crescer monocamadas de GaAs. Estruturas como estas recebem o nome de poços quânticos.

Na Figura 5 mostramos o perfil de potencial de um poço quântico do tipo AlGaAs/GaAs. Na parte superior é mostrado um sólido representando as camadas semicondutoras que compõem a heteroestrutura, onde é importante notar que a direção $z$ (do crescimento) possui dimensão de angstron, enquanto que as direções $x$ e $y$ têm dimensões de centímetros. O marcador $N_{1} a_{\mathrm{AlGaAs}}$ indica a existência de $N_{1}$ monocamadas com parâmetro de rede do AlGaAs ${ }^{2}$, implicando que o comprimento em $\AA$ dessa região é $N_{1} \times a_{\mathrm{AlGaAs}} / 2$, onde $a_{\mathrm{AlGaAs}}$ é o parâmetro de rede da célula unitária. O mesmo valendo para $N_{2} a_{\mathrm{GaAs}}$ e $N_{3} a_{\text {AlGaAs. }}$.

Inicialmente o valor zero de energia é adotado como sendo o topo da banda de valência do material que compõem o substrato, no caso da Figura 5, o AlGaAs. Entretanto,

1 Refere-se a cada camada crescida pela técnica de MBE como monocamada (MC), ou as vezes pelo termo em inglês monolayer (ML)

2 É importante ressaltar que uma monocamada de um cristal do tipo blenda de zinco tem na verdade a altura de meio parâmetro de rede. 


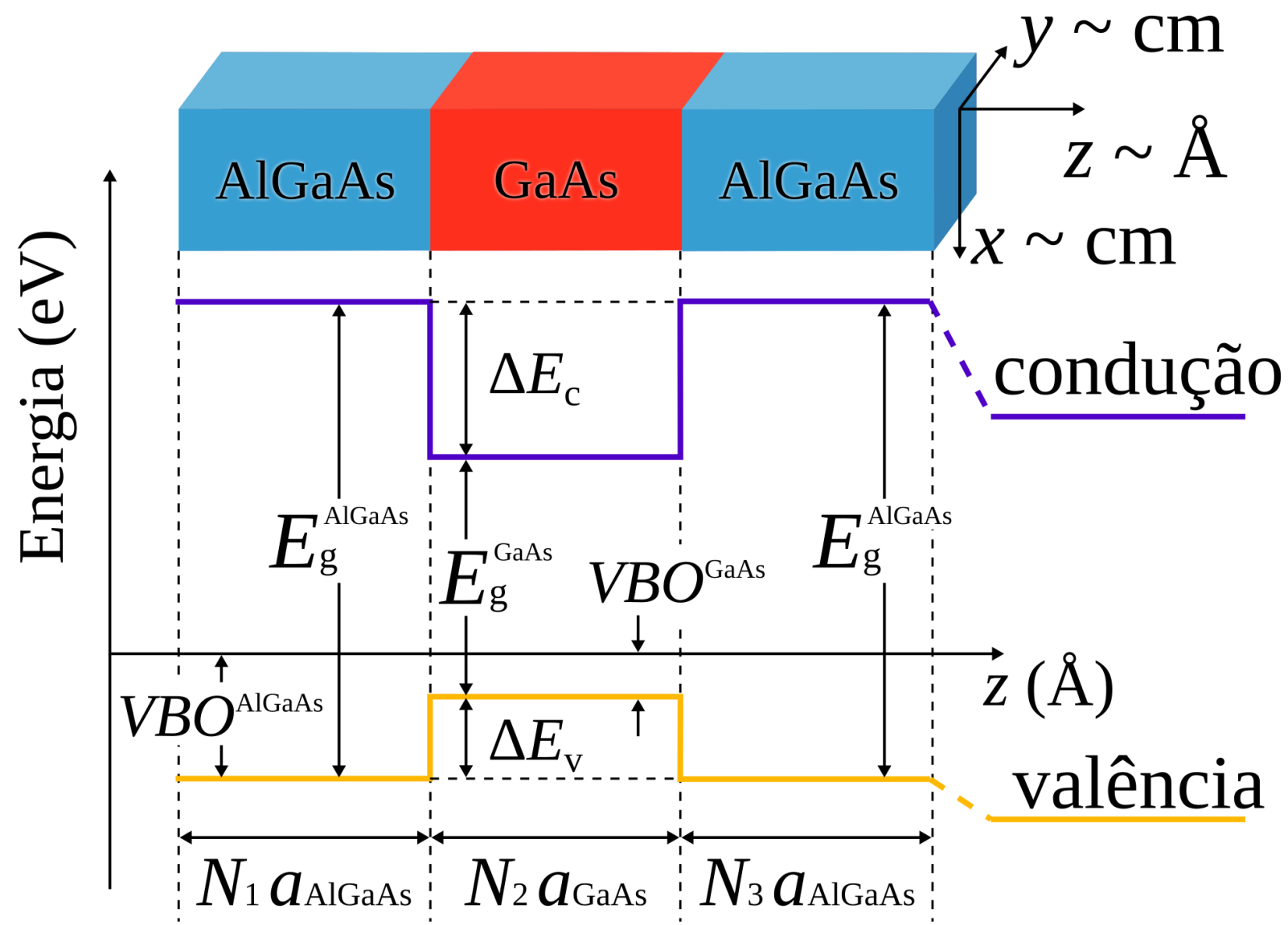

Figura 5: Perfil de potencial criado dentro de uma estrutura do tipo poço quântico de AlGaAs/GaAs.

cada liga ou material possui um parâmetro chamado VBO (do inglês, Valence Band Offset) que determina um valor em energia em que o topo da banda de valência deve ser deslocado.[7, 11, 48] Como para o AlGaAs este valor é de aproximadamente $V B O^{\mathrm{AlGaAs}}=$ $-1,33 \mathrm{eV}$ e para o GaAs ele é de aproximadamente $V B O^{\mathrm{GaAs}}=-0,8 \mathrm{eV}$, acontece este desvio no alinhamento das bandas de valência e de condução.[48]

Para que um elétron esteja na banda de condução na primeira camada da heteroestrutura, no lado esquerdo, ele precisa ter uma energia maior ou igual à energia do gap do AlGaAs $\left(E_{\mathrm{g}}^{\mathrm{AlGaAs}}\right)$ somada ao $V B O^{\mathrm{AlGaAs}}$, o mesmo valendo para um elétron na última camada do lado direito. Entretanto, um elétron na região central da heteroestrutura, que possua uma energia maior do que a energia do gap do GaAs ( $E_{\mathrm{g}}^{\mathrm{GaAs}}$ ) somada ao $V B O^{\mathrm{GaAs}}$, pode se deslocar nesta região, mas não consegue se mover para as camadas adjacentes de AlGaAs. O tamanho diminuto da região central na Figura 5, comparável com o comprimento de onda do elétron, propicia o surgimento de níveis quantizados de energia na direção $z$, completamente análogo aos poços quânticos apresentados nos textos de Mecânica Quântica, devendo-se apenas levar em consideração a aproximação da massa efetiva, esquematizada na Eq. (3.9) para o caso tridimensional, mas que pode ser reduzida de forma trivial para o caso unidimensional. O último ponto que precisa ser reforçado aqui é que com a quantização ocorrendo somente na direção $z$, o elétron ainda pode se 


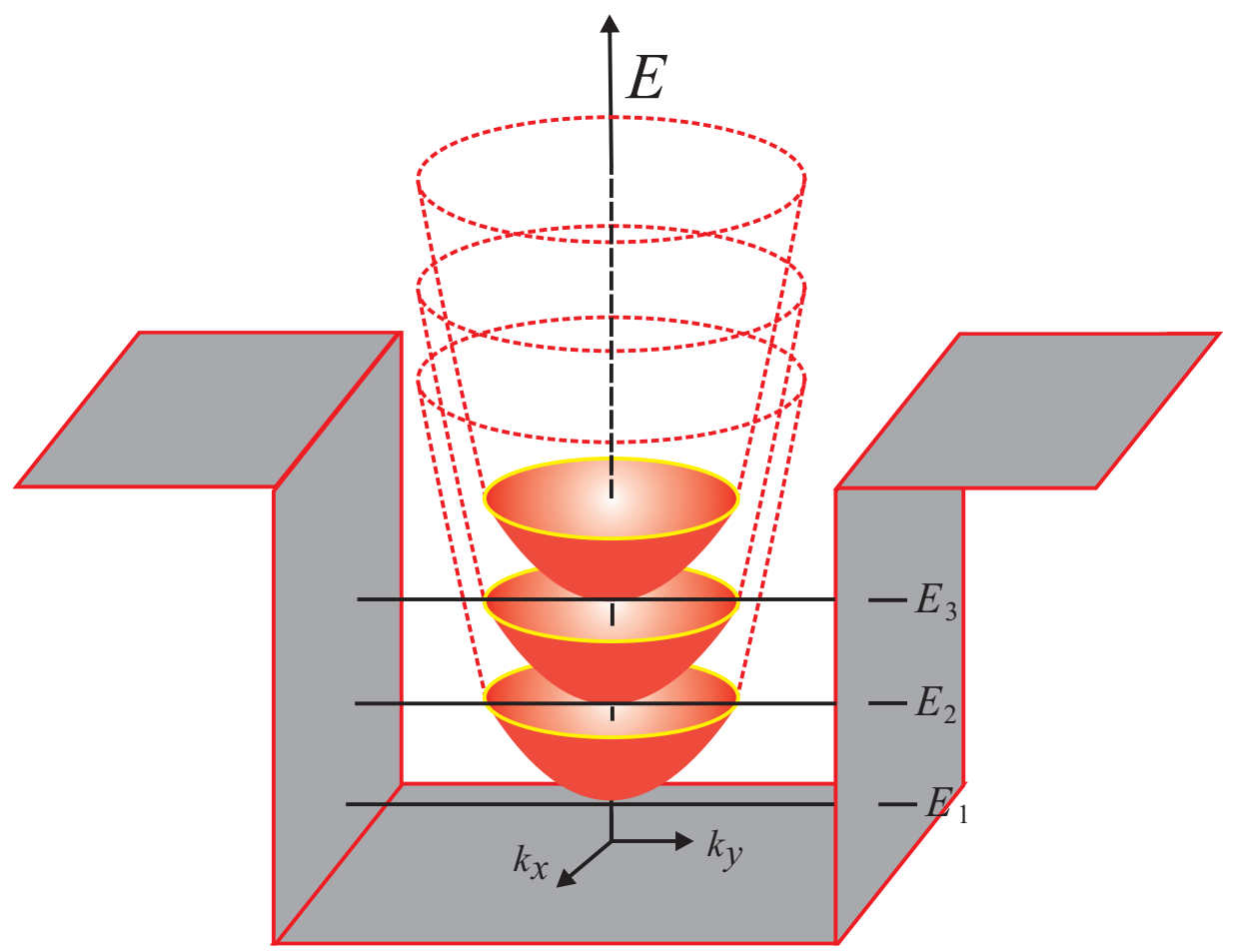

Figura 6: Forma das bandas de energia em um poço quântico, evidenciando a relação quadrática de $E$ com $k_{\mathrm{x}}$ e $k_{\mathrm{y}} . E_{1}, E_{2}$ e $E_{3}$ representam 3 níveis de confinamento no poço.

comportar como uma partícula livre de massa $m^{*}$ nas direções $x$ e $y$. Sendo assim, quando resolvermos a ESIT da função envelope no caso unidimensional, obteremos alguns níveis discretos de energia $E_{\mathrm{n}}$, mas os elétrons nestas condições podem assumir os seguintes níveis de energia:

$$
\begin{aligned}
E_{\mathrm{n}}, & =\left(E_{\mathrm{g}}^{\mathrm{GaAs}}+V B O^{\mathrm{GaAs}}\right)+E_{\mathrm{n}}+\frac{\hbar^{2} k_{\mathrm{x}}^{2}}{2 m^{*}}+\frac{\hbar^{2} k_{\mathrm{y}}^{2}}{2 m^{*}} \\
& =\left(E_{\mathrm{g}}^{\mathrm{GaAs}}+V B O^{\mathrm{GaAs}}\right)+E_{\mathrm{n}}+\frac{\hbar^{2}\left(k_{\mathrm{x}}^{2}+k_{\mathrm{y}}^{2}\right)}{2 m^{*}} .
\end{aligned}
$$

A Eq. (3.10) implica que, dentro do poço quântico, a estrutura de bandas é alterada, reduzindo o número de estados possíveis, como indicado na Figura 6.[11, 12] 


\section{Modelagem Numérica da Equação de Schrödinger}

Vários métodos numéricos já foram desenvolvidos e empregados em diferentes aproximações, com a finalidade de encontrar os estados quânticos.[1, 27, 28, 29, 30, 31, 32] Nesta dissertação estamos interessados não só em encontrar os estados quânticos, mas também em obter sua evolução temporal.

Para o problema de evolução temporal utilizamos técnicas de resolução de equações diferenciais parciais baseadas no Método das Diferenças Finitas e um outro método numérico baseado no operador quântico de evolução temporal chamado de Método Pseudo-Espectral ou Método de Fourier.[1, 9, 33] Já para o problema de encontrar os estados quânticos utilizamos o método da Iteração Inversa.[34, 35]

Qualquer método de evolução temporal pode também ser utilizado para encontrar os estados quânticos através de um processo chamado de evolução em tempo imaginário, onde uma transformação algébrica faz com que qualquer estado convirja para o estado fundamental após um longo período de tempo, sendo os estados excitados obtidos através de ortogonalização de Gram-Schmidt.[6, 24, 33]

Neste capítulo introduziremos os métodos citados acima, para servir de referência prática às aplicações realizadas nos capítulos seguintes.

\subsection{Método das Diferenças Finitas}

A ESDT para uma única partícula em uma dimensão é uma equação diferencial do tipo parabólica.[35] Para resolver esta equação numericamente, o primeiro passo é introduzir uma malha de pontos equidistantes no espaço, separados pela distância $\Delta z$, sobre a qual determinaremos a função de onda. É possível modificar esta escolha, e utilizar pontos que não sejam equidistantes, algo que em princípio permite uma concentração maior de pontos em regiões de interesse, sem aumentar o número total de pontos, mas observamos que isso acaba aumentando o tempo de convergência para alguns casos de interesse, como potenciais inclinados por um campo elétrico constante. O tempo também será discretizado, ou seja, a função de onda será calculada em alguns valores discretos de $t$ separados pelo intervalo $\Delta t$. Assim, a função $\Psi(z, t)$ será representada por um conjunto discreto de valores $\Psi_{\mathrm{j}}^{\mathrm{k}}$, onde o índice $\mathrm{j}$ indica o j-ésimo ponto no espaço e $\mathrm{k}$ indica o k-ésimo instante de tempo. Especificamente, para uma região na direção $z$ de tamanho 
$L$, amostrada em $N$ pontos, a malha será dada por:

$$
z_{\mathrm{j}}=-\frac{L}{2}+\mathrm{j}\left(\frac{L}{N-1}\right)=-\frac{L}{2}+\mathrm{j} \Delta z, \mathrm{j}=0, \ldots, N-1
$$

e, adotando o instante inicial como sendo $t=0$, o tempos discretos serão dados por

$$
t_{\mathrm{k}}=\mathrm{k} \Delta t, \mathrm{k}=0,1,2, \ldots
$$

O próximo passo é aproximar as derivadas na equação de Schrödinger por diferenças finitas. Elas devem fornecer valores aproximados das respectivas derivadas desde que $\Delta z$ e $\Delta t$ sejam suficientemente pequenos. Uma primeira aproximação para a derivada no tempo é dada por:

$$
\frac{\partial}{\partial t} \Psi(z, t) \approx \frac{\Psi(z, t+\Delta t)-\Psi(z, t)}{\Delta t} \equiv \frac{\Psi_{\mathrm{j}}^{\mathrm{k}+1}-\Psi_{\mathrm{j}}^{\mathrm{k}}}{\Delta t}
$$

que torna-se exata quando $\Delta t \rightarrow 0$. Para as derivadas parciais no espaço, podemos considerar as diferenças finitas progressivas, retroativas ou centrais, equações (4.4), (4.5) e (4.6), respectivamente,

$$
\begin{gathered}
\frac{\partial}{\partial z} \Psi(z, t) \approx \frac{\Psi(z+\Delta z, t)-\Psi(z, t)}{\Delta z} \equiv \frac{\Psi_{\mathrm{j}+1}^{\mathrm{k}}-\Psi_{\mathrm{j}}^{\mathrm{k}}}{\Delta z}, \\
\frac{\partial}{\partial z} \Psi(z, t) \approx \frac{\Psi(z, t)-\Psi(z-\Delta z, t)}{\Delta z} \equiv \frac{\Psi_{\mathrm{j}}^{\mathrm{k}}-\Psi_{\mathrm{j}-1}^{\mathrm{k}}}{\Delta z}, \\
\frac{\partial}{\partial z} \Psi(z, t) \approx \frac{\Psi(z+\Delta z, t)-\Psi(z-\Delta z, t)}{2 \Delta z} \equiv \frac{\Psi_{\mathrm{j}+1}^{\mathrm{k}}-\Psi_{\mathrm{j}-1}^{\mathrm{k}}}{2 \Delta z} .
\end{gathered}
$$

A escolha do tipo de diferenças finitas varia de acordo com o tipo de esquema adotado (implícito ou explícito) como veremos adiante.

Derivadas parciais de ordens superiores são calculadas de forma semelhante, também podendo ser progressivas, retroativas ou centrais, como nas equações (4.7), (4.8) e (4.9), respectivamente:

$$
\begin{aligned}
& \frac{\partial^{2}}{\partial z^{2}} \Psi(z, t) \approx \frac{\Psi(z+2 \Delta z, t)-2 \Psi(z+\Delta z, t)+\Psi(z, t)}{\Delta z^{2}} \equiv \frac{\Psi_{\mathrm{j}+2}^{\mathrm{k}}-2 \Psi_{\mathrm{j}+1}^{\mathrm{k}}+\Psi_{\mathrm{j}}^{\mathrm{k}}}{\Delta z^{2}} \\
& \frac{\partial^{2}}{\partial z^{2}} \Psi(z, t) \approx \frac{\Psi(z, t)-2 \Psi(z-\Delta z, t)+\Psi(z-2 \Delta z, t)}{\Delta z^{2}} \equiv \frac{\Psi_{\mathrm{j}}^{\mathrm{k}}-2 \Psi_{\mathrm{j}-1}^{\mathrm{k}}+\Psi_{\mathrm{j}-2}^{\mathrm{k}}}{\Delta z^{2}} \\
& \frac{\partial^{2}}{\partial z^{2}} \Psi(z, t) \approx \frac{\Psi(z+\Delta z, t)-2 \Psi(z, t)+\Psi(z-\Delta z, t)}{\Delta z^{2}} \equiv \frac{\Psi_{\mathrm{j}+1}^{\mathrm{k}}-2 \Psi_{\mathrm{j}}^{\mathrm{k}}+\Psi_{\mathrm{j}-1}^{\mathrm{k}}}{\Delta z^{2}} .
\end{aligned}
$$


Utilizando diferenças finitas centrais no espaço e progressivas no tempo, podemos escrever a equação de Schrödinger como

$$
i \hbar\left(\frac{\Psi_{\mathrm{j}}^{\mathrm{k}+1}-\Psi_{\mathrm{j}}^{\mathrm{k}}}{\Delta t}\right)=-\frac{\hbar^{2}}{2 m}\left(\frac{\Psi_{\mathrm{j}+1}^{\mathrm{k}}-2 \Psi_{\mathrm{j}}^{\mathrm{k}}-\Psi_{\mathrm{j}-1}^{\mathrm{k}}}{\Delta z^{2}}\right)+V_{\mathrm{j}}^{\mathrm{k}} \Psi_{\mathrm{j}}^{\mathrm{k}} .
$$

Vale a pena ainda definir

$$
\frac{d \Psi_{\mathrm{j}}^{\mathrm{k}}}{d t} \equiv \frac{\Psi_{\mathrm{j}}^{\mathrm{k}+1}-\Psi_{\mathrm{j}}^{\mathrm{k}}}{\Delta t}=\frac{i \hbar}{2 m}\left(\frac{\Psi_{\mathrm{j}+1}^{\mathrm{k}}-2 \Psi_{\mathrm{j}}^{\mathrm{k}}-\Psi_{\mathrm{j}-1}^{\mathrm{k}}}{\Delta z^{2}}\right)-\frac{i V_{\mathrm{j}}^{\mathrm{k}}}{\hbar} \Psi_{\mathrm{j}}^{\mathrm{k}} .
$$

Supondo que conhecemos a função de onda em uma dado instante, é possível determiná-la em um instante seguinte. Para isso, temos apenas que resolver a Eq. (4.10) $\operatorname{para} \Psi_{\mathrm{j}}^{\mathrm{k}+1}$

$$
\begin{aligned}
\Psi_{\mathrm{j}}^{\mathrm{k}+1} & =\frac{i \hbar \Delta t}{2 m}\left(\frac{\Psi_{\mathrm{j}+1}^{\mathrm{k}}-2 \Psi_{\mathrm{j}}^{\mathrm{k}}-\Psi_{\mathrm{j}-1}^{\mathrm{k}}}{\Delta z^{2}}\right)+\left(1-\frac{i \Delta t V_{\mathrm{j}}^{\mathrm{k}}}{\hbar}\right) \Psi_{\mathrm{j}}^{\mathrm{k}} \\
& =\left(\frac{i \hbar \Delta t}{2 m \Delta z^{2}}\right) \Psi_{\mathrm{j}-1}^{\mathrm{k}}+\left(1-\frac{i \Delta t V_{\mathrm{j}}^{\mathrm{k}}}{\hbar}+\frac{i \hbar \Delta t}{m \Delta z^{2}}\right) \Psi_{\mathrm{j}}^{\mathrm{k}}+\left(\frac{i \hbar \Delta t}{2 m \Delta z^{2}}\right) \Psi_{\mathrm{j}+1}^{\mathrm{k}} .
\end{aligned}
$$

A função $\Psi_{\mathrm{j}}^{\mathrm{k}+1}$ torna-se então a base para o passo seguinte, e assim sucessivamente. É importante notar que (4.12) é uma equação matricial, pois $\Psi_{\mathrm{j}}^{\mathrm{k}+1}$ e $\Psi_{\mathrm{j}}^{\mathrm{k}}$ podem ser vistos como vetores relacionados por uma transformação linear dada por

$$
\left(\begin{array}{c}
\Psi_{0}^{\mathrm{k}+1} \\
\Psi_{1}^{\mathrm{k}+1} \\
\Psi_{2}^{\mathrm{k}+1} \\
\vdots \\
\Psi_{\mathrm{N}-3}^{\mathrm{k}+1} \\
\Psi_{\mathrm{N}-2}^{\mathrm{k}+1} \\
\Psi_{\mathrm{N}-1}^{\mathrm{k}+1}
\end{array}\right)=\left(\begin{array}{ccccccccc}
\beta_{0}^{\mathrm{k}} & \alpha_{0} & 0 & 0 & \ldots & 0 & 0 & 0 & 0 \\
\alpha_{1} & \beta_{1}^{\mathrm{k}} & \alpha_{1} & 0 & \ldots & 0 & 0 & 0 & 0 \\
0 & \alpha_{2} & \beta_{2}^{\mathrm{k}} & \alpha_{2} & \ldots & 0 & 0 & 0 & 0 \\
\vdots & \vdots & \vdots & \vdots & \ddots & \vdots & \vdots & \vdots & \\
0 & 0 & 0 & 0 & \ldots & \alpha_{\mathrm{N}-3} & \beta_{N-3}^{\mathrm{k}} & \alpha_{\mathrm{N}-3} & 0 \\
0 & 0 & 0 & 0 & \ldots & 0 & \alpha_{\mathrm{N}-2} & \beta_{N-2}^{\mathrm{k}} & \alpha_{\mathrm{N}-2} \\
0 & 0 & 0 & 0 & \ldots & 0 & 0 & \alpha_{\mathrm{N}-1} & \beta_{\mathrm{N}-1}^{\mathrm{k}}
\end{array}\right)\left(\begin{array}{c}
\Psi_{0}^{\mathrm{k}} \\
\Psi_{1}^{\mathrm{k}} \\
\Psi_{2}^{\mathrm{k}} \\
\vdots \\
\Psi_{\mathrm{N}-3}^{\mathrm{k}} \\
\Psi_{\mathrm{N}-2}^{\mathrm{k}} \\
\Psi_{\mathrm{N}-1}^{\mathrm{k}}
\end{array}\right)
$$

onde:

$$
\begin{aligned}
\alpha_{\mathrm{j}} & =\frac{i \hbar \Delta t}{2 m \Delta z^{2}} \\
\beta_{\mathrm{j}}^{\mathrm{k}} & =1-\frac{i \Delta t V_{\mathrm{j}}^{\mathrm{k}}}{\hbar}+\frac{i \hbar \Delta t}{m \Delta z^{2}}
\end{aligned}
$$

Nas subseções a seguir iremos explorar as escolhas que podemos fazer acerca do arranjo utilizado na Eq. (4.10), inclusive introduzindo algumas modificações para suportar a dependência espacial da massa efetiva dada pela Eq. (3.9).

\subsubsection{Métodos de Runge-Kutta}

Antes de introduzir os métodos de Runge-Kutta, é interessante apresentar o método de Euler, que é um caso particular dos métodos de Runge-Kutta, mas que facilita na compreensão do funcionamento desta classe de métodos. 
Se $\Psi(z, t)$ for uma função contínua e infinitamente derivável no intervalo $t \in\left[t_{0}, t_{\mathrm{f}}\right]$, a expansão de $\Psi(z, t)$ em torno do ponto $t_{0}$ para se obter a solução em $t=t_{0}+\Delta t$ pode ser expressa como [35].

$$
\Psi\left(z, t_{0}+\Delta t\right)=\sum_{\mathrm{n}=0}^{\infty} \frac{\Delta t^{\mathrm{n}}}{\mathrm{n} !}\left(\frac{\partial}{\partial t}\right)^{\mathrm{n}} \Psi\left(z, t_{0}\right) .
$$

O método de Euler consiste em truncar a série de Taylor da expressão (4.15) mantendo apenas os primeiros dois termos, isto é

$$
\Psi\left(z, t_{0}+\Delta t\right) \approx \Psi\left(z, t_{0}\right)+\Delta t f\left(z, t, \Psi\left(z, t_{0}, \Psi\left(z, t_{0}\right)\right)\right)
$$

onde,

$$
\frac{\partial}{\partial t} \Psi(z, t)=f(z, t, \Psi(z, t))
$$

Note que utilizando a Eq. (4.10) como aproximação para (4.17), temos um paralelo direto entre (4.16) e (4.13), ou seja, a aproximação das diferenças finitas descrita em (4.13) é totalmente equivalente a truncar a série de Taylor em (4.15) no segundo termo. A implementação computacional pode ser feita chamando $D$ a matriz na Eq. (4.13), P0 o vetor $\Psi(z, t), P 1$ o vetor $\Psi(z, t+\Delta t)$ e $d t$ o intervalo de tempo $\Delta t$. Com estas definições podemos escrever:

$1 \ldots$

$2 \mathrm{P} 1=\mathrm{P} 0+\mathrm{dt} * \mathrm{D} * \mathrm{P} 0$

$3 \ldots$

Assim como o método de Euler, os métodos de Runge-Kutta procuram estimar o valor de $\Psi(z, t+\Delta t)$ com base em $\Psi(z, t)$. Entretanto, o passo $\Psi(z, t+\Delta t)$ é calculado através de alguns passos intermediários. O método de Runge-Kutta de segunda ordem calcula uma aproximação para o tempo $t+\frac{\Delta t}{2}$ com base no tempo $t$ e a utiliza para melhor estimar o valor da função no tempo $t+\Delta t$. O método de Runge-Kutta de quarta ordem, apresentado a seguir, utiliza 4 aproximações intermediárias e incorre em um erro de truncamento de ordem $O\left(\Delta t^{4}\right)$.[35] Utilizando a notação semelhante à da expressão (4.17), definem-se as expressões a seguir [35]:

$$
\begin{gathered}
f_{0}=f\left(z, t_{0}, \Psi\left(z, t_{0}\right)\right) \\
f_{1}=f\left(z, t_{0}+\frac{\Delta t}{2}, \Psi\left(z, t_{0}\right)+\frac{\Delta t}{2} f_{0}\right) \\
f_{2}=f\left(z, t_{0}+\frac{\Delta t}{2}, \Psi\left(z, t_{0}\right)+\frac{\Delta t}{2} f_{1}\right)
\end{gathered}
$$




$$
f_{3}=f\left(z, t_{0}+\Delta t, \Psi\left(z, t_{0}\right)+\Delta t f_{2}\right)
$$

onde o passo $\Psi(z, t+\Delta t)$ é finalmente aproximado pela expressão,

$$
\Psi(z, t+\Delta t)=\Psi(z, t)+\frac{\Delta t}{6}\left(f_{0}+2 f_{1}+2 f_{2}+f_{3}\right)
$$

A implementação em um programa de computador pode ser feita como:

$1 \ldots$

$2 \mathrm{f} 0=\mathrm{D} *(\mathrm{P} 0)$

$3 \mathrm{f} 1=\mathrm{D} *(\mathrm{P} 0+\mathrm{dt} * \mathrm{f} 0 / 2)$

$4 \mathrm{f} 2=\mathrm{D} *(\mathrm{P} 0+\mathrm{dt} * \mathrm{f} 1 / 2)$

$5 \mathrm{f} 3=\mathrm{D} *(\mathrm{P} 0+\mathrm{dt} * \mathrm{f} 2)$

$6 \mathrm{P} 1=\mathrm{P} 0+\mathrm{dt} *(\mathrm{f} 0+2 * \mathrm{f} 1+2 * \mathrm{f} 2+\mathrm{f} 3) / 6$

$7 \ldots$

Este método é explícito, pois para calcular a função no tempo $t+\Delta t$ são necessários somente valores no tempo $t$, ou seja, apenas valores conhecido.[35]

\subsubsection{Método de Crank-Nicolson}

Neste método, é utilizada a aproximação por diferenças centrais com o lado esquerdo da Eq. (4.11) posicionado no momento $t+\frac{\Delta t}{2}$ e o lado direito como sendo a média dos momentos $t$ e $t+\Delta t$, ou seja:

$$
\begin{aligned}
\frac{\Psi_{\mathrm{j}}^{\mathrm{k}+1}-\Psi_{\mathrm{j}}^{\mathrm{k}}}{\Delta t}= & \frac{1}{2}\left[\frac{i \hbar}{2 m}\left(\frac{\Psi_{\mathrm{j}+1}^{\mathrm{k}+1}-2 \Psi_{\mathrm{j}}^{\mathrm{k}+1}-\Psi_{\mathrm{j}-1}^{\mathrm{k}+1}}{\Delta z^{2}}\right)-\frac{i V_{\mathrm{j}}^{\mathrm{k}+1}}{\hbar} \Psi_{\mathrm{j}}^{\mathrm{k}+1}\right]+ \\
& +\frac{1}{2}\left[\frac{i \hbar}{2 m}\left(\frac{\Psi_{\mathrm{j}+1}^{\mathrm{k}}-2 \Psi_{\mathrm{j}}^{\mathrm{k}}-\Psi_{\mathrm{j}-1}^{\mathrm{k}}}{\Delta z^{2}}\right)-\frac{i V_{\mathrm{j}}^{\mathrm{k}}}{\hbar} \Psi_{\mathrm{j}}^{\mathrm{k}}\right]
\end{aligned}
$$

que pode ser reescrita como

$$
\alpha_{\mathrm{j}}^{\prime} \Psi_{\mathrm{j}-1}^{\mathrm{k}+1}+\beta_{\mathrm{j}}^{\mathrm{k}+1^{\prime}} \Psi_{\mathrm{j}}^{\mathrm{k}+1}+\alpha_{\mathrm{j}}^{\prime} \Psi_{\mathrm{j}+1}^{\mathrm{k}+1} \approx-\alpha_{\mathrm{j}}^{\prime} \Psi_{\mathrm{j}-1}^{\mathrm{k}}+\beta_{\mathrm{j}}^{\mathrm{k} \prime \prime} \Psi_{\mathrm{j}}^{\mathrm{k}}-\alpha_{\mathrm{j}}^{\prime} \Psi_{\mathrm{j}+1}^{\mathrm{k}}
$$

$\mathrm{Ou}$

$$
B \Psi^{\mathrm{k}+1} \approx C \Psi^{\mathrm{k}} \rightarrow \Psi^{\mathrm{k}+1} \approx B^{-1} C \Psi^{\mathrm{k}}
$$

onde definimos

$$
\begin{aligned}
\alpha_{\mathrm{j}}^{\prime} & =-\frac{\Delta t \alpha_{\mathrm{j}}}{2} \\
\beta_{\mathrm{j}}^{\mathrm{k}^{\prime}} & =1-\frac{\Delta t \beta_{\mathrm{j}}^{\mathrm{k}}}{2} \\
\beta_{\mathrm{j}}^{\mathrm{k}^{\prime \prime}} & =1+\frac{\Delta t \beta_{\mathrm{j}}}{2}
\end{aligned}
$$




$$
\begin{gathered}
B=\left(\begin{array}{cccccccccc}
\beta_{0}^{\mathrm{k}+1^{\prime}} & \alpha_{0}^{\prime} & 0 & 0 & \ldots & 0 & 0 & 0 & 0 \\
\alpha_{1}^{\prime} & \beta_{1}^{\mathrm{k}+1^{\prime}} & \alpha_{1}^{\prime} & 0 & \ldots & 0 & 0 & 0 & 0 \\
0 & \alpha_{2}^{\prime} & \beta_{2}^{\mathrm{k}+1^{\prime}} & \alpha_{2}^{\prime} & \ldots & 0 & 0 & 0 & 0 \\
\vdots & \vdots & \vdots & \vdots & \ddots & \vdots & \vdots & \vdots & \\
0 & 0 & 0 & 0 & \ldots & \alpha_{\mathrm{N}-3}^{\prime} & \beta_{\mathrm{N}-3}^{\mathrm{k}+1^{\prime}} & \alpha_{\mathrm{N}-3}^{\prime} & 0 \\
0 & 0 & 0 & 0 & \ldots & 0 & \alpha_{\mathrm{N}-2}^{\prime} & \beta_{\mathrm{N}-2}^{\mathrm{k}+1^{\prime}} & \alpha_{\mathrm{N}-2}^{\prime} \\
0 & 0 & & 0 & 0 & \ldots & 0 & 0 & \alpha_{N-1}^{\prime} & \beta_{\mathrm{N}-1}^{\mathrm{k}+1^{\prime}}
\end{array}\right) \\
C=\left(\begin{array}{ccccccccc}
\beta_{0}^{\mathrm{k}^{\prime \prime}} & -\alpha_{0}^{\prime} & 0 & \ldots & 0 & 0 & 0 & \\
-\alpha_{1}^{\prime} & \beta_{1}^{\mathrm{k}^{\prime \prime}} & -\alpha_{1}^{\prime} & \ldots & 0 & 0 & 0 \\
0 & -\alpha_{2}^{\prime} & \beta_{2}^{\mathrm{k}^{\prime \prime}} & \ldots & 0 & 0 & 0 \\
\vdots & \vdots & \vdots & \ddots & \vdots & \vdots & \\
0 & 0 & 0 & \ldots & \beta_{\mathrm{N}-3}^{\mathrm{k}}{ }^{\prime \prime} & -\alpha_{\mathrm{N}-3}^{\prime} & 0 \\
0 & 0 & 0 & \ldots & -\alpha_{\mathrm{N}-2}^{\prime} & \beta_{\mathrm{N}-2}^{\mathrm{k}}{ }^{\prime \prime} & -\alpha_{\mathrm{N}-2}^{\prime} \\
0 & 0 & 0 & \ldots & 0 & -\alpha_{\mathrm{N}-1}^{\prime} & \beta_{\mathrm{N}-1}^{\mathrm{k}}{ }^{\prime \prime}
\end{array}\right)
\end{gathered}
$$

sendo $B^{-1}$ a matriz inversa de $B$. Chamando as matrizes $B$ (4.24), $C$ (4.25), $P 0$ o vetor $\Psi_{\mathrm{j}}^{\mathrm{k}}, P 1$ o vetor $\Psi_{\mathrm{j}}^{\mathrm{k}+1}$ e $d t$ o intervalo de tempo $\Delta t$, a implementação em um programa de computador pode ser feita como:

$1 \ldots$

$2 \mathrm{P} 1=\operatorname{inv}(\mathrm{B}) * \mathrm{C} * \mathrm{P} 0$

$3 \ldots$

Quando o potencial, e portanto o hamiltoniano, é independente do tempo, é necessário inverter a matriz $B$ somente uma vez, mas quando o potencial for dependente do tempo, a inversão será necessária a cada passo de tempo. Isto é algo que pode tornar o método extremamente lento. Em nossas aplicações, este foi um fator que inviabilizou a utilização desde método para fins de cálculo da fotocorrente, por exemplo.

Este método é implícito, pois para calcular a função no tempo $t+\Delta t$ são necessários valores no tempo $t+\frac{\Delta t}{2}$, que são obtidos de forma implícita.[35]

\subsubsection{Estabilidade}

O conceito de estabilidade para um método numérico diz respeito à forma como o erro de truncamento se comporta na medida em que o passo diminui, ou seja, se o erro de truncamento diminui conforme $\Delta t \rightarrow 0$ e $\Delta x \rightarrow 0$, o método é estável. Caso contrário, ele é dito instável [35]. No caso de equações parabólicas, o método de Crank-Nicolson é estável para passos $\Delta z$ e $\Delta t$ que obedeçam a expressão (4.26). A mesma condição é valida 
para o método de Runge-Kutta.[35]

$$
0<\frac{\Delta t}{(\Delta z)^{2}} \leq \frac{1}{2}
$$

\subsubsection{Massa Efetiva com Dependência Espacial}

Como já vimos nos capítulos anteriores, os poços quânticos de heteroestruturas semicondutoras são formados por materiais diferentes nas barreiras e no poço. Isto faz com que a massa efetiva também seja diferente nas barreiras e no poço. A forma de endereçar este problema é através da Eq. (3.9). Vamos aqui apenas descrever o arranjo utilizando diferenças finitas que será utilizado sempre que necessário.

A Eq. (3.9) pode ser escrita em uma dimensão como sendo

$$
-\frac{\hbar^{2}}{2} \frac{\partial}{\partial z} \frac{1}{m(z)} \frac{\partial}{\partial z} \Psi(z, t)+V(z) \Psi(z, t)=i \hbar \frac{\partial}{\partial t} \Psi(z, t) .
$$

Vamos aqui adotar o esquema das diferenças finitas centrais aplicado duas vezes. Também vamos adotar as seguintes interpolações:

$$
\begin{aligned}
\Psi(z+\Delta z / 2, t) & =\Psi_{\mathrm{j}+1 / 2}^{\mathrm{k}}=\frac{\Psi_{\mathrm{j}+1}^{\mathrm{k}}-\Psi_{\mathrm{j}}^{\mathrm{k}}}{\Delta z} \\
m(z+\Delta z / 2) & =m_{\mathrm{j}+1 / 2}=\frac{m_{\mathrm{j}+1}-m_{\mathrm{j}}}{\Delta z} .
\end{aligned}
$$

A primeira derivada parcial de $\Psi(z, t)$ multiplicada pela massa fica então:

$$
\frac{1}{m(z)} \frac{\partial}{\partial z} \Psi(z, t) \approx \frac{1}{m_{\mathrm{j}}} \frac{\Psi_{\mathrm{j}+1 / 2}^{k}-\Psi_{\mathrm{j}-1 / 2}^{k}}{\Delta z},
$$

de modo que a segunda derivada resulta em:

$$
\begin{aligned}
\frac{\partial}{\partial z} \frac{1}{m(z)} \frac{\partial}{\partial z} \Psi(z, t) & \approx \frac{1}{\Delta z}\left[\frac{1}{m_{\mathrm{j}+1 / 2}} \frac{\Psi_{\mathrm{j}+1}^{k}-\Psi_{\mathrm{j}}^{\mathrm{k}}}{\Delta z}-\frac{1}{m_{\mathrm{j}-1 / 2}} \frac{\Psi_{\mathrm{j}}^{\mathrm{k}}-\Psi_{\mathrm{j}-1}^{\mathrm{k}}}{\Delta z}\right] \\
& \approx \frac{1}{\Delta z^{2}}\left[\frac{\Psi_{\mathrm{j}+1}^{\mathrm{k}}-\Psi_{\mathrm{j}}^{\mathrm{k}}}{m_{\mathrm{j}+1 / 2}}-\frac{\Psi_{\mathrm{j}}^{\mathrm{k}}-\Psi_{\mathrm{j}-1}^{\mathrm{k}}}{m_{\mathrm{j}-1 / 2}}\right] .
\end{aligned}
$$

A ação do operador hamiltoniano sobre um estado pode então ser representada por

$$
\mathbf{H}^{\mathrm{k}} \Psi_{\mathrm{j}}^{\mathrm{k}}=-\frac{\hbar^{2}}{2 \Delta z^{2}}\left[\frac{\Psi_{\mathrm{j}+1}^{\mathrm{k}}-\Psi_{\mathrm{j}}^{\mathrm{k}}}{m_{\mathrm{j}+1 / 2}}-\frac{\Psi_{\mathrm{j}}^{\mathrm{k}}-\Psi_{\mathrm{j}-1}^{\mathrm{k}}}{m_{\mathrm{j}-1 / 2}}\right]+V_{\mathrm{j}}^{\mathrm{k}} \Psi_{\mathrm{j}}^{\mathrm{k}},
$$

que equivale a multiplicação de uma matriz por um vetor, como na Eq. (4.13). Veja que, em princípio, isto é válido para um hamiltoniano com dependência temporal.

A Eq. (4.31) pode ser usada em conjunto com as Eqs. (4.12), (4.19) e (4.22) para obter a evolução temporal de um estado qualquer. 


\subsection{O Método Pseudo-Espectral}

Este método assume que a solução formal da Eq. (2.14) é dada pela função:

$$
\Psi(z, t)=e^{-i \mathbf{H}\left(t-t_{0}\right) / \hbar} \Psi\left(z, t_{0}\right)=e^{-i(\mathbf{T}+\mathbf{V}) \delta_{t} / \hbar} \Psi\left(z, t_{0}\right),
$$

onde definimos $\delta_{\mathrm{t}}=t-t_{0}$. Formalmente, a Eq. (4.32) fornece uma prescrição para o cálculo de $\Psi(z, t)$ em qualquer instante $t$ uma vez conhecida a função $\Psi\left(z, t_{0}\right)$ no instante $t_{0}$, para isso utilizando o operador propagação temporal

$$
\mathbf{U}=e^{-i \mathbf{H}\left(t-t_{0}\right) / \hbar}=e^{-i(\mathbf{T}+\mathbf{V}) \delta_{\mathrm{t}} / \hbar} .
$$

Em um primeiro momento, é extremamente tentador escrevermos que a exponencial da soma dos operadores possa ser escrita como sendo o produto de duas exponenciais:

$$
e^{-i(\mathbf{T}+\mathbf{V}) \delta_{\mathrm{t}} / \hbar}=e^{-i \mathbf{T} \delta_{\mathrm{t}} / \hbar} e^{-i \mathbf{V} \delta_{\mathrm{t}} / \hbar} .
$$

Entretanto, a igualdade na expressão (4.34) não é verdadeira, pois o teorema de BakerCampbell-Hausdorff [36] estabelece que

$$
\exp (\mathbf{A}) \exp (\mathbf{B})=\exp (\mathbf{C})
$$

se, e apenas se,

$$
\mathbf{C}=\mathbf{A}+\mathbf{B}+\frac{1}{2}[\mathbf{A}, \mathbf{B}]+\frac{1}{12}([\mathbf{A},[\mathbf{A}, \mathbf{B}]]+[[\mathbf{A}, \mathbf{B}], \mathbf{B}])+\ldots
$$

onde $[\mathbf{A}, \mathbf{B}]=\mathbf{A B}-\mathbf{B A}$ é o comutador entre os operadores $\mathbf{A}$ e $\mathbf{B}$. Ou seja, a expressão $e^{\mathbf{A}} e^{\mathbf{B}}=e^{\mathbf{A}+\mathbf{B}}$ é válida apenas se, e somente se, os operadores $\mathbf{A}$ e $\mathbf{B}$ comutam, isto é, $[\mathbf{A}, \mathbf{B}]=\mathbf{A B}-\mathbf{B A}=0$. E este não é o caso dos operadores $\mathbf{T}$ e $\mathbf{V}$.

Se a expressão (4.35) fosse válida então teríamos conseguido dividir o operador evolução temporal em dois termos, e cada fator poderia ser tratado separadamente. Como veremos em seguida, embora essa igualdade não seja válida, a decomposição será útil para o desenvolvimento de uma aproximação de erro conhecido mesmo quando os operadores A e B não comutam.

Para escrever uma expressão aproximada da expressão (4.32) em termos do produto entre exponenciais, usaremos o procedimento de Feit et al.[1, 2, 37] segundo o qual a exponencial $\exp (\mathbf{A}+\mathbf{B})$ é escrita em termos das exponenciais dos operadores $\exp (\mathbf{A} / 2)$ $\mathrm{e} \exp (\mathbf{B})$ como

$$
\exp (\mathbf{A}+\mathbf{B}) \approx \exp (\mathbf{A} / 2) \exp (\mathbf{B}) \exp (\mathbf{A} / 2)
$$

onde a diferença entre os dois termos da relação acima permite avaliar o erro cometido na aproximação. Utilizando a definição de exponencial do operador $\mathbf{A}$, que é dada como a série infinita

$$
\exp (\mathbf{A})=e^{\mathbf{A}}=\sum_{\mathrm{n}=0}^{\infty} \frac{\mathbf{A}^{\mathrm{n}}}{\mathrm{n} !}=\mathbf{1}+\mathbf{A}+\frac{\mathbf{A}^{2}}{2 !}+\ldots
$$


e subtraindo a expressão

$$
\begin{aligned}
e^{\mathbf{A} / 2} e^{\mathbf{B}} e^{\mathbf{A} / 2}= & {\left[\mathbf{1}+\left(\frac{\mathbf{A}}{2}\right)+\frac{1}{2 !}\left(\frac{\mathbf{A}}{2}\right)^{2}+\ldots\right]\left[\mathbf{1}+\mathbf{B}+\frac{1}{2 !} \mathbf{B}^{2}+\ldots\right] } \\
& {\left[\mathbf{1}+\left(\frac{\mathbf{A}}{2}\right)+\frac{1}{2 !}\left(\frac{\mathbf{A}}{2}\right)^{2}+\ldots\right] }
\end{aligned}
$$

de

$$
e^{(\mathbf{A}+\mathbf{B})}=\mathbf{1}+(\mathbf{A}+\mathbf{B})+\frac{1}{2 !}(\mathbf{A}+\mathbf{B})^{2}+\frac{1}{3 !}(\mathbf{A}+\mathbf{B})^{3}+\ldots
$$

obtém-se

$$
e^{(\mathbf{A}+\mathbf{B})}=e^{\mathbf{A} / 2} e^{\mathbf{B}} e^{\mathbf{A} / 2}+O\left(\delta_{t}^{3}\right) .
$$

com erro $O$ da ordem de $\delta_{t}^{3}$. Definindo os operadores A e $\mathbf{B}$ como

$$
\mathbf{A}=-i \mathbf{V} \frac{\delta_{\mathrm{t}}}{\hbar}
$$

e

$$
\mathbf{B}=-i \mathbf{T} \frac{\delta_{\mathrm{t}}}{\hbar}
$$

a exponencial da expressão (4.33) pode ser escrita como

$$
e^{-i(\mathbf{T}+\mathbf{V}) \delta_{\mathrm{t}} / \hbar} \approx e^{-i \mathbf{V}\left(\delta_{\mathrm{t}}\right) / 2 \hbar} e^{-i \mathbf{T}\left(\delta_{\mathrm{t}}\right) / \hbar} e^{-i \mathbf{V}\left(\delta_{\mathrm{t}}\right) / 2 \hbar}
$$

a menos de um erro da ordem de $O\left(\delta_{\mathrm{t}}^{3}\right)$. Assim, a expressão da função de onda (4.32) pode finalmente ser escrita de forma aproximada como: ${ }^{1}$

$$
\Psi(z, t) \approx e^{-i \mathbf{V}\left(\delta_{\mathrm{t}}\right) / 2 \hbar} e^{-i \mathbf{T}\left(\delta_{\mathrm{t}}\right) / \hbar} e^{-i \mathbf{V}\left(\delta_{\mathrm{t}}\right) / 2 \hbar} \Psi\left(z, t_{0}\right)
$$

Essa técnica de se expandir a exponencial de um operador em várias exponenciais é chamada de Split Operator Method.[1, 2, 37]. A Eq. (4.44) pode ser iterada permitindo, desta forma, acompanhar a evolução da função de onda de acordo com o potencial a que estiver sendo submetida. É obvio que, em vez da divisão em duas partes iguais do operador energia potencial, como feito em (4.37), poder-se-ia ter feito uma divisão em partes iguais do operador energia cinética, sem prejuízo de acurácia. Podemos escolher uma destas duas partições, ou mesmo outras, dependendo da implementação do programa computacional utilizado para a propagação temporal da função de onda.

$\overline{1}$ Observe que cada vez que se expande a exponencial da expressão (4.32) adiciona-se um erro da ordem de $\delta_{\mathrm{t}}^{\mathrm{n}}$. Por exemplo, a separação

$$
e^{-i(\mathbf{T}+\mathbf{V}) \delta_{\mathrm{t}} / \hbar} \approx e^{-i \mathbf{T} \delta_{\mathrm{t}} / \hbar} e^{-i \mathbf{V}\left(\delta_{\mathrm{t}}\right) / \hbar}+O\left(\delta_{\mathrm{t}}^{2}\right)
$$

ocasionará um erro da ordem de $\delta_{\mathrm{t}}^{2}$ na aproximação. 


\subsubsection{Etapas para Evolução Temporal}

Tecnicamente, para realizar os cálculos numéricos é necessário a implementação das seguintes etapas:

- A primeira, diz respeito à representação da função de onda e dos operadores em um conjunto discreto finito de pontos no espaço (malha), que pode ser uniforme ou não (geralmente a malha uniforme não acarreta erros maiores);

- A segunda consiste no processo de propagação da função de onda no tempo, propriamente dito.

Existe uma variedade de diferentes abordagens para se obter os sucessivos valores de $\Psi(z, t)$ utilizando-se a Eq. (4.44). Os principais critérios para a escolha do método são as propriedades de escala, as questões de precisão e convergência, e a conservação da norma durante a propagação, que também deve ser levada em consideração. Grosso modo, pode-se realizar o processo de propagação em 3 etapas:

1. Calcula-se

$$
\Phi_{1}\left(z, t_{0}\right)=e^{-i \mathbf{V} \delta_{t} / 2 \hbar} \Psi\left(z, t_{0}\right)
$$

sendo $\Psi\left(z, t_{0}\right)$ a condição inicial.

2. Utilizando o resultado anterior, calcula-se:

$$
\Phi_{2}\left(z, t_{0}\right)=e^{-i \mathbf{T} \delta_{\mathrm{t}} / \hbar} \Phi_{1}\left(z, t_{0}\right)
$$

3. Finalmente, a função de onda para um instante $t_{1}=t_{0}+\delta_{\mathrm{t}}$ é obtida por

$$
\Psi\left(z, t_{1}\right)=e^{-i \mathbf{V} \delta_{t} / 2 \hbar} \Phi_{2}\left(z, t_{0}\right)
$$

No cálculo de $\Psi\left(z, t_{2}\right)$ utiliza-se $\Psi\left(z, t_{1}\right)$ como condição inicial e assim por diante. Para a ESIT, o operador V é, simplesmente, uma função de $z$ e o cálculo da exponencial desse operador na primeira etapa é trivial. A dificuldade para a implementação prática do processo de propagação encontra-se no cálculo do fator $\exp \left(-i \mathbf{T} \delta_{\mathrm{t}} / \hbar\right)$, já que ele apresenta uma derivada de segunda ordem em relação a $z$, de modo que a segunda etapa do procedimento requer alguma técnica para o cálculo da exponencial. Esta dificuldade no processo de propagação de pacotes de onda foi contornada quando a técnica de Transformada Rápida de Fourier (FFT, sigla do inglês Fast Fourier Transform) foi aplicada por Feit e Fleck [1, 2] e Kosloff e Kosloff [3] para a avaliação da parte da energia cinética na hamiltoniana. A essência do método é o fato que o operador momento quântico, não local na representação de coordenadas, é local na representação dos momentos, onde sua ação pode ser avaliada por simples multiplicação. 
Segundo esta estratégia, para a realização da segunda etapa deve-se primeiro obter $\Phi_{1}(k)$ que é a transformada de Fourier de $\Phi_{1}(x)$,

$$
\Phi_{1}(k)=\mathcal{F}\left[\Phi_{1}(z)\right]=\frac{1}{\sqrt{2 \pi}} \int_{-\infty}^{\infty} \Phi_{1}(z) e^{-i k z} d z
$$

e também a relação inversa:

$$
\Phi(z)=\mathcal{F}^{-1}\left[\Phi_{1}(k)\right]=\frac{1}{\sqrt{2 \pi}} \int_{-\infty}^{\infty} \Phi_{1}(k) e^{+i k z} d k
$$

Assim procedendo, teremos:

$$
\begin{aligned}
e^{-i \mathbf{T} \delta_{\mathrm{t}} / \hbar} \Phi(z) & =e^{-i \mathbf{T} \delta_{\mathrm{t}} / \hbar} \frac{1}{\sqrt{2 \pi}} \int_{-\infty}^{\infty} \Phi_{1}(k) e^{+i k z} d k \\
& =\left[1+\frac{-i \mathbf{T} \delta_{\mathrm{t}}}{\hbar}+\frac{(-i)^{2} \mathbf{T} \mathbf{T} \delta_{\mathrm{t}}^{2}}{2 ! \hbar^{2}}+\ldots\right] \frac{1}{\sqrt{2 \pi}} \int_{-\infty}^{\infty} \Phi_{1}(k) e^{+i k z} d k \\
& =\frac{1}{\sqrt{2 \pi}} \int_{-\infty}^{\infty} \Phi_{1}(k)\left[1+\frac{-i \mathbf{T} \delta_{\mathrm{t}}}{\hbar}+\frac{(-i)^{2} \mathbf{T} \mathbf{T} \delta_{\mathrm{t}}^{2}}{2 ! \hbar^{2}}+\ldots\right] e^{+i k z} d k \\
& =\frac{1}{\sqrt{2 \pi}} \int_{-\infty}^{\infty} \Phi_{1}(k)\left[1+\frac{-i}{\hbar}\left(\frac{-\hbar^{2} \delta_{\mathrm{t}}}{2 m}\right) \frac{d^{2}}{d z^{2}}+\frac{1}{2 !}\left(\frac{-i-\hbar^{2} \delta_{\mathrm{t}}}{\hbar}\right)^{2} \frac{d^{4}}{d z^{4}}+\ldots\right] e^{+i k z} d k \\
& =\frac{1}{\sqrt{2 \pi}} \int_{-\infty}^{\infty} \Phi_{1}(k)\left[1+\frac{-i T(k) \delta_{\mathrm{t}}}{\hbar}+\frac{1}{2 !}\left(\frac{-i T(k) \delta_{\mathrm{t}}}{\hbar}\right)+\ldots\right] e^{+i k z} d k \\
& =\frac{1}{\sqrt{2 \pi}} \int_{-\infty}^{\infty} \Phi_{1}(k)\left[e^{-i T(k) \delta_{\mathrm{t}} / \hbar}\right] e^{+i k z} d k \\
& =\frac{1}{\sqrt{2 \pi}} \int_{-\infty}^{\infty}\left[e^{-i T(k) \delta_{\mathrm{t}} / \hbar}\right] \Phi_{1}(k) e^{+i k z} d k \\
& =\mathcal{F}\left[e^{-i T(k) \delta_{\mathrm{t}} / \hbar}\right]
\end{aligned}
$$

onde introduzimos o termo da energia cinética $T(k)=\frac{\hbar^{2} k^{2}}{2 m}$. Portanto, a ideia básica do método espectral pode ser resumida como mostrado na Tabela 1:

\subsubsection{Discretização do Espaço e Função de Onda Inicial}

Para implementar o algoritmo da evolução temporal da função de onda é necessário definir a região do espaço, de comprimento $L_{0}$ no caso unidimensional, onde a função será evoluída. Em princípio, essa região precisa cobrir todo o espaço da função de onda inicial ou, no mínimo, que suas extremidades têm que ser tais que possam ser consideradas nulas. Escolhido o comprimento $L_{0}$ do intervalo, é necessário definir uma grade de pontos no espaço onde a função será calculada. Para que seja possível utilizar a FFT, é importante que o número $N$ de pontos nesta grade seja da forma $N=2^{n}$ ( $n$ inteiro positivo).

A função de onda inicial $\Psi(z, t=0)=\Psi(z, 0)$ é frequentemente definida analiticamente, isto é, utiliza-se funções analíticas bem conhecidas. Em geral, e neste trabalho, 
Tabela 1: Etapas para evolução temporal segundo o método espectral.

\begin{tabular}{|c|c|}
\hline Operação & Simbologia \\
\hline 1. Escolha de uma função inicial conveniente & $\Psi\left(z, t_{0}\right)$ \\
\hline $\begin{array}{l}\text { 2. Produto da função com o termo exponencial } \\
\text { envolvendo metade do potencial }\end{array}$ & $e^{-i \overline{\mathbf{V}} \bar{\delta}_{\mathrm{t}} / 2 \hbar} \Psi\left(z, t_{0}\right) \rightarrow \xi\left(z, t_{0}\right)$ \\
\hline 3. Transformada de Fourier sobre o resultado & $\mathcal{F}\left[\xi\left(z, t_{0}\right)\right] \rightarrow \tilde{\xi}\left(k, t_{0}\right)$ \\
\hline $\begin{array}{l}\text { 4. Produto da função no espaço recíproco } \\
\text { com o termo de energia cinética }\end{array}$ & $e^{-i \mathbf{T} \delta_{\mathrm{t}} / \hbar} \tilde{\xi}\left(k, t_{0}\right) \rightarrow \tilde{\eta}\left(k, t_{0}\right)$ \\
\hline 5. Transformada Inversa de Fourier & $\mathcal{F}^{-1}\left[\tilde{\eta}\left(k, t_{0}\right)\right] \rightarrow \eta\left(z, t_{0}\right)$ \\
\hline $\begin{array}{l}\text { 6. Produto da função com o termo } \\
\text { de metade do potencial }\end{array}$ & $e^{-i \mathbf{\mathbf { V }} \delta_{\mathrm{t}} / 2 \hbar} \eta\left(z, t_{0}\right) \rightarrow \Psi\left(z, t_{0}+\bar{\delta}_{\mathrm{t}}\right)$ \\
\hline
\end{tabular}

utilizamos um pacote de ondas com a forma de uma função gaussiana:

$$
\Psi(z, 0)=C e^{-\beta\left(z-z_{0}\right)^{2}}
$$

onde $C$ é a constante de normalização, $\beta$ e $z_{0}$ são parâmetros que definem a largura e a posição do máximo da gaussiana, respectivamente. Depois que a função de onda inicial e/ou operadores são definidos de forma analítica, a discretização é trivial.

A grade de pontos utilizada na discretização do espaço não precisa necessariamente ser equidistante. De fato, alguns métodos funcionam com redes não uniformes, ganhando vantagens de uma melhor amostragem da função de onda nas regiões de interesse. No entanto, a maioria dos métodos de propagação opera em grades equidistantes, com critérios de amostragem que variam muito de um método para outro. Tais redes equidistantes são definidas da seguinte forma:

$$
z_{\mathrm{n}}=\frac{\mathrm{n} L_{0}}{N}, \mathrm{n}=0, \ldots, N-1
$$

onde o índice $n$ refere-se a cada ponto de uma malha com $N$ pontos.

\subsubsection{Transformada Discreta de Fourier}

Como dito, o termo exponencial da energia cinética representa um problema no cálculo da função de onda. Para solucioná-lo, primeiramente, podemos calcular a Transformada de Fourier da função de onda definida na Eq. (4.46). Para fins computacionais a Transformada de Fourier deverá ser feita em uma função de onda representada em uma malha de pontos. Nesse ambiente, a integral da transformada precisa ser representada por um somatório e a Transformada de Fourier passa a ser chamada de Transformada Discreta de Fourier (Discrete Fourier Transform, DFT) [36]. 
Para definir a Transformada Discreta de Fourier que levará $f(z)$ em $g(k)$, é preciso definir no espaço de $z$ um intervalo de comprimento $L_{0}$ com $N$ pontos equidistantes. No espaço de $k$, existirão também $N$ pontos, dados por:

$$
k_{\mathrm{m}}=\frac{2 \pi \mathrm{m}}{L_{0}}, \mathrm{~m}=0, \ldots, N-1 .
$$

Com as definições das malhas dadas em (4.52) e (4.53), a Transformada Discreta de Fourier, $g\left(k_{\mathrm{m}}\right)$, será dada como

$$
g\left(k_{\mathrm{m}}\right)=\sum_{\mathrm{n}=0}^{N-1} f\left(z_{\mathrm{n}}\right) e^{-i \frac{2 \pi}{N} \mathrm{~nm}}, \mathrm{~m}=0, \ldots, N-1,
$$

e a transformada inversa será

$$
f\left(z_{\mathrm{n}}\right)=\frac{1}{N} \sum_{\mathrm{m}=0}^{N-1} g\left(k_{\mathrm{m}}\right) e^{+i \frac{2 \pi}{N} \mathrm{~nm}}, \mathrm{n}=0, \ldots, N-1 .
$$

Como é possível ver, a DFT transforma $N$ pontos de $f(z)$ em $N$ pontos de $g(k)$. Esses $N$ pontos do espaço $z$ correspondem a um intervalo $\left[z_{0}, z_{\mathrm{N}-1}\right]$ de comprimento $L_{0}=z_{N-1}-z_{0}$, igualmente espaçados por $\Delta z=\frac{L_{0}}{N}$. Isso deverá corresponder a um mesmo número $N$ de pontos no espaço de $k$, correspondendo a um intervalo $\left[k_{0}, k_{\mathrm{N}-1}\right]$, também igualmente espaçados por $\Delta k=\frac{k_{\mathrm{N}-1}-k_{0}}{N}$.

A DFT permite calcular a Transformada de Fourier com a precisão desejada. Porém, o esforço computacional envolvido é enorme. Cada ponto $g\left(k_{\mathrm{m}}\right)$ é gerado ao custo de $N$ repetições. Para gerar os $N$ pontos serão necessários $N^{2}$ iterações. Isso vai ficando inviável à medida que a precisão necessita ser aumentada.

Processadores modernos, comuns em computadores pessoais, possuem uma frequência de clock da ordem de $10^{9} \mathrm{~Hz}$. Supondo-se que cada iteração custe algumas dezenas de ciclos de máquina, para $N=2^{12}=4096$, seria necessário um tempo $\tau \approx \frac{4096^{2} \times 10}{10^{9}} \approx 0,17 \mathrm{~s}$ para realizar a transformada. Muito provavelmente seria necessário calcular a transformada inversa, o que já siginifica $\tau \approx 0,35 \mathrm{~s}$. Isso é um tempo relativamente alto em termos computacionais. Se para computadores modernos esse tempo é elevado, para computadores da década de 1960 quando a frequência de clock não chegava a $10^{6} \mathrm{~Hz}$, o cálculo do parágrafo acima levaria $\tau \approx 335 \mathrm{~s}$.

Nessa época, uma artigo de Cooley e colaboradores [38, 39] apresentou à comunidade científica o que ficou conhecido como Transformada Rápida de Fourier. Foi um tremendo ganho em termos computacionais, pois reduziu a complexidade da transformada de $N^{2}$ para $N / 2 \log _{2}^{N}$. Isso fez com que o cálculo das transformadas dadas nas Eqs. (4.54) e (4.55) que levavam $\tau \approx 335 \mathrm{~s}$ fosse realizado em $\tau \approx 0,49 \mathrm{~s}$.

A FFT surge a partir de uma rearranjo nos termos da DFT:

$$
g\left(k_{\mathrm{m}}\right)=\sum_{\mathrm{n}=0}^{N-1} f\left(z_{\mathrm{n}}\right) e^{-i \frac{2 \pi}{N} n \mathrm{~m}}=\sum_{\mathrm{n}=0}^{N / 2-1} f\left(z_{2 \mathrm{n}}\right) e^{-i \frac{2 \pi}{N}(2 \mathrm{n}) \mathrm{m}}+\sum_{\mathrm{n}=0}^{N / 2-1} f\left(z_{2 \mathrm{n}+1}\right) e^{-i \frac{2 \pi}{N}(2 \mathrm{n}+1) \mathrm{m}}
$$




\subsection{Cálculo de Autovalores e Autovetores}

Encontrar a função de onda do estado fundamental de um sistema quântico diretamente a partir de seu hamiltoniano é um processo complicado, sendo que apenas alguns sistemas conhecidos possuem soluções exatas. Nesta seção apresentamos duas abordagens, sendo a primeira conhecida como Propagação no Tempo Imaginário (Imaginary Time Propagation, ITP), que pode ser utilizada com os métodos descritos nas seções anteriores e a segunda sendo o Método da Iteração Inversa, que é um método específico para cálculo de autovalores e autovetores.

\subsubsection{Evolução em Tempo Imaginário: Função de Onda do Estado Funda- mental}

A equação de Schrödinger permite um procedimento matemático segundo o qual é possível obter o estado fundamental da função de onda com uma precisão arbitrária, conhecido como Propagação em Tempo Imaginário, que envolve a substituição do tempo imaginário em vez do tempo real na equação de Schrödinger dependente do tempo. Isto conduz à derivação de um operador em tempo imaginário que resulta no decaimento exponencial de todos os estados, exceto do estado fundamental, que pode ser então aplicado a qualquer função de onda de teste, inicial, para calcular uma aproximação para o estado fundamental real.

Vamos mostrar agora como a evolução em tempo imaginário leva ao estado fundamental. Para isso, considere uma hamiltoniana independente do tempo $\mathbf{H}$ com autoestados $\Phi_{\mathrm{n}}(z)$ e autoenergias dadas por $E_{\mathrm{n}}$, ordenadas em ordem crescente de energia como $E_{0}<E_{1}<E_{2}<\ldots E_{\mathrm{n}} \ldots$ Para cada autovalor há também uma função de onda correspondente, que é uma solução da equação de Schrödinger para o potencial $V(z)$. Para o n-ésimo autovalor, a função de onda

$$
\Phi_{\mathrm{n}}(z, t)=\Phi_{\mathrm{n}}(z) e^{-i E_{\mathrm{n}} t / \hbar}
$$

é uma solução particular da equação de Schrödinger para o potencial $V(z)$, que tem como solução geral a função:

$$
\Psi_{\mathrm{n}}(z, t)=\sum_{\mathrm{n}=0}^{\infty} c_{\mathrm{n}} \Phi_{\mathrm{n}}(z) e^{-i E_{\mathrm{n}} t / \hbar}
$$

Substituindo $t$ por $i \tau$ na solução geral e normalizando-a obtemos

$$
\Psi(z, t)=\frac{\sum_{\mathrm{n}} c_{\mathrm{n}} \Phi_{\mathrm{n}}(z) \exp \left(-\frac{E_{\mathrm{n}} \tau}{\hbar}\right)}{\sqrt{\sum_{\mathrm{n}}\left|c_{\mathrm{n}}\right|^{2} \exp \left(-2 \frac{E_{\mathrm{n}} \tau}{\hbar}\right)}}
$$


que ainda pode ser reescrita como

$$
\Psi(z, t)=\frac{c_{0} \Phi_{0}(z)+\sum_{\mathrm{n}=1}^{\infty} c_{\mathrm{n}} \Phi_{\mathrm{n}}(z) \exp \left(-\frac{E_{\mathrm{n}} \tau}{\hbar}\right)}{\sqrt{\sum_{\mathrm{n}=1}^{\infty}\left|c_{\mathrm{n}}\right|^{2} \exp \left(-2 \frac{E_{\mathrm{n}} \tau}{\hbar}\right)}}=\frac{\Phi_{0}(z)+\sum_{\mathrm{n}=1}^{\infty} \frac{c_{\mathrm{n}}}{c_{0}} \Phi_{\mathrm{n}}(z) \exp \left(\frac{\left(E_{0}-E_{\mathrm{n}}\right) \tau}{\hbar}\right)}{\sqrt{1+\sum_{\mathrm{n}=1}^{\infty}\left|\frac{c_{\mathrm{n}}}{c_{0}}\right|^{2} \exp \left(2 \frac{\left(E_{0}-E_{\mathrm{n}}\right) \tau}{\hbar}\right)}}
$$

Sendo $E_{0}$ a menor de todas as energias, as exponenciais tanto do numerador quanto do denominador da Eq. (4.60) tendem a zero quando $\tau \rightarrow \infty$, isso porque $E_{0}-E_{\mathrm{n}}<0$ para qualquer $n \geq 1$. Isso significa que:

$$
\lim _{\tau \rightarrow \infty} \Psi(z, t)=\Phi_{0}
$$

ou seja, a evolução temporal de $\Psi(z, t)$ em tempo imaginário $\tau$ até um tempo suficientemente grande $(\tau \rightarrow \infty)$ convergirá para sua autofunção do estado fundamental, $\Phi_{0}(z)$.

Os estados excitados são obtidos através do método da ortogonalização de GramSchmidt, bastante comum em álgebra linear para a obtenção de uma base ortogonal a partir de um conjunto de vetores não ortogonais inicialmente.[6, 24]

Seja $\left\{\Phi_{\mathrm{n}}(z, t)\right\}$ um conjunto de estados linearmente independentes, então assumese que o autoestado fundamental é $\Psi_{0}(z, t)=\Phi_{0}(z, t)$, sendo os demais autoestados obtidos através de:

$$
\begin{aligned}
\Psi_{0}(z, t) & =\Phi_{0}(z, t) \\
\Psi_{1}(z, t) & =\Phi_{1}(z, t)-\left\langle\Psi_{0}(z, t) \mid \Phi_{1}(z, t)\right\rangle, \Phi_{1}(z, t) \\
\Psi_{2}(z, t) & =\Phi_{2}(z, t)-\left\langle\Psi_{0}(z, t) \mid \Phi_{2}(z, t)\right\rangle \Phi_{2}(z, t)-\left\langle\Psi_{1}(z, t) \mid \Phi_{2}(z, t)\right\rangle \Phi_{2}(z, t) \\
\vdots & \\
\Psi_{\mathrm{n}}(z, t) & =\Phi_{\mathrm{n}}(z, t)-\sum_{\mathrm{i}=0}^{\mathrm{n}-1}\left\langle\Psi_{\mathrm{i}}(z, t) \mid \Phi_{\mathrm{n}}(z, t)\right\rangle \Phi_{\mathrm{i}}(z, t)
\end{aligned}
$$

\subsubsection{O Método da Iteração Inversa}

O método da Iteração Inversa possui uma premissa semelhante à ideia de evolução em tempo imaginário, ou seja, um estado qualquer $\phi(z)$ pode ser escrito como uma combinação linear dos autovetores $\psi_{n}(z)$ do operador hamiltoniano $\mathbf{H}$ independente do tempo como:

$$
\phi(z)=\sum_{\mathrm{n}} a_{\mathrm{n}} \psi_{\mathrm{n}}(z)
$$

A partir disso, utilizamos a Eq. (2.9) juntamente com o fato de que $\mathbf{H} \psi_{\mathrm{n}}(z)=E_{\mathrm{n}} \psi_{\mathrm{n}}(z)$ para podermos escrever

$$
\left(\frac{1}{\mathbf{H}-\epsilon}\right) \phi(z)=\left(\frac{1}{\mathbf{H}-\epsilon}\right) \sum_{\mathrm{n}} a_{\mathrm{n}} \psi_{\mathrm{n}}(z)=\sum_{\mathrm{n}} a_{\mathrm{n}}\left(\frac{1}{E_{\mathrm{n}}-\epsilon}\right) \psi_{\mathrm{n}}(z),
$$


onde $\epsilon$ é um número real. Quando $\epsilon$ é próximo a $E_{\mathrm{j}}$, a contribuição de $\psi_{\mathrm{j}}(z)$ em (4.63) será muito mais importante do que as demais, de modo que sucessivas aplicações de $(\mathbf{H}-\epsilon)^{-1}$ sobre $\phi(z)$ convergem rapidamente para $\psi_{\mathrm{j}}(z)$ a menos de uma constante. [34, 35]

$$
\lim _{\mathrm{n} \rightarrow \infty}\left(\frac{1}{\mathbf{H}-E_{\mathrm{j}}^{\prime}}\right)^{\mathrm{n}} \phi(z)=\lim _{\mathrm{n} \rightarrow \infty} \sum_{\mathrm{n}} a_{\mathrm{n}}\left(\frac{1}{E_{\mathrm{n}}-E_{\mathrm{j}}^{\prime}}\right)^{\mathrm{n}} \psi_{\mathrm{n}}(z)=A \psi_{\mathrm{j}}(z)
$$

Na verdade, para este método, não é necessário n muito grande, $\mathrm{n}$ da ordem de dezenas já leva autovetores e consequentemente autovalores tão ou mais precisos do que milhares de aplicações dos operadores de evolução em tempo imaginário.

A matriz $\left(\frac{1}{\mathbf{H}-E_{\mathrm{j}}^{\prime}}\right)$ é calculada com base na Eq. (4.31). Para tanto, notamos que

$$
\begin{aligned}
\mathbf{H}^{\mathrm{k}} \Psi_{\mathrm{j}}^{\mathrm{k}}= & -\frac{\hbar^{2}}{2 \Delta z^{2}}\left[\frac{\Psi_{\mathrm{j}+1}^{\mathrm{k}}-\Psi_{\mathrm{j}}^{\mathrm{k}}}{m_{\mathrm{j}+1 / 2}}-\frac{\Psi_{\mathrm{j}}^{\mathrm{k}}-\Psi_{\mathrm{j}-1}^{\mathrm{k}}}{m_{\mathrm{j}-1 / 2}}\right]+V_{\mathrm{j}}^{\mathrm{k}} \Psi_{\mathrm{j}}^{\mathrm{k}} \\
= & -\frac{\hbar^{2}}{2 \Delta z^{2}}\left[\frac{\Psi_{\mathrm{j}+1}^{\mathrm{k}}}{m_{\mathrm{j}+1 / 2}}-\frac{\Psi_{\mathrm{j}}^{\mathrm{k}}}{m_{\mathrm{j}+1 / 2}}-\frac{\Psi_{\mathrm{j}}^{\mathrm{k}}}{m_{\mathrm{j}-1 / 2}}+\frac{\Psi_{\mathrm{j}-1}^{\mathrm{k}}}{m_{\mathrm{j}-1 / 2}}\right]+V_{\mathrm{j}}^{\mathrm{k}} \Psi_{\mathrm{j}}^{\mathrm{k}} \\
= & -\frac{\hbar^{2}}{2 \Delta z^{2} m_{\mathrm{j}+1 / 2}} \Psi_{\mathrm{j}+1}^{\mathrm{k}}+\left[V_{\mathrm{j}}^{\mathrm{k}}+\frac{\hbar^{2}}{2 \Delta z^{2}}\left(\frac{1}{m_{\mathrm{j}+1 / 2}}+\frac{1}{m_{\mathrm{j}-1 / 2}}\right)\right] \Psi_{\mathrm{j}}^{\mathrm{k}} \\
& -\frac{\hbar^{2}}{2 \Delta z^{2} m_{\mathrm{j}-1 / 2}} \Psi_{\mathrm{j}-1}^{\mathrm{k}},
\end{aligned}
$$

ou seja, $\mathbf{H}^{\mathrm{k}}$ é uma matriz tridiagonal como em (4.24) ou (4.25), bastando subtrair dela a matriz $\mathbb{1} E_{\mathrm{j}}^{\prime}$ e inverter o resultado.

A convergência do método será são boa quanto melhor for a aproximação inicial para $\epsilon$ ou $E_{\mathrm{j}}^{\prime}$. Na prática, avaliamos o potencial em uma região onde é possível que existam estados ligados, chamamos o mínimo dessa região de $\epsilon_{0}$ e o máximo de $\epsilon_{\mathrm{m}}$. Criamos uma sequência com m termos

$$
\epsilon_{\mathrm{i}}=\mathrm{i} \frac{\left(\epsilon_{\mathrm{m}}-\epsilon_{0}\right)}{\mathrm{m}}, \mathrm{i}=0, \ldots, \mathrm{m}-1,
$$

que utilizamos sucessivamente no procedimento descrito pela Eq. (4.65). Em geral, valores diferentes de $\epsilon_{\mathrm{i}}$ podem levar ao mesmo $\psi_{\mathrm{j}}(z)$, sendo então necessário remover os repetidos. Também é possível que nenhum dos valores $\epsilon_{\mathrm{i}}$ convirja para um determinado $\psi_{\mathrm{k}}(z)$. Quanto maior m, maior a chance de todos os estados ligados serem encontrados.

Tanto a construção de $\phi(z)$ quanto do próprio operador hamiltoniano $\mathbf{H}$ dependem de discretizações do espaço e tempo como sugerimos nas Eqs. (4.1) e (4.2), respectivamente. Para o caso de heteroestruturas semicondutoras com potenciais do tipo poço quântico, ou semelhantes, muitas vezes é interessante destinar mais pontos para a região de interesse (poço) e menos para as extremidades. Em geral isso resulta em uma melhor representação da região ativa, com menor custo computacional. Entretanto, observamos que sempre que existe em cena algo que deixe as extremidades com algum desnível de energia, situação muito comum quando existe a aplicação de um campo elétrico estático, a convergência deste método se torna muito melhor para malhas igualmente espaçadas. 


\section{Validação dos Métodos Numéricos Através de Problemas com Solução Conhecida}

Neste capítulo, vamos utilizar os métodos descritos no capítulo anterior para calcular a evolução temporal, os autoestados e autovalores de alguns sistemas quânticos que têm solução analítica ou que já foram abordados numericamente por autores da área.[1, 6] A intenção aqui é demonstrar que os métodos desenvolvidos neste trabalho são válidos para qualquer tipo de potencial e que reproduzem de forma precisa os resultados analíticos. Com isso podemos sugerir que a qualidade das soluções para potenciais sem solução analítica conhecida será a mesma.

Todos os cálculos são realizados utilizando unidades atômicas, onde a massa do elétron $\left(m_{\mathrm{e}}\right)$, a carga elementar $(q)$, a constante de Planck reduzida $(\hbar)$ e a constante de Coulomb $\left(k_{\mathrm{e}}\right)$ são todas iguais a unidade. Nesse caso, a equação de Schrödinger (2.14) fica com a seguinte forma:

$$
-\frac{1}{2} \partial_{z}^{2} \Psi\left(z_{\mathrm{au}}, t_{\mathrm{au}}\right)+V_{\mathrm{au}}\left(z_{\mathrm{au}}\right) \Psi\left(z_{\mathrm{au}}, t_{\mathrm{au}}\right)=i \partial_{t} \Psi\left(z_{\mathrm{au}}, t_{\mathrm{au}}\right),
$$

onde as coordenadas espaciais, temporais e a energia do potencial precisam ser convertidas para o sistema de unidades atômicas, conforme Tabela 2.

Tabela 2: Relação entre grandezas no sistema de unidades atômicas (UA) e no sistema internacional (SI) de unidades.

\begin{tabular}{lccc}
\hline Unidade & Símbolo & UA & SI \\
\hline Comprimento (Raio de Bohr) & $a_{0}$ & 1,0 & $5,2917721092(17) \times 10^{-11} \mathrm{~m}$ \\
Energia (de Hartree) & $E_{\mathrm{H}}$ & 1,0 & $4,35974417(75) \times 10^{-18} \mathrm{~J}$ \\
Tempo & $\hbar / E_{\mathrm{H}}$ & 1,0 & $2,418884326505(16) \times 10^{-17} \mathrm{~s}$ \\
Massa (do elétron livre) & $m_{\mathrm{e}}$ & 1,0 & $9,10938291(40) \times 10^{-31} \mathrm{~kg}$ \\
\hline
\end{tabular}

\subsection{Evolução Temporal: Pacote de Onda Plana}

Nesta seção testamos os três métodos de evolução temporal (Runge-Kutta, CrankNicolson e Pseudo-Espectral) para evoluir um pacote de onda gaussiano. Como o pacote de onda é representado de forma discreta no espaço com uma precisão $\Delta z$ e cada passo de evolução avança $\Delta t$ no tempo, variamos estes dois parâmetros, também levando em conta os efeitos de borda, com o objetivo de identificar os melhores valores a serem utilizados para cada método e para cada tamanho de sistema. Ao final, fizemos uma análise que nos permite assumir que o método Pseudo-Espectral é muito mais rápido e preciso do que os outros dois em praticamente todas as situações. 
Um pacote de onda plana é uma construção teórica onde uma partícula pode ser representada por uma função de onda composta por uma combinação de autoestados de uma hamiltoniana com $V(z)=0$. Os autoestados dessa hamiltoniana são ondas planas que se propagam da direita para a esquerda ou vice-versa. O pacote de onda seria então uma combinação muito cuidadosa de autoestados que se cancelam ou se somam em diferentes posições do espaço dando origem à esta partícula relativamente bem localizada.

Vamos adotar um pacote de onda gaussiano, que representamos por [36]

$$
\Psi(z, 0)=\frac{1}{\sqrt[4]{2 \pi \sigma_{z}^{2}}} \exp \left(i k_{0} z-\frac{\left(z-z_{0}\right)^{2}}{4 \sigma_{z}^{2}}\right)
$$

onde $k_{0}$ é o vetor de onda da partícula com momento $p_{0}=\hbar k_{0}$ e energia inicial $E_{0}$ dada pela relação: $E_{0}\left(k_{0}\right)=\hbar \omega\left(k_{0}\right)=\frac{\hbar^{2} k_{0}^{2}}{2 m}$.

Existe uma solução analítica para a evolução temporal desse pacote de onda que é dada por [40]

$$
\Psi(z, t)=\int_{-\infty}^{+\infty} \mathcal{F}[\Psi(z, 0)] e^{i \varphi(k, t)} d k
$$

onde $\mathcal{F}[\Psi(z, 0)]$ é a transformada de Fourier de $\Psi(z, 0)$ e $\varphi(k, t)=k z-\omega(k) t$.

Nós definimos um pacote de onda arbitrário, evoluindo-o no tempo de acordo com as propriedades listadas na Tabela 3, e aplicamos as técnicas numéricas descritas no Capítulo 4. A solução analítica fornecida pela Eq. (5.3) foi utilizada para avaliar a qualidade de cada evolução.

Tabela 3: Características do pacote de onda analisado.

\begin{tabular}{ccc}
\hline$\left\langle z_{\mathrm{i}}\right\rangle$ & $\left\langle z_{\mathrm{f}}\right\rangle$ & $E_{0}$ \\
\hline$-20,0 \AA$ & $+20,0 \AA$ & $150,0 \mathrm{eV}$ \\
\hline
\end{tabular}

Para cada um dos métodos (Crank-Nicolson, Runge-Kutta e Pseudo-Espectral) foram variados:

- (linearmente) o tamanho $L$ da malha espacial entre 100 e $1000 \AA$, para identificar a influência de efeitos de borda;

- (exponencialmente) o número de pontos $N$ do espaço entre 256 e 2048, para identificar a influência do nível de amostragem espacial;

- (exponencialmente) o passo de tempo $\Delta t$ entre $1 \times 10^{-20}$ e $1 \times 10^{-16}$ segundos, para identificar a estabilidade do método.

A qualidade ${ }^{1}$ de cada evolução foi analisada de acordo com os seguintes parâmetros:

1 Estamos usando a notação $\mathbf{Q}$ para a métrica que indica qualidade e $\mathbf{P}$ para a métrica de performance. 
- $\mathbf{Q}_{1}$ a conservação da norma [Eq. (5.5)], ou seja, a integral de $|\Psi(z, t)|^{2}$ em todo o espaço não pode variar;

- $\mathbf{Q}_{\mathbf{2}}$ o desvio padrão [Eq. (5.7)] do pacote de onda deve aumentar, mas de acordo com o aumento observado na solução analítica;

- Q3 não deve surgir obliquidade [Eq. (5.8)] durante a evolução;

- $\mathbf{P}_{\mathbf{1}}$ o tempo de processamento, sendo este um parâmetro mais relacionado à performance do que à qualidade em si.

Computacionalmente falando, tanto as evoluções numéricas quanto as evoluções analíticas são (ou pelo menos deveriam ser) transformações unitárias $U: \mathbb{C}^{N} \rightarrow \mathbb{C}^{N}$, ou seja, recebem um vetor $\Psi(z, 0)$ do espaço $\mathbb{C}^{N}$ e devolvem um vetor $\Psi(z, t)$ no mesmo espaço. Sendo assim, analisar a qualidade de um pacote de onda evoluído numericamente implica observar sua semelhança ponto a ponto com um pacote de onda evoluído analiticamente e também garantir que as propriedades relacionadas à distribuição de probabilidade que ela representa sejam mantidas. Por esta razão adotamos descrições estatísticas para fazer as avaliações de qualidade.

De acordo com a terminologia utilizada em Estatística, a expressão que fornece o $n$-ésimo momento da variável $z$ submetido à função densidade de probabilidade $|\Psi(z, t)|^{2}$ é dada por: [41]

$$
\left\langle z^{n}\right\rangle(t)=\int_{-\infty}^{+\infty} \Psi^{\dagger}(z, t) z^{n} \Psi(z, t) d z
$$

Tomamos liberdade de identificar o momento "zero", como a norma $A(t)$ da função densidade de probabilidade, isto é:

$$
A(t)=\left\langle z^{0}\right\rangle(t)
$$

Em Estatística, esse valor deve, em geral, ser igual à unidade, mas em Mecânica Quântica isso só é verdade se a distribuição corresponder à um autoestado estacionário de uma base de estados ortonormais. O pacote de onda com o qual lidamos é uma sobreposição de estados estacionários, então é natural que sua norma seja diferente da unidade.

O primeiro momento em torno da média, Eq. (5.6), representa a posição média $\mu(t)$, ou mais provável, de se encontrar o pacote de onda, ou seja,

$$
\mu(t)=\frac{\langle z\rangle(t)}{A(t)}
$$

O desvio padrão $\sigma(t)$ é definido como sendo a dispersão do segundo momento em torno da média, isto é: [41]

$$
\sigma(t)=\frac{\sqrt{\left\langle z^{2}\right\rangle(t)-\mu^{2}(t)}}{A(t)} .
$$


A obliquidade $\gamma(t)$, que é uma medida da simetria da função densidade de probabilidade, é definida como: [41]

$$
\gamma(t)=\frac{\left\langle z^{3}\right\rangle(t)-3 \mu(t) \sigma^{2}(t)-\mu^{3}(t)}{\sigma^{3}(t)} .
$$

Na Figura 7 ilustramos como o desvio padrão e a obliquidade alteram a forma de uma função densidade de probabilidade.

Para que possamos combinar os parâmetros de qualidade em uma única métrica, utilizamos a distância de Minkowski [42], definida na Eq. (5.13), que representa o quão distante dois vetores estão entre si. Seria possível utilizar somente esta métrica para comparar os pacotes de onda evoluídos numericamente e analiticamente, mas perderíamos a sensibilidade em relação aos parâmetros de qualidade que queremos observar.

A ideia aqui é definir um vetor:

$$
X(t)=(\mathcal{A}(t), \mathcal{S}(t), \mathcal{G}(t))
$$

que pode ser calculado em qualquer instante de tempo para um pacote de onda evoluído
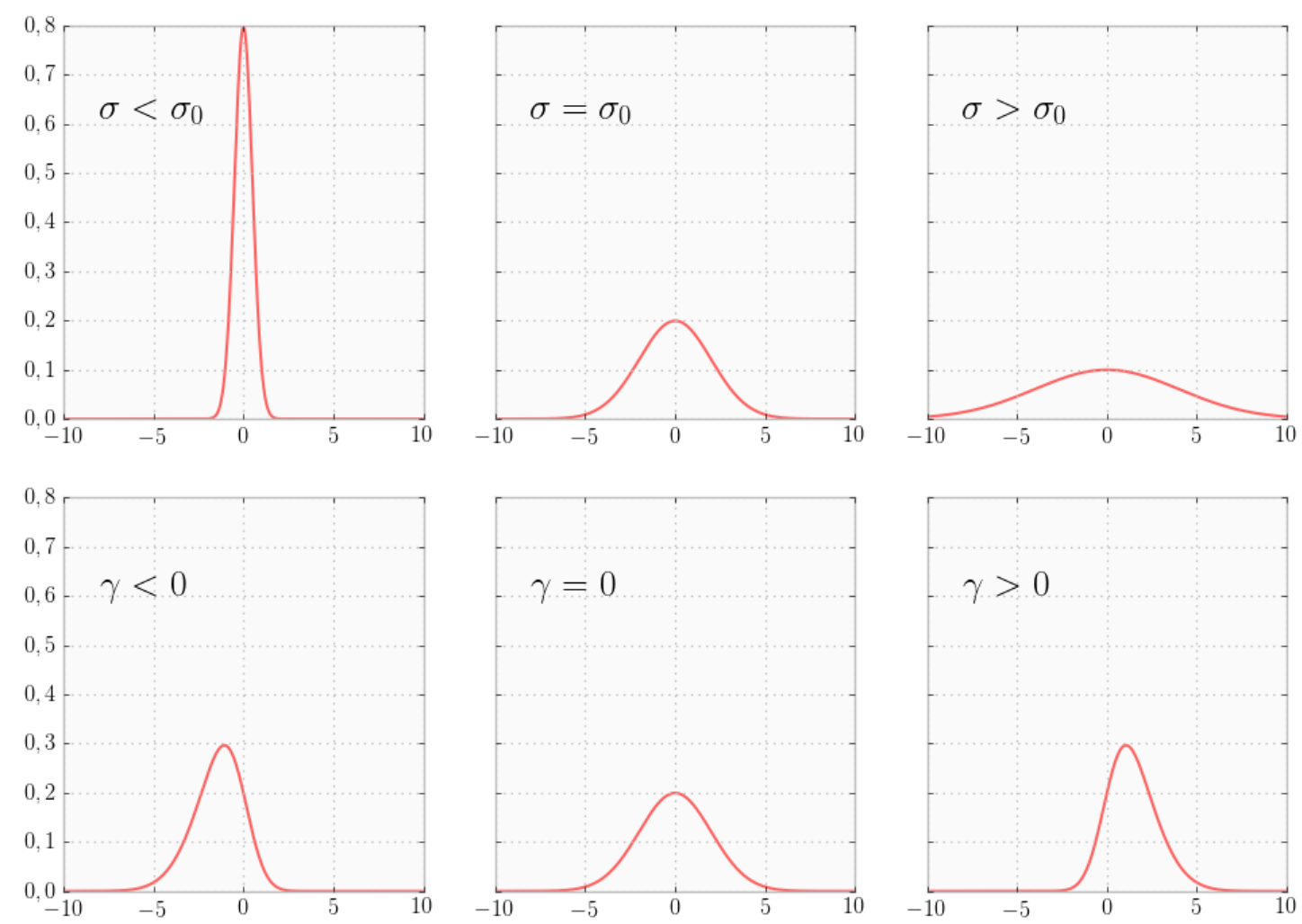

Figura 7: Nos três quadros superiores é mostrado o efeito do desvio padrão em uma distribuição de probabilidade. Quanto menor o desvio padrão, mais estreita, e quando maior o desvio padrão, mais larga é a distribuição de probabilidade. Nos três quadros inferiores é mostrado o efeito da obliquidade em uma distribuição de probabilidade. Uma obliquidade menor do que zero implica em uma distribuição com maior área do lado esquerdo, ao passo que uma obliquidade maior do que zero implica em uma distribuição com maior área do lado direito. 
numericamente ou analiticamente, onde:

$$
\begin{aligned}
& \mathcal{A}(t)=\frac{A(t)}{A(0)}, \\
& \mathcal{S}(t)=\sigma(t), \\
& \mathcal{G}(t)=\gamma(t) .
\end{aligned}
$$

Se chamarmos de $X(t)$ o vetor referente ao pacote de onda evoluído numericamente e de $Y(t)$ o vetor referente ao pacote de onda evoluído analiticamente, então a distância de Minkowski com parâmetro $p$ é dada pela expressão:

$$
D_{p}(X(t), Y(t))=\left(\sum_{i=1}^{3}\left|X_{i}(t)-Y_{i}(t)\right|^{p}\right)^{1 / p} .
$$

É importante notar que os parâmetros $\mathcal{A}(t), \mathcal{S}(t)$ e $\mathcal{G}(t)$ possuem dimensões e escalas diferentes, fazendo-se necessário um ajuste de escala que pode ser feito através de:

$$
\begin{aligned}
& \mathbb{A}(t)=\frac{\mathcal{A}(t)-\langle\mathcal{A}(t)\rangle}{\sigma(\mathcal{A}(t))}, \\
& \mathbb{S}(t)=\frac{\mathcal{S}(t)-\langle\mathcal{S}(t)\rangle}{\sigma(\mathcal{S}(t))}, \\
& \mathbb{G}(t)=\frac{\mathcal{G}(t)-\langle\mathcal{G}(t)\rangle}{\sigma(\mathcal{G}(t))},
\end{aligned}
$$

Veja que cada um destes valores são calculados para cada resultado final (numérico ou analítico), então estas médias e desvios-padrão referem-se ao conjunto de todos os resultados que serão obtidos, ou seja, são calculados sobre o universo das 350 combinações de $\{L, N, \Delta t\}$.

Sendo assim, a distância de Minkowski (que em geral iremos calcular usando $p=3$ ) assume a forma final:

$$
D_{3}(\mathbb{X}(t), \mathbb{Y}(t))=\left(\sum_{i=1}^{3}\left|\mathbb{Z}_{i}(t)-\mathbb{Y}_{i}(t)\right|^{3}\right)^{1 / 3}
$$

A vantagem de se utilizar a métrica de Minkowski é que ela valoriza qualquer uma das medidas que se tornar grande em relação às demais. Isso é interessante porque de nada adianta uma combinação de parâmetros $\{L, N, \Delta t\}$ que conserve a norma e aumente o desvio padrão de forma adequada, mas que torne a onda completamente oblíqua, o mesmo valendo para um aumento incorreto no desvio padrão ou não conservação da norma. A escolha do parâmetro $p=3$ é totalmente arbitrária, não há nada de especial nesse caso. 
Prosseguindo com nossa análise, variando os parâmetros $\{L, N, \Delta t\}$, obtivemos 350 combinações de resultados para o método Pseudo-Espectral. Entretanto para os métodos de Runge-Kutta e Crank Nicolson, algumas combinações de parâmetros foram evitadas para não violar a estabilidade dos métodos. Além disso, para estes dois últimos métodos, ocorreram muitas vezes estouro de pilha e divergência do algoritmo, mesmo para parâmetros que deveriam ser estáveis.

Na Figura 8 mostramos a métrica de Minkowski $D_{3}(\mathcal{X}, Y)$ em função do tempo de processamento, para cada um dos métodos analisados. Este gráfico mostra que não existe uma relação simples entre tempo de processamento e a qualidade dos algoritmos, ou seja, existem parâmetros que convergem com ótima qualidade em um tempo curto e parâmetros que levam a uma baixa qualidade levando um tempo longo. Isso é bom, pois indica a possibilidade de encontrarmos bons parâmetros que levem rapidamente à um resultado de qualidade.

Apesar de os gráficos relacionados aos métodos de Runge-Kutta e Crank-Nicolson serem bastante parecidos, o gráfico relacionado ao método Pseudo-Espectral revela que
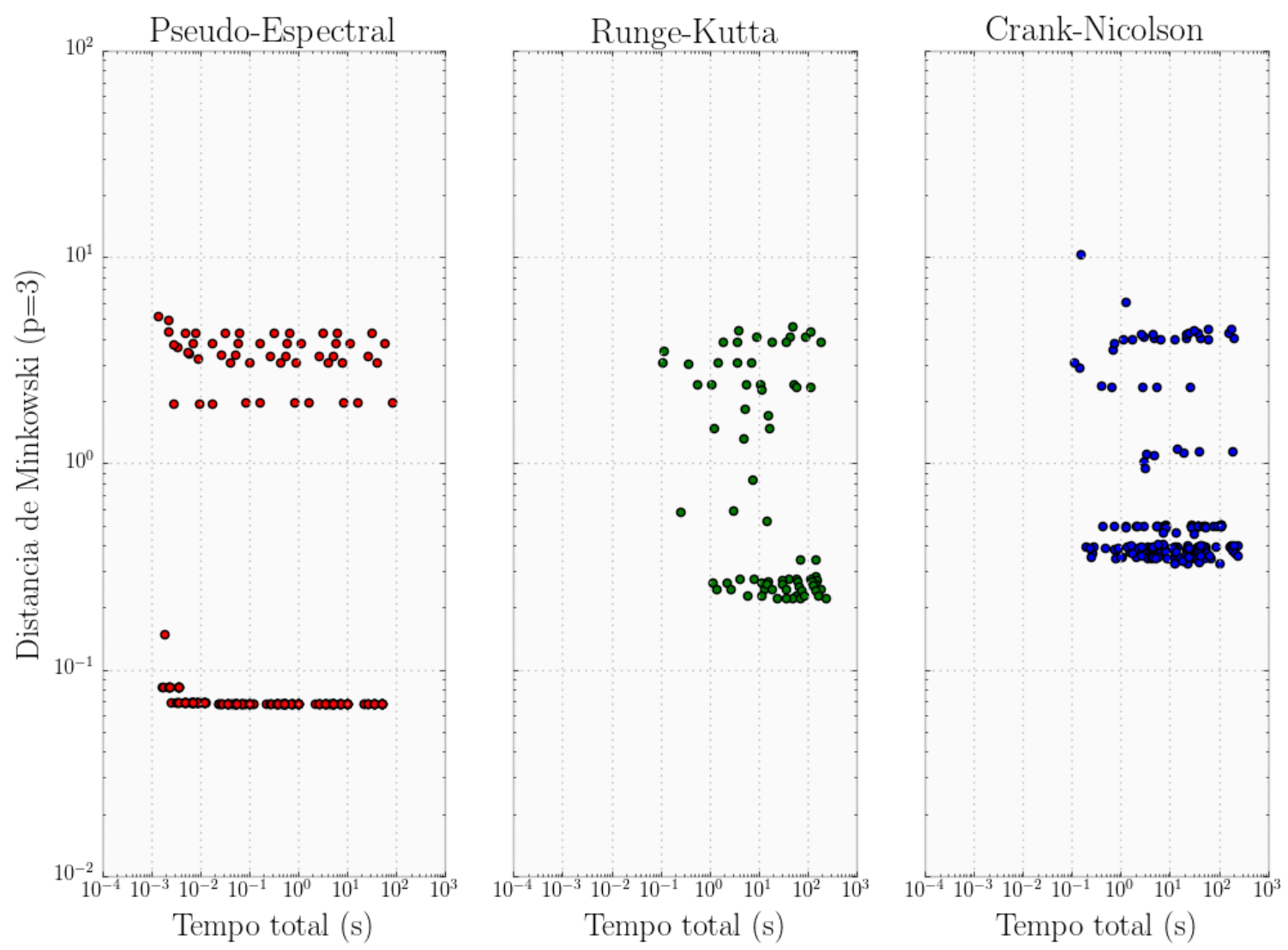

Figura 8: Distância de Minkowski dada pela Equação (5.17) para os três métodos utilizados (PseudoEspectral, Runge-Kutta e Crank-Nicolson). Da maneira como estruturamos, quanto menor a distância de Minkowski, melhor a qualidade do método. Cada ponto representa uma combinação de parâmetros $\{L, N, \Delta t\}$, que é representada somente pelo tempo que o algoritmo leva para convergir e a qualidade do resultado. 
este método permite a obtenção de resultados de maior qualidade em um curto período de tempo se comparado com os demais. Isto pode ser visto observando o canto inferior esquerdo dos gráficos na Figura 8.

O algoritmo utilizado para gerar a Figura 8 pode ser encontrado no Anexo A.1.

\subsection{Cálculo de Níveis de Energia: Oscilador Harmônico Quântico}

Embora na seção anterior tenhamos verificado a melhor precisão e performance do método Pseudo-Espectral na evolução temporal de um sistema quântico, ainda precisamos conferir sua qualidade em relação a obtenção de autovalores e autovetores, o que se dá através da evolução em tempo imaginário (para obter o estado fundamental) juntamente com a ortogonalização de Gram-Schmidt (para obter os estados excitados).

Nesta seção testamos o método Pseudo-Espectral para obtenção das autofunções e autoenergias de um problema com solução analítica conhecida. É feito um estudo do tempo de convergência do algoritmo a fim de atingir máxima precisão em relação aos resultados analíticos.

O oscilador harmônico quântico é amplamente estudado nos livros texto de Mecânica Quântica não só por ser um caso excepcional para o qual existe uma solução analítica, mas por ser um boa aproximação para muitas situações em que, na vizinhança de um ponto, um potencial é aproximadamente parabólico [40].

Em uma dimensão, o potencial correspondente ao oscilador harmônico quântico, é descrito na equação:

$$
V(z)=\frac{m_{\mathrm{e}} \omega^{2} z^{2}}{2}
$$

onde $m_{\mathrm{e}}=$ autoenergias deste potencial são obtidas através de

$$
E_{\mathrm{n}}=\hbar \omega\left(\mathrm{n}+\frac{1}{2}\right)
$$

e os autoestados através de

$$
\psi_{\mathrm{n}}(z)=\frac{1}{\sqrt{2^{\mathrm{n}} \mathrm{n} !}}\left(\frac{m_{\mathrm{e}} \omega}{\pi \hbar}\right)^{1 / 4} \exp \left(-\frac{m_{\mathrm{e}} \omega z^{2}}{2 \hbar}\right) H_{\mathrm{n}}\left(\sqrt{\left.\frac{m_{\mathrm{e}} \omega}{\hbar} z\right)},\right.
$$

como é possível encontrar em praticamente todo texto sobre Mecânica Quântica [40]. As funções $H_{\mathrm{n}}(z)$ são polinômios de Hermite,

$$
H_{\mathrm{n}}(z)=(-1)^{\mathrm{n}} e^{z^{2}} \frac{d^{\mathrm{n}}}{d z^{\mathrm{n}}} e^{-z^{2}}
$$

Utilizamos o método de evolução Pseudo-Espectral em tempo imaginário para obter as autoenergias e autoestados do potencial na Eq. (5.18). Não há ganho de precisão 
ao utilizar os métodos de Crank-Nicolson ou Range-Rutta, há somente um grande esforço computacional que se traduz em um longo tempo de processamento.

Definimos uma malha espacial de comprimento $L=100 \AA$, dividida em $N=2048$ pontos. A frequência $\omega$ utilizada corresponde à um comprimento de onda $\lambda=8,1 \mu \mathrm{m}$, através de $\omega=2 \pi c / \lambda$.

Como sabemos que esta combinação de $\{L, N, \Delta t\}$ é estável para o método PseudoEspectral, comparamos os autoestados obtidos numericamente $\left(\left\{\varphi_{n}(z)\right\}\right)$ e o autoestados analíticos $\left(\left\{\psi_{n}(z)\right\}\right)$ dados pela Eq. (5.20) utilizando somente a distância euclidiana quadrática

$$
D_{\mathrm{e}}\left(\varphi_{\mathrm{n}}, \psi_{\mathrm{n}}\right)=\sqrt{\sum_{\mathrm{i}}\left|\varphi_{\mathrm{n}}\left(z_{\mathrm{i}}\right)-\psi_{\mathrm{n}}\left(z_{\mathrm{i}}\right)\right|^{2}}
$$

onde o índice i corresponde a cada ponto da malha espacial discreta utilizada.

Na Figura 9 mostramos os primeiros seis autoestados e autovalores obtidos numericamente.

Nas Figuras 10 e 11 mostramos a diminuição do erro dado pela Eq. (5.22) em função do tempo de processamento e do número de iterações, respectivamente. Em ambas

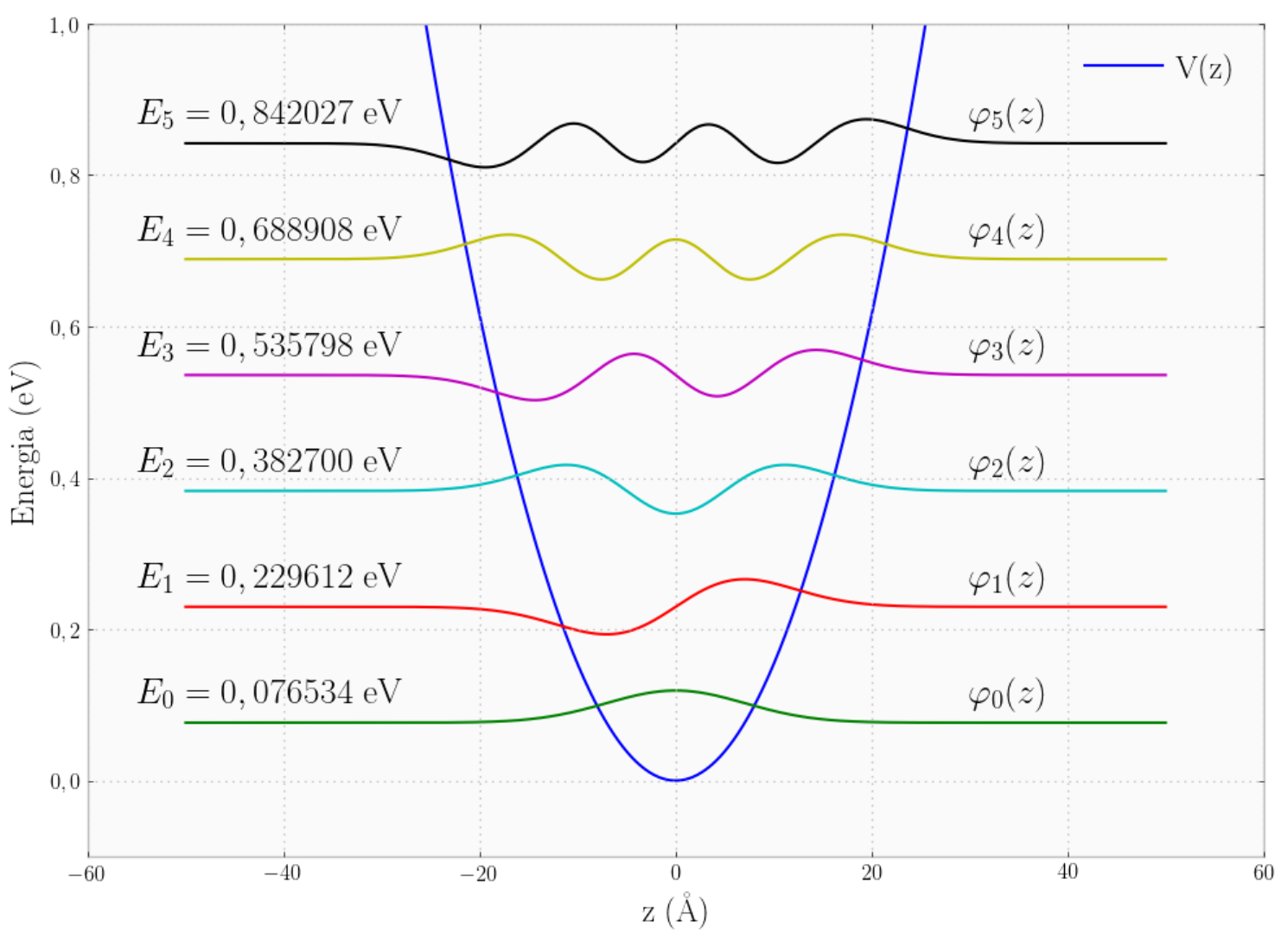

Figura 9: Autoestados e autovalores para um elétron de massa $m_{\mathrm{e}}$ em um potencial tipo oscilador harmônico obtidos através do método Pseudo-Espectral. Nestes cálculos utilizamos $\omega=2,3 \times 10^{14} \mathrm{rad} / \mathrm{s}$, equivalente a um comprimento de onda $\lambda=8,1 \mu \mathrm{m}$. 


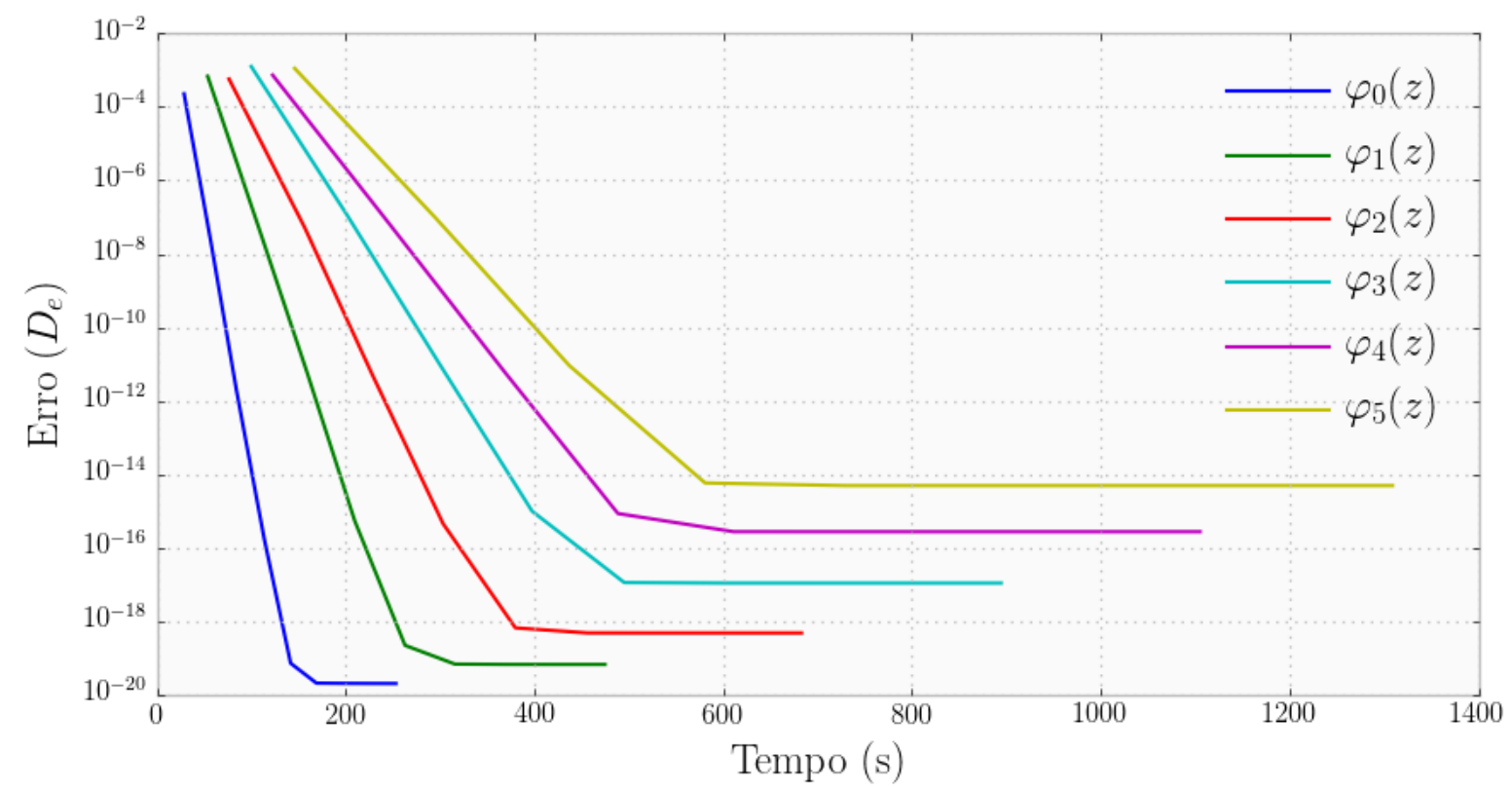

Figura 10: Erro cometido no cálculo dos autoestados para um elétron de massa $m_{\mathrm{e}}$ submetido ao potencial oscilador harmônico quântico dado pela Eq. (5.22) com $\omega=2,3 \times 10^{14} \mathrm{rad} / \mathrm{s}$ (equivalente a um comprimento de onda $\lambda=8,1 \mu \mathrm{m})$ em função do tempo em segundos para calcular cada autoestado.

as figuras é possível ver que o erro cometido no cálculo de cada autoestado $\varphi_{\mathrm{i}}(z)$ se propaga para todos os outros estados $\varphi_{\mathrm{j}}(z) \operatorname{com} \mathrm{j}>\mathrm{i}$. Isto se deve à utilização da ortogonalização de Gram-Schmidt, Eqs. (4.62), que precisa ortogonalizar cada autoestado $\varphi_{\mathrm{j}}(z)$ em relação a todas os outros autoestados $\varphi_{\mathrm{i}}(z), \operatorname{com} \mathrm{j}>\mathrm{i}$.

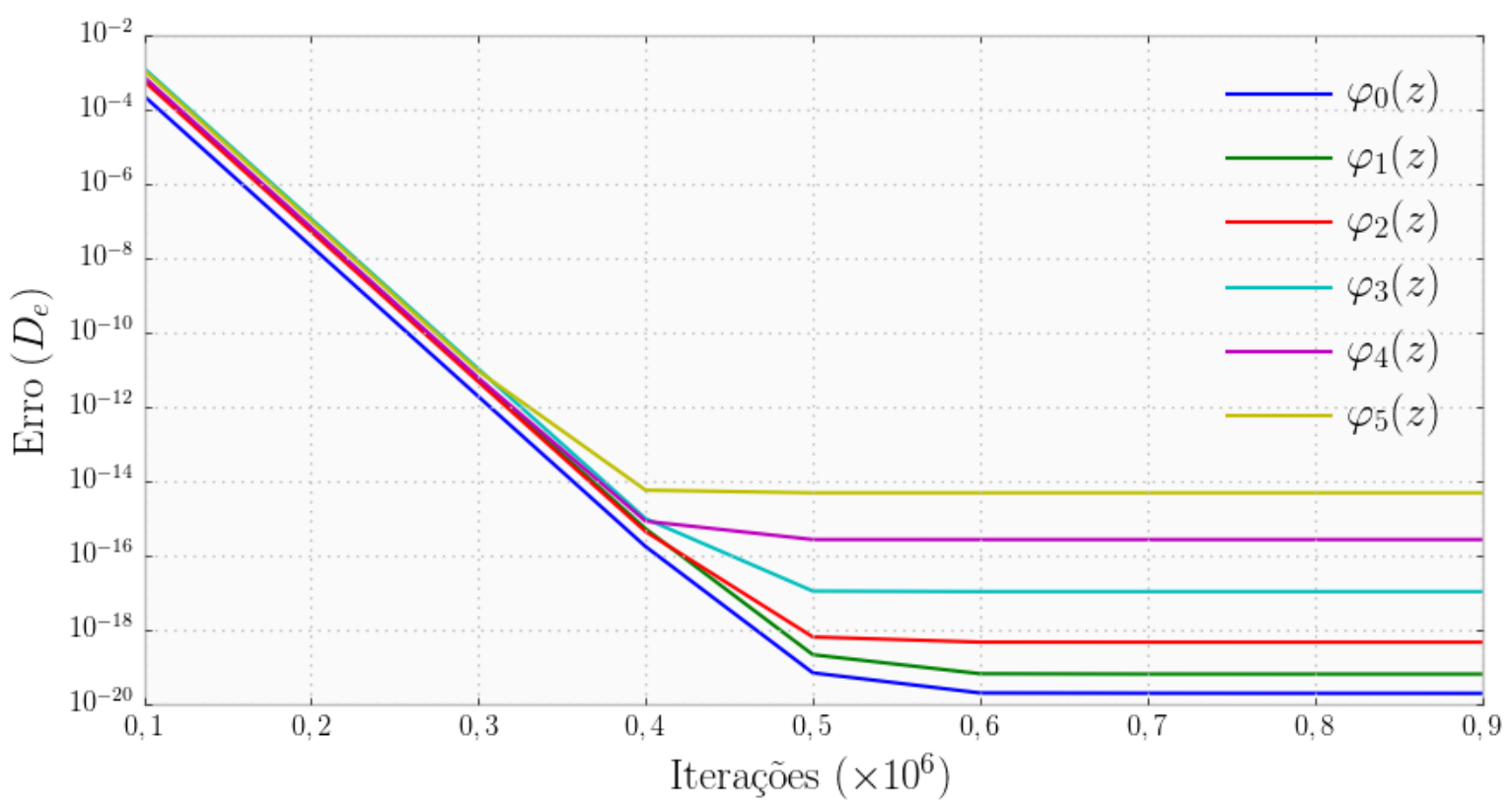

Figura 11: Erro cometido no cálculo dos autoestados para um elétron de massa $m_{\mathrm{e}}$ submetido ao potencial oscilador harmônico quântico dado pela Eq. (5.22) com $\omega=2,3 \times 10^{14} \mathrm{rad} / \mathrm{s}$ (equivalente a um comprimento de onda $\lambda=8,1 \mu \mathrm{m}$ ) em função do número de iterações para calcular cada autoestado. 
O algoritmo utilizado na obtenção desses autoestados e autovalores pode ser verificado no Anexo A.2.

A grande precisão obtida, sobretudo para os estados de menor energia, revela que o método Pseudo-Espectral é muito eficiente na obtenção de autovalores e autovetores, entretanto, o tempo de processamento neste caso é um pouco longo. Este foi um primeiro teste, onde confrontamos o método com a solução analítica de um potencial bastante específico. Vamos na próxima seção analisar um potencial mais parecido com aqueles observados em heteroestruturas semicondutoras do tipo poço quântico.

\subsection{Cálculo de Níveis de Energia: Poço Quântico}

Na seção anterior verificamos a grande precisão do método Pseudo-Espectral em relação a solução analítica do oscilador harmônico quântico. Entretanto, isto só significa que ele está de acordo com a Mecânica Quântica pura. Nesta dissertação estamos interessados em sistemas físicos onde precisaremos introduzir termos semi-clássicos e até mesmo fenomenológicos, por esta razão precisamos verificar se o método segue preciso e eficiente quando aplicado a sistemas um pouco mais complexos. Nesta seção analisamos um poço quântico simples, porque além de possuir solução analítica, é um potencial bastante semelhante aos potenciais observados em heteroestruturas semicondutoras.

Um poço quântico é uma construção teórica a respeito de uma região no espaço onde o potencial é representado por

$$
V(z)= \begin{cases}0, & |z| \leq a / 2 \\ V_{\mathrm{b}}, & |z|>a / 2\end{cases}
$$

onde $a$ é a largura do poço. Esta estrutura demonstrou ser uma ótima descrição para algumas heteroestruturas semicondutoras.[11] Dos textos de Mecânica Quântica sabemos que existem duas equações transcendentais que os autovalores $E_{\mathrm{n}}$ deste potencial precisam obedecer: [7]

$$
\begin{aligned}
& \tan \left(\sqrt{\frac{a^{2} m_{\mathrm{e}}^{*} E_{\mathrm{n}}}{2 \hbar^{2}}}\right)=\sqrt{\frac{\left(V_{\mathrm{b}}-E_{\mathrm{n}}\right)}{E_{\mathrm{n}}}}, \\
& \cot \left(\sqrt{\frac{a^{2} m_{\mathrm{e}}^{*} E_{\mathrm{n}}}{2 \hbar^{2}}}\right)=-\sqrt{\frac{\left(V_{\mathrm{b}}-E_{\mathrm{n}}\right)}{E_{\mathrm{n}}}} .
\end{aligned}
$$

Nós podemos resolver as equações (5.24a) e (5.24b) utilizando o método de NewtonRaphson para encontrar raízes de equações se fizermos:

$$
f\left(E_{\mathrm{n}}\right)=\tan \left(\sqrt{\frac{a^{2} m_{\mathrm{e}}^{*} E_{\mathrm{n}}}{2 \hbar^{2}}}\right)-\sqrt{\frac{\left(V_{\mathrm{b}}-E_{\mathrm{n}}\right)}{E_{\mathrm{n}}}}=0,
$$




$$
g\left(E_{\mathrm{n}}\right)=\cot \left(\sqrt{\frac{a^{2} m_{\mathrm{e}}^{*} E_{\mathrm{n}}}{2 \hbar^{2}}}\right)+\sqrt{\frac{\left(V_{\mathrm{b}}-E_{\mathrm{n}}\right)}{E_{\mathrm{n}}}}=0 .
$$

Nós escolhemos analisar um potencial com $V_{\mathrm{b}}=1,0 \mathrm{eV}$ e variamos a largura $a$ entre $10.0 \AA$ e $80,0 \AA$. Na Figura 12 mostramos as autoenergias obtidas através das raízes das equações (5.25a) e (5.25b), alguns pontos posicionados aleatoriamente fora das curvas principais se devem a divergência do algoritmo de Newton-Raphson utilizado. Deixamos propositalmente para evidenciar o tipo de erro em que se pode incorrer mesmo através de uma abordagem pseudo-analítica, ou seja, onde a solução para um problema com solução analítica fica dependente de um último passo, no caso a solução de raízes, através de uma técnica numérica bastante confiável.

Na Figura 13 mostramos as autoenergias obtidas através do método PseudoEspectral. É interessante obsevar que não ocorrem pontos posicionados aleatoriamente fora das curvas. Na Figura 14 mostramos as autoenergias da Figura 12 sobrepostas com as da Figura 13, para fins de comparação visual.

Em nosso algoritmo utilizamos a ortogonalização de Gram-Schmidt para obter estados além do fundamental fornecido pelo método Pseudo-Espectral simples. Em princípio, esta ortogonalização nos permite calcular autoestados ortogonais indefinidamente. Acima do topo do poço existe um contínuo de estados, de modo que se prosseguirmos utilizando nossa malha espacial que é finita de largura $L$, incorreríamos em um erro

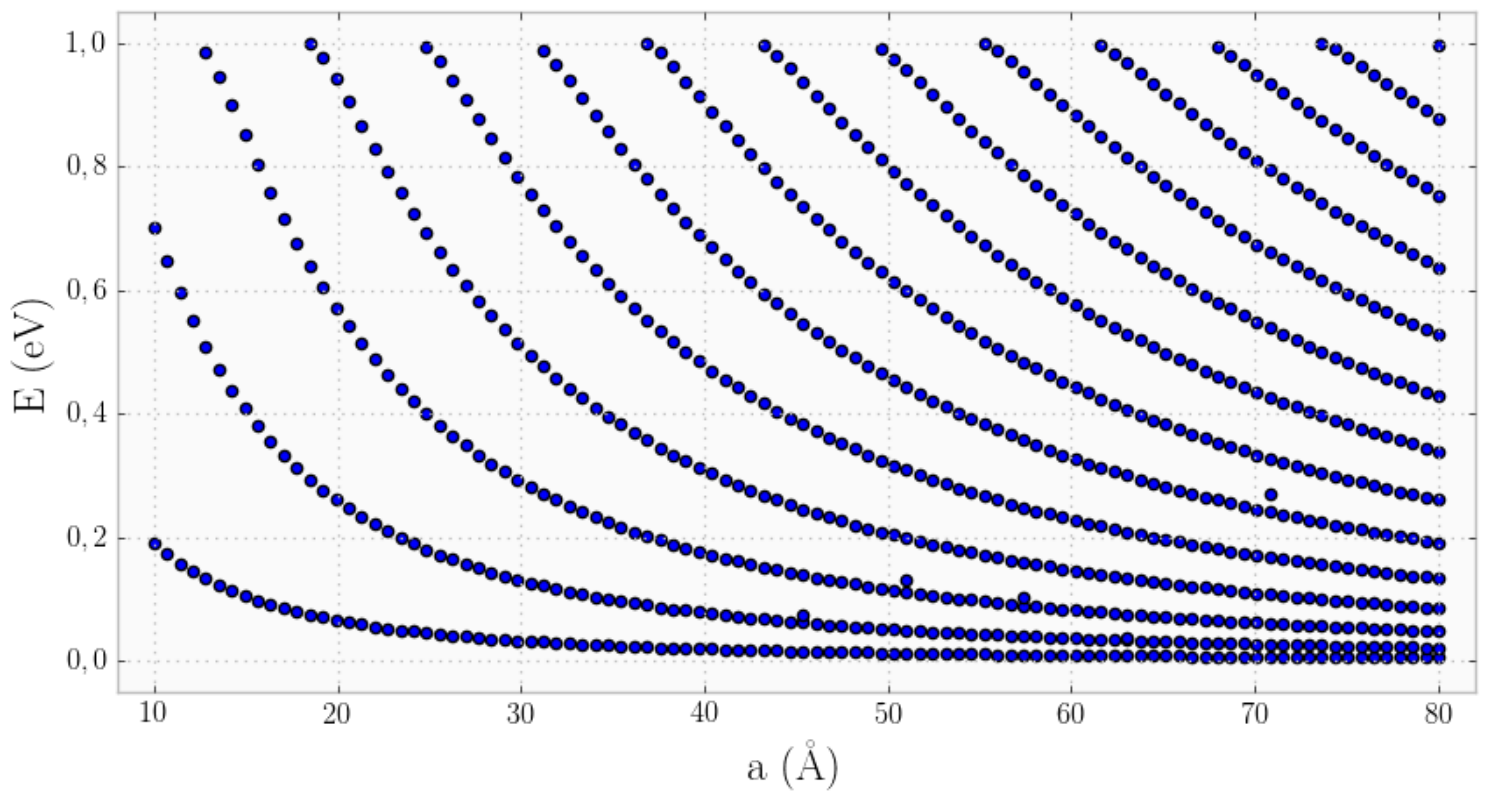

Figura 12: Autoenergias de estados possíveis para um elétron de massa $m_{\mathrm{e}}$ dentro de um poço quântico de altura $V_{\mathrm{b}}=1,0 \mathrm{eV}$ em função da largura, obtidos através da solução das raízes das equações (5.25a) e (5.25b). Alguns pontos posicionados aleatoriamente entre as curvas se devem a divergência do algoritmo de Newton-Raphson utilizado. 


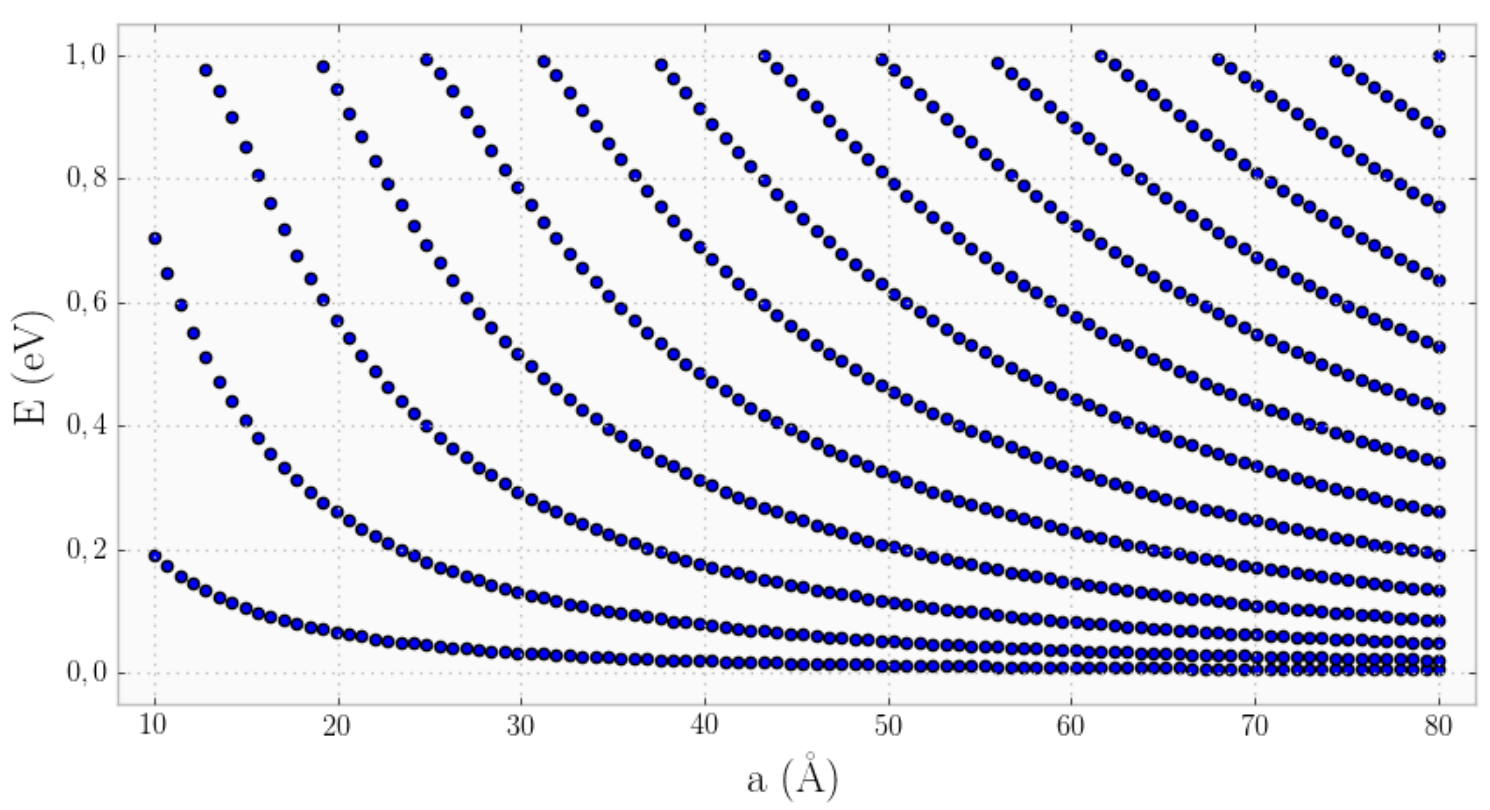

Figura 13: Autoenergias de estados possíveis dentro de um poço quântico de altura $V_{\mathrm{b}}=1,0$ eV em função da largura, obtidos através do método Pseudo-Espectral.

devido ao fato de o método Pseudo-Espectral supor condições de contorno periódicas. Portanto, é importante parar de calcular novos estados assim que a autoenergia de um novo nível obtido ultrapassar o topo do poço. Por simplicidade e rapidez, nosso algoritmo primeiro calcula o número de estados através da solução analítica, para ter uma estimativa

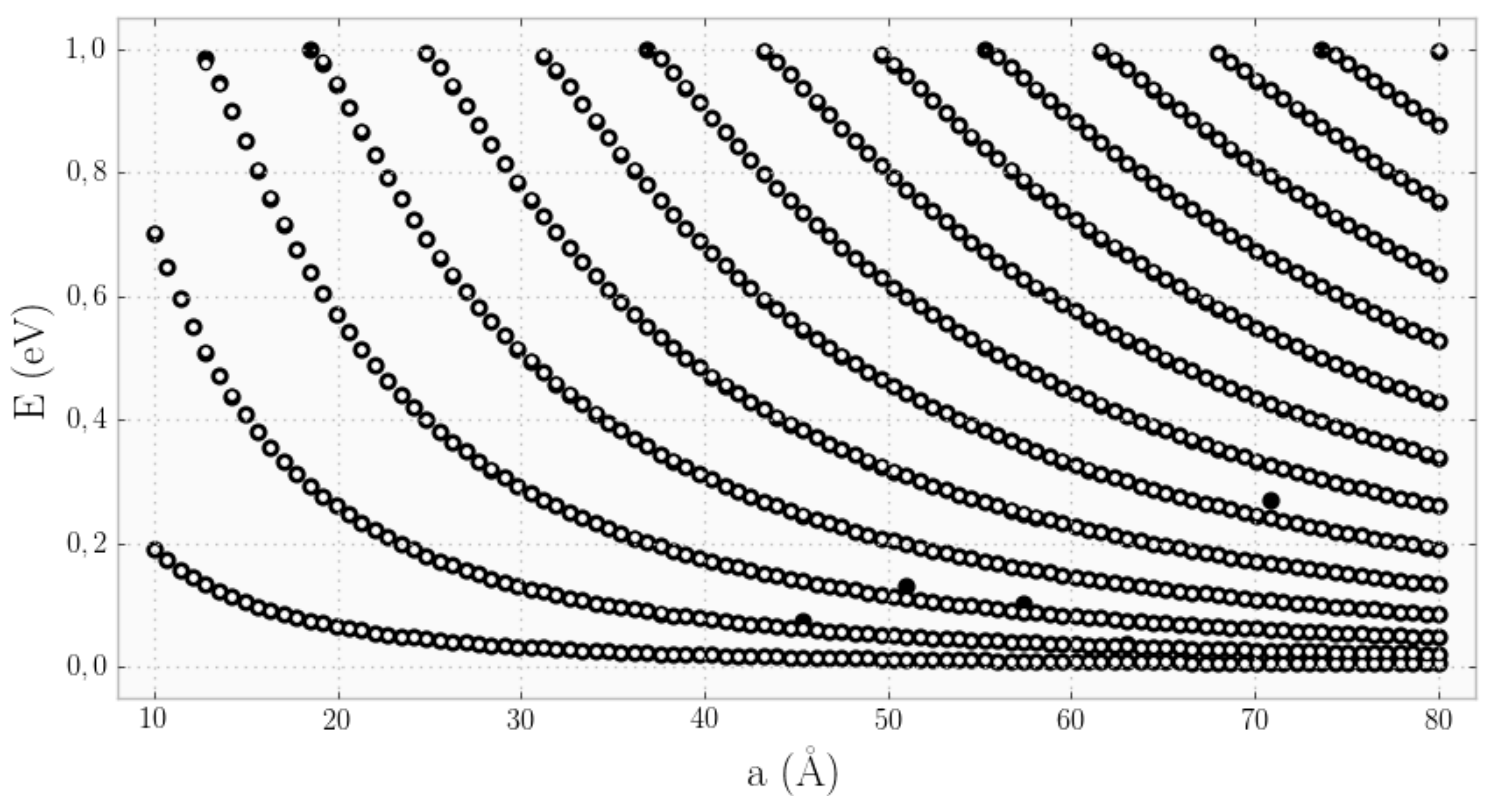

Figura 14: Autoenergias de estados possíveis para um elétron de massa $m_{\mathrm{e}}$ dentro de um poço quântico de altura $V_{\mathrm{b}}=1,0 \mathrm{eV}$ em função da largura, obtidos através do método Pseudo-Espectral em branco e através da solução das raízes das equações (5.25a) e (5.25b) em preto. Vale lembrar que alguns pontos pretos posicionados aleatoriamente entre as curvas se devem a divergência do algoritmo de Newton-Raphson utilizado. 


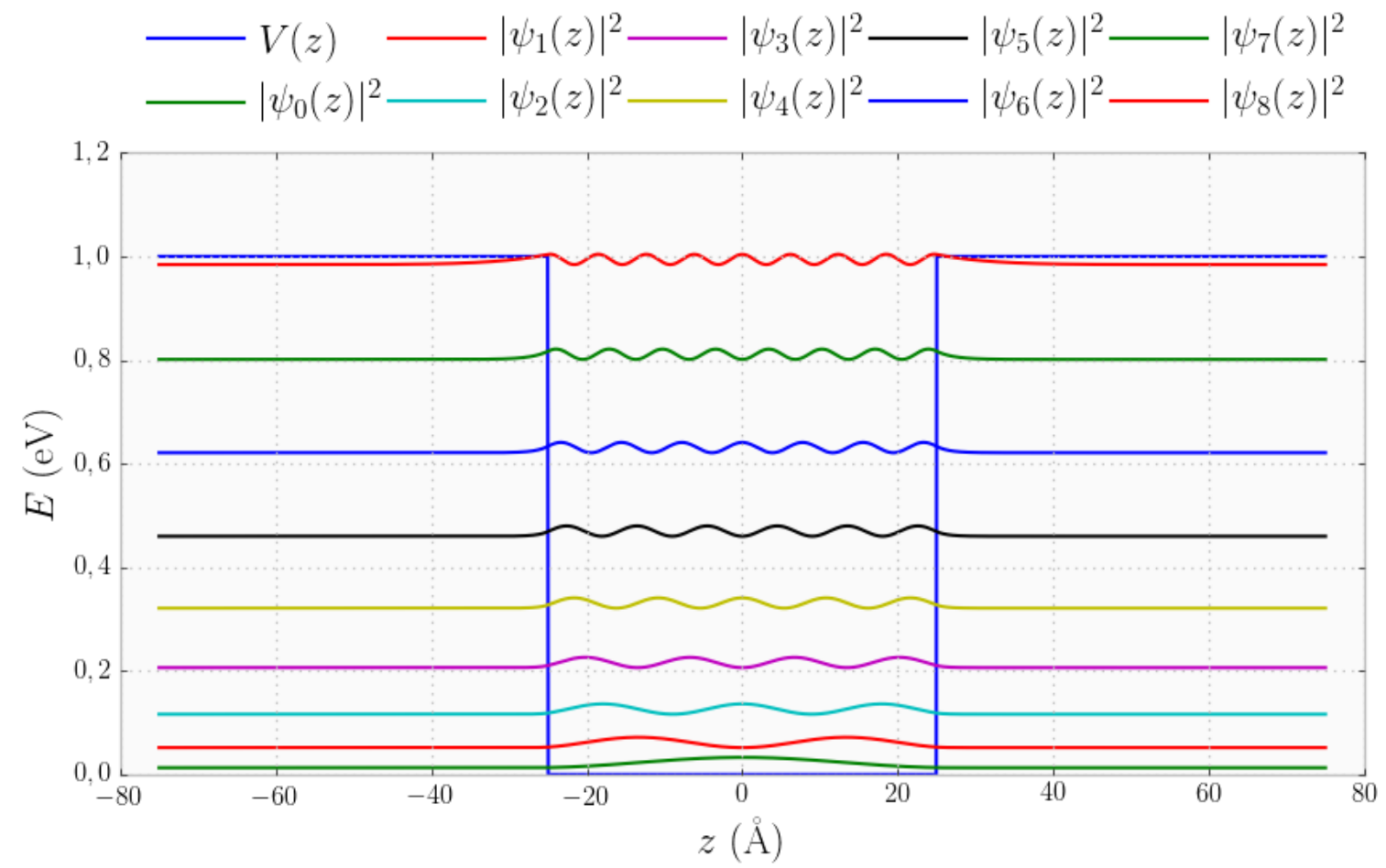

Figura 15: Autoestados possíveis para um elétron de massa $m_{\mathrm{e}}$ dentro de um poço quântico de altura $V_{\mathrm{b}}=1,0 \mathrm{eV}$ e largura 50,0 , obtidos através do método Pseudo-Espectral. As autoenergias deste poço podem ser conferidas na Tabela 4.

de quantos níveis precisamos calcular. Como o método de Newton-Raphson se mostrou um pouco instável nesta aplicação as vezes obtemos mais níveis do que deveríamos, ou seja, talvez nosso algoritmo disponível no Anexo A.2 calcule alguns desses níveis acima da altura do poço, mas que devem ser ignorados. É importante ressaltar que o método Pseudo-Espectral nos fornece tanto as autoenergias quanto os autoestados. Para ilustrar este fato, mostramos os autoestados de um poço de altura $1,0 \mathrm{eV}$ e largura $50,0 \AA$ na Figura 15.

Tabela 4: Autoenergias de um elétron de massa $m_{\mathrm{e}}$ dentro de poço quântico de altura 1,0 eV e largura $50,0 \AA$. Os valores analíticos foram obtidos através de solução das raízes das equações $(5.25 \mathrm{a})$ e $(5.25 \mathrm{~b})$ enquanto que os valores numéricos foram obtidos através do método Pseudo-Espectral.

\begin{tabular}{cccc}
\hline Autoenergia & Valor Analítico $(\mathrm{eV})$ & $\begin{array}{c}\text { Valor Numérico (eV) } \\
\text { (este trabalho) }\end{array}$ & Erro (\%) \\
\hline$E_{0}$ & 0,012937 & 0,012952 & 0,111857 \\
$E_{1}$ & 0,051700 & 0,051752 & 0,101488 \\
$E_{2}$ & 0,116129 & 0,116235 & 0,091498 \\
$E_{3}$ & 0,205930 & 0,206108 & 0,086792 \\
$E_{4}$ & 0,320603 & 0,320874 & 0,084524 \\
$E_{6}$ & 0,620440 & 0,620929 & 0,078811 \\
$E_{7}$ & 0,800413 & 0,801022 & 0,076099 \\
$E_{8}$ & 0,982859 & 0,984052 & 0,121385 \\
\hline
\end{tabular}


A concordância entre resultados numéricos e analíticos dos níveis de energia mostrados na Tabela 4 é muito boa, tanto em valores relativos (erros da ordem de 0,1\%) quanto em valores absolutos (erros da ordem de 0,05 meV para os primeiros dois níveis, que são em geral os mais importantes nos sistemas de interesse deste trabalho).

A precisão do método Pseudo-Espectral verificada nesta seção já nos permite utilizá-lo em outros sistemas, desde que a variação espacial da massa efetiva não seja importante, como já mencionado outras vezes. Na próxima seção estudamos (como forma de verificar nosso domínio sobre a técnica) um sistema reportado na literatura onde os autores utilizaram o próprio método Pseudo-Espectral para obter os autoestados e autovalores de um potencial de interessa da área de Química Quântica.

\subsection{Cálculo de Níveis de Energia: Poço Duplo Assimétrico}

Nesta seção testamos o método Pseudo-Espectral para obtenção das autofunções e autoenergias de um problema com solução analítica e numérica reportada na literatura. Utilizamos a técnica de evolução em tempo imaginário para obter o primeiro estado e ortogonalização de Gram-Schmidt para obter os demais.

Este potencial é de grande interesse para o estudo de vibrações e espalhamento em moléculas hidrogenóides. Apesar de não existir uma solução completamente analítica, Zhirnov e Turev [43] propuseram uma aproximação bastante eficiente que possibilitou a obtenção dos autoestados e autovalores em 1979. Em 1982, Feit, Fleck e Steiger [1] utilizaram um método Pseudo-Espectral para obter autoestados e autovalores deste potencial, em grande acordo com a solução proposta for Zhirnov e Turev [43]. Nestes cálculos utilizamos um maior número de pontos (2048) na malha espacial e um passo de tempo menor $\left(1 \times 10^{-20} \mathrm{~s}\right)$ do que aqueles usados $\left(512\right.$ e $\left.1,386 \times 10^{-18} \mathrm{~s}\right)$ pelos autores do estudo numérico de Feit e colaboradores, [1] com o objetivo de verificar se era possível obter uma precisão ainda melhor, tendo como alvo os valores reportados pelos autores que propuseram a aproximação analítica.

A forma do poço duplo assimétrico investigada é dada por:

$$
V(x)=-k_{2} x^{2}+k_{3} x^{3}+k_{4} x^{4}
$$

onde $k_{2}=+7,0, k_{3}=+0,5$ e $k_{4}=+0,1$. No caso deste potencial, os autores sugerem a utilização da massa efetiva do elétron como sendo $m_{\mathrm{e}}^{*}=0,5$ (em unidades atômicas). O perfil deste potencial é mostrado na Figura 16 juntamente com seus autoestados para os primeiros 9 níveis de energia.

Na Tabela 5 é feita a comparação entre os resultados obtidos utilizando o método Pseudo-Espectral e aqueles obtidos pelos autores Feit, Fleck e Steiger [1]. Vemos que 

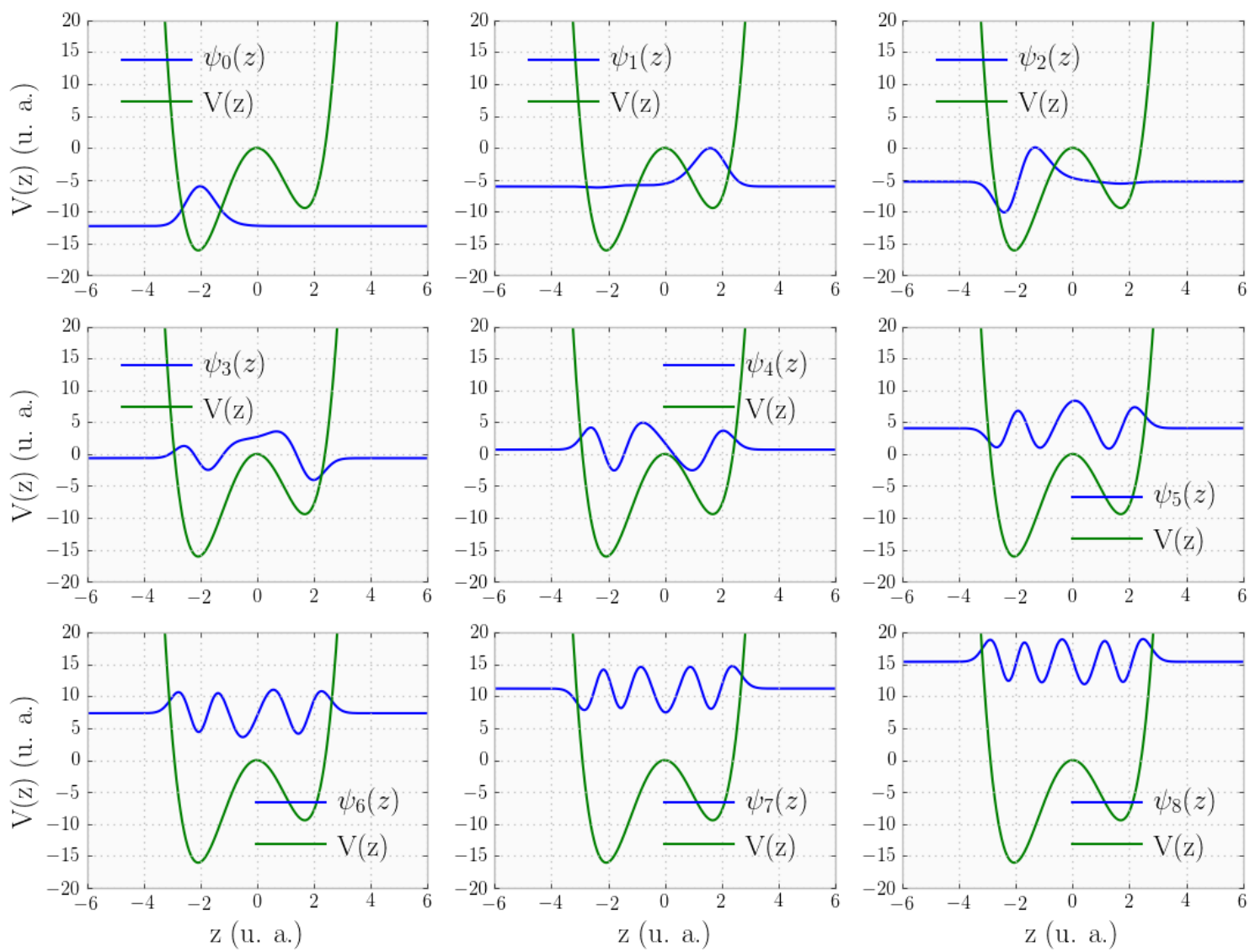

Figura 16: Nove primeiras autofunções para um elétron com massa efetiva $m_{\mathrm{e}}^{*}=0,5$ (unidades atômicas) dentro de um poço duplo assimétrico obtidas pelo método Pseudo-Espectral. As autofunções estão em grande acordo com os resultados analíticos obtidos por Zhirnov e Turev [43].

nossos resultados se aproximam mais dos resultados de Zhirnov e Turev [43] para a maioria dos níveis de energia, sendo a única exceção o nível 3.

Tabela 5: Comparação entre o método Pseudo-Espectral e resultados numéricos obtidos por Feit, Fleck e Steiger [1] e valores analíticos obtidos por Zhirnov e Turev [43]. Todos os valores estão em unidades atômicas.

\begin{tabular}{cccc}
\hline $\mathrm{n}$ & Ref.[1] & Ref.[43] & $\begin{array}{c}\text { Pseudo-Espectral } \\
\text { (este trabalho) }\end{array}$ \\
\hline 0 & $-12,258438$ & $-12,258799$ & $-12,258812$ \\
1 & $-6,045418$ & $-6,045687$ & $-6,045697$ \\
2 & $-5,286089$ & $-5,286859$ & $-5,286911$ \\
3 & $-0,646627$ & $-0,646541$ & $-0,646958$ \\
4 & 0,691204 & 0,690390 & 0,690299 \\
5 & 4,053229 & 4,052355 & 4,052223 \\
6 & 7,368937 & 7,367668 & 7,367444 \\
7 & 11,235521 & 11,233904 & 11,233544 \\
8 & 15,431918 & 15,429861 & 15,429334 \\
\hline
\end{tabular}




\subsection{Fotocorrente e Absorção em Poço Quântico de GaAs com Bar- reiras de $\mathrm{AlGaAs}$}

Nesta seção calculamos a fotocorrente e o espectro de absorção de uma estrutura proposta em 2011 por Degani et al [6] com o propósito de reduzir a corrente de escuro ${ }^{2}$ em fotodetectores.

Tanto para o cálculo da fotocorrente quanto para o cálculo do espectro de absorção, é necessário conhecer os estados ligados do sistema. Nesta seção decidimos testar o método da Iteração Inversa para obter as autofunções e autoenergias. Este método demonstrou-se mais eficiente nesta tarefa de calcular autofunções do que o método Pseudo-Espectral. Entretanto, o método da Iteração Inversa só atinge sua performance máxima quando as autoenergias são conhecidas, mesmo que aproximadamente. Por esta razão seguimos utilizando os dois em paralelo, verificando grande concordância entre os resultados obtidos por ambos. Identificamos que a melhor estratégia é utilizar o método Pseudo-Espectral para obter as autoenergias pelo menos uma vez, depois disso sendo possível utilizar estas autoenergias como chutes iniciais para o método da Iteração Inversa, pois estes valores seguem úteis mesmo que haja alguma leve alteração no sistema, como a aplicação de uma diferença de potencial fraca, como veremos a seguir.

Na Figura 17 é mostrada a estrutura proposta pelos referidos autores, bem como o perfil de potencial resultante. É importante observar que na banda de condução existe um poço cercado por barreiras. Os estados ligados são mostrados na Figura 18, onde é possível ver que além de um estado dentro do poço existem mais dois estados confinados pelas barreiras, mas que possuem energias acima do topo do poço.

Uma vez conhecidas as autofunções e autoenergias, utilizamos o método PseudoEspectral para evoluir estas autofunções no tempo. Apesar de o método Pseudo-Espectral não permitir que seja considerada a variação espacial da massa efetiva ${ }^{3}$, os autores sugerem que é uma boa aproximação tomar a massa efetiva do elétron como sendo a massa efetiva do GaAs ao longo de toda a estrutura.[6]

A fotocorrente ocorre quando fótons são capazes de excitar elétrons confinados em poços quânticos. Para simular a incidência de fótons, adicionamos no hamiltoniano um campo elétrico variável de frequência angular $\omega$ e consequentemente energia $E=\hbar \omega$. Uma outra questão é que uma vez excitado por um fóton, um elétron não tem direção preferencial no potencial da Figura 17. Uma solução para isso é adicionar uma diferença de potencial na estrutura, criando um viés para que o elétron se desloque preferencialmente para um dos lados e assim possamos estimar uma fotocorrente líquida não nula.

2 Elétrons confinados em poços quânticos podem eventualmente ser excitados através da interação com fônons da rede.

3 Dentro da aproximação da massa efetiva, como existem regiões do material com diferentes concentrações de $\mathrm{Al}$, a massa efetiva do elétron tende a ser diferente em cada uma delas. 


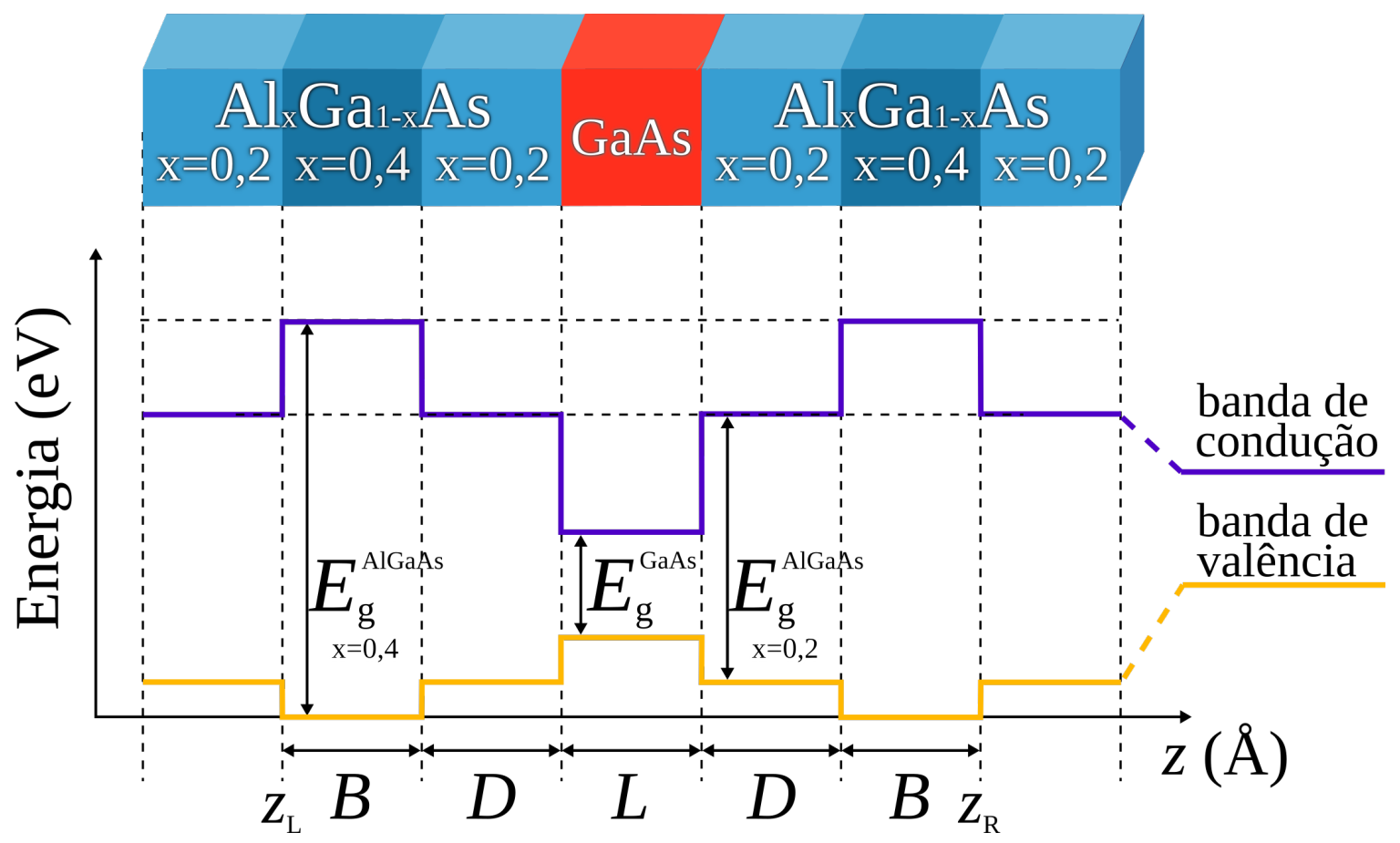

Figura 17: Estrutura semicondutora proposta por Degani et al [6] com a finalidade de intensificar a fotocorrente para um determinado comprimento de onda ao mesmo tempo em que reduz a corrente de escuro. Na região $L$ o material é somente GaAs. Nas regiões $B$ o material é $\mathrm{Al}_{0,4} \mathrm{Ga}_{0,6} \mathrm{As}$. Já nas regiões $D$ e nas laterais o material é $\mathrm{Al}_{0,2} \mathrm{Ga}_{0,8} \mathrm{As}$.

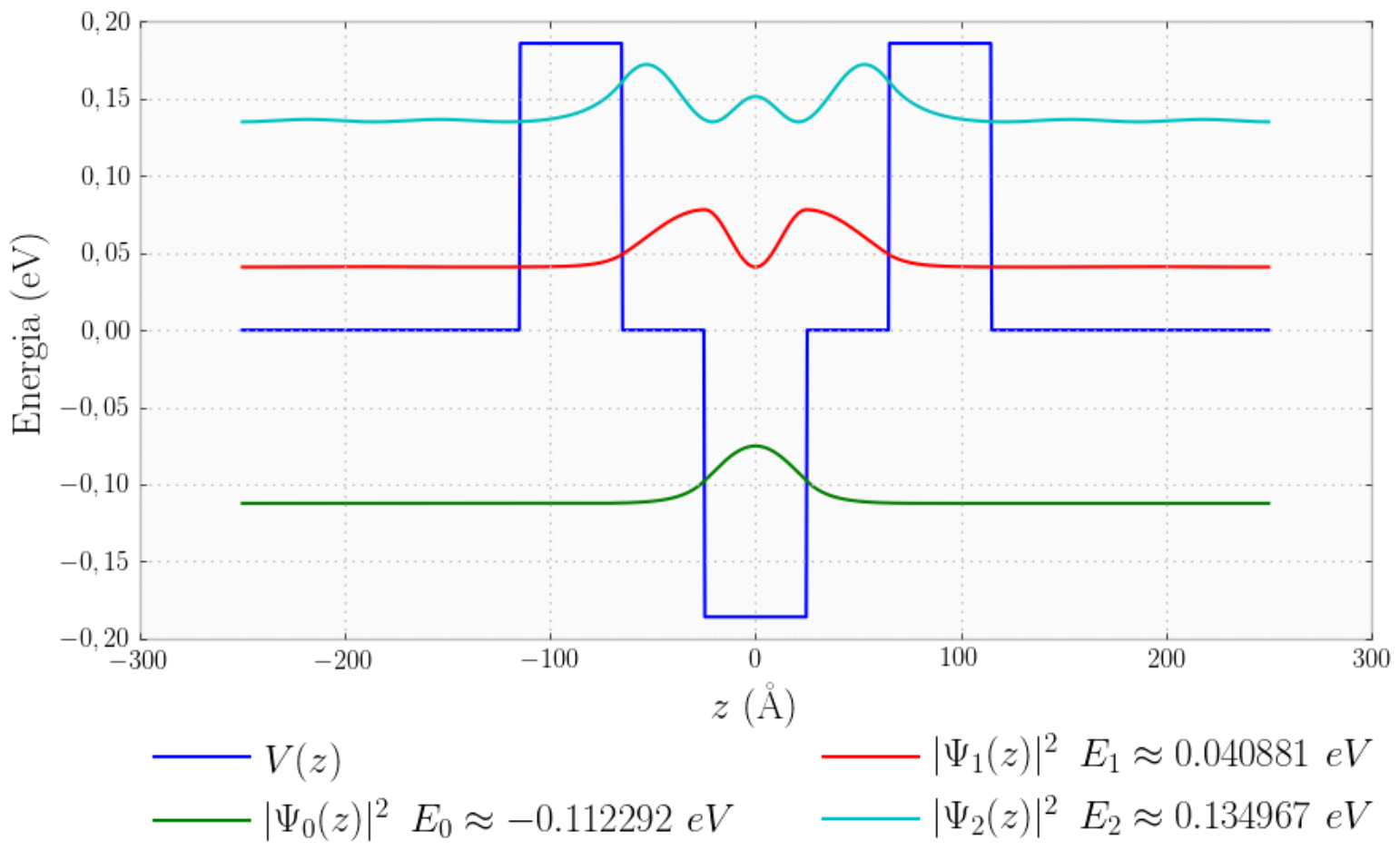

Figura 18: Autofunções e autoenergias de um potencial como o da Figura 17 obtidos através do método da Iteração Inversa. 
Na prática, uma camada de material fortemente dopado é posicionada ao redor da estrutura, de modo que nesta região o potencial é praticamente constante, como em um metal.[7] O hamiltoniano completo do sistema, levando em consideração a diferença de potencial e o campo elétrico variável assume então a forma [6]

$$
\mathbf{H}=-\frac{\hbar^{2}}{2} \frac{\partial}{\partial z} \frac{1}{m(z)} \frac{\partial}{\partial z}+V(z)- \begin{cases}e z_{L}\left(F_{\text {est }}+F_{\text {osc }} \sin (\omega t)\right) & \text { se } z \leq z_{L} \\ e z\left(F_{\text {est }}+F_{\text {osc }} \sin (\omega t)\right) & \text { se } z_{L} \leq z \leq z_{R} \\ e z_{R}\left(F_{\text {est }}+F_{o s c} \sin (\omega t)\right) & \text { se } z \geq z_{R}\end{cases}
$$

onde $F_{\text {est }}$ é a intensidade do campo estático devido à diferença de potencial, $F_{o s c}$ é a intensidade do campo elétrico oscilante, $z_{L}$ e $z_{R}$ são os pontos em $z$ imediatamente a esquerda e a direita das barreiras, respectivamente, como indicados na Figura 17.

Como a diferença de potencial afeta o dispositivo de forma constante ao longo de todo o cálculo, obtemos os autovalores e autovetores já em sua presença, ou seja, com $F_{\text {est }} \neq 0$ e $F_{\text {osc }}=0$, assim como fazem os referidos autores. [6]

Supomos a existência de um elétron no estado fundamental e no instante $t=0$ fazemos $F_{\text {osc }} \neq 0$. Apesar de termos optado por utilizar o método Pseudo-Espectral, a evolução temporal pode ser feita com os métodos de Runge-Kutta ou Crank-Nicolson, sendo estes dois fortemente recomendados no caso em que a massa efetiva não seja tomada como constante ao longo de toda a estrutura.

Sabemos que os métodos de Runge-Kutta e Crank-Nicolson podem fazer uso do esquema indicado na Eq. (4.31) para levar em conta a variação espacial da massa efetiva ao longo da heteroestrutura. Entretanto, o método de Runge-Kutta envolve cinco produtos de matrizes $N \times N$ enquanto que o método de Crank-Nicolson necessita de uma inversão de matriz e mais dois produtos, fazendo deles métodos bastante custosos computacionalmente. Apesar de não permitir a consideração da variação espacial da massa efetiva, o método Pseudo-Espectral é substancialmente mais rápido.

Como utilizamos o operador de evolução temporal do método Pseudo-Espectral, precisamos atenuar partes da função de onda que atinjam alguma das extremidades do sistema. Na literatura encontramos algumas referências ao uso do potencial imaginário, ou potencial óptico, capaz de absorver a função de onda incidente sobre ele.[3, 6, 44] Entretanto, obtivemos melhores resultados com um procedimento mais simples, onde a cada passo de tempo, multiplicamos a função de onda por um potencial absorvedor, conforme mostramos na Figura 19. No alto da figura mostramos o gráfico do potencial absorvedor $A(z)$. No gráfico central a função $F(z)=\sin (z)$. No gráfico inferior mostramos o produto de $A(z) \times F(z)$ onde podemos observar a eficiente atenuação da função $F(z)$ nas extremidades do sistema pelo potencial absorvedor. 

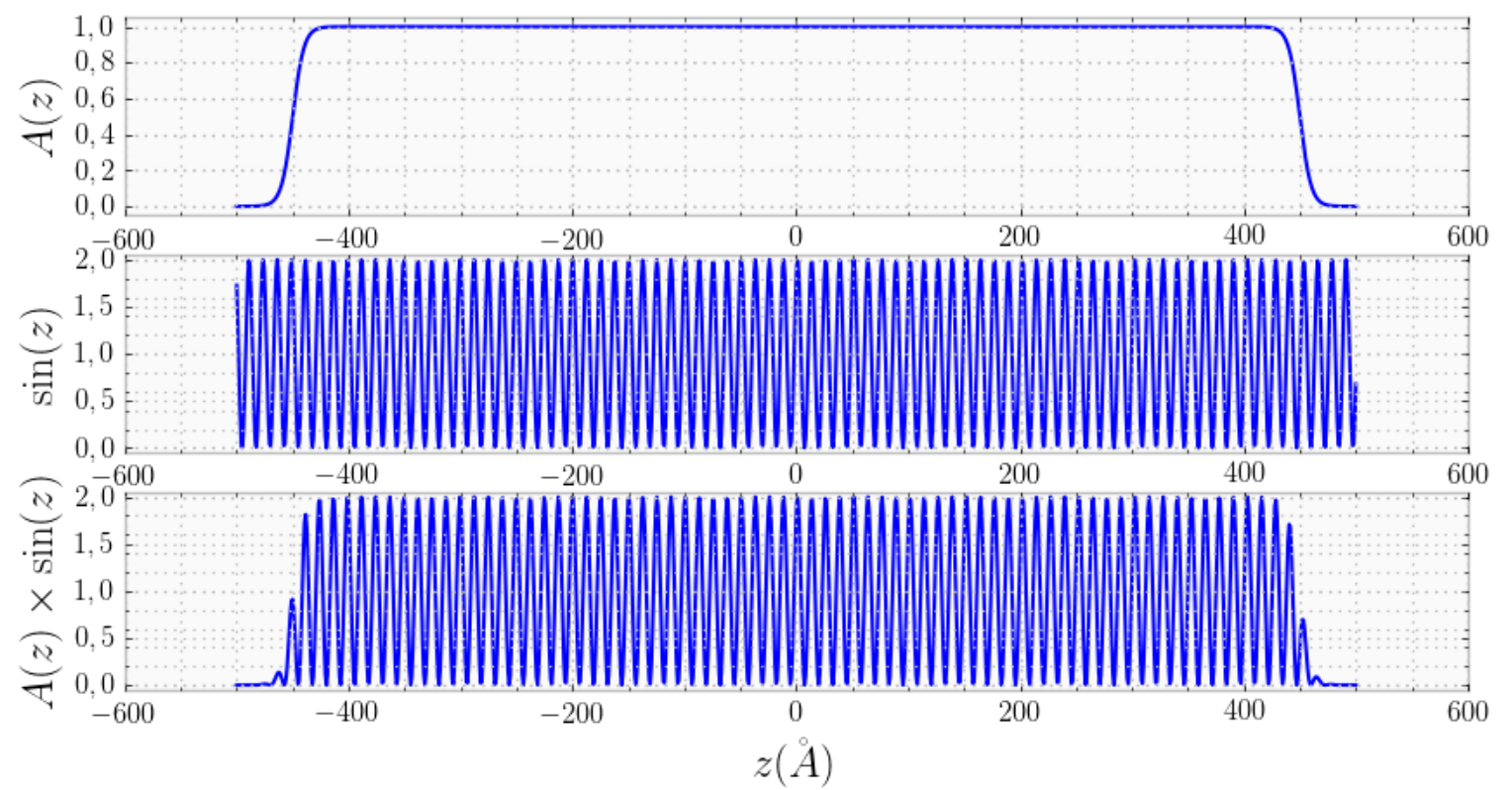

Figura 19: Exemplo de como o potencial absorvedor $A(z)$ da Eq. (5.28) age sobre uma função nas proximidades das extremidades do sistema. No caso é mostrada apenas sua ação sobre uma função $\sin (z / 2+1)$.

A equação do potencial absorvedor é dada por

$$
A(z)= \begin{cases}\frac{1}{1+\exp (-(z+0,45 L) / 5)} & \text { se } z \leq 0 \\ \frac{1}{1+\exp (-(0,45 L-z) / 5)} & \text { se } z>0\end{cases}
$$

onde $L$ é o tamanho to sistema. Os valores 0,45 e 5 foram obtidos por tentativa e erro, com a intenção de comprometer o mínimo possível a malha espacial e ainda assim garantir que a função de onda vá a zero nas extremidades.

A fotocorrente mostrada na Figura 20 é calculada de acordo com a Eq. (2.35) por um período de $10^{-12} \mathrm{~s}$, com incrementos de $10^{-17} \mathrm{~s}$, ou seja, são realizados $10^{5}$ aplicações do operador de evolução temporal sobre o estado inicial. O algoritmo utilizado pode ser conferido no Anexo A.5.

A comparação do espectro da Figura 20 com aquele mostrado na Figura 21 (retirado do trabalho de Degani e colaboradores [6]) mostra que obtivemos um espectro de fotocorrente muito semelhante, especialmente no que diz respeito à posição dos máximos.

Os níveis de energia (para $F_{\text {est }}=0$ ) deste sistema (obtidos tanto por nós quanto por Degani et al) são $E_{0}=-112,292 \mathrm{meV}, E_{1}=40,881 \mathrm{meV}$ e $E_{2}=134,967 \mathrm{meV}$, o que significa que o primeiro máximo deveria ocorrer em $E_{1}-E_{0} \approx 153 \mathrm{meV}$, enquanto que o segundo deveria ocorrer em $E_{2}-E_{0} \approx 247 \mathrm{meV}$. Isso significa que tanto o nosso procedimento quanto o de Degani et al reproduzem a energia esperado do primeiro máximo, mas falham na localização do segundo.

Diferentemente do que é feito para a fotocorrente, no caso do espectro de absorção, 


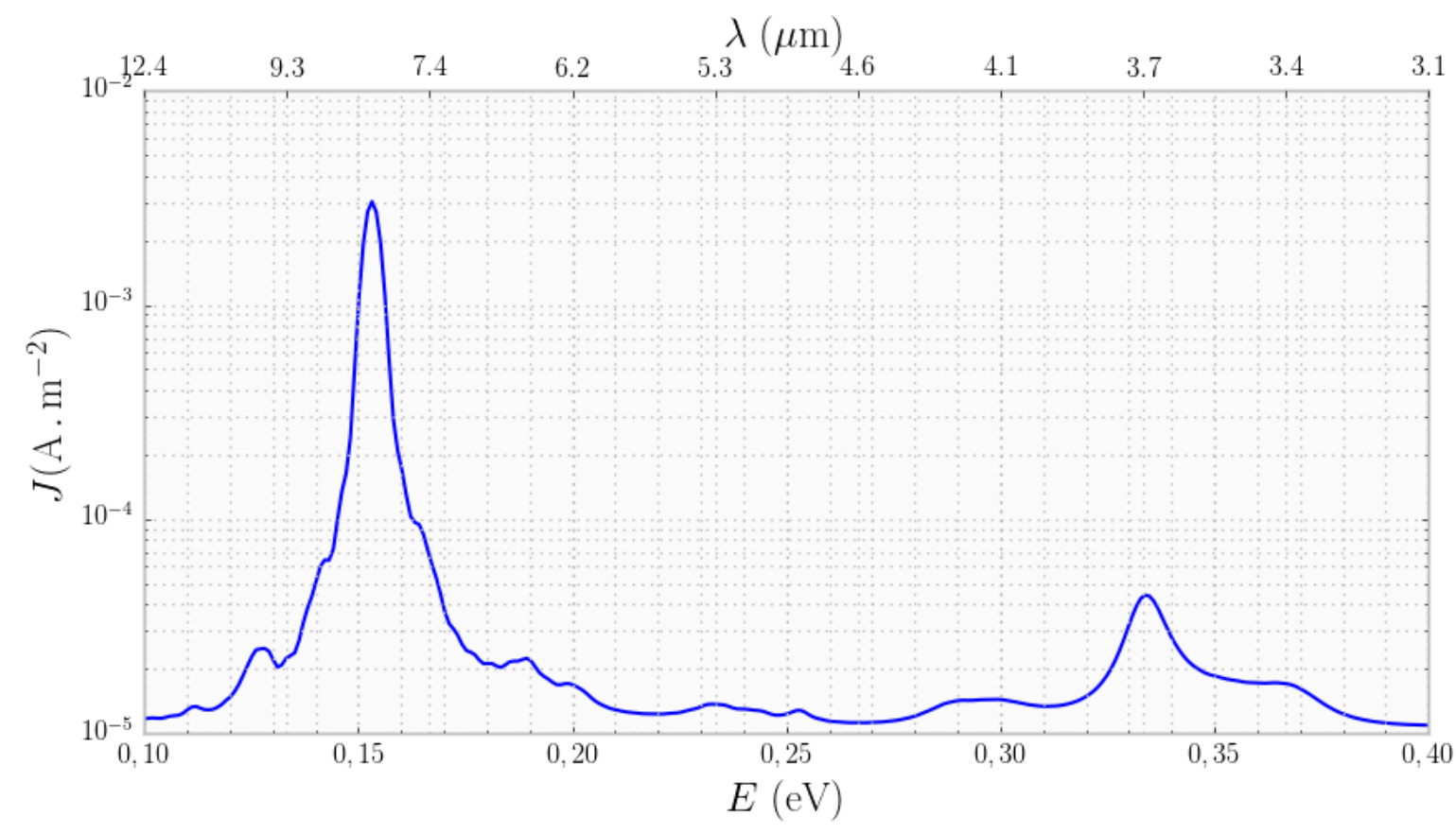

Figura 20: Fotocorrente calculada neste trabalho para um potencial como o indicado na Figura 17.

os referidos autores sugerem não utilizar a diferença de potencial estática para enviezar o momento das partes da função de onda que eventualmente venham a tunelar por uma das barreiras. Para o cálculo do espectro de absorção, utilizamos a Eq. (2.64). Neste caso, o potencial absorvedor da Eq. (5.28) com os valores 0,45 e 5 não foi suficiente para reduzir o ruído, como é possível observar na Figura 22. Apesar de o primeiro pico de absorção ocorrer precisamente quando a frequência do campo elétrico equivale (em energia) à energia de transição do estado fundamental para o primeiro estado excitado $(\sim 153 \mathrm{meV})$, o segundo pico que deveria ocorrer em aproximadamente $\sim 247 \mathrm{meV}$ ocorre acima de $300 \mathrm{meV}$. Os
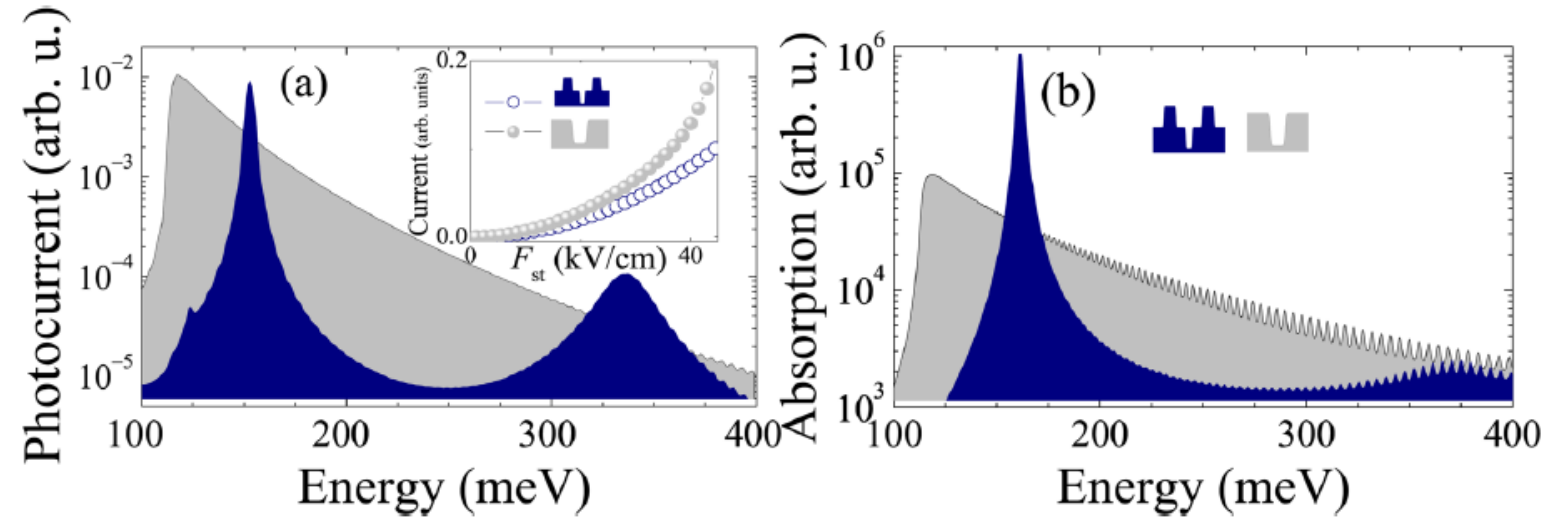

Figura 21: Fotocorrente e espectro de absorção obtidos na referência [6]. As curvas em azul são atribuídas à estrutura do poço de dupla barreira que buscamos reproduzir, enquanto que as curvas em cinza são atribuídas à um poço quântico simples. Na figura (a) é mostrada a fotocorrente que possui um máximo em aproximadamente $153 \mathrm{meV}$ e outro em aproximadamente $333 \mathrm{meV}$. Na figura (b) é mostrado o espectro de absorção com um único máximo em aproximadamente $153 \mathrm{meV}$ e uma região ruidosa acima de $350 \mathrm{meV}$. 


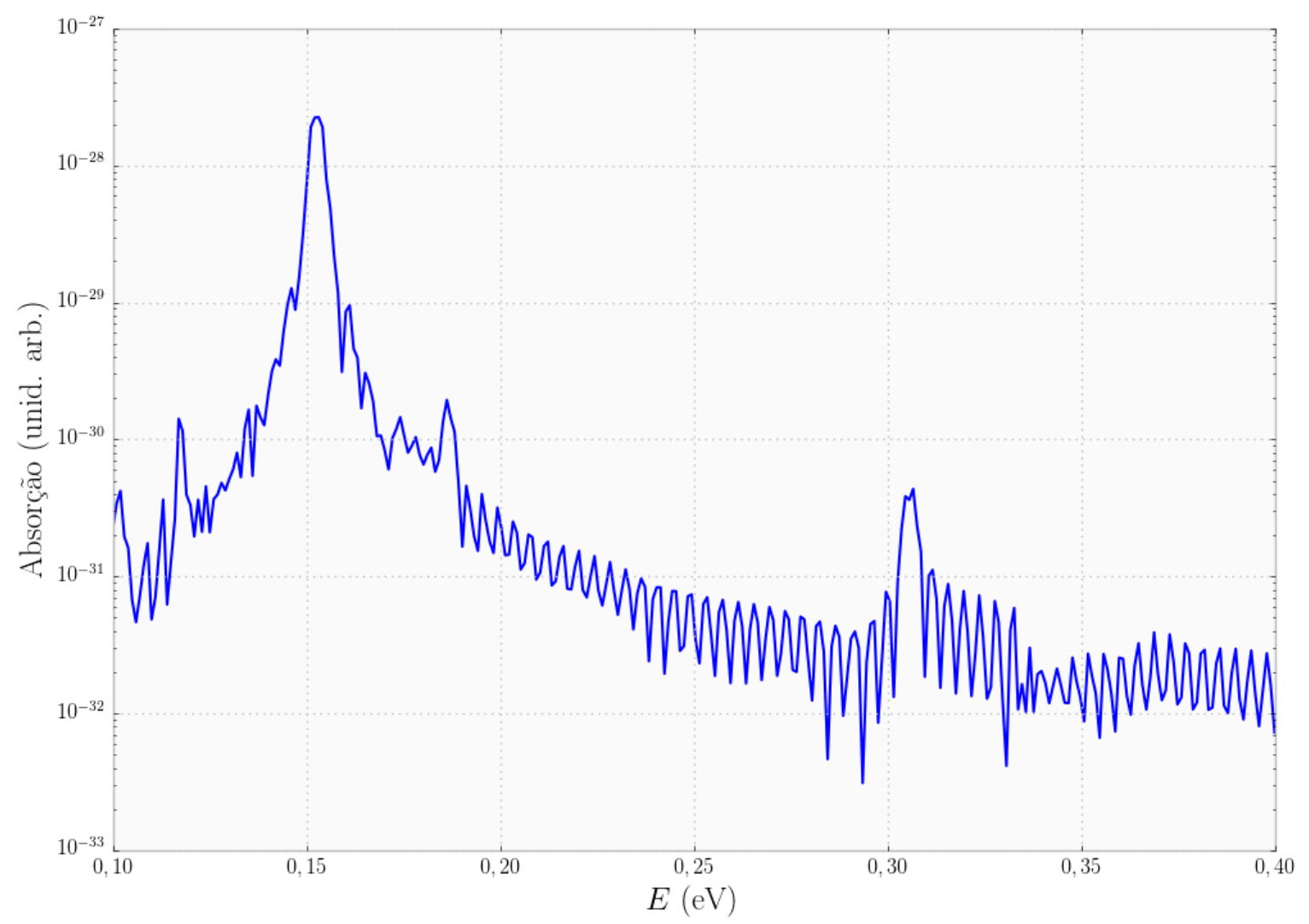

Figura 22: Espectro de absorção calculado neste trabalho para um potencial como o indicado na Figura 17, que reproduz parcialmente o resultado publicado na referência [6].

referidos autores não indicam, para a absorção, outro máximo além daquele referente à transição entre o estado fundamental e o primeiro estado excitado.

Acreditamos que a redução do forte ruído observado na Figura 22 bem como o posicionamento errático do segundo pico possam ser corrigidos trabalhando melhor o potencial absorvedor. Em algumas tentativas obtivemos máximos nos lugares corretos, entretanto outros máximos ainda prevalecem, como na Figura 23, onde são indicados os máximos esperados e os inesperados.

Atribuímos este ruído ao mal ajuste do potencial absorvedor porque uma parcela da função de onda, que eventualmente tunela por uma das barreiras, não está sujeita a uma diferença de potencial que orienta o seu movimento em direção aos limites do sistema, como no caso da fotocorrente. O que de fato e que foi observado durante a evolução da função no tempo é que esta parcela que tunela fica avançando e regredindo em uma região do espaço (sob ação do campo elétrico oscilante), sendo contabilizada mais de uma vez a sua contribuição para a absorção. 


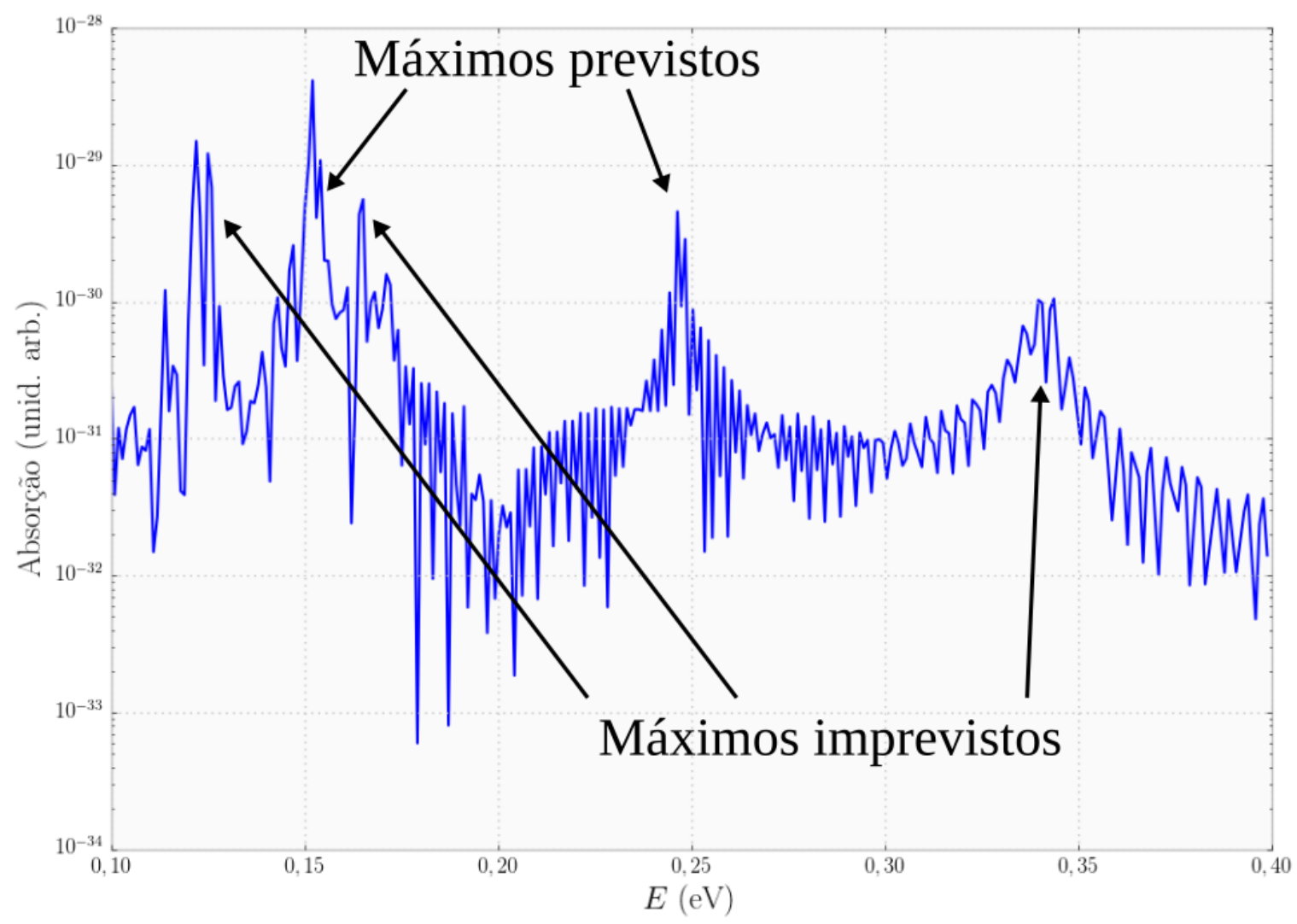

Figura 23: Espectro de absorção calculado neste trabalho para um potencial como o indicado na Figura 17, onde são observados máximos nas posições corretas $(\sim 153 \mathrm{meV}$ e $\sim 247 \mathrm{meV})$, mas também alguns máximos imprevistos, atualmente atribuídos ao mal ajuste do potencial absorvedor. 


\section{Efeitos da Tensão e Segregação do Índio (In) em Poços Quânticos de InGaAs/GaAs}

O poço quântico de InGaAs/GaAs é obtido intercalando-se camadas de InGaAs entre camadas de GaAs. Além de sua importância tecnológica, este sistema representa um excelente teste para os procedimentos numéricos que desenvolvemos neste trabalho dada a forma particular do perfil de potencial da estrutura resultante. Para o correto modelamento da estrutura eletrônica destes poços devemos considerar que o crescimento de InGaAs sobre GaAs ocorre sob tensão (efeito este que altera a energia do gap do InGaAs) e sob o fenômeno da segregação de índio (que modifica o perfil de potencial retangular do poço).

Neste capítulo, apresentamos a forma como os efeitos da tensão e da segregação alteram o perfil de potencial de uma heteroestrutura semicondutora e realizamos os cálculos dos níveis de energia para uma amostra crescida no MBE do LNMS do IF-USP, contendo 4 poços de InGaAs/GaAs. Os resultados dos cálculos são comparados com medidas de fotoluminescência (PL) em baixa temperatura.

\subsection{Influência da Tensão Elástica}

Quando um filme epitaxial (epicamada) e o substrato sobre o qual ele é crescido possuem a mesma estrutura cristalina e parâmetros de rede muito parecidos (caso do AlGaAs sobre GaAs) ou idêntico (GaAs sobre GaAs), o crescimento ocorre de maneira coerente, isto é, o material depositado adota o mesmo parâmetro de rede que o do substrato, e o filme cresce camada por camada. No entanto, quando o material evaporado tem um parâmetro de rede diferente (caso do InGaAs sobre GaAs), a camada depositada é tensionada e permanece epitaxial (com o mesmo parâmetro de rede que o substrato) até alcançar uma espessura crítica a partir da qual deformações estruturais (deslocações) são introduzidas na epicamada para que ela possa adotar seu parâmetro de rede natural. $\mathrm{O}$ crescimento torna-se então incoerente.

As ligas ternárias de InGaAs têm parâmetros de rede maiores do que o parâmetro de rede do GaAs. Desta forma, para que o crescimento ocorra de forma coerente, é necessário que algum tipo de deformação obrigue o InGaAs a adotar o parâmetro de rede do GaAs. De fato, a camada epitaxial de InGaAs é acomodada pela deformação elástica da camada inteira, que adota o parâmetro de rede lateral do substrato mas aumenta o parâmetro de rede vertical, como mostrado na Figura 24. Este processo de ajuste de pa- 
(a)

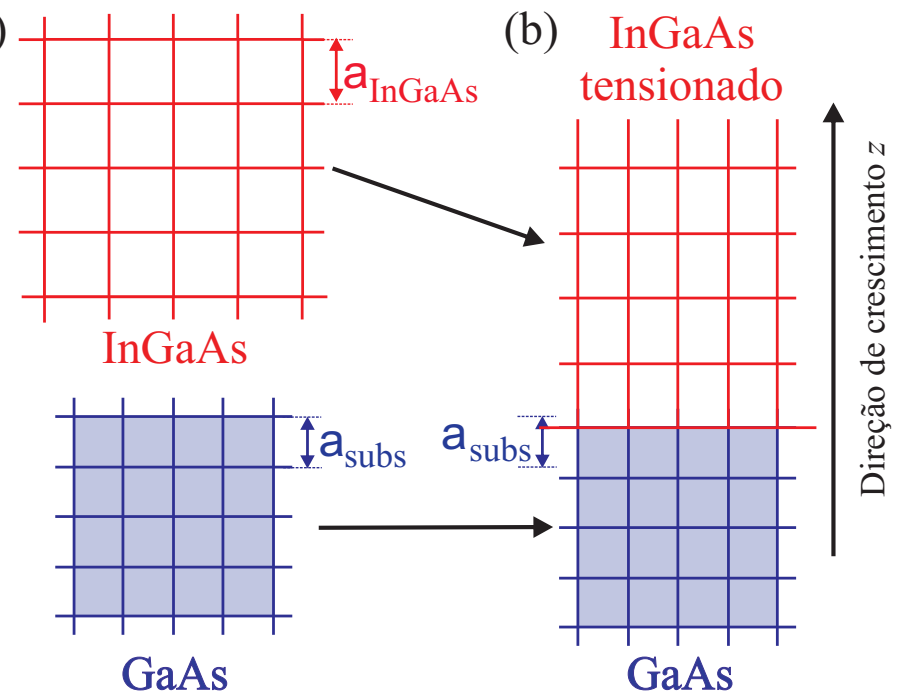

Figura 24: Ilustração de uma camada de InGaAs e de um substrato de GaAs: (a) Quando os dois materiais estão separados, cada um possui um parâmetro de rede próprio. (b) Ao crescer InGaAs sobre o substrato de GaAs, o InGaAs adota o mesmo parâmetro de rede lateral que o GaAs e aumenta o seu parâmetro de rede vertical, gerando tensão na camada depositada.

râmetro de rede introduz tensão biaxial nas camadas de InGaAs, que altera a energia do gap do material sob tensão.

Se não consideramos a influência da tensão elástica, as alturas dos potenciais de confinamento para os elétrons, $\Delta E_{\mathrm{c}}$, e para os buracos, $\Delta E_{\mathrm{v}}$, são dadas por:

$$
\begin{aligned}
& \Delta E_{\mathrm{v}}(x)=V B O^{\mathrm{GaAs}}-V B O^{\mathrm{InGaAs}}(x), \\
& \Delta E_{\mathrm{c}}(x)=E_{\mathrm{g}}^{\mathrm{GaAs}}-E_{\mathrm{g}}^{\mathrm{InGaAs}}(x)-\Delta E_{\mathrm{v}}(x),
\end{aligned}
$$

seguindo a terminologia introduzida na Seção 3.2 e esquematizada novamente na Figura 25. Na Eq. (6.1) e no restante deste capítulo, $x$ é um número no intervalo $0 \leq x \leq 1$ que descreve a porcentagem de índio na liga ternária de $\operatorname{In}_{x} \mathrm{Ga}_{1-x}$ As. Este parâmetro permite que tenhamos expressões válidas ao longo de toda a estrutura, uma vez que fora da região central, temos $x=0$, ou seja, $\mathrm{In}_{0} \mathrm{Ga}_{1} \mathrm{As}=\mathrm{GaAs}$.

A influência da tensão é considerada fazendo-se uma correção na altura dos potenciais (tanto na banda de condução quanto na banda de valência). Em especial no topo da banda de valência, surgem bandas bastante próximas, chamadas de banda de buracos leves (lh, do inglês light holes) e banda de buracos pesados (hh, do inglês heavy holes).[11] Estas nomenclaturas se devem às curvaturas dessas bandas na região em que $\mathbf{k}=0$ nos gráficos de dispersão energia-momento, levando-se em conta a aproximação da massa efetiva. Para fins de cálculo de energias de recombinação observadas por fotoluminescência, que abordamos nas próximas seções, devemos nos preocupar somente com a banda de buracos pesados.[11, 24, 34] O potencial de confinamento para buracos pesados será dado por: $[11,34,48]$

$$
\Delta E_{\mathrm{v}}^{\mathrm{hh}}(x)=\Delta E_{\mathrm{v}}(x)+a_{\mathrm{v}}(x)\left(\epsilon_{x x}+\epsilon_{y y}+\epsilon_{z z}\right)-\frac{b(x)}{2}\left(\epsilon_{x x}+\epsilon_{y y}-2 \epsilon_{z z}\right),
$$




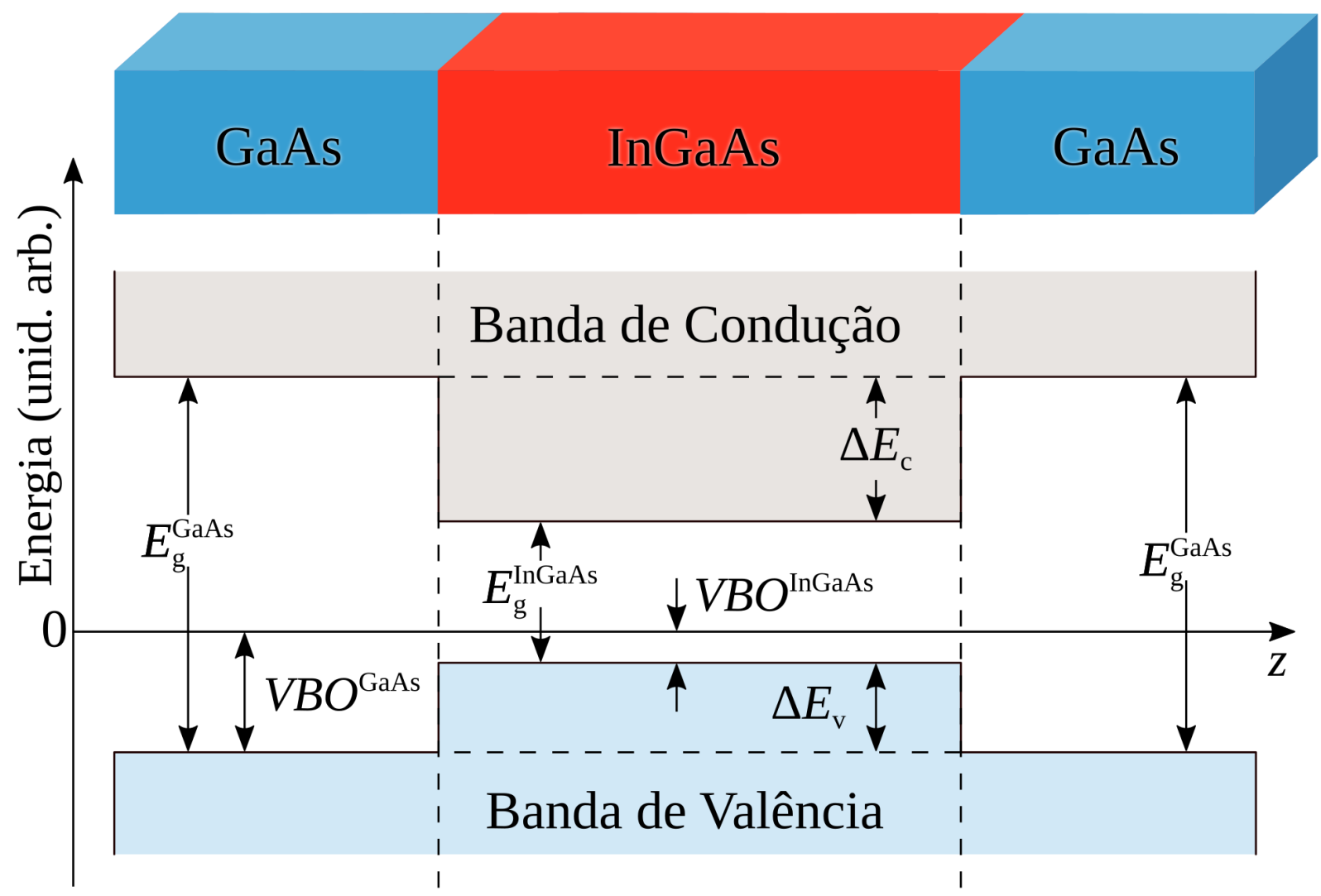

Figura 25: Alinhamento das bandas de valência e condução como resultado das diferenças de gap e de $V B O$ das ligas GaAs e InGaAs.

sendo $a_{\mathrm{v}}(x)$ o potencial de deformação hidrostático da banda de valência e $b(x)$ o potencial de deformação da distorção tetragonal. Os elementos do tensor de deformação são dados por:

$$
\begin{aligned}
& \epsilon_{x x}(x)=\epsilon_{y y}(x)=\frac{a_{\mathrm{GaAs}}-a_{\mathrm{InGaAs}}(x)}{a_{\mathrm{InGaAs}}(x)} \\
& \epsilon_{z z}(x)=-\frac{2 C_{12}(x)}{C_{11}(x)} \epsilon_{x x}(x)
\end{aligned}
$$

onde as constantes elásticas $C_{11}(x)$ e $C_{12}(x)$ e o parâmetro de rede $a_{\text {InGaAs }}$ do InGaAs dependem da composição $x$ da liga ternária, e $a_{\mathrm{GaAs}}$ é o parâmetro de rede do GaAs.

O potencial de confinamento para os elétrons na banda de condução será dado por: $[11,34,48]$

$$
\Delta E_{\mathrm{c}}=E_{\mathrm{g}}^{\mathrm{GaAs}}-E_{\mathrm{g}}^{\mathrm{InGaAs}}(x)-\Delta E_{\mathrm{v}}^{\mathrm{hh}}(x)+a_{\mathrm{c}}(x)\left(\epsilon_{x x}+\epsilon_{y y}+\epsilon_{z z}\right)
$$

sendo $a_{\mathrm{c}}(x)$ o potencial de deformação hidrostática.

\subsection{Parâmetros e Ilustração das Correções Induzidas pela Tensão}

Os parâmetros físicos que descrevem os materiais semicondutores binários, em geral, são conhecidos (calculados ou determinados experimentalmente). Isso não é verdade 
para as ligas ternárias, quaternárias, etc. Então, o procedimento utilizado para a determinação dos parâmetros das ligas ternárias é a interpolação linear entre os parâmetros dos materiais binários, também conhecida como Lei de Vegard.[7, 11, 48] Por exemplo, se um material é constituído de uma liga ternária que se dá por uma combinação das ligas $\mathrm{AB}$ e $\mathrm{BC}$, nas proporções $\mathrm{A}_{x} \mathrm{~B}_{1-x} \mathrm{C}$, uma propriedade $P$ qualquer desta liga ternária será dada por:

$$
P\left(\mathrm{~A}_{x} \mathrm{~B}_{1-x} C\right)=x P(\mathrm{AB})+(1-x) P(\mathrm{BC})-x(1-x) c,
$$

onde $c$ é um parâmetro de ajuste chamado de bowing, geralmente determinado experimentalmente e que permite a introdução de não linearidades a interpolação simples. Este tipo de interpolação será utilizado para a obtenção de todos os parâmetros físicos da liga de InGaAs. Na Tabela 6 mostramos os valores de parâmetros do GaAs e do InAs, enquanto que na Tabela 7 mostramos os parâmetros de bowing do InGaAs, todos retirados da referência [48].

A dependência com a temperatura para o parâmetro $a_{0}$ é mostrada explicitamente na Tabela 6. Para o parâmetro $E_{\mathrm{g}}^{\Gamma}$ (gap), é utilizada a forma empírica de Varshni: [48]

$$
E_{\mathrm{g}}(T)=E_{\mathrm{g}}(T=0)-\frac{\alpha T^{2}}{T+\beta}
$$

A massa efetiva do buraco pesado $\left(m_{\mathrm{hh}}^{*}\right)$ é dada por: $[11,48]$

$$
m_{\mathrm{hh}}^{*}=\frac{1}{\gamma_{1}-2 \gamma_{2}}
$$

Tabela 6: Parâmetros do GaAs e do InAs. Todos os valores foram retirados da referência [48].

\begin{tabular}{lcc}
\hline Parâmetro & GaAs & InAs \\
\hline$a_{0}(\AA)$ & $5,65325+3,88 \times 10^{-5}(T-300)$ & $6,0583+2,74 \times 10^{-5}(T-300)$ \\
$E_{\mathrm{g}}^{\Gamma}(\mathrm{eV})$ & 1,519 & 0,417 \\
$\alpha(\Gamma)(\mathrm{meV} / \mathrm{K})$ & 0,5405 & 0,276 \\
$\beta(\Gamma)(\mathrm{K})$ & 204 & 93 \\
$m_{\mathrm{e}}^{*}(\Gamma)$ & 0,067 & 0,026 \\
$\gamma_{1}$ & 6,98 & 20,0 \\
$\gamma_{2}$ & 2,06 & 8,5 \\
$V B O(\mathrm{eV})$ & $-0,8$ & $-0,59$ \\
$a_{\mathrm{c}}(\mathrm{eV})$ & $-7,17$ & $-5,08$ \\
$a_{\mathrm{v}}(\mathrm{eV})$ & $-1,16$ & $-1,0$ \\
$b(\mathrm{eV})$ & $-2,0$ & $-1,8$ \\
$C_{11}(\mathrm{GPa})$ & 1221,0 & 832,9 \\
$C_{12}(\mathrm{GPa})$ & 566,0 & 452,6 \\
\hline
\end{tabular}


Tabela 7: Parâmetros de bowing do InGaAs. Todos os valores foram retirados da referência [48].

\begin{tabular}{lcc}
\hline Parâmetro & Unidade & InGaAs \\
\hline$E_{\mathrm{g}}^{\Gamma}$ & $\mathrm{eV}$ & 0,477 \\
$m_{\mathrm{e}}^{*}(\Gamma)$ & - & 0,091 \\
$V B O$ & $\mathrm{eV}$ & $-0,38$ \\
$a_{\mathrm{c}}$ & $\mathrm{eV}$ & 2,61 \\
\hline
\end{tabular}

\subsection{Segregação de In em Poços de InGaAs Crescidos Sobre Subs- tratos de $\mathrm{GaAs}$}

Um fenômeno bastante importante que ocorre em poços de InGaAs crescidos sobre GaAs é a chamada segregação dos átomos de In.[23, 24] Um cristal do tipo $\mathrm{In}_{x} \mathrm{Ga}_{1-x} \mathrm{As}$, por exemplo, $\mathrm{In}_{0,2} \mathrm{Ga}_{0,8}$ As deveria ter $50 \%$ dos pontos da rede ocupados por As, $20 \% \times 50 \%=10 \%$ dos pontos ocupados por In, e os restantes $80 \% \times 50 \%=40 \%$ ocupados por Ga.

Utilizando a técnica de MBE, esperava-se ser possível construir tal cristal controlando as frações dos elementos depositados sobre um substrato de GaAs. Entretanto, os elementos de In tendem a migrar para a superfície, deixando para trás uma monocamada de InGaAs com uma fração $x$ menor do que a pretendida $x_{0}$, também denominada concentração nominal. A fração $x_{0}$ acaba sendo atingida apenas após o crescimento de um determinado número de monocamadas.[23, 24, 34]

O fenômeno da segregação é esquematizado na Figura 26. Quando a primeira monocamada de $\operatorname{In}_{x_{0}} \mathrm{Ga}_{1-x_{0}}$ As é depositada sobre um substrato de GaAs, apenas uma porcentagem $(1-R)$ da fração pretendida $x_{0}$ acaba prevalecendo nesta monocamada, com o restante $(R)$ migrando para a superfície do material. Quando a segunda monocamada começa a ser preenchida, já existem átomos residuais de In na superfície (devidos à segregação ocorrida durante o crescimento da primeira monocamada), sendo agora a concentração de In igual a $x_{0}+x_{0} R$. Entretanto, também ocorrerá segregação nesta segunda monocamada, prevalecendo somente $\left(x_{0}+x_{0} R\right)(1-R)=x_{0}\left(1-R^{2}\right)$, com o restante $\left(x_{0}+x_{0} R\right) R=x_{0} R(1+R)$ migrando para a superfície. Este raciocínio, supondo que a segregação ocorrerá sempre desta forma contante, de acordo com um coeficiente $R$ fixo, foi sistematizado por Muraki e colaboradores (1992) [23] na expressão:

$$
x_{0}(n)= \begin{cases}x_{0}\left(1-R^{\mathrm{n}}\right) & \text { se } 1 \leq \mathrm{n} \leq \mathrm{N} \\ x_{0}\left(1-R^{\mathrm{N}}\right) R^{\mathrm{n}-\mathrm{N}} & \text { se } \mathrm{n}>\mathrm{N},\end{cases}
$$

onde $N$ é o número total de monocamadas de $\operatorname{In}_{x_{0}} \mathrm{Ga}_{1-x_{0}}$ As depositadas antes da barreira final de GaAs ser crescida sobre o poço. O coeficiente de segregação $R$ (como é formalmente 


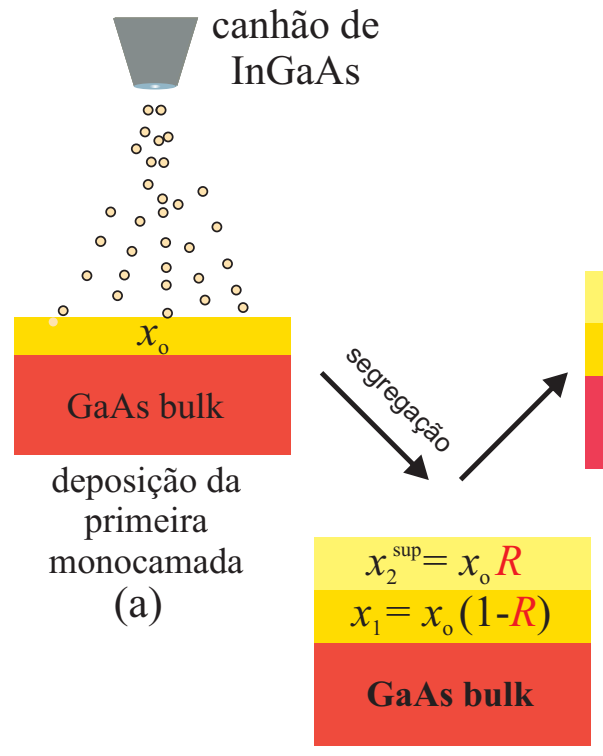

(b)

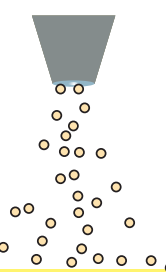

$x_{0} R+x_{0}$

$x_{\mathrm{o}}(1-R)$

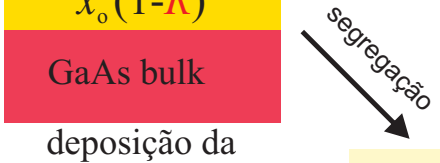

osição da segunda monocamada

(c)

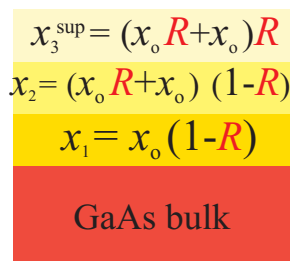

(d)

$$
\begin{array}{ll}
x_{\mathrm{n}}=\mathrm{x}_{\mathrm{o}}\left(1-R^{\mathrm{n}}\right) & \mathrm{n} \leq \mathrm{N} \\
x_{\mathrm{n}}=\mathrm{x}_{\mathrm{o}}\left(1-R^{\mathrm{n}}\right) R^{\mathrm{n}-\mathrm{N}} & \mathrm{n}>\mathrm{N}
\end{array}
$$

$x_{\mathrm{n}}=$ concentração de In na n-ésima monocamada

$x_{\mathrm{o}}=$ concentração nominal de In

$R=$ coeficiente de segregação que pode ser determinado por medidas de RHEED

$\mathrm{N}=$ número total de monocamandas de $\operatorname{In}_{x} \mathrm{Ga}_{1-x} \mathrm{As}$

Figura 26: Esquema explicativo do processo de segregação do In durante o crescimento epitaxial de $\mathrm{In}_{x_{0}} \mathrm{Ga}_{1-x_{0}}$ As sobre GaAs. São também indicadas as frações de In atingidas em cada monolayer segundo o modelo fenomenológico sugerido por Muraki e colaboradores (1992) [23], que pode ser conferido na Eq. (6.8).

referido) pode ser obtido experimentalmente durante o crescimento da estrutura através do RHEED (Reflection High-Energy Electron Diffraction).[46]

O resultado do efeito da segregação é um potencial como o da Figura 27. Dependendo do número de monocamadas depositadas, é possível que a fração de In $\left(x_{0}\right)$, inicialmente pretendida, nem chegue a ser atingida. É interessante observar que, como já contido na expressão (6.8), o In residual na superfície após o crescimento das monocamadas de InGaAs acaba sendo absorvido pelas monocamadas de GaAs puro depositadas na sequência. Dessa forma, uma barreira pura de GaAs só será atingida após a deposição de um determinado número de monocamadas.

Vale observar também que como cada monocamada de InGaAs terá uma fração $x$ de In diferente, não só o gap, mas todos os outros parâmetros (parâmetro de rede, massa efetiva, etc.) terão valores diferentes em cada monocamada. Sendo assim, o valor de $L_{1}$ na Figura 27 é igual ao número de monocamadas de GaAs multiplicado por metade do 


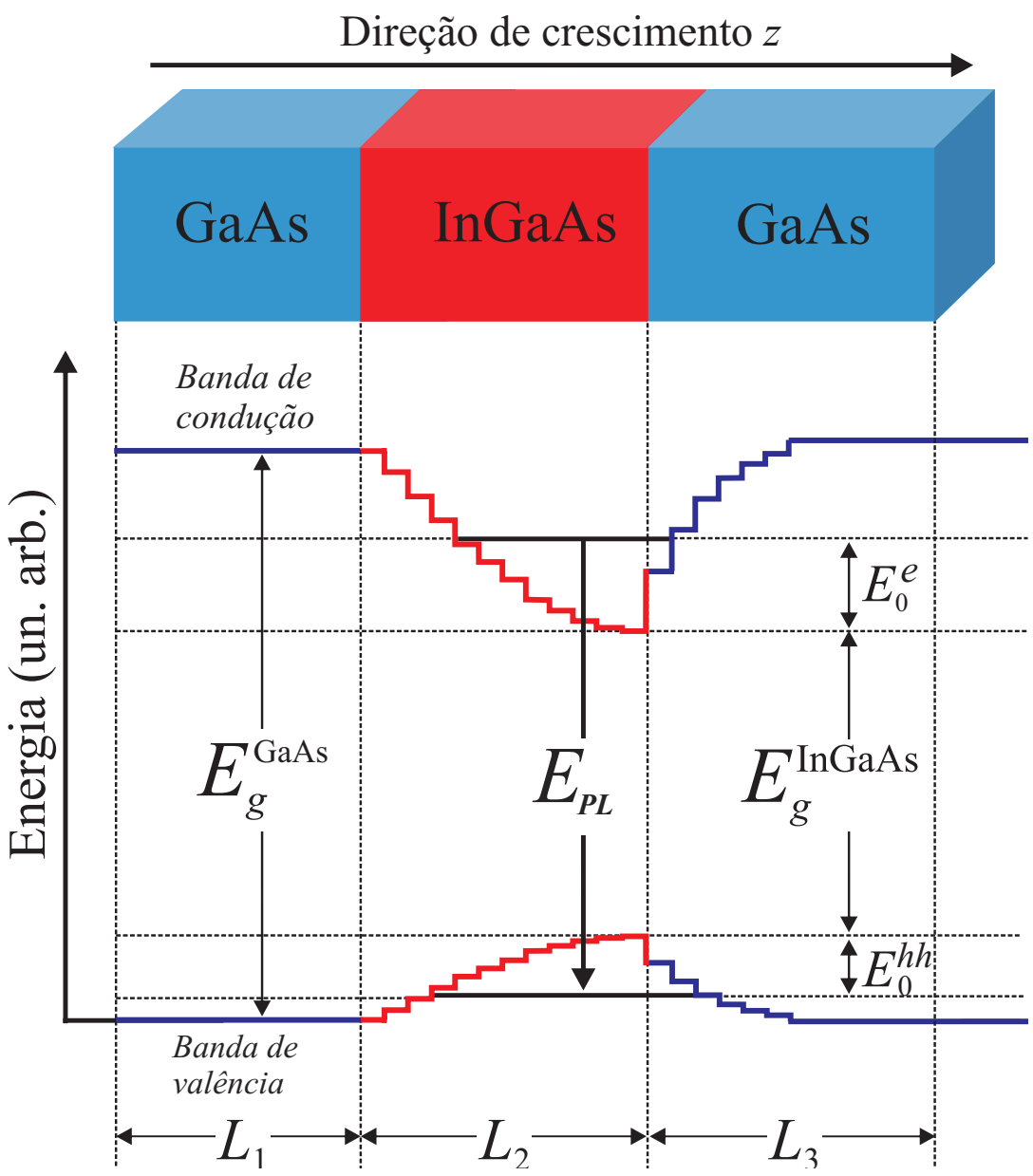

Figura 27: Perfil de potencial que ocorre em poços quânticos de InGaAs crescidos sobre GaAs devido ao efeito da segregação. Indicamos também as componentes da energia de um fóton emitido devido a recombinação de um elétron com um buraco pesado em uma experiência de PL, exceto pela energia de ligação do éxciton do material.

parâmetro de rede do GaAs, mas $L_{2}$ e $L_{3}$ são dados por

$$
\begin{aligned}
& L_{2}=\sum_{\mathrm{n}=1}^{\mathrm{N}} \frac{1}{2} a_{0}\left(x_{0}\left(1-R^{\mathrm{n}}\right)\right) \\
& L_{3}=\sum_{\mathrm{n}>\mathrm{N}} \frac{1}{2} a_{0}\left(x_{0}\left(1-R^{\mathrm{N}}\right) R^{\mathrm{n}-\mathrm{N}}\right),
\end{aligned}
$$

onde n é o número da monocamada começando a contar a partir da primeira monocamada de InGaAs, $\mathrm{N}$ é o total de monocamadas de InGaAs pretendidas e $a_{0}(x)$ são os parâmetros de rede indicados na Tabela 6.

\subsection{Cálculo das Energias de Transição de PL do Poço de InGaAs/ GaAs a partir de Modelagem Numérica}

Nesta seção, calculamos a energia do processo de recombinação do estado fundamental do elétron $\left(E_{0}^{e}\right)$ na banda de condução para o estado fundamental do buraco pesado 
$\left(E_{0}^{h h}\right)$ na banda de valência. Esta energia foi comparada com o resultado de medidas de fotoluminescência realizadas no LNMS.

A fotoluminescência é uma medida que consiste em excitar elétrons da banda de valência para a banda de condução, de modo que ao transitarem pela rede, estes elétrons percam energia até que atinjam o nível fundamental. Neste momento, eles se recombinam com buracos no nível fundamental da banda de valência. Os fótons emitidos através desta recombinação possuem um comprimento de onda bastante característico, representando a soma de quatro componentes [24]:

1. a energia do nível fundamental do elétron em relação ao fundo da banda de condução $\left(E_{0}^{e}\right)$

2. a energia do nível fundamental do buraco pesado em relação ao fundo da banda de valência $\left(E_{0}^{h h}\right)$;

3. o gap da liga de InGaAs que compõe a última camada do poço, levando em conta efeitos de tensão e segregação na temperatura em que a PL é realizada $\left(E_{\mathrm{g}}^{\mathrm{InGaAs}}\right)$;

4. a energia de ligação do éxciton do material, no caso o $\operatorname{In}_{x} \mathrm{Ga}_{1-x} \mathrm{As}$ (cuja fração $x$ é diferente em cada monocamada).

As componentes 1, 2 e 3 são exemplificadas na Figura 27, ao passo em que a energia de ligação do éxciton é tomada como sendo constante e igual a $7 \mathrm{meV}$.[24] Cálculos mais refinados revelam que esta energia varia entre $5 \mathrm{meV}$ e 7,5 meV.[34] Como nossos cálculos não incluem a interação coulombiana entre o elétron e o buraco no hamiltoniano, tomamos o valor constante como sendo uma boa primeira aproximação. Desta forma, a energia da PL é equivalente a:

$$
E_{\mathrm{PL}}=E_{\mathrm{g}}^{\mathrm{InGaAs}}+E_{0}^{e}+E_{0}^{h h}-0,007(\mathrm{eV}) .
$$

A amostra crescida no LNMS contém 4 poços quânticos de $\operatorname{In}_{0,14} \mathrm{Ga}_{0,86}$ As sobre GaAs, intercalados por barreiras de GaAs com larguras de aproximadamente $500 \AA$. O crescimento da amostra ocorreu a $525^{\circ} \mathrm{C}$ e o espectro de fotoluminescência (mostrado na Figura 28) foi medido a $77 \mathrm{~K}$. Os poços quânticos possuíam larguras de 10, 15, 21 e 28 monocamadas. Conforme detalhamos na Subseção 6.1, quando camadas de $\operatorname{In}_{x} \mathrm{Ga}_{1-x} \mathrm{As}$ são depositadas sobre GaAs, ocorre um tensionamento da rede cristalina, devido ao fato de o parâmetro de rede do $\operatorname{In}_{x} \mathrm{Ga}_{1-x}$ As ser consideravelmente maior que o do GaAs. Mostramos na Figura 29 os efeitos da tensão nas bandas de valência e de condução para o $\mathrm{In}_{0,14} \mathrm{Ga}_{0,86}$ As sobre GaAs a $77 \mathrm{~K}$. O valor do gap de $\operatorname{In}_{x} \mathrm{Ga}_{1-x}$ As não tensionado como função da temperatura foi calculado utilizando a expressão de Varshni, Eq. (6.6). As 


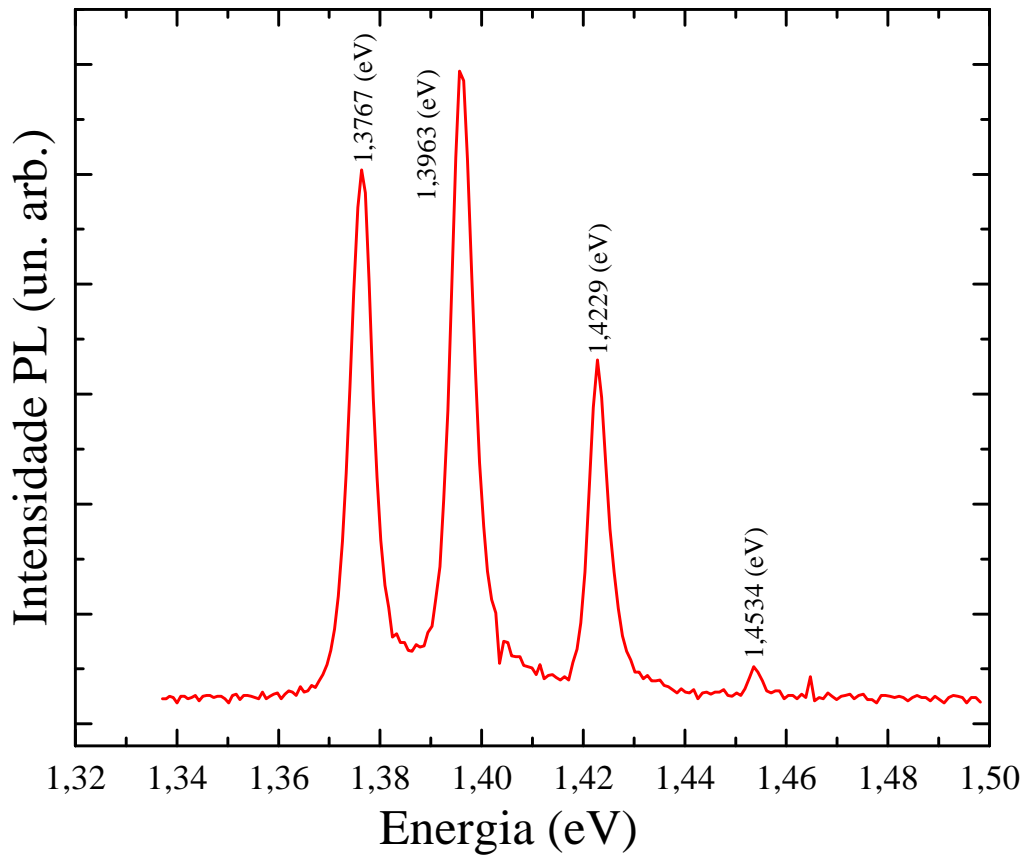

Figura 28: Espectro de PL de uma amostra crescida por MBE contendo 4 poços de $\operatorname{In}_{0,14} \mathrm{Ga}_{0,86} \mathrm{As} / \mathrm{GaAs}$.

correções devidas ao tensionamento para as bandas de valência e condução são dadas pelas expressões (6.2), (6.4) e (6.3).

Além dos efeitos de tensão, os poços quânticos de $\operatorname{In}_{x} \mathrm{Ga}_{1-x} \mathrm{As} / \mathrm{GaAs}$, diferentemente dos de $\mathrm{Al}_{x} \mathrm{Ga}_{1-x} \mathrm{As} / \mathrm{GaAs}$, não dão origem à perfis de potencial como os das Figuras

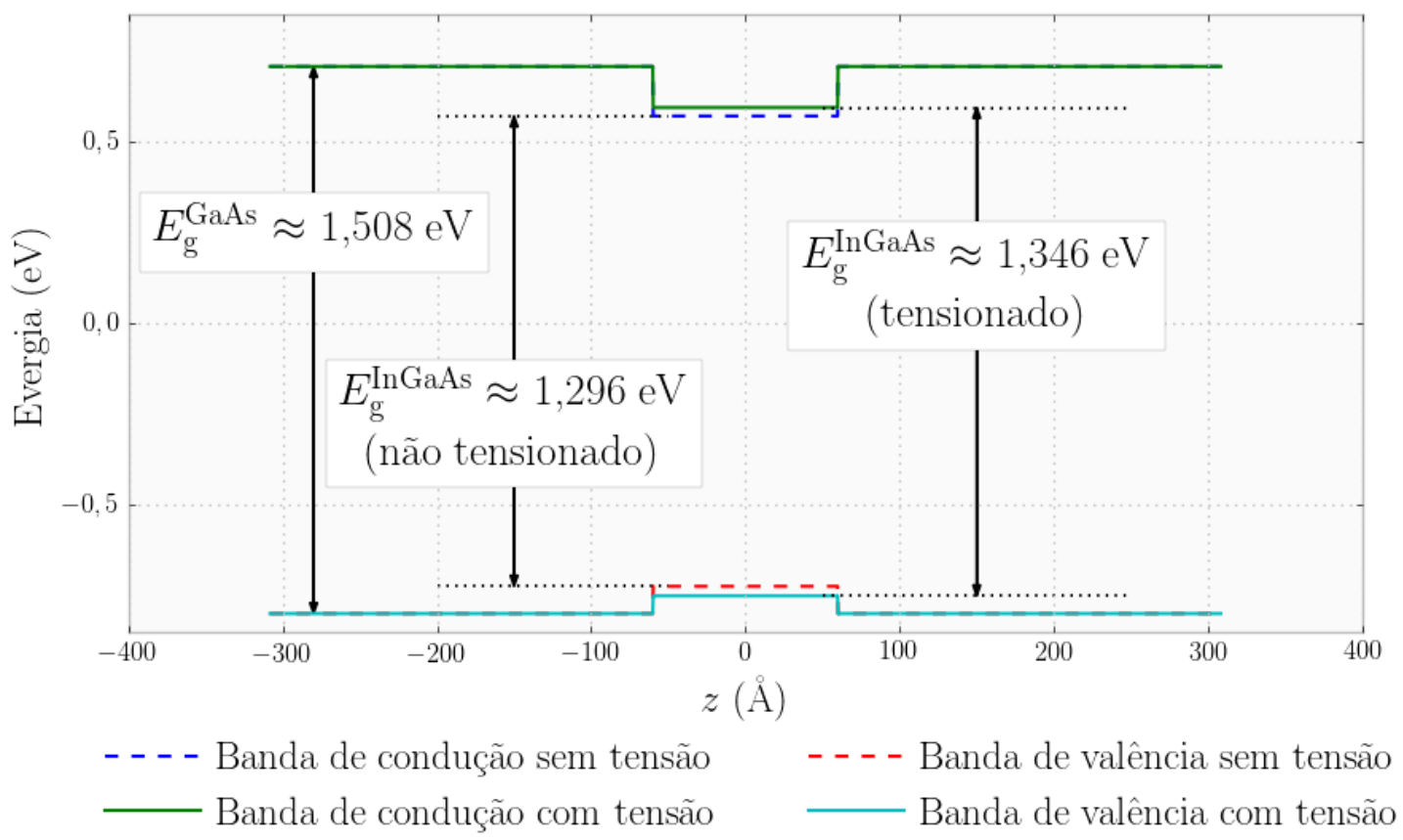

Figura 29: Efeito da tensão no gap do $\operatorname{In}_{0,14} \mathrm{Ga}_{0,86} \mathrm{AS}$ a $77 \mathrm{~K}$. As regiões a direita e a esquerda com gap $\Delta E_{g} \approx 1,508 \mathrm{eV}$ são constituídas de GaAs, sendo a região central constituída por $\operatorname{In}_{0,14} \mathrm{Ga}_{0,86}$ As. 


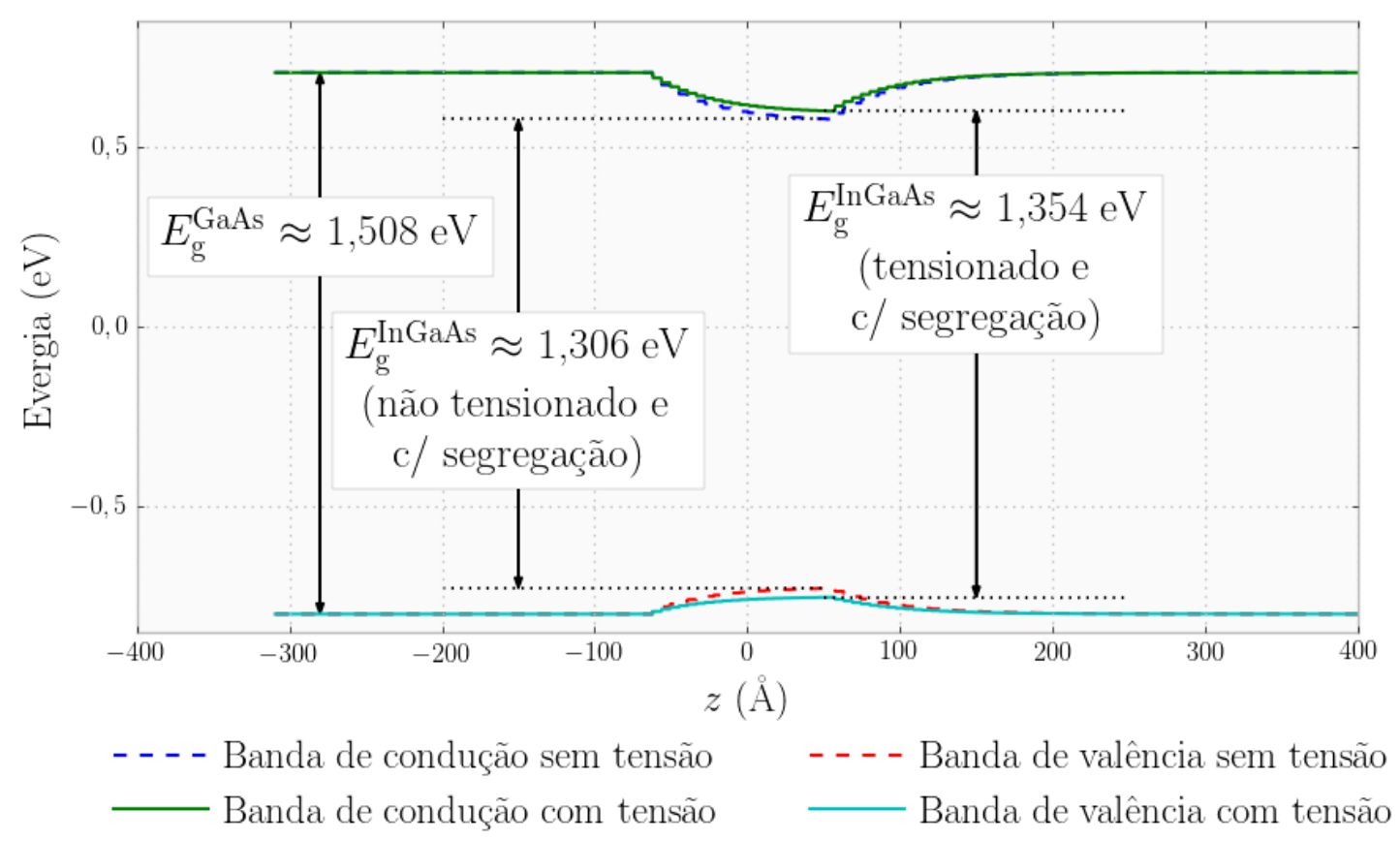

Figura 30: Perfil do potencial de um poço de 21 monocamadas de $\operatorname{In}_{0,14} \mathrm{Ga}_{0,86}$ As sobre GaAs a $77 \mathrm{~K}$ com correção de tensionamento e de segregação de acordo com modelo fenomenológico dado pela expressão (6.8) [23].

15, 17 e 29. O fenômeno da segregação, detalhado na Subseção 6.3, dificulta a fixação do In nos pontos na rede cristalina das primeiras camadas, sendo a fração pretendida inicialmente $x_{0}$ atingida apenas após um determinado número de camadas.[23, 24, 34] O resultado do efeito da segregação é um potencial como o da Figura 30. Dependendo do número de camadas depositadas, é possível que a fração de In $\left(x_{0}\right)$ inicialmente pretendida nem chegue a ser atingida.

Comparando as Figuras 29 e 30, vemos que, para 21 monocamadas, a fração de In $\left(x_{0}=0,14\right)$ inicialmente pretendida não chega a ser atingida. Isso pode ser inferido observando que o gap mínimo (tanto tensionado quanto não tensionado) é maior quando a segregação é considerada.

A fração real de In em cada camada é dada pela expressão (6.8). O valor do coeficiente de segregação utilizado foi $R=0,867$, obtido através de interpolação linear (para $T=525^{\circ} \mathrm{C}$ ) utilizando os valores da Tabela 8 .

Tabela 8: Coeficientes de segregação do Índio obtidos através de simulações de raios X para $\mathrm{In}_{0,15} \mathrm{Ga}_{0,85}$ As crescido sobre GaAs em diferentes temperaturas. Valores reportados pela referência [49].

\begin{tabular}{cc}
\hline Temperatura $\left({ }^{\circ} \mathrm{C}\right)$ & $\mathrm{R}$ \\
\hline 520 & 0,86 \\
460 & 0,75 \\
370 & 0,62 \\
\hline
\end{tabular}




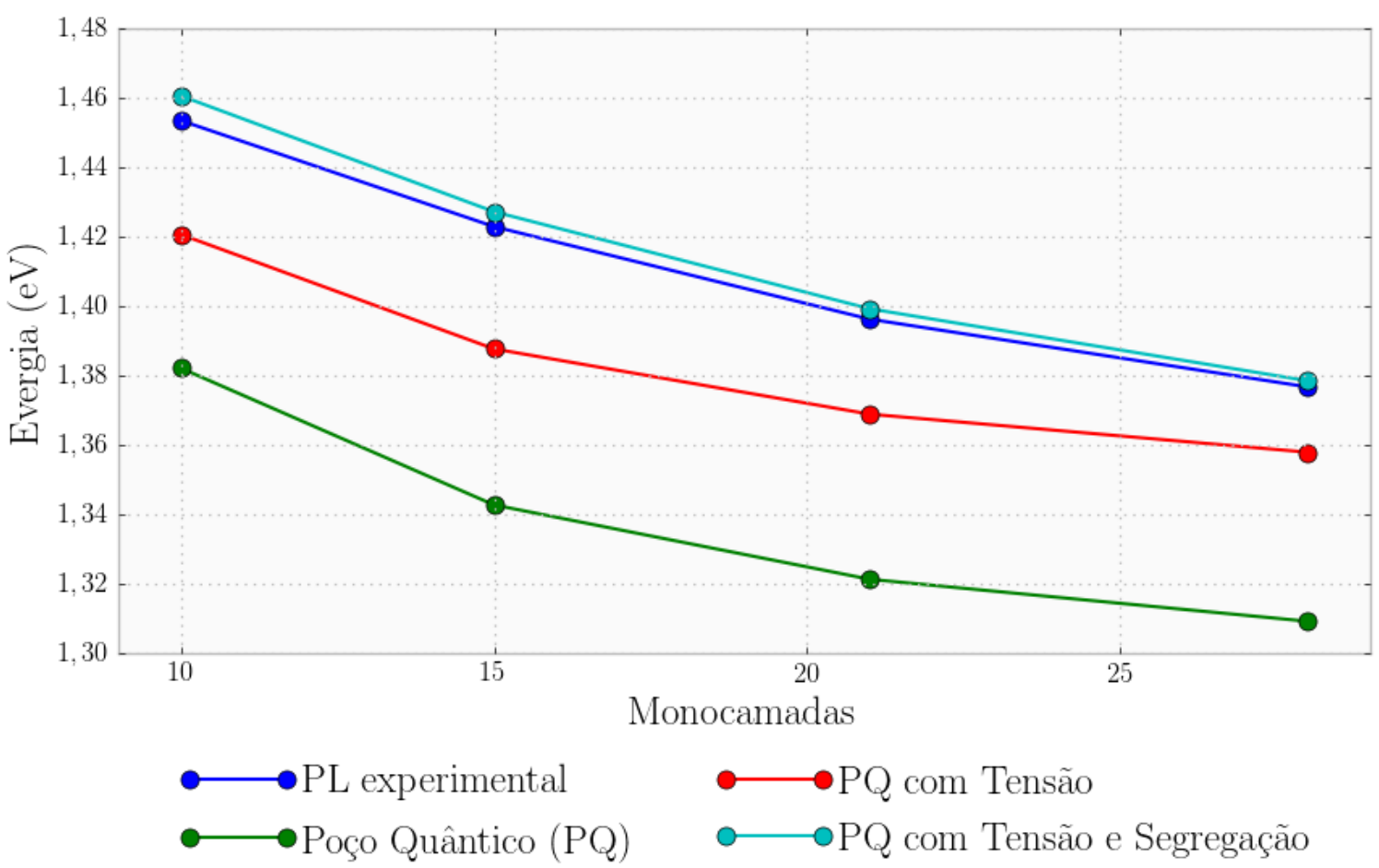

Figura 31: Comparação das energia de transição obtidos experimentalmente e numericamente para a $\mathrm{PL}$ (realizada a 77 )K dos quatro poços quânticos de larguras 10, 15, 21, 28 monocamadas, crescidos a $525^{\circ} \mathrm{C}$ no MBE do LNMS. A curva Poço Quântico (PQ) apresenta os níveis de energia em um poço quântico não tensionado de $\operatorname{In}_{0,14} \mathrm{Ga}_{0,86}$ As levando em conta somente o gap dado pela expressão (6.6). A curva PQ com Tensão acrescenta o fenômeno da tensão. Finalmente, a curva PQ com Tensão e Segregação considera os dois efeitos no cálculo dos níveis de energia. Apesar de PQ indicar poço quântico, o fato de a segregação modificar totalmente a estrutura, esta nomenclatura acaba sendo um abuso de linguagem.

Nós utilizamos a Eq. (6.10) para calcular as energias de recombinação de cada um dos quatro poços presentes na amostra. Para evidenciar os efeitos da tensão e da segregação, efetuamos inicialmente uma simulação para um poço quântico não tensionado, na sequência adicionando a tensão e por fim a segregação, como é possível ver na Figura 31. O algoritmo utilizado para o cálculo dos níveis de recombinação levando em conta os efeitos de tensão e segregação pode ser verificado no Anexo A.6. 



\section{Conclusão}

Neste trabalho desenvolvemos procedimentos numéricos para resolver a Equação de Schrödinger dependente do tempo (ESDT) com o intuito de calcular propriedades dinâmicas (espectros de fotocorrente e absorção) de heteroestruturas semicondutoras que são utilizadas para o desenvolvimento de dispositivos do Estado Sólido.

Um dos métodos usados neste trabalho é o Pseudo-Espectral, tanto para encontrar autovalores e autovetores (através da técnica de propagação em tempo imaginário) quanto para evoluir o estado inicial no tempo, o que é equivalente a resolver a ESDT. Nossas investigações mostram que apesar de o Método Pseudo-Espectral ser bastante versátil, ele possui algumas limitações. No problema da evolução temporal, ele não pode ser utilizado para sistemas onde a variação espacial da massa efetiva do elétron é importante, como é no caso de heteroestruturas semicondutoras onde os portadores nos diferentes materiais têm diferentes massas efetivas. Já no problema de encontrar autovalores e autovetores, ele é significativamente mais lento do que o método da Iteração Inversa, que também foi utilizado no desenvolvimento desta dissertação. Este último por sua vez é extremamente eficaz, mas depende do conhecimento prévio (mesmo que aproximado) dos autovalores.

Para diferenciar e resumir os dois problemas que buscamos resolver através de métodos numéricos criamos o diagrama mostrado na Figura 32. No caso de evolução temporal não existem muitas alternativas. Caso o sistema analisado possua uma importante dependência na variação espacial da massa efetiva do elétron, devem ser usados os métodos de Runge-Kutta ou Crank-Nicolson; caso a massa efetiva do elétron possa ser considerada constante ao longo de todo o sistema, deve-se então optar pelo método Pseudo-Espectral, que é substancialmente mais rápido e preciso. Entre os métodos de Runge-Kutta e CrankNicolson, o último tende a ser mais preciso na conservação da norma da função de onda, mas se torna muito custoso quando o sistema possui um hamiltoniano com dependência temporal, enquanto que o primeiro é um meio termo das duas situações: não é tão preciso, mas também não se torna tão ineficiente quando o sistema possui um hamiltoniano com dependência temporal.

No caso do problema de autovalores e autovetores, algumas abordagens distintas podem ser adotadas. Se os autovalores forem conhecidos, mesmo que aproximadamente, deve-se sempre optar pelo método da Iteração Inversa, pois ele é extremamente rápido, permite levar em conta a variação espacial da massa efetiva do elétron e funciona muito bem com malhas espaciais com espaçamentos não uniformes ${ }^{1}$. No caso de os autovalores

1 Este procedimento permite que mais pontos da malha espacial sejam dedicados à região ativa e menos pontos às bordas. Entretanto, não identificamos melhora significativa de precisão adotando este procedimento, ao passo que houve grande diminuição da performance. 


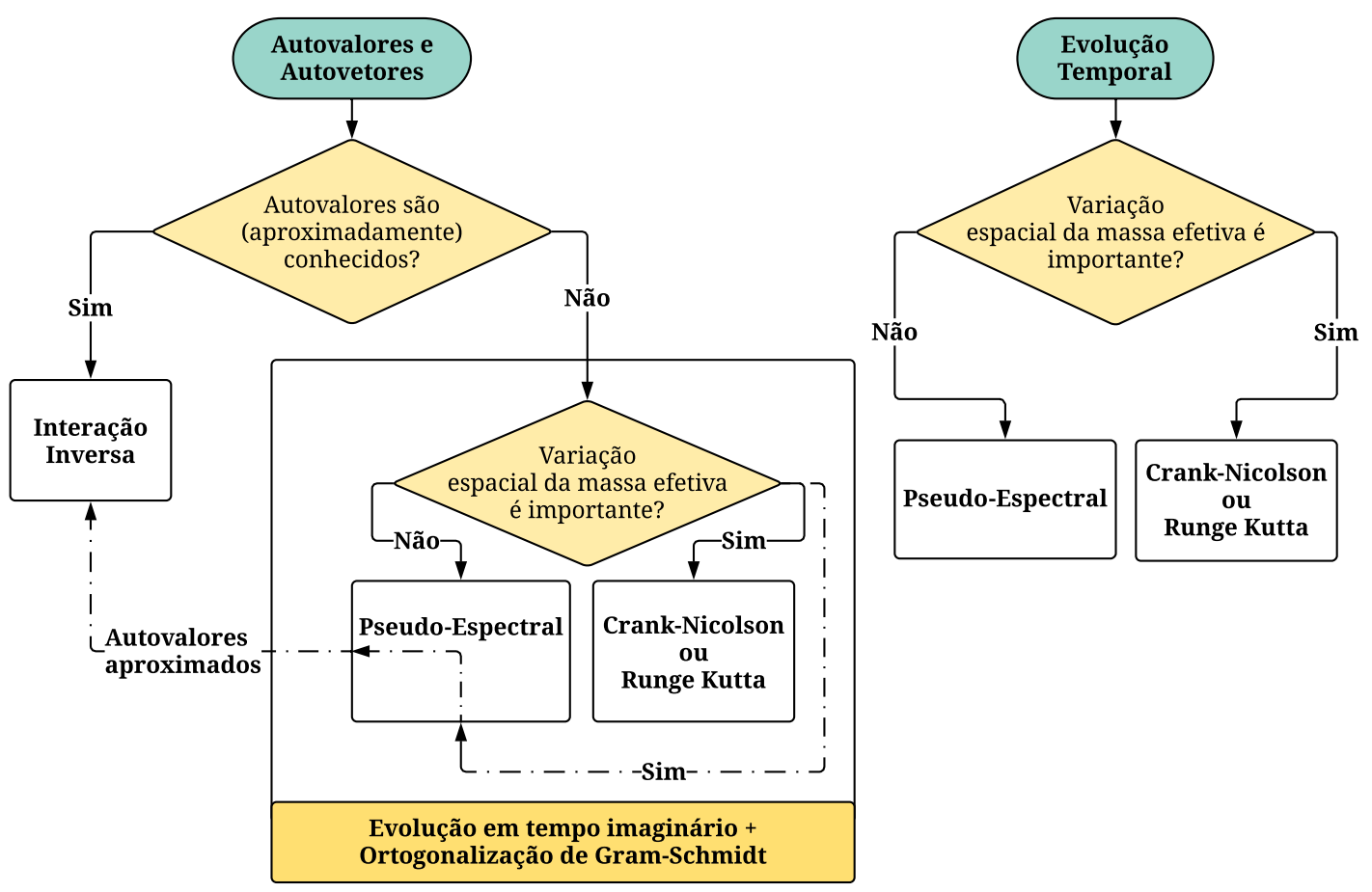

Figura 32: Diagrama para escolha do método numérico a ser utilizado dependendo do problema que se deseja resolver.

não serem conhecidos, este método ainda pode ser adotado, desde que um intervalo de valores seja utilizado como valores iniciais. Esta última abordagem é bastante ruim porque muitos chutes iniciais convergem para o mesmo autoestado e muitas vezes alguns autoestados são perdidos, ou seja, não são encontrados. A única forma de garantir a obtenção de todos os autoestados utilizando esta abordagem é utilizando um intervalo com muitos valores, como por exemplo 10 mil pontos no intervalo $[-1 \mathrm{eV},+1 \mathrm{eV}]$, o que faz com que o método acabe levando mais tempo do que o método Pseudo-Espectral, que não falha em identificar corretamente todos os autoestados.

Caso os autovalores não sejam conhecidos de início, sugere-se utilizar a evolução em tempo imaginário para obtenção do estado fundamental juntamente com a ortogonalização de Gram-Schmidt para obtenção dos estados excitados. Esta evolução em tempo imaginário pode ser feita tanto pelo método Pseudo-Espectral quanto pelos métodos de Runge-Kutta ou Crank-Nicolson. A escolha de qual deles utilizar depende da importância que a variação espacial da massa efetiva do elétron tem no sistema. Se é possível considerá-la constante ao longo de todo o sistema, deve-se então optar pelo método Pseudo-Espectral, caso contrário deve-se optar por um dos outros dois. A melhor estratégia no caso de a variação espacial da massa efetiva do elétron precisar ser considerada é utilizar o método Pseudo-Espectral para obter uma aproximação para os autovalores e utilizá-los como chutes iniciais para o método da Iteração Inversa.

O desenvolvimento de um ferramental confiável para lidar com estes dois tipos de 
problema nos permitiu calcular níveis de energia e algumas propriedades dinâmicas de heteroestruturas semicondutoras como a fotocorrente e o espectro de absorção. Optamos por aprimorar as técnicas exaustivamente ao resolver problemas com soluções analíticas ou reportadas na literatura, para ganhar confiança e avançar para o cálculo dos níveis de uma amostra produzida pelo MBE do LNMS do IFUSP.

O domínio das técnicas computacionais, entretanto, é metade do trabalho. A outra metade diz respeito a compreensão de como a Mecânica Quântica fundamenta a natureza na escala trabalhada, bem como um verdadeiro malabarismo realizado na tentativa de preencher as lacunas com modelos semi-clássicos e até mesmo fenomenológicos. Para bem da verdade, esta última parte acaba sendo a parte mais gratificante, pois é quando a maioria dos conceitos físicos começam de fato a serem questionados.

A teoria que fundamenta a obtenção e evolução temporal dos autoestados é baseada puramente na Mecânica Quântica, mas as aproximações que são feitas acerca da redução de dimensionalidade e sobre a massa efetiva do elétron vêm da teoria do Estado Sólido. A interação da radiação com a matéria, utilizada no cálculo do espectro de absorção é baseada em uma teoria semi-clássica, onde a hamiltoniana do sistema é modificada para acomodar campos eletromagnéticos clássicos, que são ótimas aproximações nas escalas de tempo do sistema. Merece destaque também a expressão fenomenológica de Muraki et al [23] utilizada para modelar o fenômeno de segregação do In em poços quânticos de InGaAs/GaAs.

A compreensão dos métodos numéricos bem como sua correta utilização na modelagem de sistemas constituídos por heteroestruturas semicondutoras nos abre um novo caminho pela frente. Certamente que ainda precisamos aprimorar algumas técnicas relacionadas ao cálculo de propriedades dinâmicas, mas já obtivemos alguns resultados positivos no cálculo de propriedades de sistemas mais complexos como detetores e lasers de cascata quântica, que são os dispositivos que objetivamos estudar e modelar nas próximas etapas. 



\section{Referências}

1 Solution of the Schrödinger equation by a spectral method, M.D. Feit, J.A. Fleck and A. Steiger, J. of Computational Phys. 47, 412-433 (1982). Citado 10 vezes nas páginas $13,14,39,46,47,48,55,68,69$ e 115 .

2 Solution of the Schrödinger equation by a spectral method II: Vibrational energy levels of triatomic molecules, M.D. Feit and J.A. Fleck, J. Chem. Phys. 78(1), 301-308 (1983). Citado 4 vezes nas páginas 13, 46, 47 e 48.

3 A Fourier method solution for the time dependent Schrödinger equation as a tool in molecular dynamics, D. Kosloff and R. Kosloff, J. Comp. Phys. 52(1), 35-53 (1983). Citado 3 vezes nas páginas 13, 48 e 72.

4 An accurate and efficient scheme for propagating the time dependent Schrödinger equation, H. Tal-Ezer and R. Kosloff, J. Chem. Phys. 81(9), 3967-3971 (1984). Citado na página 13.

5 Unitary quantum time evolution by iterative Lanczos reduction, T.J. Park and J.C. Light, J. Chem. Phys. 85(10), 5870-5876 (1986). Citado na página 13.

6 Infrared photocurrent with one- and two-photon absorptions in a double-barrier quantum well system, M.H. Degani, M.Z. Maialle, P.F. Farinas and N. Studart, J. Appl. Phys. 110, 104313 (2011). Citado 15 vezes nas páginas 14, 20, 21, 22, 26, 39, 53, 55, 70, $71,72,73,74,75$ e 122 .

7 V.V. Mitin, V.A. Kochelap and M.A. Stroscio em Quantum Heterostructures: Microelectronics and Optoelectronics, editado por Cambridge University Press (1999). ISBN: 0521636353. Citado 6 vezes nas páginas 15, 36, 37, 64, 72 e 80.

8 J.J. Sakurai em Modern Quantum Mechanics, editado por Addison Wesley (1994). ISBN: 0-201-53929-2. Citado 3 vezes nas páginas 16, 22 e 25.

9 D.J. Tannor em Introduction to Quantum Mechanics - A Time-Dependent Perspective, editado por University Science Books (2007). ISBN: 1-891389-23-8. Citado 5 vezes nas páginas 17, 25, 26, 27 e 39.

10 Max Born em On the Quantum Mechanics of Collisions, editado por Princeton University Press (1983), 863-867. ISBN: 0-691-08316-9. Citado na página 18.

11 S.L. Chuang em Physics of Optoelectronic Devices, editado por John Wiley \& Sons (1995). ISBN: 0-471-10939-8. Citado 11 vezes nas páginas 20, 22, 27, 31, 36, 37, 38, 64, 78,79 e 80. 
12 D.A.B. Miller em Quantum Mechanics for Scientists and Engineers, editado por Cambridge University Press (2010). ISBN: 978-0-521-89783-9. Citado 7 vezes nas páginas 22, 26, 31, 34, 35, 36 e 38.

13 G. Bastard em Wave Mechanics Applied to Semiconductor Heterostructures, editado por Les Édition de Physique (1988). ISBN: 0-470-21708-1. Citado na página 24.

14 Real-time time-dependent electronic structure theory, J.J. Goings, P.J. Lestrange, X. Li, Wiley Interdisciplinary Reviews: Computational Molecular Science, 8(1), e1341 (2017). Citado 2 vezes nas páginas 26 e 27.

15 Superlattice and negative differential conductivity in semiconductors, L. Esaki and R. Tsu, IBM Journal of Research and Development 14(1), 61-65 (1970). Citado na página 31.

16 Resonant tunneling in semiconductor double barriers, L.L. Chang, L. Esaki and R. Tsu, Appl. Phys. Lett. 24(12), 593-595 (1974). Citado na página 31.

17 Molecular beam epitaxy, J.R. Arthur, Surface Science 500(1), 189-217 (2002). Citado 2 vezes nas páginas 31 e 36.

18 Progress in chemical beam epitaxy, W.T. Tsang, Journal of Crystal Growth 105(1), 1-29 (1990). Citado na página 31.

19 MOCVD technology for semiconductors, A.G. Thompson, Materials Letters 30(4), 255-263 (1997). Citado na página 31.

20 Electron-electron and electron-hole interactions in small semiconductor crystallites:

The size dependence of the lowest excited electronic state, L.E. Brus, J. Chem. Phys. 80(9), 4403-4409 (1984). Citado na página 31.

21 Strained-layer superlattices from lattice mismatched materials, G.C. Osbourn, J. Appl. Phys. 53(3), 1586-1589 (1982). Citado na página 31.

22 Frequency doubling of an InGaAs multiple quantum wells semiconductor disk laser, J. Lidan, Z. Renjiang, J. Maohua, Z. Dingke, C. Yuting, Z. Peng and S. Yanrong, Superlattices and Microstructures 113, 785-790 (2018). Citado na página 31.

23 Surface segregation of In atoms during molecular beam epitaxy and its influence on the energy levels in InGaAs/GaAs quantum wells, K. Muraki, S. Fukatsu, Y. Shiraki and R. Ito, Appl. Phys. Lett. 61, 557 (1992). Citado 5 vezes nas páginas 32, 81, 82, 86 e 91.

24 Study of the influence of indium segregation on the optical properties of InGaAs/GaAs quantum wells via split-operator method, S. Martini, J.E. Manzoli and A.A. Quivy, J. Vacuum Science \& Technology B 28(2), 277-283 (2010). Citado 7 vezes nas páginas 32, $39,53,78,81,84$ e 86 . 
25 Energy-band structure of GaAs and Si: $A \mathrm{sps}^{*} k \cdot p$ method, N. Cavassilas, F. Aniel, K. Boujdaria and G. Fishman, Phys. Rev. B 64(11), 115207 (2001). Citado na página 32.

26 C. Kittel em Introdução à Fúsica do Estado Sólido, editado por LTC (2006). ISBN: 85-216-1505-1. Citado 2 vezes nas páginas 33 e 34.

27 Eigenvalue problem of the Schrödinger equation via the finite-difference time-domain method, G.B. Ren and J.M. Rorison, Phys. Rev. E 69(3), 036705 (2004). Citado na página 39.

28 Determining quantum eigenfunctions in three-dimensional nanoscale structures, D.M. Sullivan and D.S. Citrin, J. Appl. Phys. 97(10), 104305 (2005). Citado na página 39.

29 Modeling of strained quantum wires using eight-band $k \cdot p$ theory, O. Stier and D. Bimberg, Phys. Rev. B 55, 7726-7732 (1997). Citado na página 39.

30 Lanczos-type algorithm for excited states of very-large-scale quantum systems, $\mathrm{G}$. Grosso, L. Martinelli and G.P. Parravicini, Phys. Rev. B 51(19), 13033-13038 (1995). Citado na página 39.

31 Theory of line shapes of exciton resonances in semiconductor superlattices, $\mathrm{H}$. Chu and Y.C. Chang, Phys. Rev. B 39(15), 10861-10871 (1989). Citado na página 39.

32 Exact numerical solution of the Schörodinger equation for a particle in an interaction potential of general shape, M. Rieth, W. Schommers and S. Baskoutas, Int. J. Modern Phys. B 16(27), 4081-4092 (2002). Citado na página 39.

33 Numerical calculations of quantum states in semiconductor nanostructures, M.H. Degani and M.Z. Maialle, J. of Computational and Theoretical Nanoscience 7(27), 454-473 (2010). Citado na página 39.

34 Theoretical study of the indium incorporation into III-V compounds revisited: The role of indium segregation and desorption, R.R Pelá, L.K. Teles, M. Marques and S. Martini, J. Appl. Phys. 113, 033515 (2013). Citado 7 vezes nas páginas 39, 54, 78, 79, 81,84 e 86.

35 D. Sperandio, J.T. Mendes and L.H.M. Silva em Cálculo Numérico, editado por Pearson (2003). ISBN: 85-87918-74-5. Citado 6 vezes nas páginas 39, 42, 43, 44, 45 e 54.

36 P.L. Devries em A First Course in Computational Physics, editado por John Wiley and Sons (1994). ISBN: 0-471-54869-3. Citado 3 vezes nas páginas 46, 50 e 56.

37 Wave packet dynamics and chaos in the henon-heiles system, M.D. Feit and J.A. Fleck, J. Chem. Phys. 80(6), 2578-258 (1984). Citado 2 vezes nas páginas 46 e 47. 
38 An algorithm for the machine calculation of complex Fourier series, J.W. Cooley and J.W. Tukey, Math. Comput. 19, 297-301 (1965). Citado na página 51.

39 Historical notes on the fast Fourier transform, J.W. Cooley, P.A.W. Lewis and P.D. Welch, Proceedings of the IEEE 55(10), 1675-1677 (1967). Citado na página 51.

40 J.L. Powell and B. Crasemann em Quantum Mechanics, editado por Addison-Wesley Pub. Co. (1961). Citado 2 vezes nas páginas 56 e 61.

41 M.J. Oliveira and T. Tomé em Dinâmica Estocástica e Irreversibilidade, editado pela EDUSP (2014). ISBN: 9788531414800. Citado 2 vezes nas páginas 57 e 58.

42 Using the OWA operator in the Minkowski distance, J.M. Merigó and A. M. Gil-Lafuente, Int. J. Computer Science 3(3), 149-157 (2008). Citado na página 58.

43 Evaluation of transition probabilities in a double asymmetric potential well, N.I. Zhirnov and A.V. Turev, Optics and Spectroscopy 47, 480-484 (1979). Citado 3 vezes nas páginas 68, 69 e 115.

44 Parametrization of complex absorbing potentials for time-dependent quantum dynamics, A. Vibok and G.G. Balint-Kurti, J. Physical Chemistry 96(22), 8712-8719 (1992). Citado na página 72.

45 Band lineups and deformation potentials in the model-solid theory, C.G. Van de Walle, Phys. Rev. B 39(3), 1871-1883 (1989). Nenhuma citação no texto.

46 Influence of indium segregation on the RHEED oscillations during the growth of InGaAs layers on a GaAs(001) surface, S. Martini, A.A. Quivy, T.E. Lamas, M.J. da Silva, E.C.F. da Silva and J.R. Leite, J. of Crystal Growth 251(1), 101-105 (2003). Citado na página 82.

47 Temperature dependence of the photoluminescence of $\operatorname{In}_{x} G a_{1-x} A s / G a A s$ quantumwell structures, M. V. Karatchevtseva, A.S. Ignatiev, V.G. Mokerov, G.S. Nemtsovi, V.A. Strakhov, and N.G. Yaremenko, Semiconductors 28(7), 691-694 (1994). Nenhuma citação no texto.

48 Band parameters for III-V compound semiconductors and their alloys, I. Vurgaftman and J. R. Meyer, Journal of Applied Physics 89(11), 5815-5875 (2001). Citado 5 vezes nas páginas 37, 78, 79, 80 e 81 .

49 Estudo da Segregação de Índio em Camadas Epitaxiais de $\operatorname{In}_{x} G a_{1-x}$ As Crescidas sobre Substratos de GaAs(001), S. Martini. Tese (Doutorado em Física) - Instituto de Física, Universidade de São Paulo. São Paulo, p. 122. 2002. Citado na página 86. 


\section{ANEXO A - Algoritmos}

Neste anexo documentamos todos os algoritmos utilizados na obtenção dos gráficos e resultados numéricos que são apresentados ao longo de toda a dissertação. Todos estes códigos também podem ser acessados no endereço https://github.com/thiagolcmelo/ mestrado.

\section{A.1 Evolução Temporal: Pacote de Onda Plana}

O algoritmo à seguir implementa a solução analítica para a evolução temporal de um pacote de onda utilizando um método numérico de integração (Regra de Simpson) além de transformadas diretas e inversas de Fourier, também numéricas.

Como a solução analítica para o problema ficou dependente apenas de quadraturas, um problema para o qual entendemos existirem métodos numéricos bastante eficazes, optamos por chamar essa solução de pseudo-analítica.

Além da solução pseudo-analítica, este algoritmo permite a evolução numérica através de três métodos: Pseudo-Espectral, Crank-Nicolson e Runge-Kutta.

Como forma de demonstração, as soluções numéricas para todos os métodos, tamanhos diferentes de sistemas, diferentes números de pontos e de passos de tempo, são comparadas com as respectivas soluções analíticas.

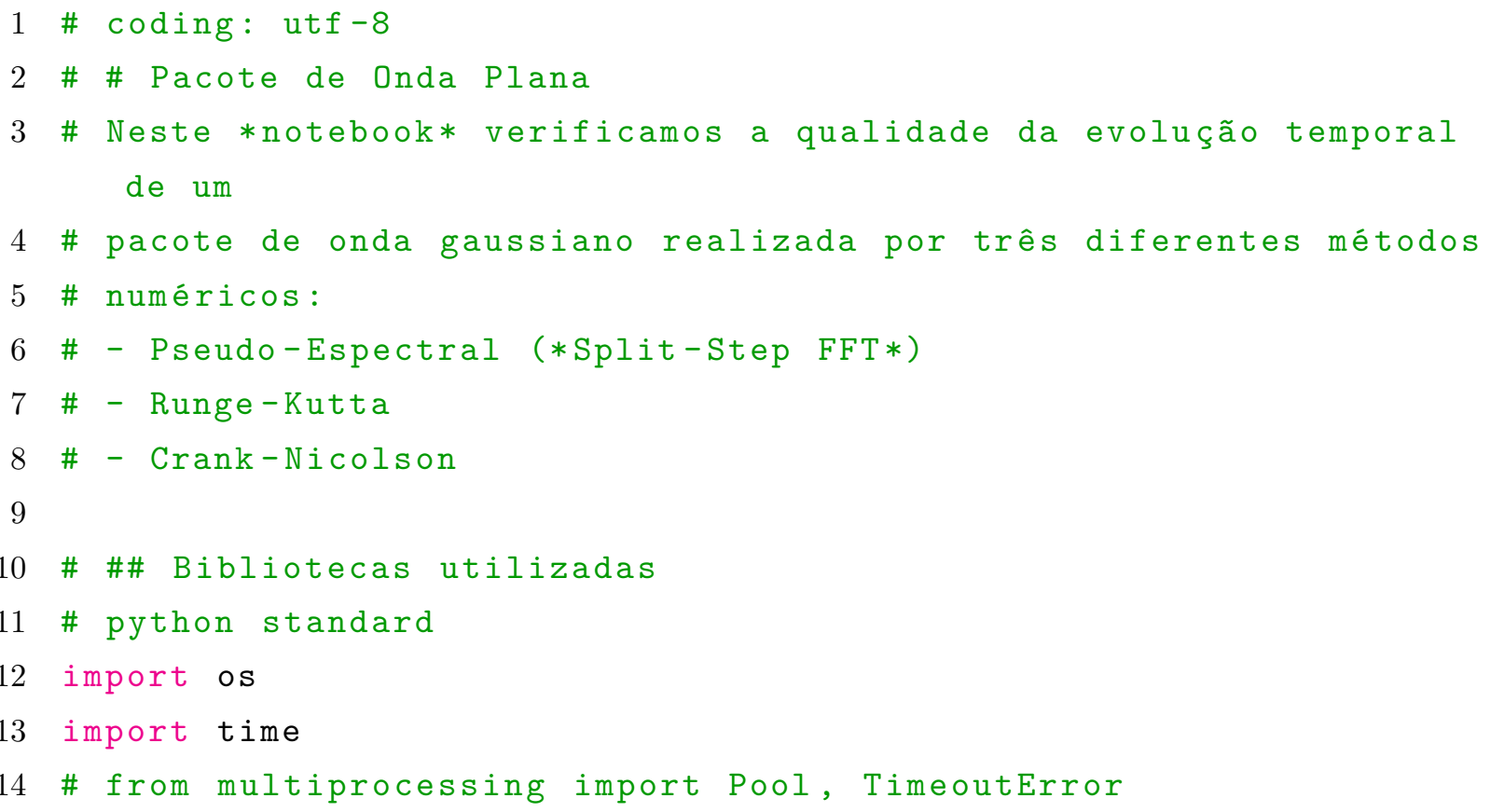


15 \# third-party

16 import numpy as np

17 import pandas as pd

18 from scipy.stats import norm, skewnorm

19 from scipy.spatial.distance import cdist

20 from sklearn.preprocessing import StandardScaler

21 \# locals

22 from core.constantes import *

23 from core.utilidades import *

24 from core.onda_plana import evolucao_analitica, evolucao_numerica

26 \# \#\# Configurações gráficas

27 import locale

28 locale.setlocale (locale.LC_NUMERIC, "pt_BR.UTF-8")

29 import matplotlib.style

30 import matplotlib as $\mathrm{mpl}$

31 mpl.style.use ('classic')

32 get_ipython().run_line_magic('matplotlib', 'inline')

33 import matplotlib.pyplot as plt

34 plt.style.use ('mestrado')

35

$36 \%$ \#\# Parâmetros de qualidade

37 \% Vamos exemplificar abaixo dois dos parâmetros de qualidade:

$38 \%$ \# - dispersão ou devio padrão

$39 \%$ - obliquidade ou *skewness*

$40 \% \mathrm{x}=\mathrm{np}$. Iinspace $(-10,10,1000)$

$41 \%$ df $=$ pd. DataFrame $(\{$

$42 \% \quad$ ' $\mathrm{x}$ ': $\mathrm{x}$,

$43 \% \quad$ 'low_standard_deviation': norm.pdf (x, loc =0, scale=0.5),

$44 \%$ 'normal_standard_deviation': norm.pdf (x, loc=0, scale=2.0),

$45 \% \quad$ 'high_standard_deviation': norm.pdf (x, loc=0, scale=4.0),

$46 \% \quad$ 'negative_skewness': skewnorm.pdf (x, $-2,10 c=0$, scale $=2.0)$,

$47 \% \quad$ 'zero_snewness': skewnorm.pdf (x, 0, loc=0, scale=2.0),

$48 \% \quad$ 'positive_skewness': skewnorm.pdf (x, 2, loc=0, scale=2.0)

$49 \% \quad\})$

$50 \% \mathrm{f},((\operatorname{ax} 1, \operatorname{ax} 2, \operatorname{ax} 3),(\operatorname{ax} 4, \operatorname{ax} 5, \operatorname{ax} 6))=\operatorname{plt} . \operatorname{subplots}(2,3$, sharey= True )

$51 \%$ mean, var, skew, kurt = norm.stats (moments='mvsk')

$52 \%$ ax1.plot ( $x, \operatorname{norm} \cdot \operatorname{pdf}(\mathrm{x}, \mathrm{loc}=0, \mathrm{scale}=0.5),{ }^{\prime} \mathrm{r}-{ }^{\prime}, \mathrm{alpha}=0.6$,

$53 \% \quad$ label='norm pdf')

$54 \% \operatorname{ax} 1 . \operatorname{text}(-8,0.6$, r"\$ $\$$ sigma $<\backslash$ sigma_0\$")

$55 \%$ ax2.plot $\left(x, \operatorname{norm} \cdot \operatorname{pdf}(\mathrm{x}, \mathrm{loc}=0, \mathrm{scale}=2.0),{ }^{\prime}{ }^{-}\right.$', , $\mathrm{lpha}=0.6$, 


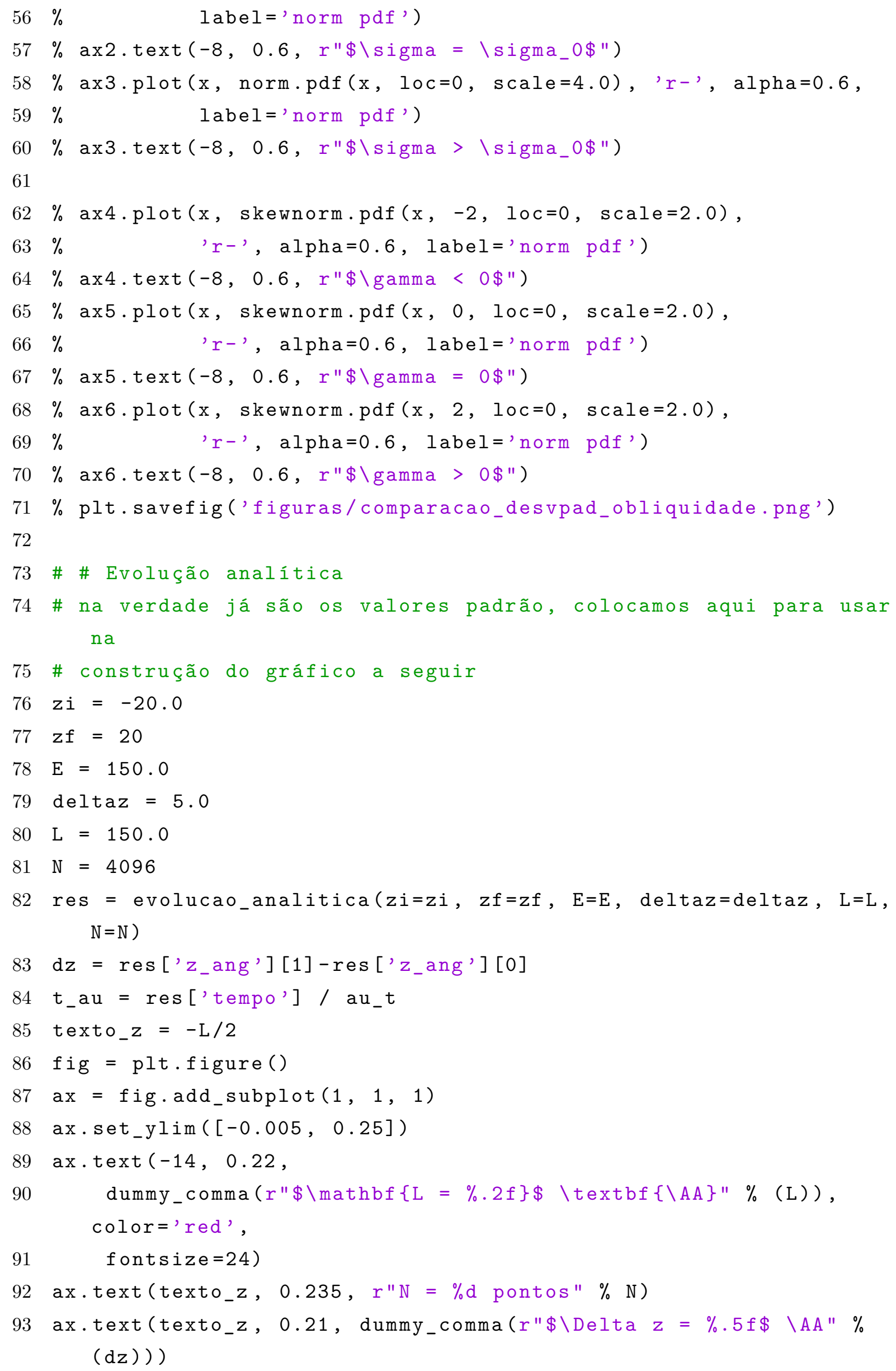


115

116

ax.text (texto_z, 0.18, dummy_comma (

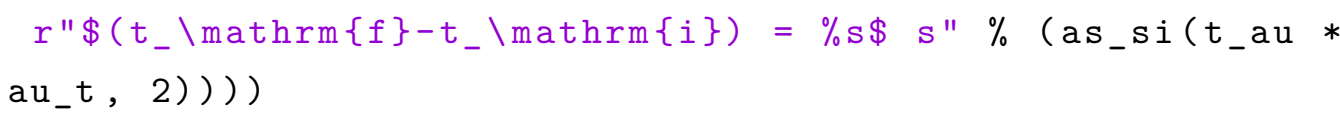




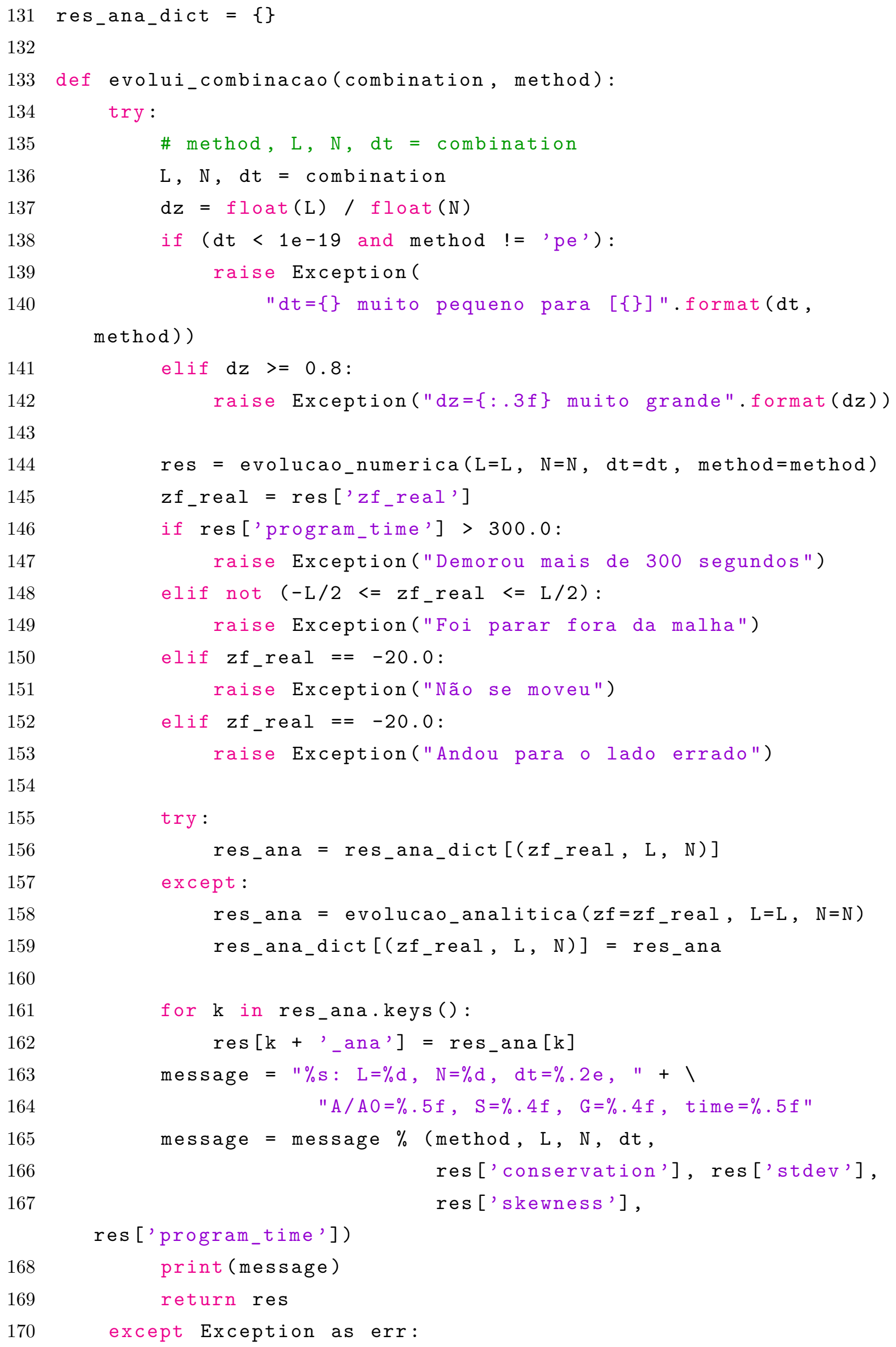


171

172

174

174

175

176

177

178

179

180

181

182

183

184

185

186

187

188

189

190

191

192

193

194

195

196

197

198

199

200

201

202 print (err)

print ("Falha em $\% \mathrm{~s}: \mathrm{L}=\% \mathrm{~d}, \mathrm{~N}=\% \mathrm{~d}, \mathrm{dt}=\% .2 \mathrm{e} " \%$ (method, L,

$N, d t)$ )

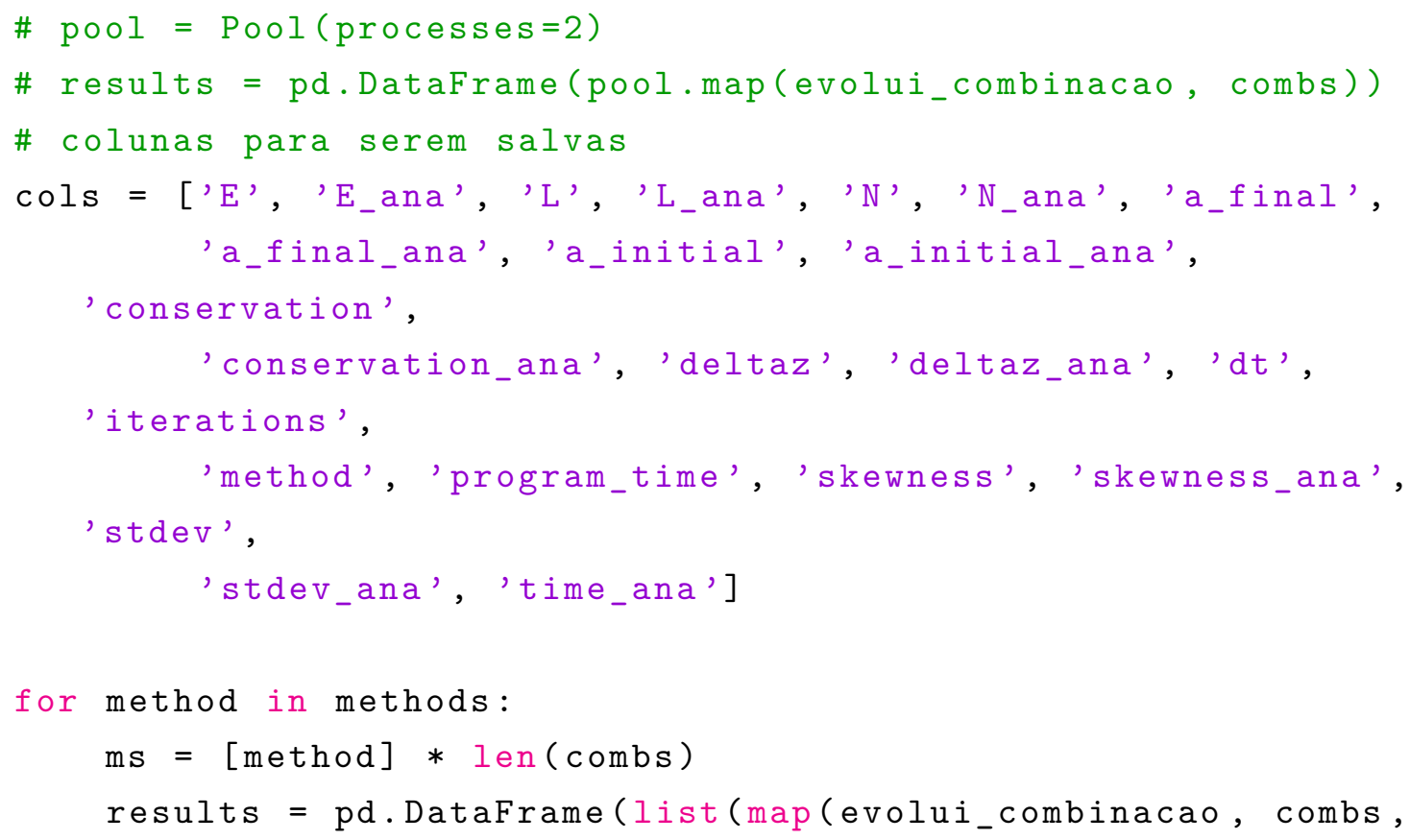


206

$207 \mathrm{fig}$, sharey=True );

208

209

210

231

$p=3$ $\mathrm{p}=\mathrm{p})[0][0]$,

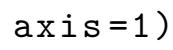

fig, $(\operatorname{ax} 1, \operatorname{ax} 2, a x 3)=$ plt.subplots $(1,3$, sharex=True,

with pd.plotting.plot_params.use('x_compat', True):

$X A=\left[\left[1 . a_{-} f i n a l, 1[\right.\right.$ 'stdev'], I['skewness' $\left.]\right]$

$\mathrm{XB}=\left[\left[1 . \mathrm{a}_{-} f i n a l_{-} \mathrm{ana}, 1 . \mathrm{stde}_{-}\right.\right.$ana, 1.skewness_ana $]$

pec ['minkowski'] = pec $\cdot \operatorname{apply}($

lambda 1: cdist (XA=XA, XB=XB, metric='minkowski',

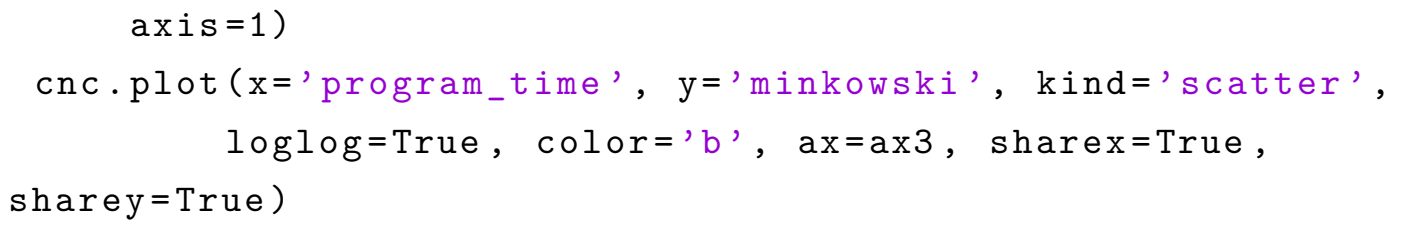


241

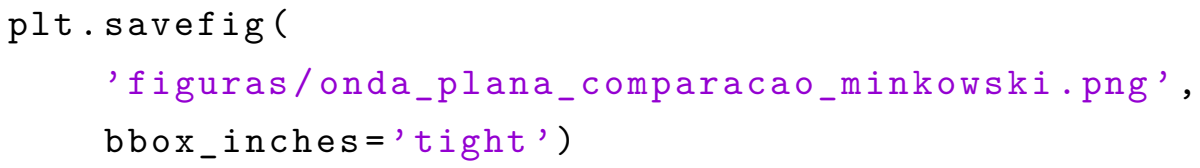

\section{A.2 Cálculo de Níveis de Energia: Oscilador Harmônico Quântico}

Neste algoritmo implementamos soluções numéricas e analíticas para obtenção das autoenergias e autoestados do oscilador harmônico quântico.

É feita uma comparação afim de identificar o esforço computacional necessário para atingir determinados níveis de precisão.

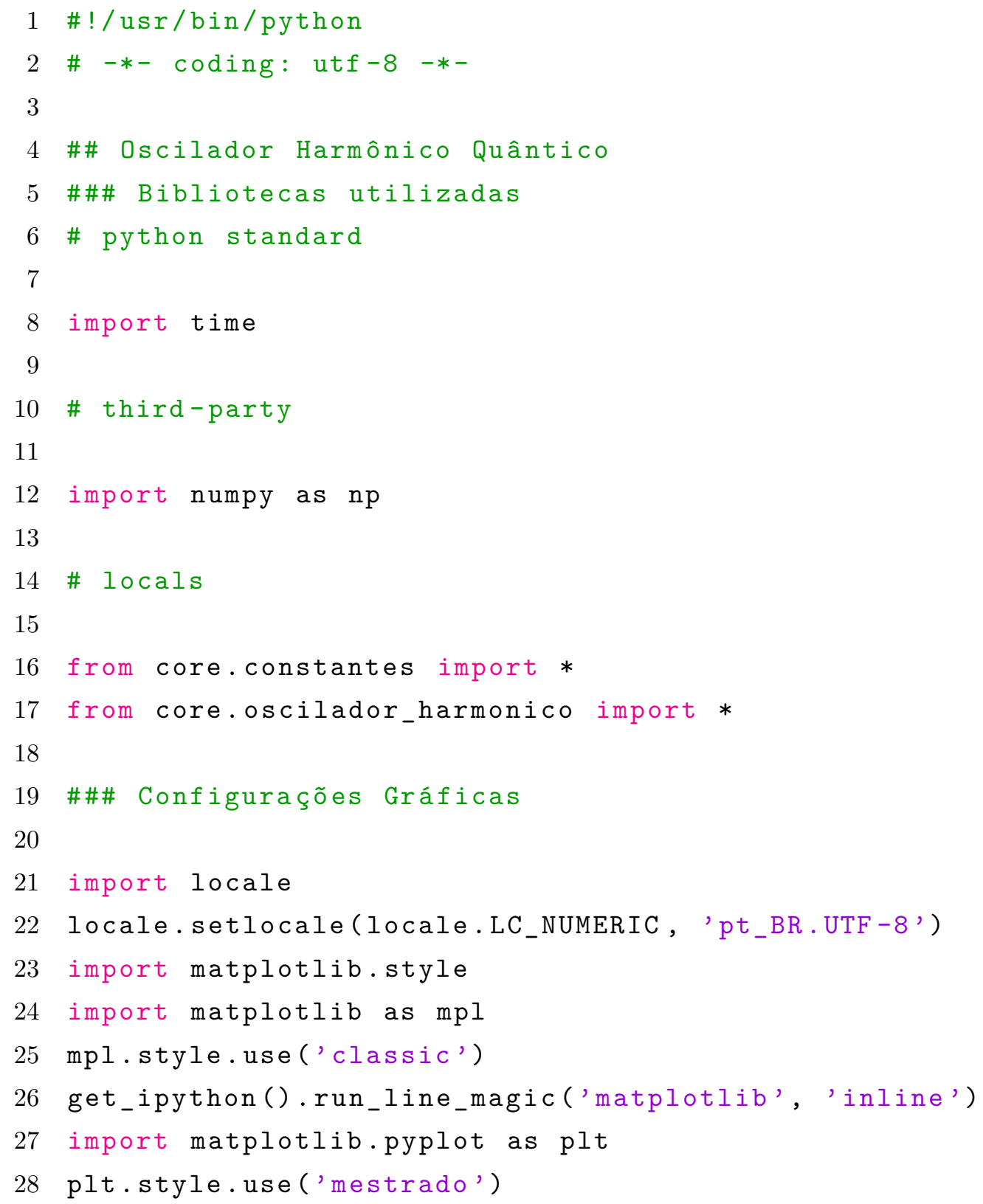


\#\# Performance do método pseudo-espectral

31

res_pe $=\{\}$

33

\# aqui coletamos

for $\max _{-}$iteracoes in [int(1e5*i) for $i$ in range $\left.(1,11)\right]$ : res_pe[max_iteracoes] = solucao_numerica $(\mathrm{N}=1024, \mathrm{nmax}=6$, method $=$ 'pe' 
67

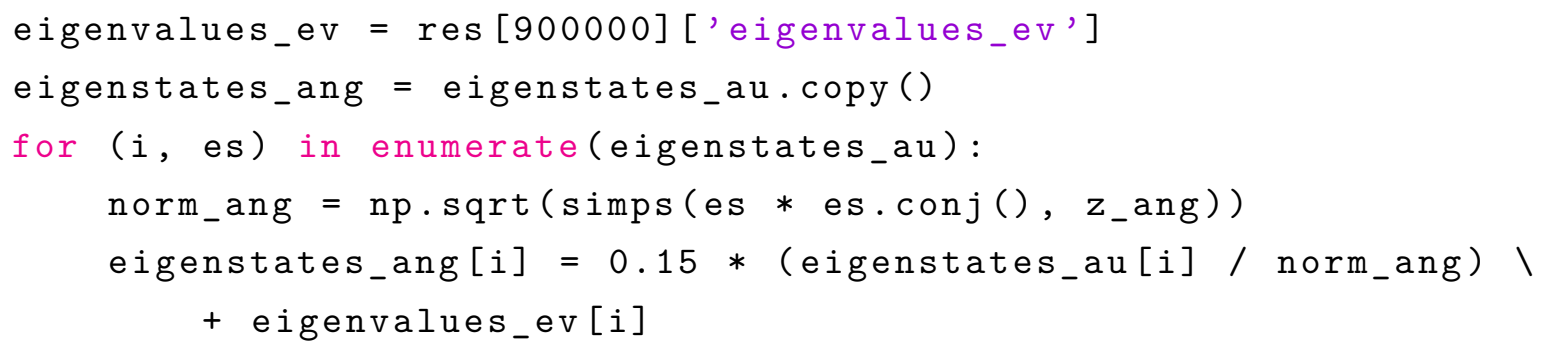


106

107

108

109

110 plt.legend ()

111

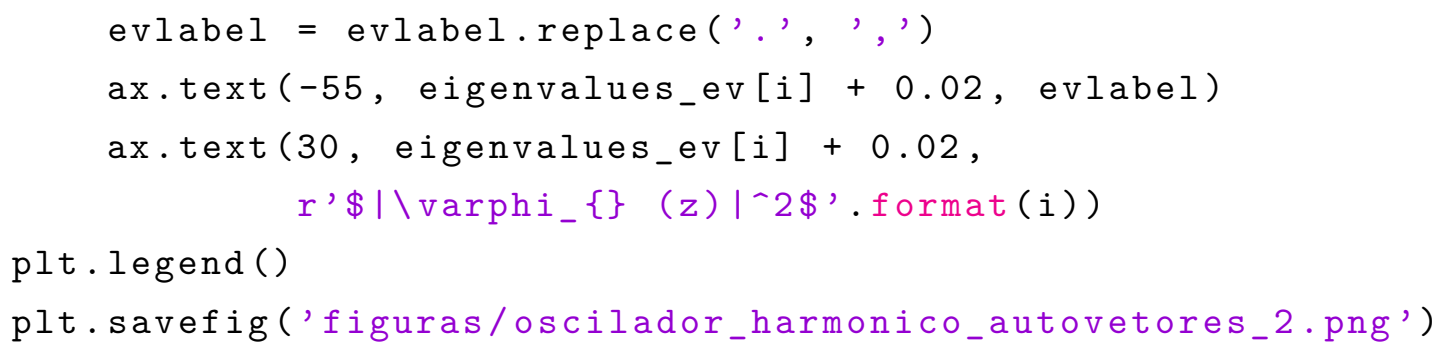

\section{A.3 Cálculo de Níveis de Energia: Poço Quântico}

Este algoritmo permite calcular autovalores de um poço quântico através do método Pseudo-Espectral e verifica a qualidade do resultado em comparação com a solução das equações transcendentais (5.24a) e (5.24b).

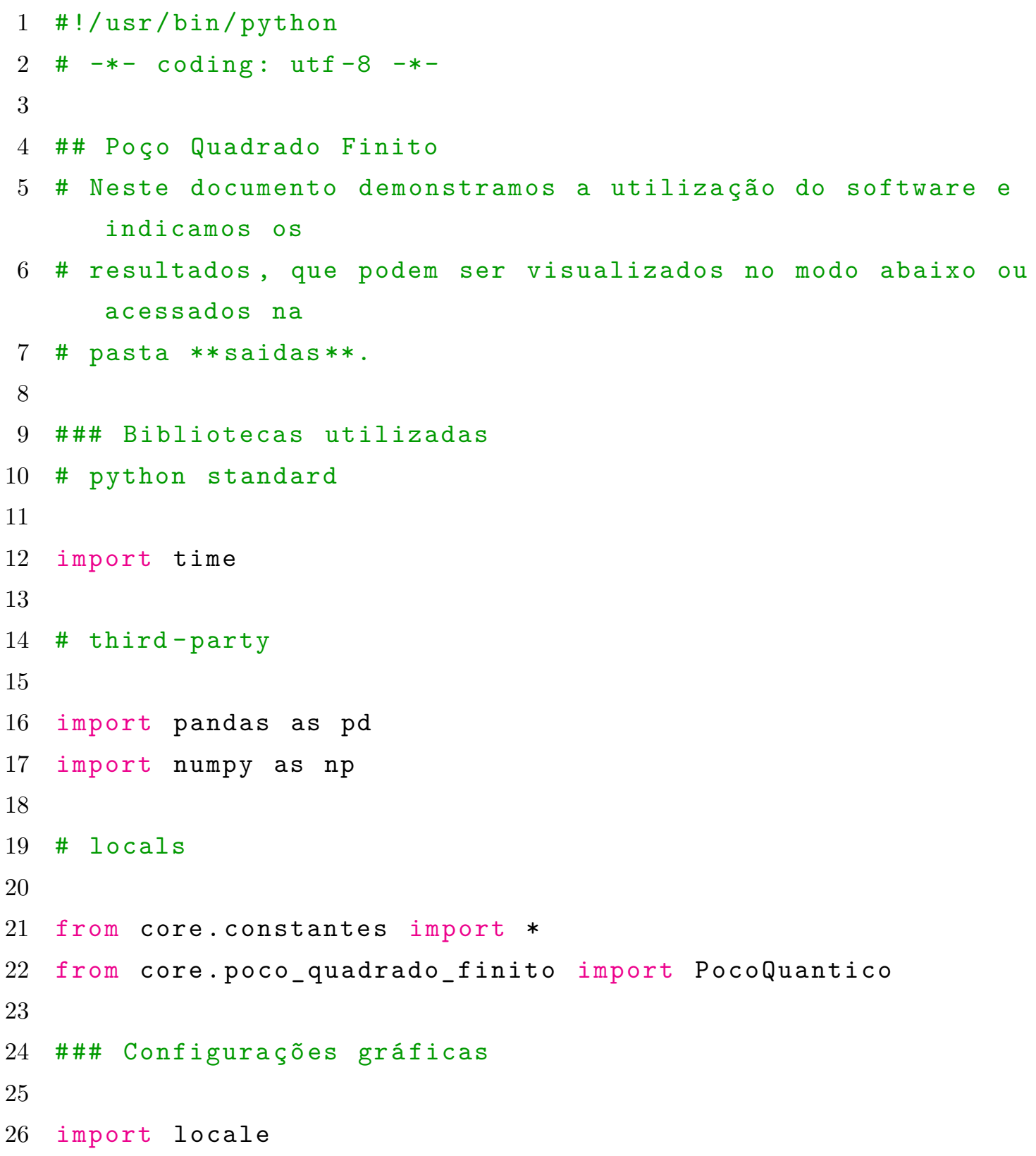


27 locale.setlocale(locale.LC_NUMERIC, 'pt_BR.UTF-8')

28 import matplotlib.style

29 import matplotlib as $\mathrm{mpl}$

30 mpl.style.use ('classic')

31 get_ipython().run_line_magic('matplotlib', 'inline')

32 import matplotlib.pyplot as plt

33 from matplotlib.ticker import MultipleLocator

34 plt.style.use ('mestrado')

35

36 \#\#\# Inicia um poço quântico

poco_100 = PocoQuantico(well_length=100.0, well_height=1.0, $\mathrm{N}=2048$,

40

41 \# Este é o potencial que vamos usar:

42

$43($ fix, ax $)=$ plt.subplots $(1,1$, figsize=(18, 6) $)$

44 ax.plot (poco_100.z_ang, poco_100.v_ev)

45 ax.set_xlabel ( $r^{\prime} z$ (A)')

46 ax.set_ylabel (r'V (eV)')

47 plt.show ()

48

49 \# que foi iniciado com as seguintes propriedades:

50 \#

51 \# - **well_length=100.0**, largura de 100.0 Angstrom

$52 \#-* * w e l l_{\text {_height }}$ - $1.0 * *$, altura de $1.0 \mathrm{eV}$

53 \# - **N=2048**, 2048 pontos na malha (discretização)

$54 \#-* * d t=1 e-19 * *$, passo de tempo de $\$ 1 \backslash$ times $10^{-}\{-18\} \$$ segundos

55 \#

56 \#\#\# Calculo dos Autoestados e Autovalores

57 \#

58 \# 0 comando abaxo solicita o cálculo dos autoestados e autovalores com

59 \# precisao de $0.01 \%$.

$60 \#$

61 \# Está precisão não é em relação ao valor analítico, mas em relação ao

62 \# valor para o qual o algoritmo convergiu (que possui um erro em 63 \# relação ao analítico).

64

65 poco_100.evolucao_imaginaria(precision=1e-4) 
66

$$
68
$$$$
69
$$$$
70
$$$$
71
$$$$
72
$$$$
73
$$$$
74
$$ 
104

105

106

107

108

109

110

111

112

113

114

115

116

117

118

119

120

121

122

123

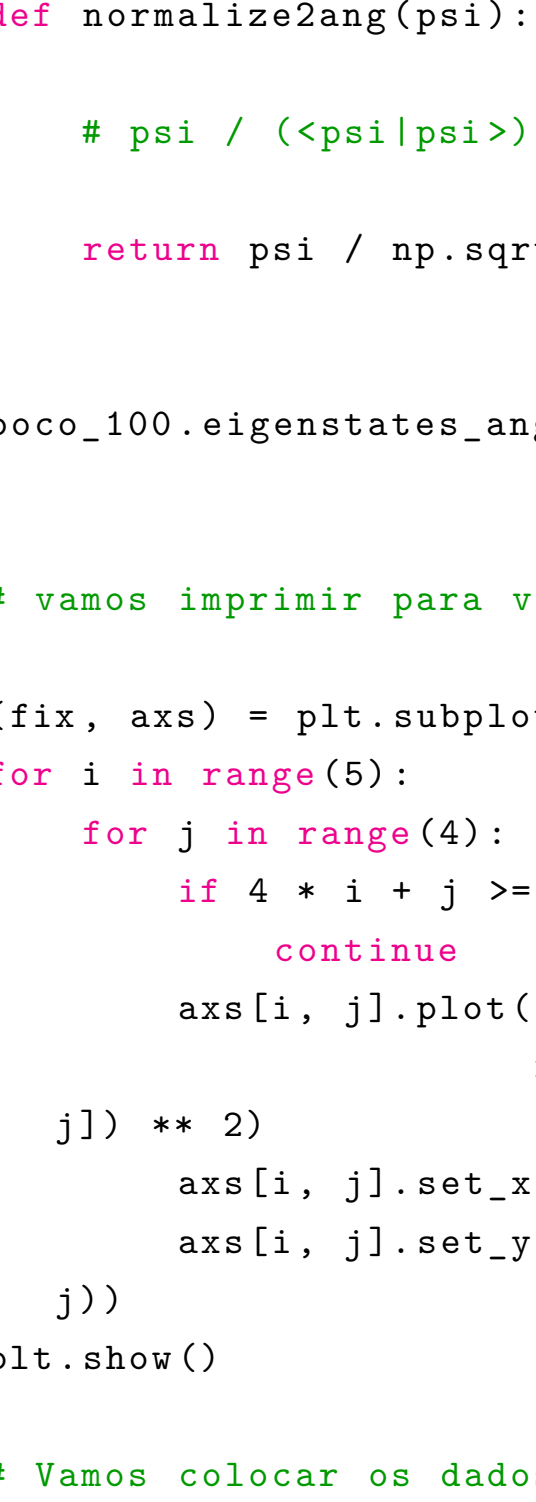

124

125

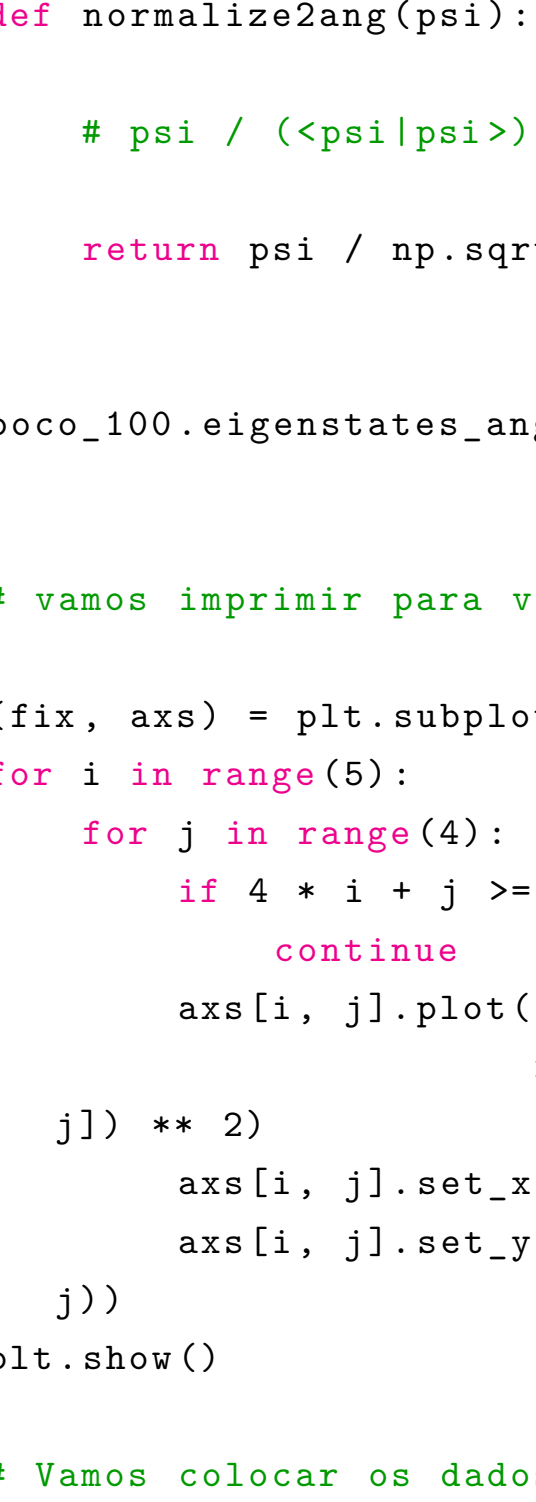

126

127

128 para

129 \# um **. csv**.

130 \# $\circ$ eixo z em Angstrom

131 \# o potencial em eV

132

133

134

135

136

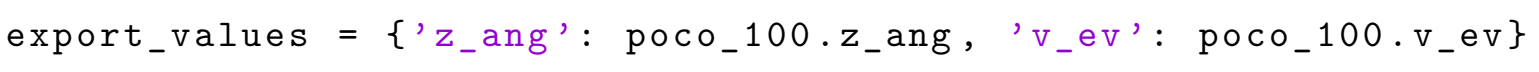

for (i, state) in enumerate(poco_100.eigenstates_ang):

export_values['psi_\%02d'\% i] = state \#psi

export_values ['psi_squared_\%d' \% i] =np.abs(state) $* * 2$ \#

| psil- 2

137

138

139

140

$$
\text { poco_100.eigenstates] }
$$


\# Nós temos também informações sobre tempo de processamento e número de

\# iterações para cada nível de energia:

perf_metrics $=$ pd.DataFrame $(\{$

'time (s)': poco_100.timers,

'iterations': poco_100.counters,

'numeric precision (\%)': $100 *$ poco_100.eigenvalues_precisions, 'analytic (eV)': poco_100.analytical_eigenvalues, 
178

179

180

181

182

183

184

185

186

187

188

189

190

191

192

193

194

195

196

197

198

199

200

201

202

203

204

205

206

207

208

209

210

211

212

213

214

215

216

217

218

219

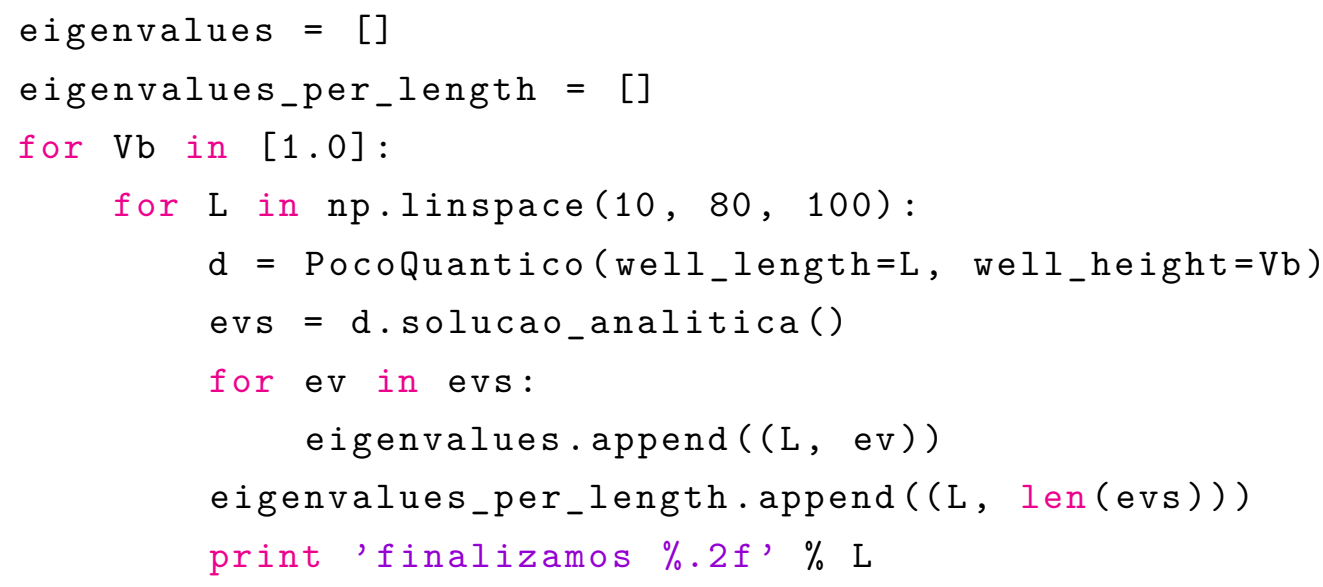


220

221

222

223

224

225

print 'Estamos em $\mathrm{L}=\% .2 \mathrm{f}, \% \mathrm{~L}$

$(\mathrm{L}$, e $)=\operatorname{tuple}(\operatorname{zip}(*$ eigenvalues $))$

$1_{-} e=p d$. DataFrame $\left(\left\{L^{\prime}:\right.\right.$ L, ' e': e $\left.\}\right)$

1_e.to_csv('saidas/poco_quantico_autovalores_numericos_por_comprimento.csv

$(L, e)=\operatorname{tuple}($ zip $(*$ eigenvalues $))$

$I_{-} e=p d$. DataFrame (\{'L': L, 'e': e $\left.\}\right)$

l_e.to_csv('saidas/poco_quantico_autovalores_numericos_por_comprimento.csv )

numeric $=1$

pd.read_csv('saidas/poco_quantico_autovalores_numericos_por_comprimento , index_col=0)

$\mathrm{ax}=$ numeric $\left[\left(\right.\right.$ numeric [' $\left.\left.\mathrm{L}^{\prime}\right]<=80\right)$ \& (numeric ['e']

$<=1.0)] \cdot$ plot.scatter $\left(x={ }^{\prime} L^{\prime}, y=e^{\prime} e^{\prime}\right)$

ax.set_xlabel( $\left.r{ }^{\prime} a(\backslash A A)^{\prime}\right)$

ax.set_ylabel ( $\left.r^{\prime} E(e V)^{\prime}\right)$

ax.set_ylim $([-0.05,1.05])$

ax.set_xlim $([8,82])$

plt.savefig('figuras/poco_quantico_numerico.png',

bbox_inches='tight')

analitic $=1$

pd.read_csv('saidas/poco_quantico_autovalores_por_comprimento.csv', index_col $=0$ )

$\mathrm{ax}=$ analitic[analitic['L'] $<=80]$.plot.scatter $\left(\mathrm{x}=\mathrm{\prime}^{\prime} \mathrm{L}^{\prime}, \mathrm{y}=\mathrm{y}^{\prime} \mathrm{e}^{\prime}\right)$

ax.set_xlabel ( $r^{\prime}$ a $\left.(\backslash A A)^{\prime}\right)$

ax.set_ylabel ( $\left.r^{\prime} E(e V)^{\prime}\right)$

ax.set_ylim $([-0.05,1.05])$

ax.set_xlim $([8,82])$

plt.savefig('figuras/poco_quantico_analitico.png',

bbox_inches='tight')

numcut $=1$

pd.read_csv('saidas/poco_quantico_autovalores_numericos_por_comprimento , index_col=0)

numcut $=$ numcut $\left[\left(\right.\right.$ numcut $\left[\mathrm{L}^{\prime}\right]<=80$ ) \& (numcut ['e'] $<=1.0$ )]

anacut $=1$

pd.read_csv('saidas/poco_quantico_autovalores_por_comprimento.csv', index_col=0)

anacut $=$ anacut $\left[\left(\operatorname{anacut}\left[{ }^{\prime} \mathrm{L}^{\prime}\right]<=80\right) \&\left(\right.\right.$ anacut $\left.\left.\left[\mathrm{e}^{\prime}\right]<=1.0\right)\right]$ 


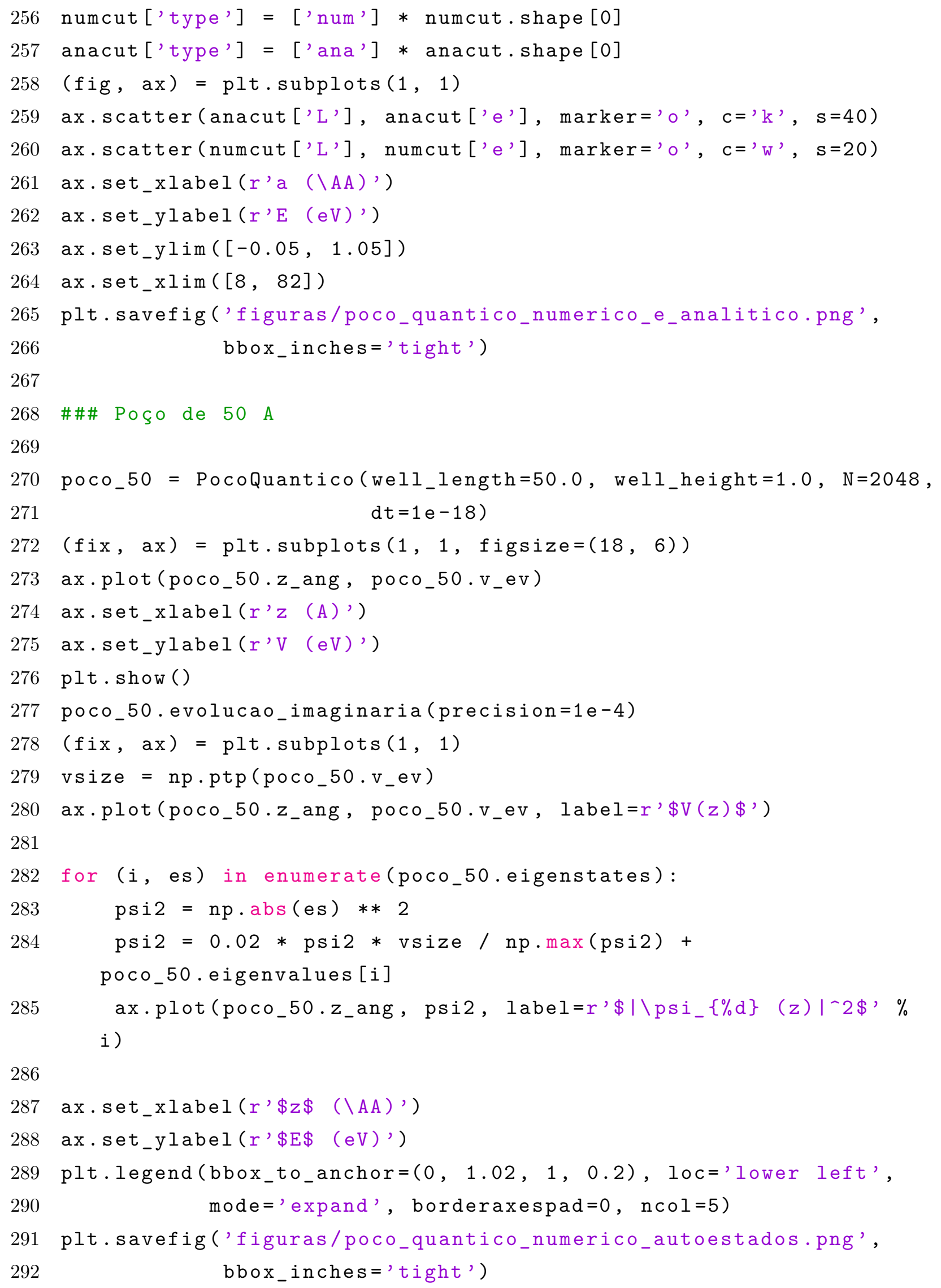




\section{A.4 Cálculo de Níveis de Energia: Poço Duplo Assimétrico}

Este algoritmo calcula utilizando o método pseudo-espectral os autovalores e autovetores do poço duplo assimétrico, introduzido por Zhirnov[43] e tratado numericamente por Feit[1].

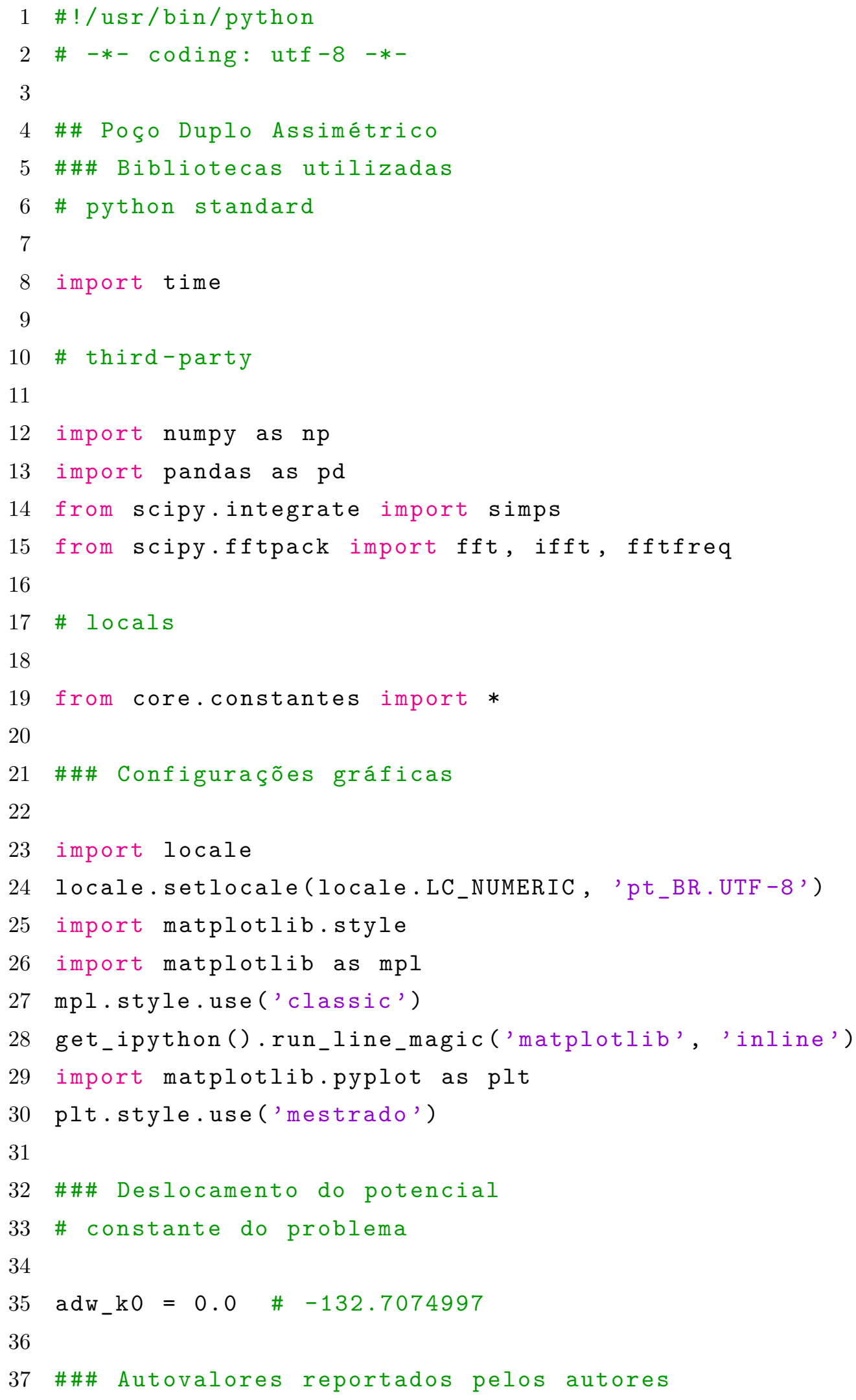


38

39

40

41

42

43

44

45

46

47

48

49

50

51

52

$f e i t \_e t \_a l=[-12.258438,-6.045418,-5.286089,-0.646627$, 0.691204 ,

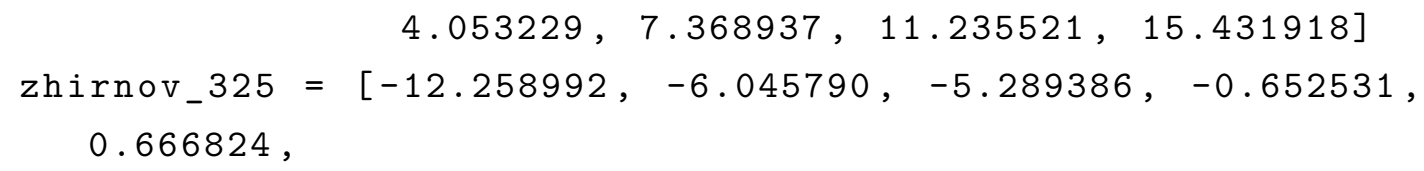




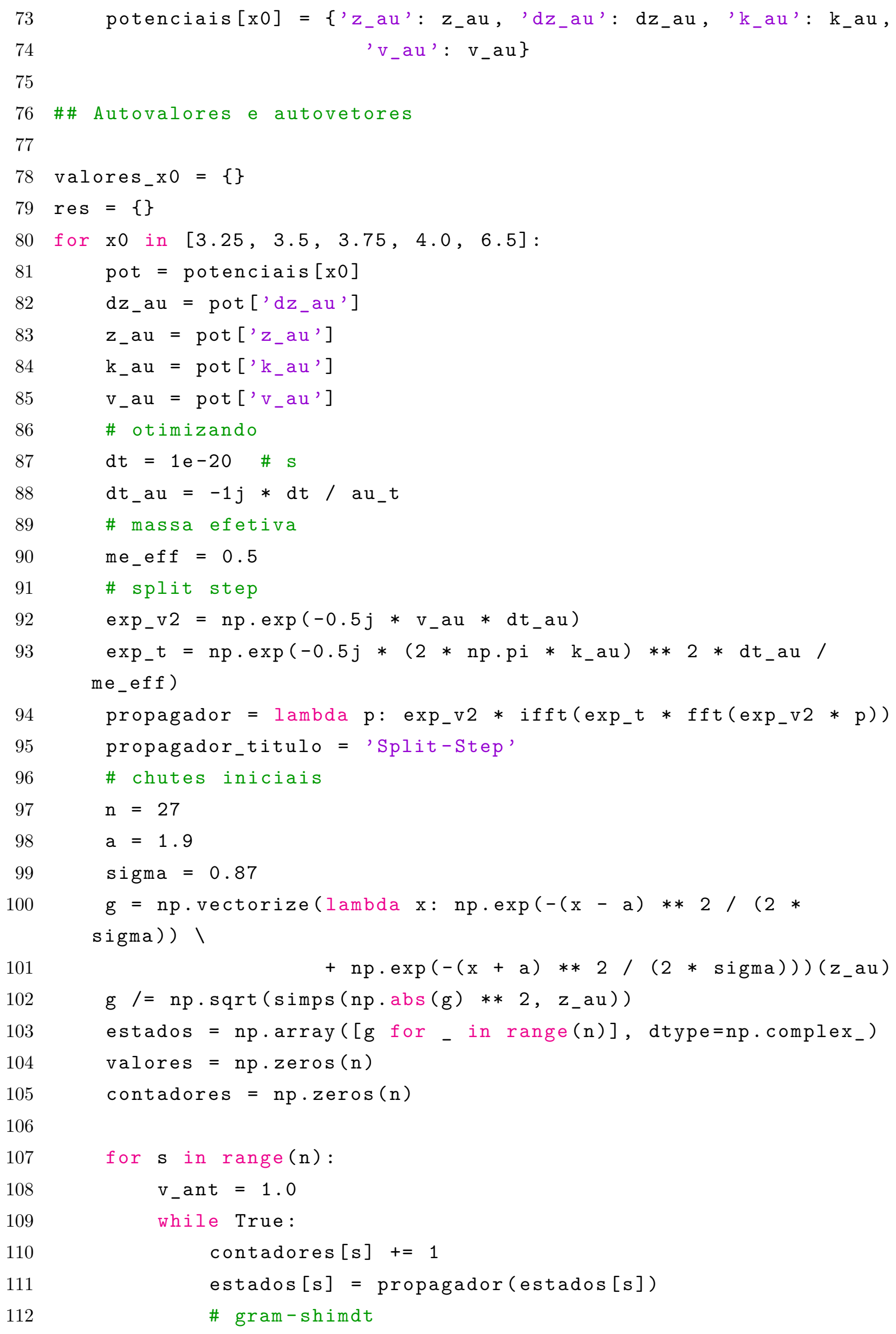


113

114

115

116

117

118

119

120

121

122

123

124

125

126

127

me_eff

128

129

130

131

132

133

134

135

136

137

138

139

140

141

142

143

144

145

146

147

148

149

150

$\left.\left.z_{-} a u\right)\right)$

\}

for $m$ in range(s):

proj $=\operatorname{simps}($ estados [s] *

np.conjugate (estados [m]),

$\left.z_{-} a u\right)$

estados [s] $-=\operatorname{proj} *$ estados [m]

\# normaliza

estados [s] /=np.sqrt ( $\operatorname{simps}(n p \cdot \operatorname{abs}(\operatorname{estados}[s]) * * 2$,

if contadores [s] $\% 1000==0$ :

\# calcula autoestados

\# derivada segunda

derivada $2=(($ estados $[s])[:-2]-2 *$

(estados $[s])[1:-1]$

$+($ estados $[\mathrm{s}])[2:]) / \mathrm{dz}_{-} \mathrm{au} * * 2$

psi $=($ estados $[s])[1:-1]$

psi_conj = np.conjugate (psi)

\# <Psi|H|Psi>

$p_{-} h_{-} p=\operatorname{simps}\left(p s i_{-} \operatorname{conj} *(-0.5 *\right.$ derivada2 $/$

\# divide por <Psi|Psi>

$\# p_{-} h_{-} p /=A$

\# print ( $\left.p_{-} h_{-} p\right)$

valores $[s]=p_{-} h_{-} p \cdot r e a l$

if $n p \cdot a b s\left(1-v a l o r e s[s] / v_{-}\right.$ant $)<1 e-7$ :

print,$\% d$ : $>>\% .8 \mathrm{e}(\% \mathrm{~d})$ ' \% (s, valores $[\mathrm{s}]$, contadores $[\mathrm{s}]$ )

break

else:

$$
v_{-} \text {ant }=\text { valores }[s]
$$

valores $\mathrm{x} 0[\mathrm{x} 0]=$ valores

res $[\mathrm{x} 0]=\{$

'autovalores': valores,

'autovetores': estados,

'contadores': contadores,

'dz_au': pot ['dz_au'],

'z_au': pot ['z_au'],

'k_au': pot ['k_au'],

'v_au': pot ['v_au'],

np.save('saidas/poco_duplo_assimetrico_full.npy', res) 


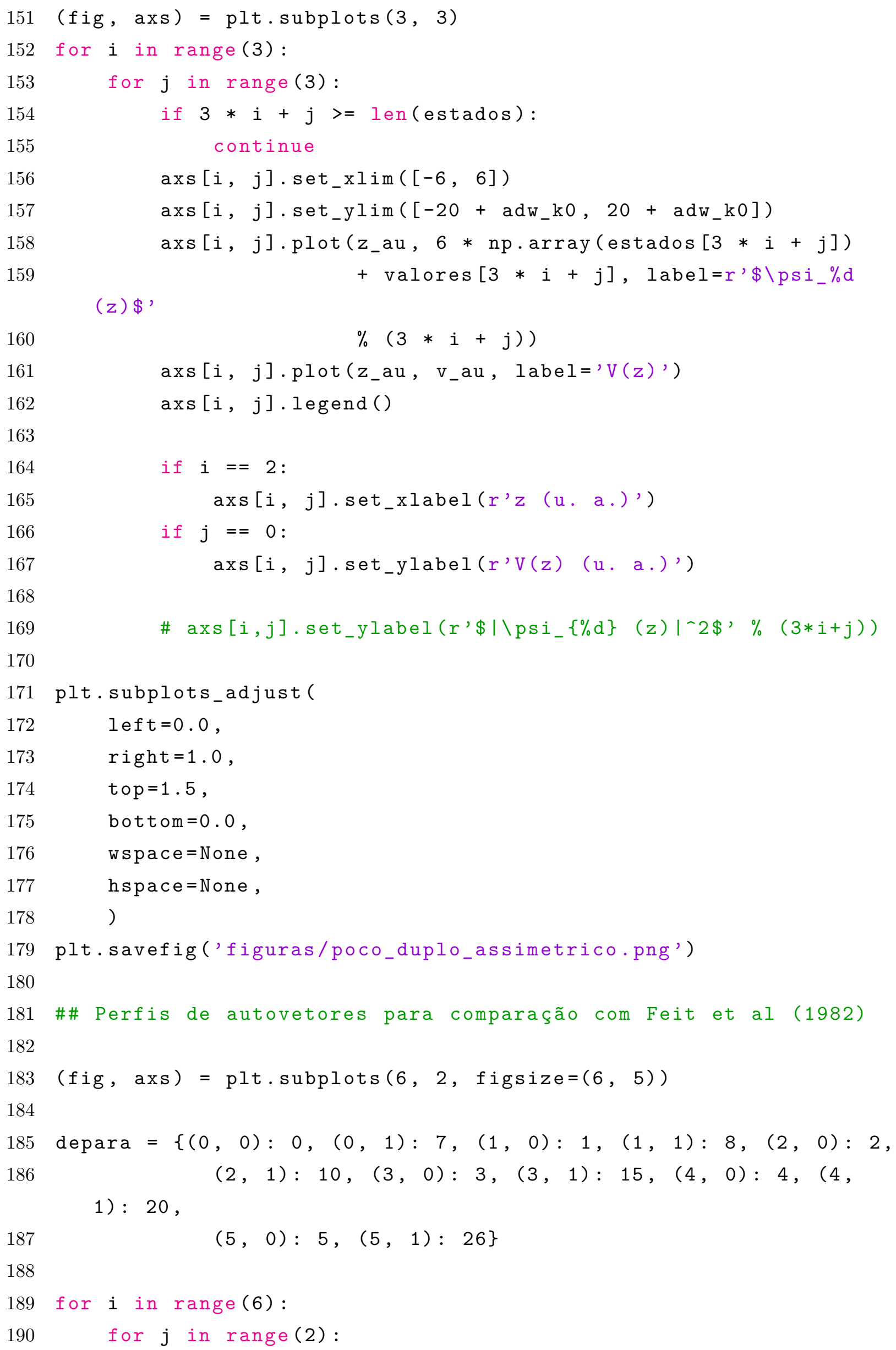


191

192

193

194

195

196

197

198

199

200

201

202

203

204

205

206

207

208

210

211

212

213

214

215

216

217

218

219

220

221

222

223

224

225

226

$$
s=\operatorname{depara}[(i, j)]
$$

axs $[i, j] \cdot p l o t\left(z_{-} a u, 6 *\right.$ np.array (estados $\left.[s]\right)+$ valores $[s]$,

$$
\text { linewidth }=0.4 \text { ) }
$$

$$
\text { label=r'\$ psi_ }\{\% d\} \quad(z) \$, \% s,
$$

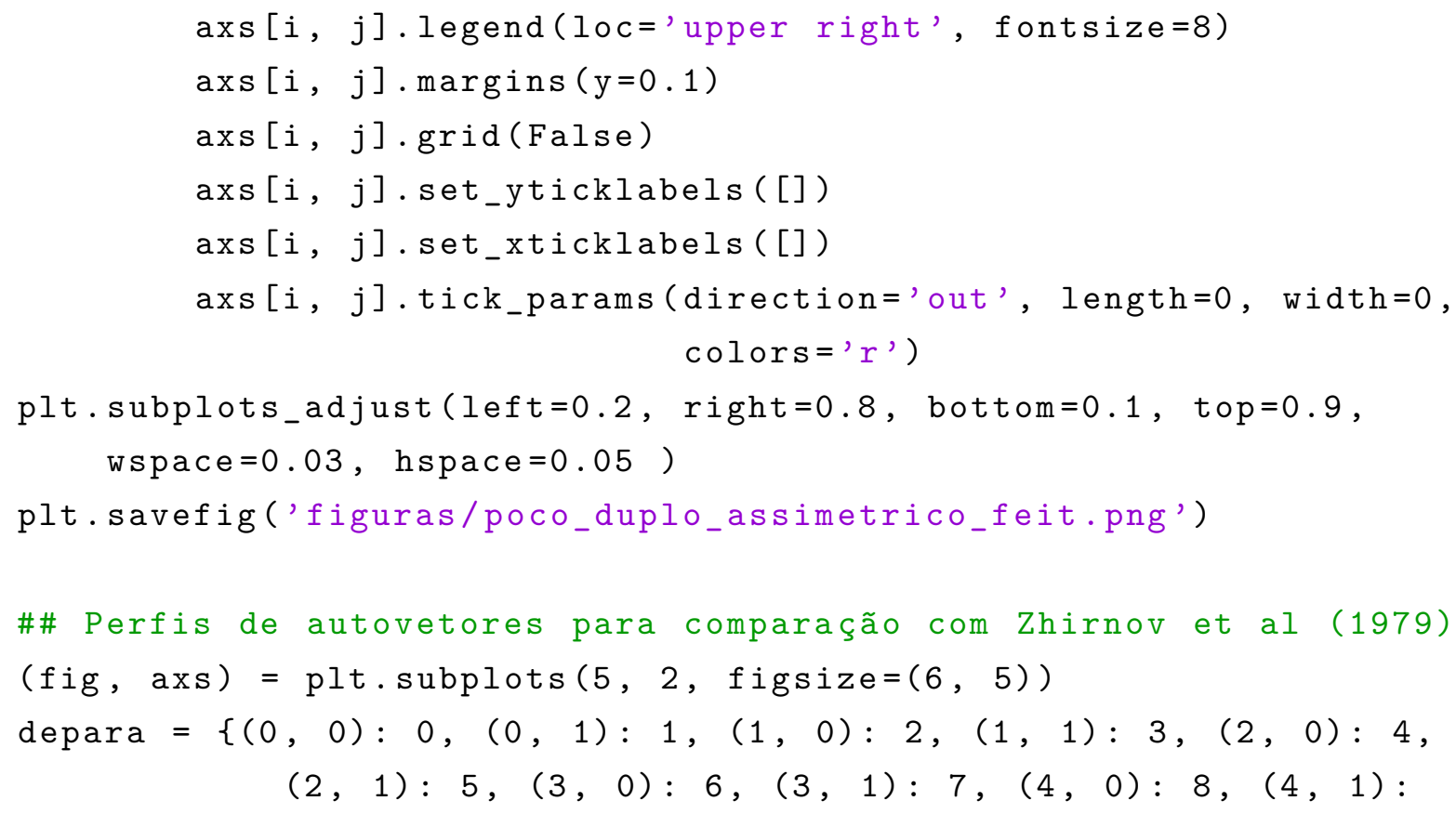




\section{A.5 Fotocorrente e Absorção em Poço Quântico de GaAs com Bar- reiras de $\mathrm{AlGaAs}$}

Este algoritmo utiliza o método da interação inversa para calcular as autoenergias e autofunções de uma estrutura do tipo poço quântico com barreiras proposta por Degani.[6] A fotocorrente e a absorção de fótons representados por um campo elétrico são calculadas evoluindo a autofunção do nível fundamental no tempo, para tanto é utilizado o método Pseudo-Espectral.

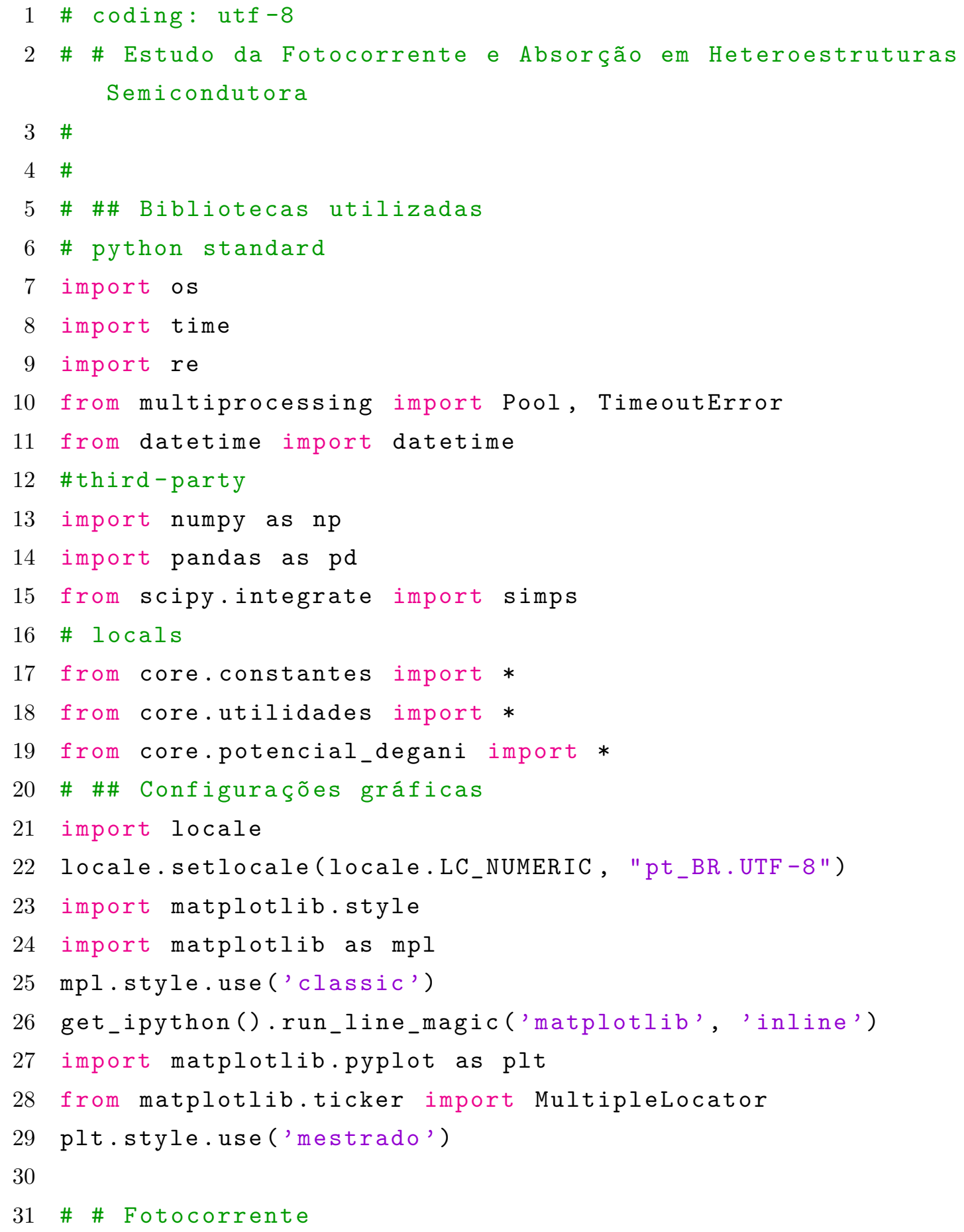




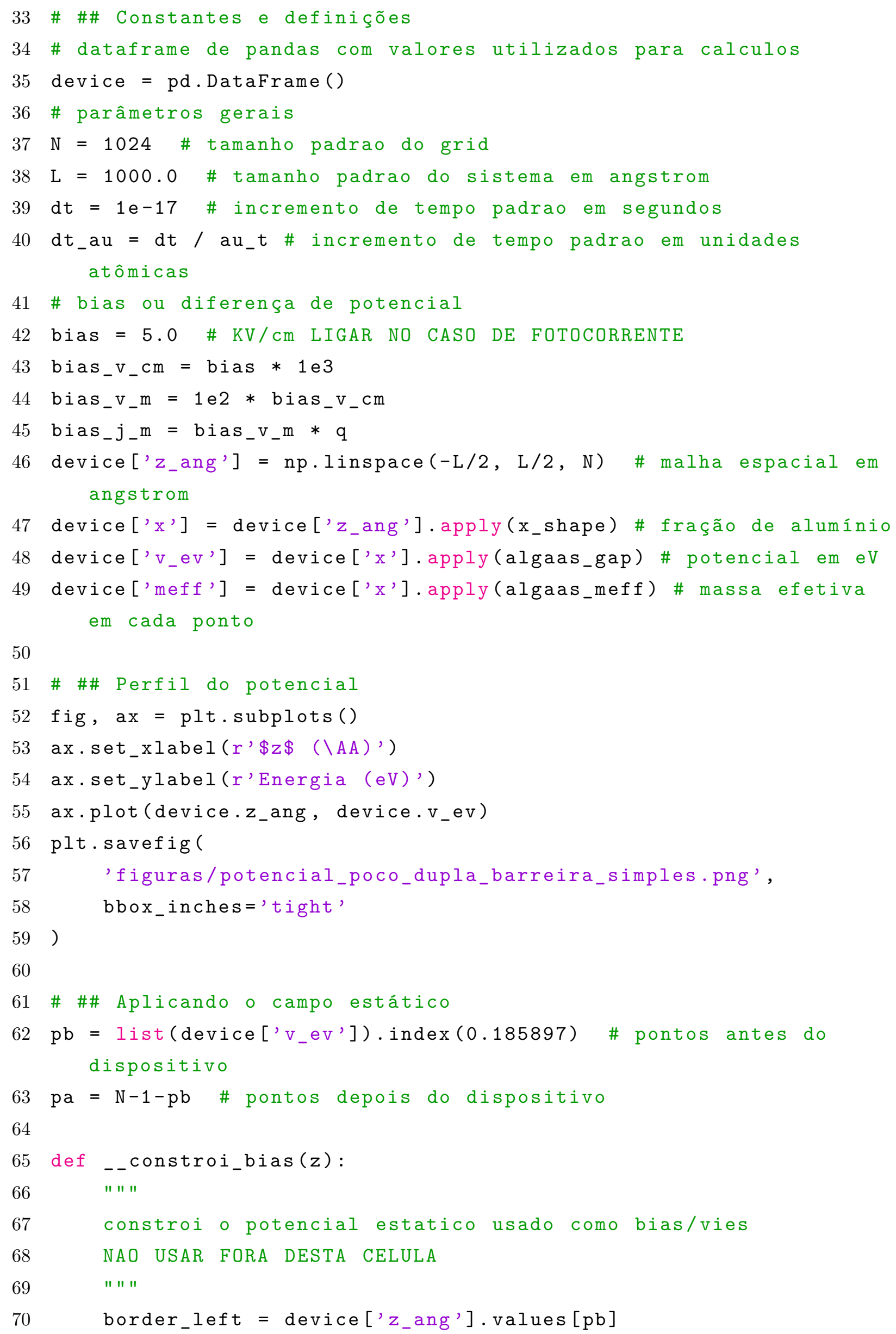


$91)$

92

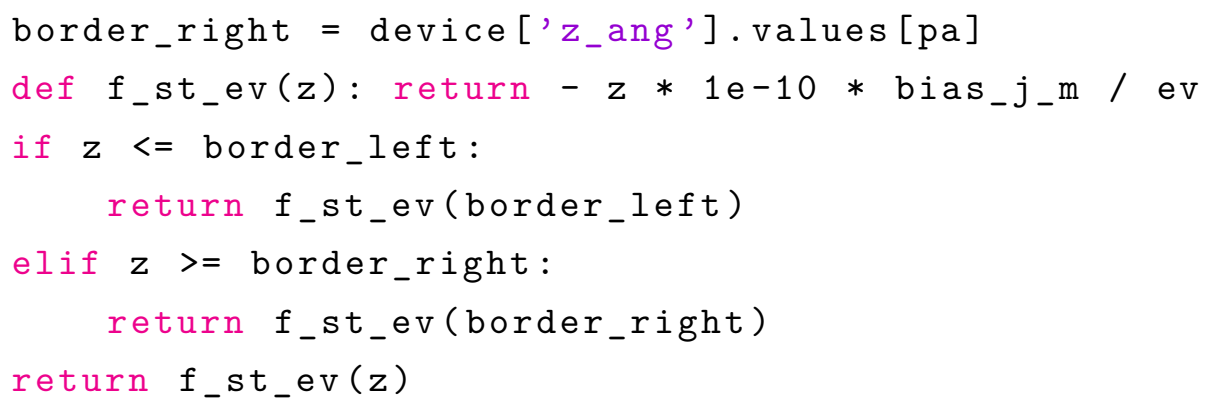


113

114

115

116

117

118

119

120

121

122

123

124

125

126

127

128

129

130

131

132

133

134

135

136

137

138

139

140

141

142

143

144

145

146

147

148

149

150

151

psi,

label=r'\$ $\left.\backslash \backslash \mathrm{Psi} \% \mathrm{~d}(\mathrm{z})\right|^{-2} 2 \backslash, \backslash, \backslash, E_{-} \% \mathrm{~d} \backslash \operatorname{approx} \% .6 \mathrm{f} \backslash, \backslash$, $\mathrm{eV} \$, \% \backslash$

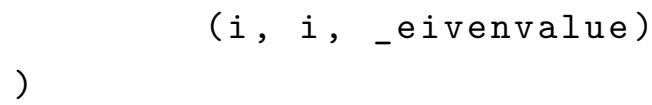


plt.savefig('figuras/absorvedor_exemplo.png', bbox_inches='tight')

153

: float=1e-12) $->$ float :

" "Calcula a fotocorrente para uma dada energia em eV (do campo eletrico)

157

158

159

160

161

162

163

164

165

166

167

168

169

170

182

183

184

185

186

187

Parâmetros

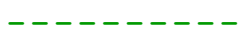

Retorno

$-------$

A fotocorrente

" " "

fosc_j_m $=$ fosc $* 1 e 2 * 1 e 3 * q \# \mathrm{KV} / \mathrm{cm}->\mathrm{J} / \mathrm{m}$

$\mathrm{T}_{-} \mathrm{au}=\mathrm{T} / \mathrm{au} \mathrm{u}_{-} \mathrm{t}$

iters $=$ int $\left(T_{-} a u / d t_{-} a u\right)$

$t_{-}$grid_au $=n p . l i n s p a c e\left(0.0, T_{-} a u\right.$, iters $)$

psi, _ = normaliza (device.z_au, device.state_0)

meff = device['meff']. values

$z_{-} a u=\operatorname{device}\left[' z_{-} a u '\right]$.values

$d z_{-} a u=z_{-} a u[1]-z_{-} a u[0]$

$\mathrm{k}_{-} \mathrm{au}=\mathrm{fftfreq}\left(\mathrm{N}, \mathrm{d}=\mathrm{dz}_{-} \mathrm{au}\right)$

$j_{-} t=n p \cdot \operatorname{zeros}($ iters $)$

def $j_{-} p(p)$ :

" " " J =1/(2 i m*) (psi* d (psi)/dz - psi d (psi*)/dz) " " " $\mathrm{dzp}=z_{-} \mathrm{au}[\mathrm{p}+1]-z_{-} \mathrm{au}[\mathrm{p}-1]$

$\operatorname{pcdp}=\operatorname{psi}[\mathrm{p}] \cdot \operatorname{conj}() *(\operatorname{psi}[\mathrm{p}+1]-\mathrm{psi}[\mathrm{p}-1]) / \mathrm{dzp}$

$\operatorname{pdpc}=\operatorname{psi}[\mathrm{p}] *(\operatorname{psi}[\mathrm{p}+1] \cdot \operatorname{conj}()-\mathrm{psi}[\mathrm{p}-1] \cdot \operatorname{conj}()) / \mathrm{dzp}$

return $((-0.5 j /(\operatorname{meff}[p])) *(p c d p-p d p c)) . r e a l$ 
191

192

193

194

195

196

197

198

199

200

201

202

203

204

205

206

207

208

209

210

211

lambda $z: \min (\operatorname{expit}((450-z) / 5), \operatorname{expit}((z+450) / 5)))$ $z 0 \_a n g=$ device['z_ang'].values [0] fosc_j = device['z_ang']. apply(lambda $z:\left(z 0_{-}\right.$ang-z) *1e-10

* fosc $\mathrm{j}_{-} \mathrm{m}$ )

$$
f o s c_{-} e v=f o s c_{-} j / e v
$$

$f o s c_{-} a u=f o s c_{-} e v / a u 2 e v$

omega_au = (energy / au2ev) / hbar_au

$v_{-} a u_{-} t i=$ device ['v_au'].values

$\exp p_{-}=n p \cdot \exp \left(-0.5 j *\left(2 * n p \cdot p i * k_{-} a u\right) * * 2 * d t_{-} a u /\right.$ meff)

$$
\begin{aligned}
& \exp v 2 h=n p \cdot \exp \left(-0.5 j * v_{-} a u_{-} t i * d t_{-} a u\right) \\
& f_{-} \operatorname{espec}=-0.5 j * f_{0} c_{-} a u * d t_{-} a u
\end{aligned}
$$

for $i, t_{-} a u$ in enumerate(t_grid_au):

$$
\exp v 2=\exp p_{-} 2 h * n p \cdot \exp \left(f_{-} \text {espec } *\right.
$$

$\left.n p \cdot \sin \left(o m e g a_{-} a u * t_{-} a u\right)\right)$

$$
\text { absorbing }
$$

$$
\text { psi }=\exp p_{-} 2 * \operatorname{ifft}\left(\exp _{-} t * \operatorname{fft}\left(\exp _{-} v 2 * p_{i} i\right)\right) *
$$

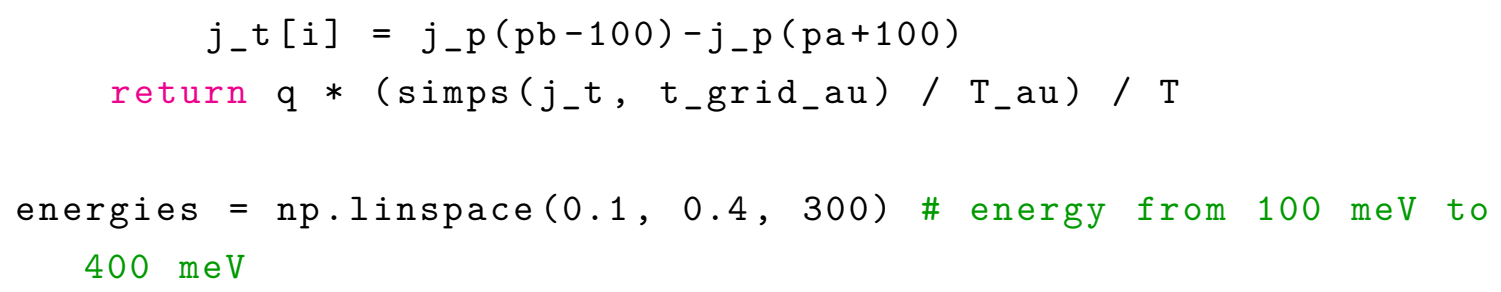


227

ax 2 .set_xticklabels $([" \% .1 \mathrm{f} " \%((\mathrm{~h} * \mathrm{c} /(\mathrm{e} * \mathrm{ev})) / 1 \mathrm{e}-6)$ for e in

$$
\text { tiny_energies]) }
$$

228

ax2.set_xlabel ( $r " \$ \backslash 1$ ambda $\$$ ( $\$ \backslash m u \$ m) ")$

229 minorLocator_x = MultipleLocator $(0.01)$

230 ax.xaxis.set_minor_locator (minorLocator_x)

231 ax.grid(which = 'minor')

232 plt.savefig('figuras/fotocorrente_degani.png',

bbox_inches='tight')

233

234

235

236 \# \#\# Definindo novamente o sistema

$237 \mathrm{~N}=1024$ \# tamanho padrao do grid

238

$L=500.0$ \# tamanho padrao do sistema em angstrom

239

$d t=1 e-17$ \# incremento de tempo padrao em segundos

240

$d t_{-} a u=d t / a u_{-} t$ \# incremento de tempo padrao em unidades

atômicas

241

242

243

\# dataframe de pandas com valores utilizados para calculos

device $=$ pd. DataFrame ()

device ['z_ang'] = np.linspace (-L/2, L/2, N) \# malha espacial em angstrom

244 device['z_au'] = device['z_ang']. apply(lambda z: z / au2ang)

245 device [' $\left.\mathrm{x}^{\prime}\right]=$ device ['z_ang'].apply ( $\mathrm{x}_{-}$shape $)$

246 device ['v_ev'] = device['x'].apply(algaas_gap)

247 device['meff'] = device['x'].apply(algaas_meff)

$248 \mathrm{pb}=$ list $($ device ['v_ev']). index $(0.185897)$ \# pontos antes do dispositivo

249 pa $=\mathrm{N}-1-\mathrm{pb} \quad \#$ pontos depois do dispositivo

250 \# não precisa de bias

251 bias $=0.0$

252 bias_v_cm = bias * 1 e3

253 bias_v_m $=1$ e2 * bias_v_cm

254 bias_j_m = bias_v_m $*$ q

255 \# mas precisa de campo elétrico

$256 \mathrm{fosc}=5.0 \# \mathrm{KV} / \mathrm{cm}$

$257 \mathrm{fosc}_{-} \mathrm{j}_{-} \mathrm{m}=\mathrm{fosc} * 1 \mathrm{e} 2 * 1 \mathrm{e} 3 * \mathrm{q} \# \mathrm{KV} / \mathrm{cm} \rightarrow \mathrm{J} / \mathrm{m}$

$258 \mathrm{fosc}_{-} \mathrm{au}=\mathrm{fosc}_{-} \mathrm{j}_{-} \mathrm{m} / \mathrm{au} \mathrm{u}_{-} \mathrm{ef}$

$259 z_{-} a u=d e v i c e \cdot z_{-} a u$

$260 \mathrm{Hpo}=z_{-}$au $*$ fosc_au

261

262

263

def__constroi_bias (z):

" " " 
264

286

constroi o potencial estatico usado como bias/vies NAO USAR FORA DESTA CELULA

" " "

$$
\text { border_left = device['z_ang'].values[pb] }
$$$$
\text { border_right = device['z_ang']. values[pa] }
$$

$$
\text { def } f_{-} s_{-} e v(z): \operatorname{return}-(z * 1 e-10) *\left(b i a s_{-} j_{-} m\right) / e v
$$


304

305

306

307

308

309

310

311

312

313

314

315

316

317

318

319

320

321

322

ax.grid (True)

ax.legend (loc='upper center', bbox_to_anchor $=(0.5,-0.1)$, shadow=True, $\mathrm{ncol}=2$ )

plt.savefig('figuras/double_barrier_quantum_well_degani_sem_vies.png', bbox_inches='tight')

\# \#\# Absorção com regra de ouro de Fermi

$z_{-} a u=$ device. $z_{-} a u \cdot v a l u e s$

eigenstates = res ["autovetores"]

eigenvalues = res ["autovalores"] *au2ev

psio = eigenstates $[0] \cdot \operatorname{copy}()$

psio_au, _ = normaliza( $z_{-} a u$, psio)

e0_au = eigenvalues [0] / au2ev

psi1 = eigenstates [1]. copy()

psi1_au, _ = normaliza( $z_{-} a u$, psi1)

e1_au = eigenvalues [1] / au2ev

psi2 = eigenstates [2]. copy ()

psi2_au, _ = normaliza( $z_{-} a u$, psi2)

e2_au = eigenvalues[2] / au2ev

$w 10_{-} a u=\left(e 1_{-} a u-e 0_{-} a u\right) / h b a r_{-} a u$

$w 20_{-} a u=\left(e 2_{-} a u-e 0_{-} a u\right) / h b a r_{-} a u$

p1_Hpo_p0_au $=\operatorname{simps}\left(p s i 0_{-} a u \cdot \operatorname{conj}() * H_{p o} *\right.$ psi1_au, z_au $)$

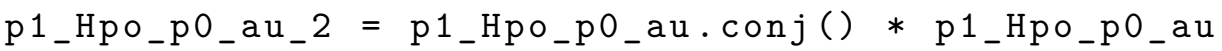

p2_Hpo_pO_au $=\operatorname{simps}\left(p s i O_{-} a u \cdot \operatorname{conj}() * H_{p o} *\right.$ psi2_au, $\left.z_{-} a u\right)$

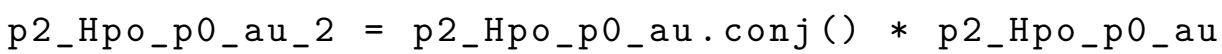

energies $=n p . l i n s p a c e(0.1,0.4,1000) \# \mathrm{ev}$

energies_au = energies / au2ev

omegas_au = energies_au / hbar_au

to $=1 \mathrm{e}-12$

to_au $=t 0 / a u_{-} t$

pcte10 $=$ t0_au**2 $*$ p1_Hpo_pO_au_2

pcte20 = to_au**2 * p2_Hpo_pO_au_2

def absorption10( $\left.w_{-} a u\right)$ :

$\mathrm{a} 1=\left(\mathrm{np} \cdot \sin \left(\left(\mathrm{w} 10_{-} \mathrm{au}-\mathrm{w}_{-} \mathrm{au}\right) * \mathrm{t} 0_{-} \mathrm{au} / 2\right)\right) /\left(\left(\mathrm{w} 10_{-} \mathrm{au}-\mathrm{w}_{-} \mathrm{au}\right) * t 0_{-} \mathrm{au} / 2\right)$

$a 2=\left(n p \cdot \sin \left(\left(w 10_{-} a u+w_{-} a u\right) * t 0_{-} a u / 2\right)\right) /\left(\left(w 10_{-} a u+w_{-} a u\right) * t 0_{-} a u / 2\right)$

a3 $=2.0 * n p \cdot \cos \left(w_{-} a u * t 0_{-} a u\right) * a 1 * a 2$

return $($ pcte10 * (a1**2+a $2 * * 2+a 3))$.real

def absorption20( $\left.w_{-} a u\right)$ :

$\mathrm{a} 1=\left(\mathrm{np} \cdot \sin \left(\left(\mathrm{w} 20_{-} \mathrm{au}-\mathrm{w}_{-} \mathrm{au}\right) * t 0_{-} \mathrm{au} / 2\right)\right) /\left(\left(\mathrm{w} 20_{-} \mathrm{au}-\mathrm{w}_{-} \mathrm{au}\right) * t 0_{-} \mathrm{au} / 2\right)$ 
344

$$
\begin{aligned}
& \text { a2 }=\left(n p \cdot \sin \left(\left(w 20_{-} a u+w_{-} a u\right) * t 0_{-} a u / 2\right)\right) /\left(\left(w 20_{-} a u+w_{-} a u\right) * t 0_{-} a u / 2\right) \\
& \text { a3 }=2 \cdot 0 * n p \cdot \cos \left(w_{-} a u * t 0_{-} a u\right) * a 1 * a 2
\end{aligned}
$$$$
\text { return }(\text { pcte } 20 *(a 1 * * 2+a 2 * * 2+a 3)) \text {.real }
$$

fig, $a x=p l t \cdot \operatorname{subplots}()$

ax.set_xlabel (r'Energia (eV)')

ax.set_ylabel ( $r^{\prime}$ Absor $\backslash c\{c\} \backslash \sim\{a\} \circ$ (unid. arb.)')

ax. semilogy

omegas_au*au2ev, np.vectorize(absorption10)(omegas_au).real, label=r'\$ $\backslash$ psi_o(z) \rightarrow \psi_1(z)\$')

ax. semilogy (

omegas_au*au2ev, np.vectorize(absorption20)(omegas_au).real, label=r'\$ $\backslash$ psi_o(z) \rightarrow $\left.\backslash p s i_{-} 2(z) \${ }^{\prime}\right)$

ax.grid (True)

plt.legend ()

plt.savefig(

'figuras/absorcao_poco_dupla_barreira_fermi_golden_rule.png', bbox_inches='tight')

\# \#\#\# Absorção com evolução temporal

def absorption_time_evolution (energy, fosc=5.0, T=1e-12): $\mathrm{fosc}_{-} \mathrm{j}_{-} \mathrm{m}=\mathrm{fosc} * 1 \mathrm{e} 2 * 1 \mathrm{e} 3 * \mathrm{q} \# \mathrm{KV} / \mathrm{cm} \rightarrow \mathrm{J} / \mathrm{m}$ $\mathrm{T}_{-} \mathrm{au}=\mathrm{T} / \mathrm{au} \mathrm{u}_{-} \mathrm{t}$ iters $=\operatorname{int}\left(T_{-} a u / d t_{-} a u\right)$ $t_{-}$grid_au $=n p . l i n s p a c e\left(0.0, T_{-} a u\right.$, iters) meff $=$ device['meff'].values $z_{-} a u=$ device['z_au'].values $\mathrm{dz}_{-} \mathrm{au}=\mathrm{z}_{-} \mathrm{au}[1]-z_{-} \mathrm{au}[0]$

$$
\mathrm{k}_{-} \mathrm{au}=\operatorname{fftfreq}\left(\mathrm{N}, \mathrm{d}=\mathrm{d} z_{-} \mathrm{au}\right)
$$

$\mathrm{d} 1 \mathrm{r}=\mathrm{np} \cdot \mathrm{zeros}$ (iters)

$\mathrm{d} 1 \mathrm{i}=\mathrm{np} \cdot \mathrm{zeros}$ (iters)

$\mathrm{d} 2 \mathrm{r}=\mathrm{np} \cdot \mathrm{zeros}$ (iters)

$\mathrm{d} 2 \mathrm{i}=\mathrm{np} \cdot \operatorname{zeros}(\mathrm{iters})$

psi $=n p \cdot \operatorname{array}($ device.state_o, dtype=np.complex_ $)$

psi $/=\operatorname{np} \cdot \operatorname{sqrt}\left(\operatorname{simps}\left(\operatorname{psi} * \operatorname{psi} \cdot \operatorname{conj}(), \operatorname{device\cdot z_{-}au))}\right.\right.$

psi1 = eigenstates [1]. $\operatorname{copy}()$

psi1_au $=\operatorname{psi1} / \operatorname{np} \cdot \operatorname{sqrt}\left(\operatorname{simps}\left(\operatorname{psi1} \cdot \operatorname{conj}() * \operatorname{psi1}, z_{-} a u\right)\right)$ e1_au = eigenvalues[1] / au2ev psi2 = eigenstates [2]. $\operatorname{copy}()$ psi2_au $=\operatorname{psi2} / \operatorname{np} \cdot \operatorname{sqrt}\left(\operatorname{simps}\left(\operatorname{psi2} \cdot \operatorname{conj}() * \operatorname{psi2}, z_{-} a u\right)\right)$ e2_au = eigenvalues[2] / au2ev absorbing = device['z_ang']. apply ( 
417

418

419

420

421

424

425

426

427

428

429

430

431

432

433

434

435

436

437

438

439

440

441

442

443

444

445

446

447

448

449

450

451

452

453

energies $=n p .1$ inspace $(0.1,0.4$, 300) \# energy from $100 \mathrm{meV}$ to

$400 \mathrm{meV}$

def get_abs (energy):

$\mathrm{pc}=$ absorption_time_evolution (energy=energy)

now $=$ datetime.now ().strftime ( $\% \mathrm{Y}-\% \mathrm{~m}-\% \mathrm{~d} \% \mathrm{H}: \% \mathrm{M}: \% \mathrm{~S} ")$

print (" $[\% \mathrm{~s}]>$ Energy: \%.6f eV, Abs: \%.6e " \% (now, energy, $\mathrm{pc})$ )

return pc

pool $=\operatorname{Pool}($ processes $=4)$

absorption_values = pool.map (get_abs, energies)

abs1 = [a[0] for a in absorption_values]

abs2 = [a[1] for a in absorption_values]

abs3 = [a[2] for a in absorption_values]

abs4 = [a[3] for a in absorption_values]

np.savez ("saidas/absorption_1_cos_j", energies, abs1)

np.savez ("saidas/absorption_2_cos_j", energies, abs2)

np.savez ("saidas/absorption_1_cos", energies, abs3)

np.savez ("saidas/absorption_2_cos", energies, abs4)

loads1 = np.load ("saidas/absorption_1_cos_j.npz")

loads2 = np.load ("saidas/absorption_2_cos_j.npz")

energies = loads1['arr_0']

$\operatorname{abs} 1=\operatorname{loads1}\left[\operatorname{larr}_{-}{ }^{\prime}\right.$ ]

$\operatorname{abs} 2=\operatorname{loads} 2\left[\operatorname{larr}_{-}{ }^{\prime}\right.$ ]

fig, $a x=$ plt.subplots ()

ax.set_xlabel ( $\left.r^{\prime} \$ E \$(e V)^{\prime}\right)$

ax.set_ylabel (r'Absor $\backslash c\{c\} \backslash \sim\{a\} o$ (unid. arb.) ')

ax.semilogy (energies, energies* (np.abs (abs1)+np.abs (abs2)))

plt.savefig ('figuras/absorcao_degani.png', bbox_inches='tight')

$\mathrm{df}=$ pd.read_csv('saidas/absorption.csv')

energy $=d f$.energy.values

$d 1 r=d f \cdot d 1 r \cdot v a l u e s$

$\mathrm{d} 2 \mathrm{r}=\mathrm{df} \cdot \mathrm{d} 2 \mathrm{r} \cdot \mathrm{values}$

$\mathrm{d} 1 \mathrm{i}=\mathrm{df} \cdot \mathrm{d} 1 \mathrm{i} \cdot \mathrm{values}$

$d 2 i=d f \cdot d 2 i \cdot v a l u e s$

fig, $a x=$ plt.subplots ()

ax.set_xlabel ( $\left.r^{\prime} \$ E \$(e V)^{\prime}\right)$

ax.set_ylabel (r'Absor $\backslash c\{c\} \backslash \sim\{a\} o$ (unid.arb.) ')

\#ax.semilogy (energy, energy*(np.abs(d1i)), label=r'\$ p psi_0

\rightarrow \psi_1\$')

454 \#ax.semilogy(energy, energy*(np.abs(d2i)), label=r'\$ $\backslash$ psi_0

$\backslash$ rightarrow $\backslash$ psi_2\$') 


\section{A.6 Efeitos da Tensão e Segregação do In em Poços Quânticos de $\ln \mathrm{GaAs} / \mathrm{GaAs}$}

Este algoritmo leva em conta efeitos de tensão e segregação para calcular as energias de recombinação entre elétrons e buracos durante medidas de PL em poços quânticos de InGaAs sobre GaAs.

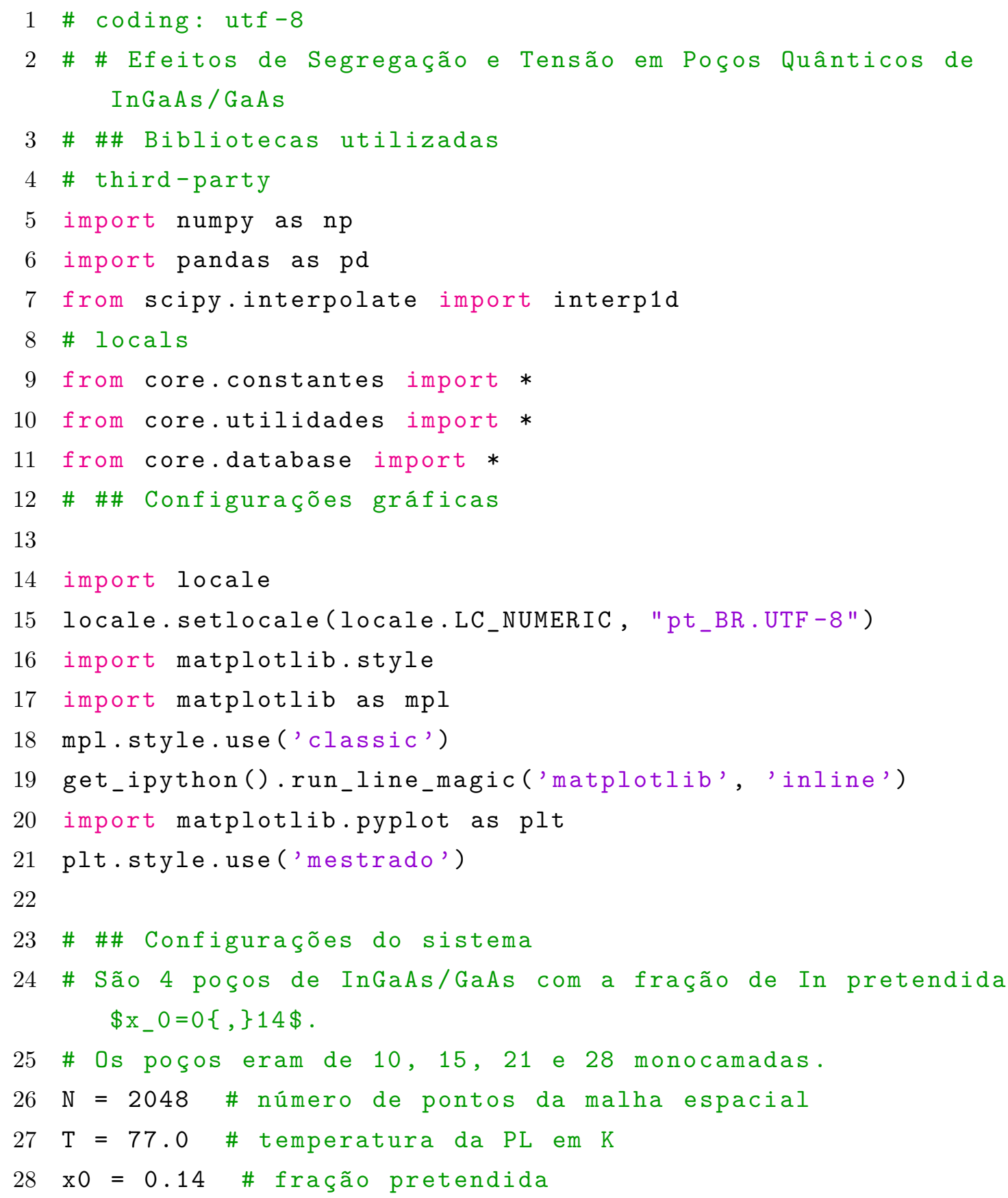




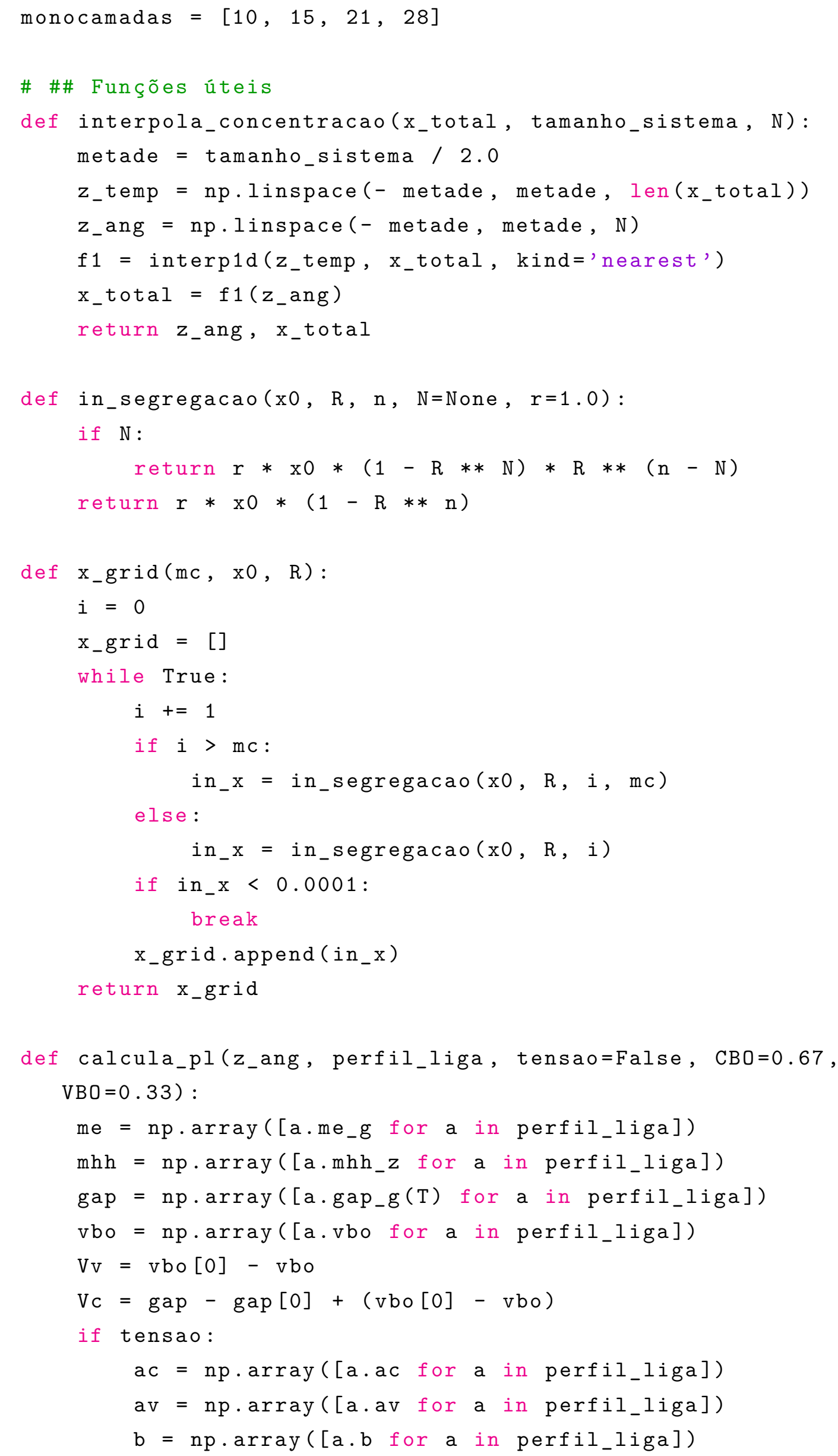


82

83

99$$
100
$$$$
101
$$$$
102
$$

103

104

105

106

$107\}$

108

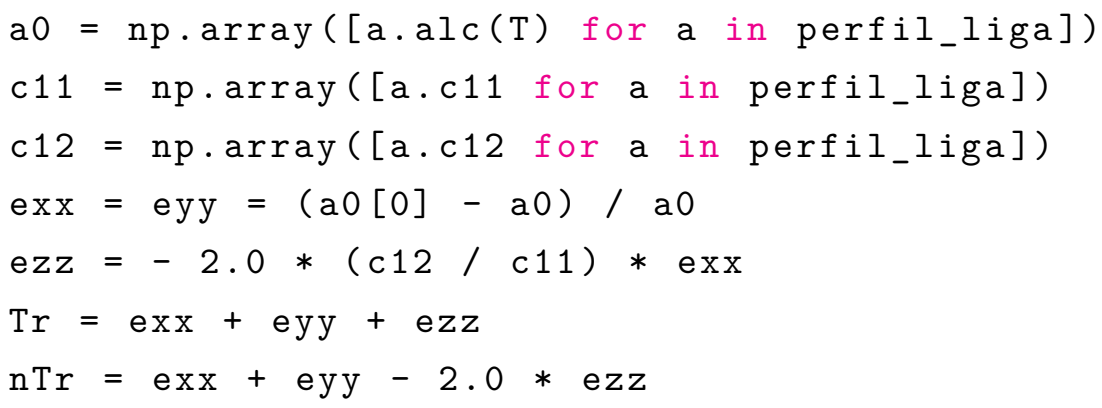

ajuste_c $=$ ac $*$ Tr 
110 \# Valor da PL supondo poço quântico simples.

111 pl_numerica_simples $=\{\}$

112 for mc in monocamadas:

ax.plot(monocamadas, pl_num, label=r"PL num \'\{e\}rica", marker='O')

ax.grid (True)

ax.set_xlabel ('Monocamadas')

ax.set_ylabel ('Evergia (eV)')

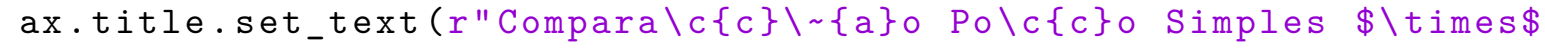
Experimento")

ax.set_xlim $([9,29])$

140 plt.plot()

141

142 \# \#\# PL com efeitos de tensão

143 pl_numerica_tensao $=\{\}$

144 for mc in monocamadas:

145 \# concentracao em cada camada do poco 
160

\# utilizando barreiras de 250 angstroms $\mathrm{x}_{-}$barreira $=[0.0] * \operatorname{int}(250.0 / \operatorname{InGaAs}(0.0) . \operatorname{alc}(\mathrm{T}))$

\# concentração

$\mathrm{x}_{-}$total $=\mathrm{x}_{-}$barreira $+\mathrm{x}_{-}$poco $+\mathrm{x}_{-}$barreira

\# considerando que o parametro de rede muda em cada camada tamanho_sistema $=n p \cdot \operatorname{sum}\left(\left[\operatorname{InGaAs}(\mathrm{x}) \cdot \operatorname{alc}(\mathrm{T})\right.\right.$ for $\mathrm{x}$ in $\mathrm{x}_{-}$total] $)$

\# interpola concentração ponto a ponto para $N$ pontos

$z_{-}$ang, $x_{-}$total = interpola_concentracao (x_total,

tamanho_sistema, N)

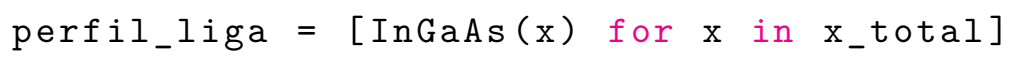


183

184

185

186

187

188

189

190

191

192

193

194

195

196

197

198

199

200

201

203

205

206

207

208

209

ax.set_xlim $([9,29])$

plt. legend(loc=' upper center', bbox_to_anchor=(0.50, -0.12), shadow $=$ True, $\operatorname{ncol}=2$ )

210 plt.savefig('figuras/ingaas_pl_77_comparacao.png', bbox_inches='tight') 



\section{ANEXO B - Bibliotecas}

Neste anexo apresentamos algumas bibliotecas que desenvolvemos para facilitar algumas operações realizadas no Anexo A.

\section{B.1 Constantes}

Estas são as constante físicas utilizadas em todos os procedimentos.

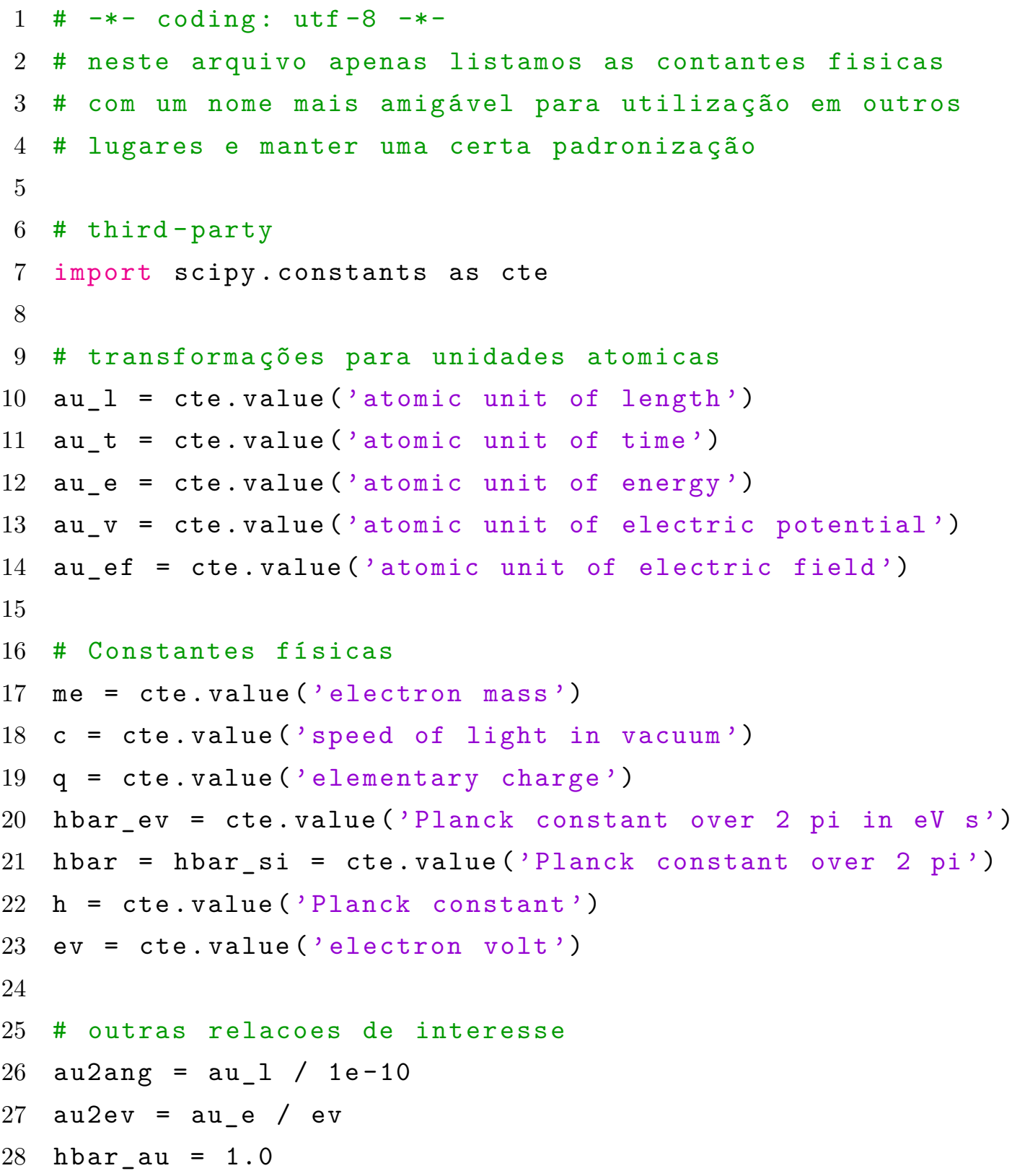


$\mathrm{me}_{-} \mathrm{au}=1.0$

\section{B.2 Database}

Parâmetros utilizados para calcular propriedades e perfis de potenciais de heteroestruturas semicondutoras.

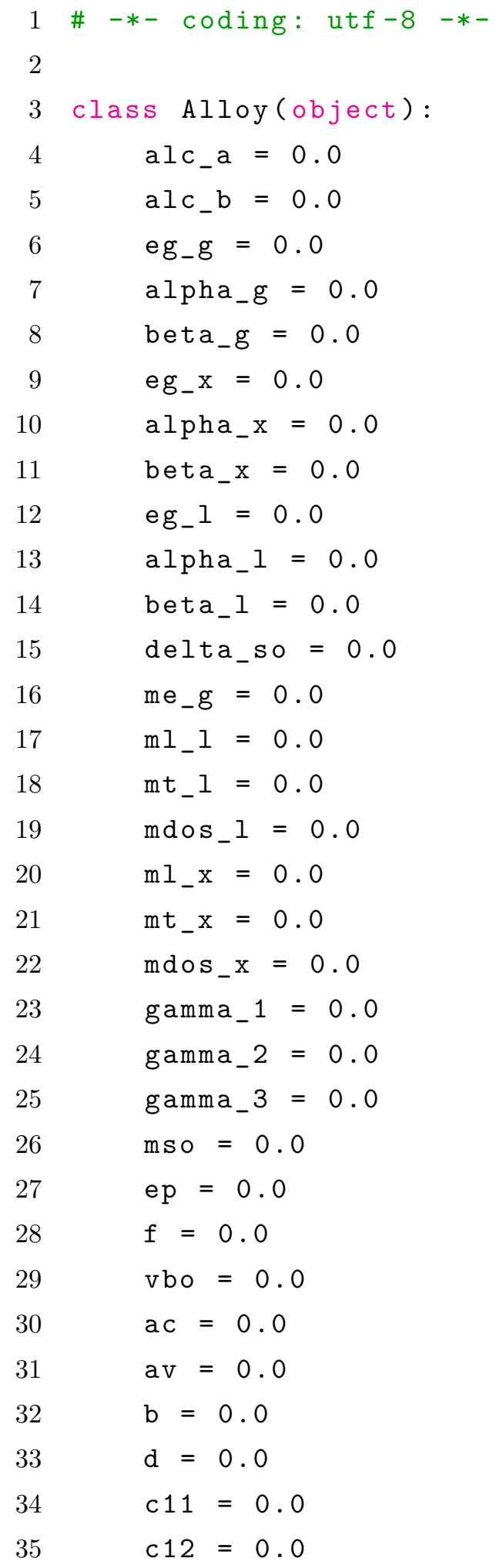


36

37

38

39

(1)

41

41

42

43

44

51

52

53

54

61

62

63

64

65

66

67

68

69

70

$$
c 44=0.0
$$

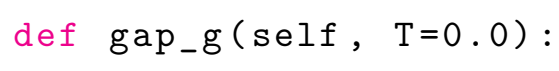


101

102

103

104

105

106

107

108

109

110

111

112

113 self.delta_so $=0.341$

self.me_g $=0.067$

self.ml_l $=1.9$

self.mt_l $=0.0754$

self.mdos_l $=0.56$

self.ml_ $x=1.3$

self.mt_x $=0.23$

self.mdos_ $x=0.85$

self.gamma_1 $=6.98$

self.gamma_ $2=2.06$

self.gamma_ $3=2.93$

self.mso $=0.172$

self.ep $=28.8$

self. $f=-1.94$

self.vbo $=-0.8$

self.ac $=-7.17$

self.av $=-1.16$

self.b $=-2.0$

self.d $=-4.8$

self.c11= 1221.0

self.c12 $=566.0$

self.c44 $=600.0$

$$
\text { def__init__(self): }
$$


118

119

120

121

122

123

124

125

126

127

128

129

130

131

132

133

134

135

136

137

138

139

140

141

142

143

144

145

146

147

148

149

150

151

152

153

154

155

156

157

158

159

$$
\begin{aligned}
& \text { self.ml_x }=0.97 \\
& \text { self.mt_x }=0.22 \\
& \text { self.mdos_x }=0.0 \\
& \text { self.gamma_1 }=3.76 \\
& \text { self.gamma_2 }=0.82 \\
& \text { self.gamma_3 }=1.42 \\
& \text { self.mso }=0.28 \\
& \text { self.ep }=21.1 \\
& \text { self.f }=-0.48 \\
& \text { self.vbo }=-1.33 \\
& \text { self.ac }=-5.64 \\
& \text { self.av }=-2.47 \\
& \text { self.b }=-2.3 \\
& \text { self.d }=-3.4 \\
& \text { self.c11 }=1250.0 \\
& \text { self.c12 }=534.0 \\
& \text { self.c44 }=542.0
\end{aligned}
$$

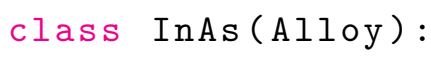


160

161

162

163

164

165

166

167

168

169

170

171

172

173

174

175

176

177

178

179

180

181

182

183

184

185

186

187

188

189

190

191

192

193

194

195

$$
\begin{aligned}
& \text { self.gamma_3 }=9.2 \\
& \text { self.mso }=0.14 \\
& \text { self.ep }=21.5 \\
& \text { self.f }=-2.9 \\
& \text { self.vbo }=-0.59 \\
& \text { self.ac }=-5.08 \\
& \text { self.av }=-1.0 \\
& \text { self.b }=-1.8 \\
& \text { self.d }=-3.6 \\
& \text { self.c11 }=832.9 \\
& \text { self.c12 }=452.6 \\
& \text { self.c44 } 4495.9
\end{aligned}
$$

class InGaAs (Alloy):

$$
\text { def _-init_- }(\operatorname{self}, x) \text { : }
$$

self.c_eg_g $=0.477$

self.c_eg_x $=1.4$

self.c_eg_l $=0.33$

self.c_delta_so $=0.15$

self.c_me_g $=0.0091$

self.c_mhh_001 = -0.145

self.c_mlh_001 = 0.0202

self.c_ep $=-1.48$

self.c_f $=1.77$

self.c_vbo $=-0.38$

self. $c_{-}$ac $=2.61$

self.alloy_1 = GaAs ()

self.alloy_2 = InAs ()

self.alc_a $=$ self.alloy_1.alc_a $*(1.0-x)+$

self.alloy_2.alc_a *

self.alc_b = self.alloy_1.alc_b*(1.0-x) +

self.alloy_2.alc_b*x

self.eg_g = self.alloy_1.eg_g*(1.0 - $\mathrm{x})+$

self.alloy_2.eg_g * $x-x *(1.0-x) *$ self.c_eg_g

self.alpha_g = self.alloy_1.alpha_g $*(1.0-x)+$

self.alloy_2.alpha_g *x

self.beta_g = self.alloy_1.beta_g * $(1.0-x)+$

self.alloy_2. beta_g * x

self.eg_x $=$ self.alloy_1.eg_x $*(1.0-x)+$

self.alloy_2.eg_x * $x_{-}-x *(1.0-x) *$ self.c_eg_ 
196

197

198

199

200

201

202

203

204

205

206

207

208

209

210

211

212

213

214

215

216

self.alpha_ $x=$ self.alloy_1.alpha_x $*(1.0-x)+$ self.alloy_2.alpha_x * x self.beta_ $x=\operatorname{self} . \mathrm{alloy}_{-} 1 . \mathrm{beta}_{-} \mathrm{x} *(1.0-\mathrm{x})+$ self.alloy_2. beta_x * self.eg_l = self.alloy_1.eg_l $1(1.0-x)+$ self.alloy_2.eg_l * $x_{-}-\mathrm{x} *(1.0-\mathrm{x}) *$ self.c_eg_l self.alpha_l = self.alloy_1.alpha_l 1 (1.0 - $x)+$ self.alloy_2.alpha_1 * self.beta_l = self.alloy_1.beta_l $1 *(1.0-x)+$ self.alloy_2.beta_l $* x$ self.delta_so = self.alloy_1.delta_so $*(1.0-\mathrm{x})+$ self.alloy_2.delta_so $* x$ self.me_g = self.alloy_1.me_g $*(1.0-x)+$

self.alloy_2.me_g * $x_{-}-\mathrm{x} *(1.0-\mathrm{x}) *$ self.c_me_g self.ml_l = self.alloy_1.ml_l $l_{-}(1.0-x)+$

self.alloy_2.ml_l * $\mathrm{x}$ self.mt_l = self.alloy_1.mt_l $1(1.0-\mathrm{x})+$

self.alloy_2.mt_l * $\mathrm{x}$ self.mdos $_{-} 1=\operatorname{self}_{\text {alloy }} 1 . \mathrm{mdos}_{-} 1 *(1.0-\mathrm{x})+$ self.alloy_2.mdos_l $* x$ self.ml_ $x=\operatorname{self} \cdot a l l o y_{-} 1_{1} m_{-} x *(1.0-x)+$ self.alloy_2.ml_x * $\mathrm{x}$ self.mt_ $x=$ self.alloy_1.mt_x $*(1.0-x)+$ self.alloy_2.mt_x * x self.mdos_x $=$ self.alloy_1.mdos_x $*(1.0-x)+$ self.alloy_2.mdos_x * x self.gamma_1 = self.alloy_1.gamma_1 $*(1.0-\mathrm{x})+$ self.alloy_2.gamma_1*x self.gamma_ $2=$ self.alloy_1.gamma_ $2 *(1.0-\mathrm{x})+$ self.alloy_2.gamma_2*x self.gamma_3 = self.alloy_1.gamma_3*(1.0- $x)+$ self.alloy_2.gamma_3*x self.mso $=$ self.alloy_1.mso $*(1.0-x)+$ self.alloy_2.mso * x self.ep $=$ self.alloy_1.ep $*\left(1.0-y_{-}\right)+$self.alloy_2.ep $^{2}$ $* \mathrm{x}-\mathrm{x} *(1.0-\mathrm{x}) *$ self.c_ep self.f = self.alloy_1.f*(1.0-x) + self.alloy_2.f*x - $\mathrm{X} *(1.0-\mathrm{x}) * \operatorname{self} . \mathrm{c}_{-} \mathrm{f}$ self. vbo = self.alloy_1. vbo $*(1.0-x)+$ self.alloy_2.vbo * $\mathrm{x}_{-} \mathrm{x} *(1.0-\mathrm{x}) *$ self.c_vbo self.ac $=$ self.alloy_1.ac $*(1.0-x)+$ self.alloy_2.ac $* \mathrm{x}-\mathrm{x} *(1.0-\mathrm{x}) * \operatorname{self} . \mathrm{c}_{-} \mathrm{ac}$ 
217 self.av $=$ self.alloy_1.av $*(1.0-x)+$ self.alloy_2.av

$* \mathrm{x}$

218

219

220

221

222

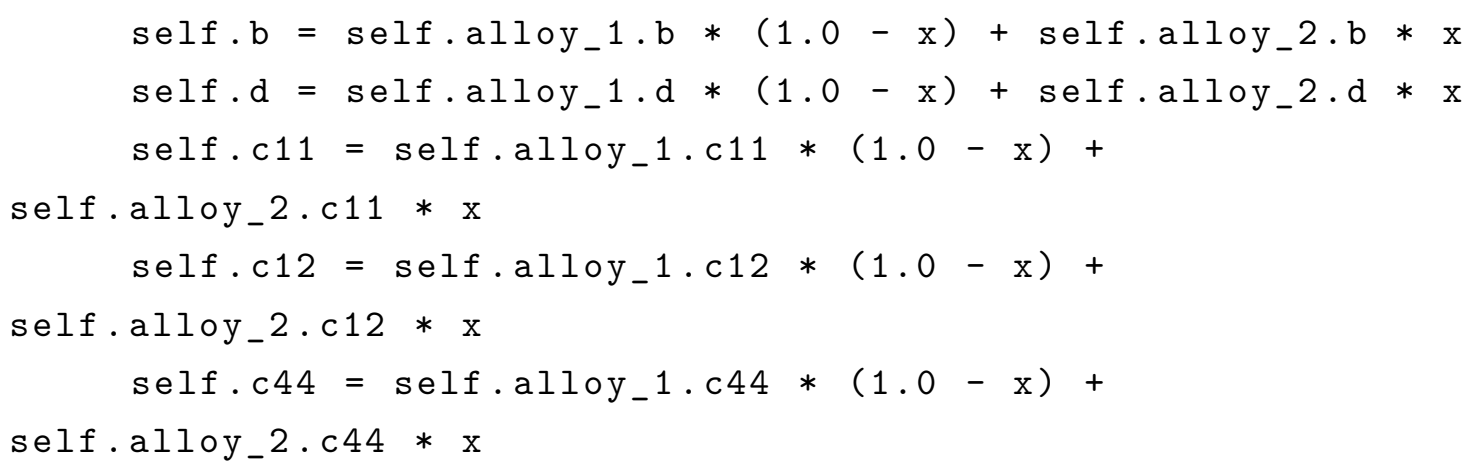

\section{B.3 Utilidades}

Funções diversas utilizadas para resolver o problema de autovalores e autovetore.

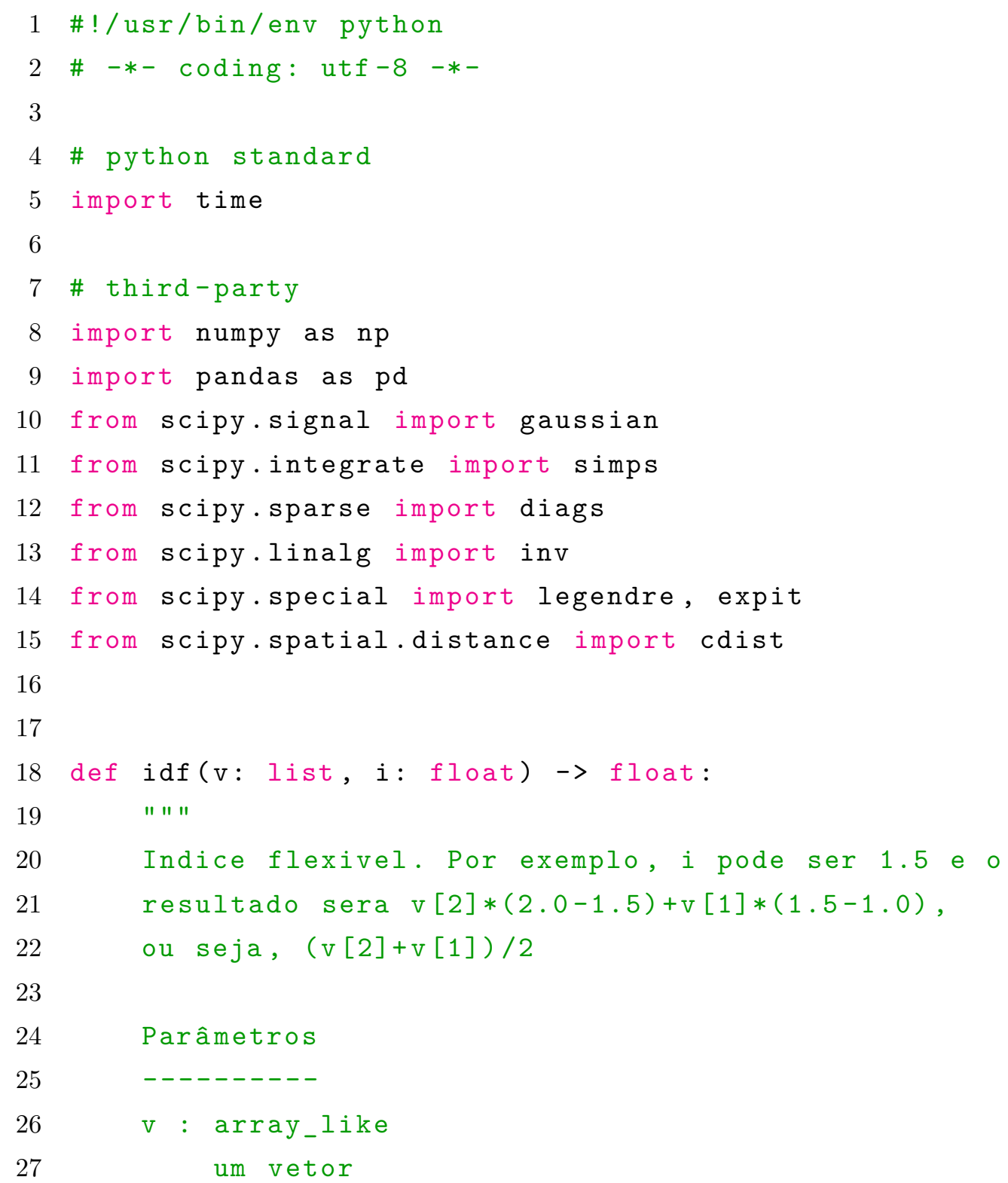


i : float um indice flexivel

\section{Retorno}

Uma interpolacao simples de v para o indice flexivel i " " "

$i_{-}$up $=\operatorname{int}(\mathrm{np} \cdot \operatorname{ceil}(\mathrm{i}))$

$i_{-}$down $=\operatorname{int}(\mathrm{np} \cdot f \operatorname{loor}(\mathrm{i}))$

if $i_{-}$down $<0.0$ :

return $\mathrm{v}[0]$

elif i_up >= $\operatorname{len}(\mathrm{v})-1$ :

return $\mathrm{v}[-1]$

$\mathrm{up}=i-$ float $\left(i_{\text {_down }}\right)$

down = float $\left(i_{-}\right.$up $)-i$

return $\mathrm{v}$ [i_up] * up + v[i_down $] *$ down

def dummy_comma(str_number:str)->str:

" " "

troca separador decimal '.' para ', '...

é preciso ser assim em alguns casos

Parâmetros

str_number : string

um string para troca '.' por ','

\section{Retorno}

uma string

" " "

c2at = str_number.replace (',', '@Q ')

$\mathrm{d} 2 \mathrm{c}=\mathrm{c} 2 \mathrm{at} \cdot \mathrm{replace}\left({ }^{\prime} \cdot{ }^{\prime},{ }^{\prime},{ }^{\prime}\right)$

return d2c.replace('@e', '.')

def as_si(x:float, ndp:int)->str:

" " "

coloca um número [x] em no si com ndp casas decimais

$$
\text { Parâmetros }
$$

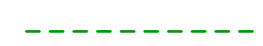


70

$\mathrm{x}$ : float

um numero real

ndp : int

o número de casas decimais

Retorno

O número $x$ formatado em notação cientídica com ndp casas " " "

$s=,\{x: 0 .\{n d p: d\} e\}, \cdot f o r m a t(x=x, n d p=n d p)$

$\mathrm{m}, \mathrm{e}=\mathrm{s} \cdot \mathrm{split}\left({ }^{\prime} \mathrm{e}\right.$ ')

return dummy_comma ( $r^{\prime}\{m: s\} \backslash t i m e s 0^{-}\{\{\{e: d\}\}\}^{\prime}$. format $(m=m$, $e=\operatorname{int}(\mathrm{e})))$

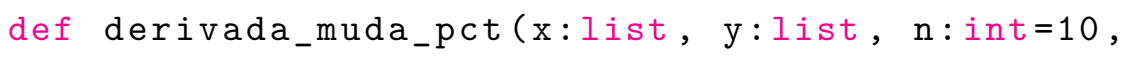
uma

certa porcentagem pela primeira vez, da esquerda para a direita

Parâmetros

$\mathrm{x}$ : array_like

um array com os valores em $x$

$\mathrm{y}$ : array_like

um array com os valores em y

$\mathrm{n}$ : int

numero do pontos para ignorar nas bordas

pct : float

a porcentagem da derivada que deve mudar

Retorno

0 indice do ponto $x$ onde dy/dx muda mais do que pct " " "

$\mathrm{der}_{-} \mathrm{y}=\mathrm{np} \cdot \operatorname{array}(\mathrm{y}[2:]-\mathrm{y}[:-2]) / \mathrm{np} \cdot \operatorname{array}(\mathrm{x}[2:]-\mathrm{x}[:-2])$

for $i$ in range $\left(n\right.$, len $\left.\left(\operatorname{der}_{-} y\right)\right)$ :

last_n $=n p \cdot \operatorname{average}\left(\operatorname{der} \_y[i-n: i-1]\right)$

if last_n $=0$ and der_y[i] $!=0 ।$ 
108

109

110

111

112

115

116

117

118

119

120

121

122

123

124

125

126

127

128

129

130

131

132

133

134

135

136

137

138

139

140

141

142

143

144

145

146

147

or last_n $!=0$ and np.abs (der_y[i]/last_n-1) >

pct :

def chutes_iniciais ( $n:$ int $=2$, tamanho: int $=1024$, $\mathrm{mu}:$ float $=$ None) $->$ list :

return int (len $(y) / 3)$

$$
\text { " " " }
$$

Retorna os $n$ primeiros polinomios de legendre modulados por uma gaussiana.

\section{Parâmetros}

$\mathrm{n}:$ int

o numero de vetores

tamanho : int

o tamanho dos vetores

$\mathrm{mu}$ : float

centro da gaussiana, entre 0 e 1

Retorno

$------$

Um array com $n$ arrays contendo os polinomios modulados " " " "

$\mathrm{sg}=\mathrm{np}$. linspace $(-1,1, \operatorname{tamanho})$

$\mathrm{g}=$ gaussian $($ tamanho, std=int $($ tamanho/100))

if $\mathrm{mu}$ :

$\mathrm{sigma}=\mathrm{np} \cdot \mathrm{ptp}(\mathrm{sg}) / 100$

$\mathrm{g}=(1.0 / \mathrm{np} \cdot \operatorname{sqrt}(2 * \mathrm{np} \cdot \mathrm{pi} * \operatorname{sigma} * * 2))$

$\mathrm{g} *=\mathrm{np} \cdot \exp (-(\mathrm{sg}-\mathrm{mu}) * * 2 /(2 * \operatorname{sigma} * * 2))$

$\mathrm{vls}=[\mathrm{g} *$ legendre $(i)(\mathrm{sg})$ for $i$ in range $(n)]$

return np.array(vls, dtype=np. complex_ $)$

def autovalor(z: list, V: list, psi: list, m: list) $->$ float: " " "

Calcula um autovalor como $E=\langle P s i|H| P s i\rangle /\langle P s i \mid P s i\rangle$ onde $\mathrm{H}=\mathrm{T}+\mathrm{V}, \mathrm{T}$ eh o operador de energia cinetica em uma dimensao

Parâmetros 
156

157

158

159

160 z : array_like

o eixo $z$

$\mathrm{V}$ : array_like

- potencial

psi : array_like

a funcao de onda psi(z)

$m$ : array_like

a massa efetiva $m *(z)$

Retorno

0 autovalor $\mathrm{E}=\langle\mathrm{Psi}|\mathrm{H}| \mathrm{Ps} i\rangle /\langle\mathrm{Ps} \mid \mathrm{Ps} i\rangle$

" " "

$\mathrm{N}=\operatorname{len}(\mathbf{z})$

$\mathrm{dz}=\mathrm{np} \cdot \operatorname{append}(\mathrm{z}[1:]-\mathrm{z}[:-1], \quad \mathrm{z}[1]-\mathrm{z}[0])$

$\mathrm{dz} 2=\mathrm{dz} * * 2$

$\mathrm{h}_{-} \mathrm{psi}=\mathrm{np} \cdot \operatorname{zeros}\left(\mathrm{N}, \operatorname{dtype}=\mathrm{np} \cdot \operatorname{complex}_{-}\right)$

for $i$ in range $(N)$ :

$\mathrm{h}_{-} \operatorname{psi}[\mathrm{i}]=((0.5 / \mathrm{dz} 2[\mathrm{i}]) *(1.0 / \mathrm{idf}(\mathrm{m}, \mathrm{i}+0.5)+$

$1.0 / \operatorname{idf}(\mathrm{m}, \quad i-0.5))+V[i]) * \operatorname{psi}[i]$

if $i>0$ :

$\mathrm{h}_{-} \operatorname{psi}[\mathrm{i}]+=-(0.5 / \mathrm{dz} 2[\mathrm{i}]) *(\mathrm{psi}[\mathrm{i}-1] / \mathrm{idf}(\mathrm{m}, \mathrm{i}-0.5))$

if $i<N-1$ :

$h_{-} \operatorname{psi}[i]+=-(0.5 / \mathrm{dz} 2[i]) *(\operatorname{psi}[i+1] / i d f(m, i+0.5))$

psi_h_psi $=\operatorname{simps}\left(p s i \cdot \operatorname{conj}() * h_{-} p s i, z\right)$

return (psi_h_psi / simps(psi.conj()*psi, z)).real

def normaliza(z:list, psi:list)->tuple:

" " "

normaliza uma função de onda psi para um espaço z

Parâmetros

z : array_like

o eixo $z$

psi : array_like

Retorno

Psi / $\operatorname{sqrt}(<\mathrm{Psi}|\mathrm{Psi}\rangle)$, sqrt (<Psi|Psi $>$ )

" " " 
190

191

192

193

194

195

196

197

198

199

200

201

202

203

204

205

206

207

208

209

210

211

212

213

214

215

216

217

218

219

220

221

222

223

224

225

226

227

228

229

230

$z=n p \cdot \operatorname{array}(z)$

psi $=n p \cdot \operatorname{array}\left(p s i\right.$, dtype=np.complex $\left.{ }_{-}\right)$

psi2 $=$ psi $*$ psi. $\operatorname{conj}()$

$A=n p \cdot \operatorname{sqrt}(\operatorname{simps}(\operatorname{psi2}, z))$

return psi / A, A

def interacao_inversa( $z$ : list, V: list, $m$ : list, $n \max :$ int $=20$, autovalores: list $=[]$, remover_repetidos:bool=True) ->dict:

" " "

Calcula os autovetores e autovalores para uma partícula de massa efetiva $m$, submetida a um potencial $V$ no espaço $z$ Todas as entradas e saidas devem ser em unidades atômicas

Parâmetros

z : array_like

a malha espacial

$\mathrm{V}$ : array_like

- potencial em cada ponto da malha espacial

$\mathrm{m}$ : array_like

a massa efetiva da partícula em cada ponto da malha espacial

$n \max$ : int

- número de chutes iniciais a serem utilizados entre $\min (\mathrm{V})$ e $\max (\mathrm{V})$

autovalores : array_like

mesmo que aproximados, ajudam o algoritmo a convergir

com maior velocidade e precisão

remover_repetidos : bool

remove os autoestados repetidos ou considerados "ruins"

Retorno

Um dicionário com as seguintes chaves: \{

"autovalores": [um array com autovalores],

"autovetores": [um array de arrays com autovetores],

"contadores": [array com \# iterações por autoestado],

"precisoes": [array com precisão de cada autovalor],

"cronometros": [array com tempo para calcular cada

autovetor], 
"euclides2": [array com distância euclidiana quadrada]* \}

* A distância euclidiana no caso indica a convergência do autovetor com ele mesmo, sendo a soma dos quadrados das diferenças ponto a ponto de um autovetor em um passo n e um passo n-1

" " "

\# converte para array de numpy para facilitar

$\mathrm{z}=\mathrm{np} \cdot \operatorname{array}(\mathrm{z})$

$\mathrm{V}=\mathrm{np} \cdot \operatorname{array}(\mathrm{V})$

$\mathrm{m}=\mathrm{np} \cdot \operatorname{array}(\mathrm{m})$

243

$\mathrm{N}=\mathrm{z} \cdot \mathrm{size}$

4

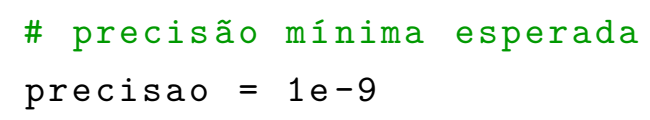

261 
278

279

280

281

282

283

285

286

287

288

289

290

291

292

293

294

295

296

297

298

299

300

301

302

303

304

305

306

307

308

euclides $2=n p \cdot z \operatorname{eros}(n \max )$

for $s$ in range (nmax):

last_ev $=1.0 \quad \#$ autovalor inicial fake

last_es $=n p \cdot z \operatorname{zeros}\left(N, \mathrm{dtype}_{\text {lat }} \cdot \mathrm{complex}_{-}\right)$\# autovetor inicial

shift $=$ previsao[s]

\# Desloca o potencial do Hamiltoniano por shift

sub_diag $=n p \cdot \operatorname{zeros}\left(N_{-1}\right.$, dtype=np.complex_ $)$

main_diag $=n p \cdot \operatorname{zeros}(N$, dtype=np.complex_ $)$

\# constroi as diagnais da matriz, a principal e as duas semi

\# principais

for $i$ in range(N):

try:

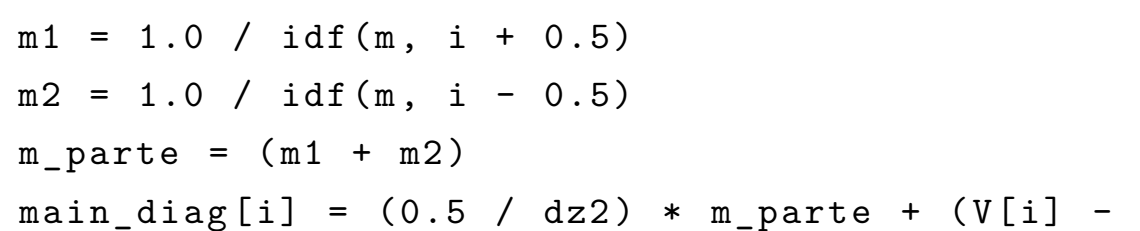

$\operatorname{shift)}$

except :

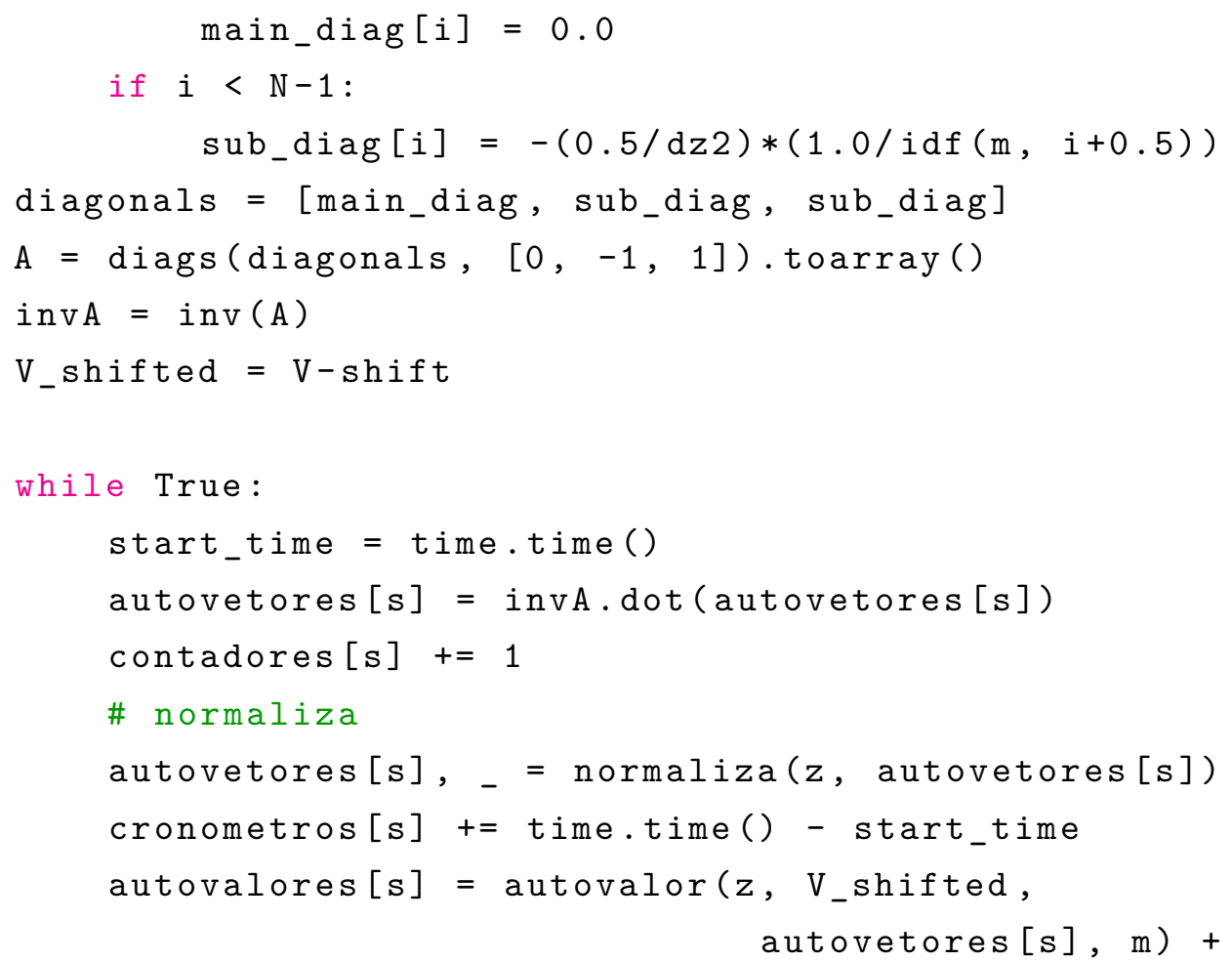

shift 
310

331

332

333

334

335

336

337

338

339

340

341

342

343

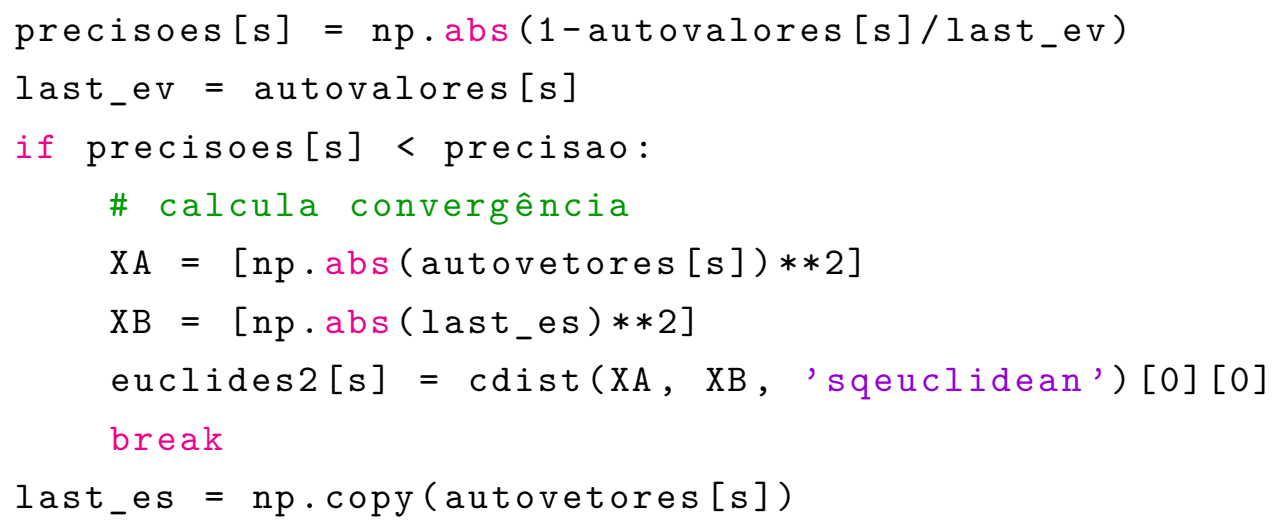

$0.1 \backslash$

7

or autovalores[i] >np.max (V):

continue

\# remove os estados nao confinados lateralmente state $=$ autovetores $[i] \cdot \operatorname{copy}()$

state_l = state $[:$ iz_left $]$

state_m = state[iz_left:iz_right]

state_r = state[iz_right:]

int_left $=\operatorname{simps}($ state_l $*$ state_l.conj(), ।

$$
\left.z\left[: i_{-} l e f t\right]\right) \text {.real }
$$

int_mid $=\operatorname{simps}($ state_m*state_m. $\operatorname{conj}()$,

$$
z\left[i z_{-}\right. \text {left:iz_right]).real }
$$

int_right $=\operatorname{simps}($ state_r $*$ state_r.conj ()$, ।$

$$
\left.z\left[i z_{-} r i g h t:\right]\right) \text {.real }
$$

if int_left+int_right > int_mid:

continue 
351

356

358

golden_ones.append (i)

else:

golden_ones $=[i$ for $i$ in range(len(autovalores)) $]$

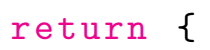

\section{B.4 Onda Plana}

Bibliotecas relacionadas a evolução temporal de onda plana, numericamente e analiticamente.

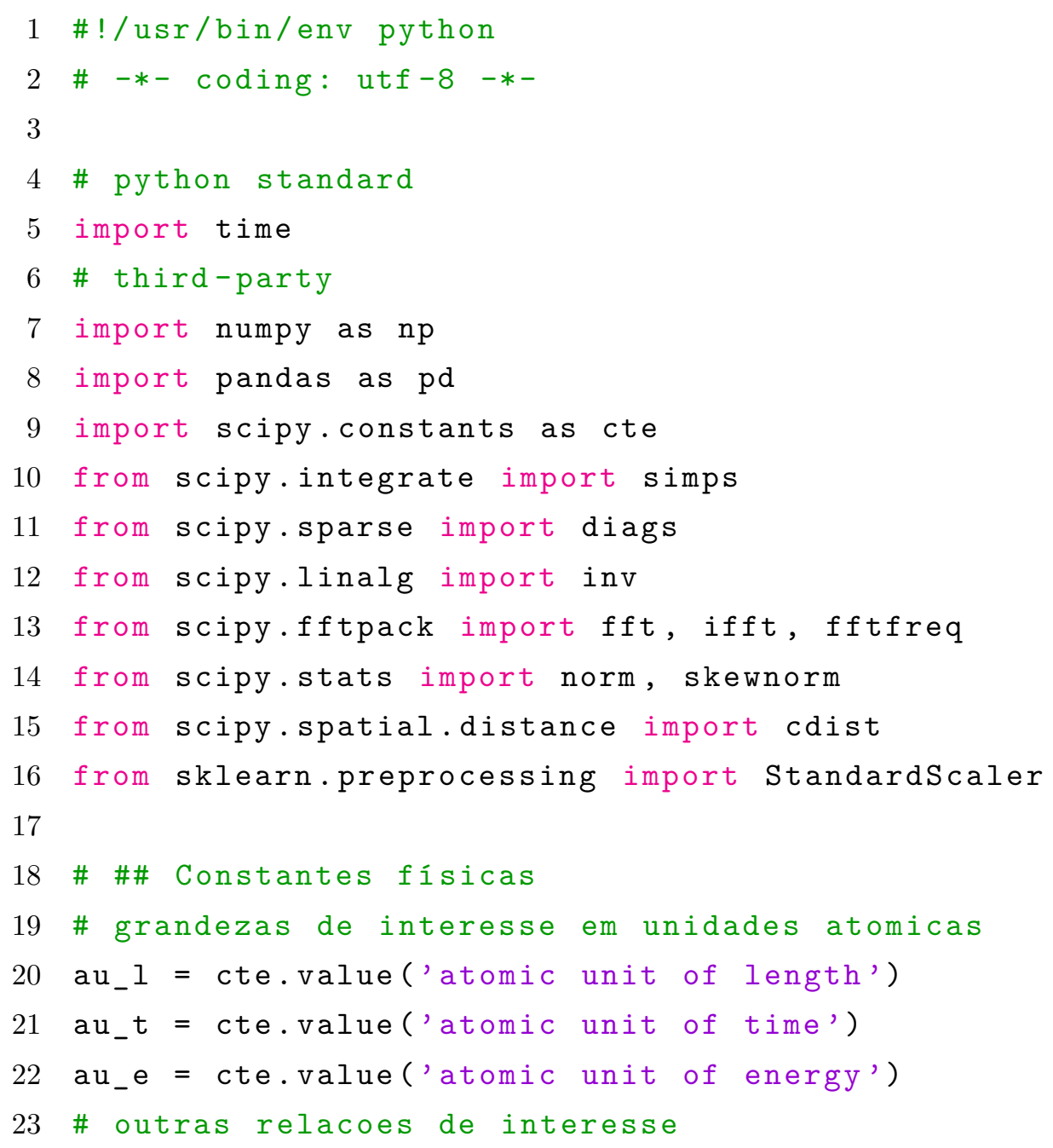


ev $=$ cte.value ('electron volt')

$\mathrm{au} 2 \mathrm{ang}=\mathrm{au} u_{-} 1 / 1 \mathrm{e}-10$

$\mathrm{au} 2 \mathrm{ev}=\mathrm{au} \mathrm{u}_{-} \mathrm{e} / \mathrm{ev}$

def evolucao_analitica $(z i=-20.0, z f=20, E=150.0$, deltaz=5.0, $\mathrm{L}=100.0, \mathrm{~N}=1024$, tempo=2.1739442773545673e-14, simples=True ) :

" " "

Evolui uma onda com energia [E] e desvio padrão [deltaz] da posição inicial [zi] até a posição final [zf]. A evolução ocorre em um espaço unidimensional de lamanho [L] partido em [N] pontos. Esta evolução é pseudo-analítica, pois assume que a integração numérica pode ser realizada com grande precisão.

Parâmetros

zi : float

Posição inicial em Angstrom

zf : float

Posição final em Angstrom

$E$ : float

Energia da onda em eV

deltaz : float

Desvio padrão em Angstrom

L : float

Compimento do sistema em Angstrom

$\mathrm{N}$ : int

Número de pontos em que [L] será partido tempo: float

0 tempo para evolução em segundos simples : bool

Se True, evolui pelo tempo t, se False, dá preferência para atingir $\mathrm{zf}$

Retorna

resumo : dict

Um dicionário com as seguintes chaves:

- [z_si] malha de pontos em z (SI)

- [z_au] malha de pontos em z (AU) 


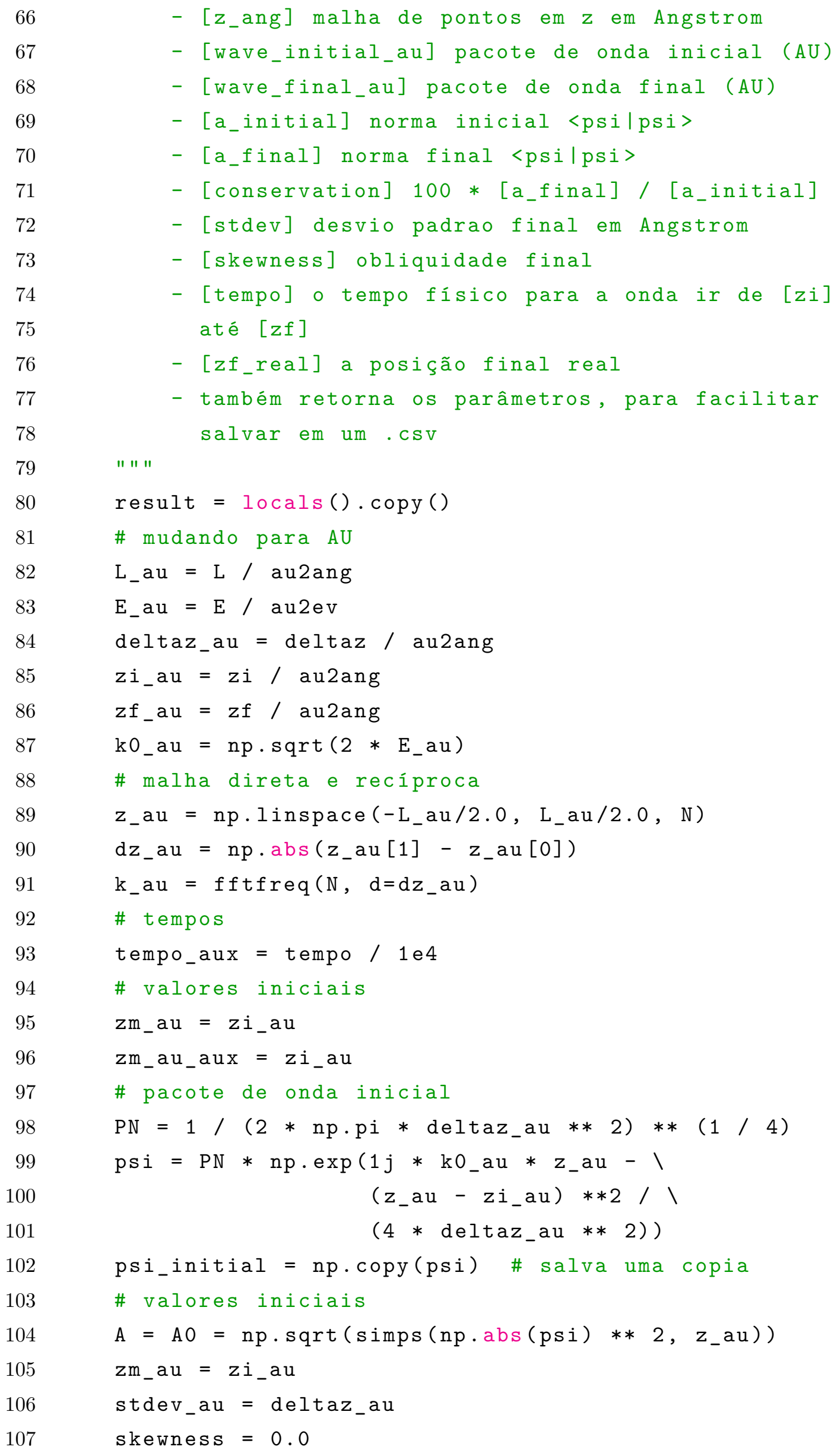


108

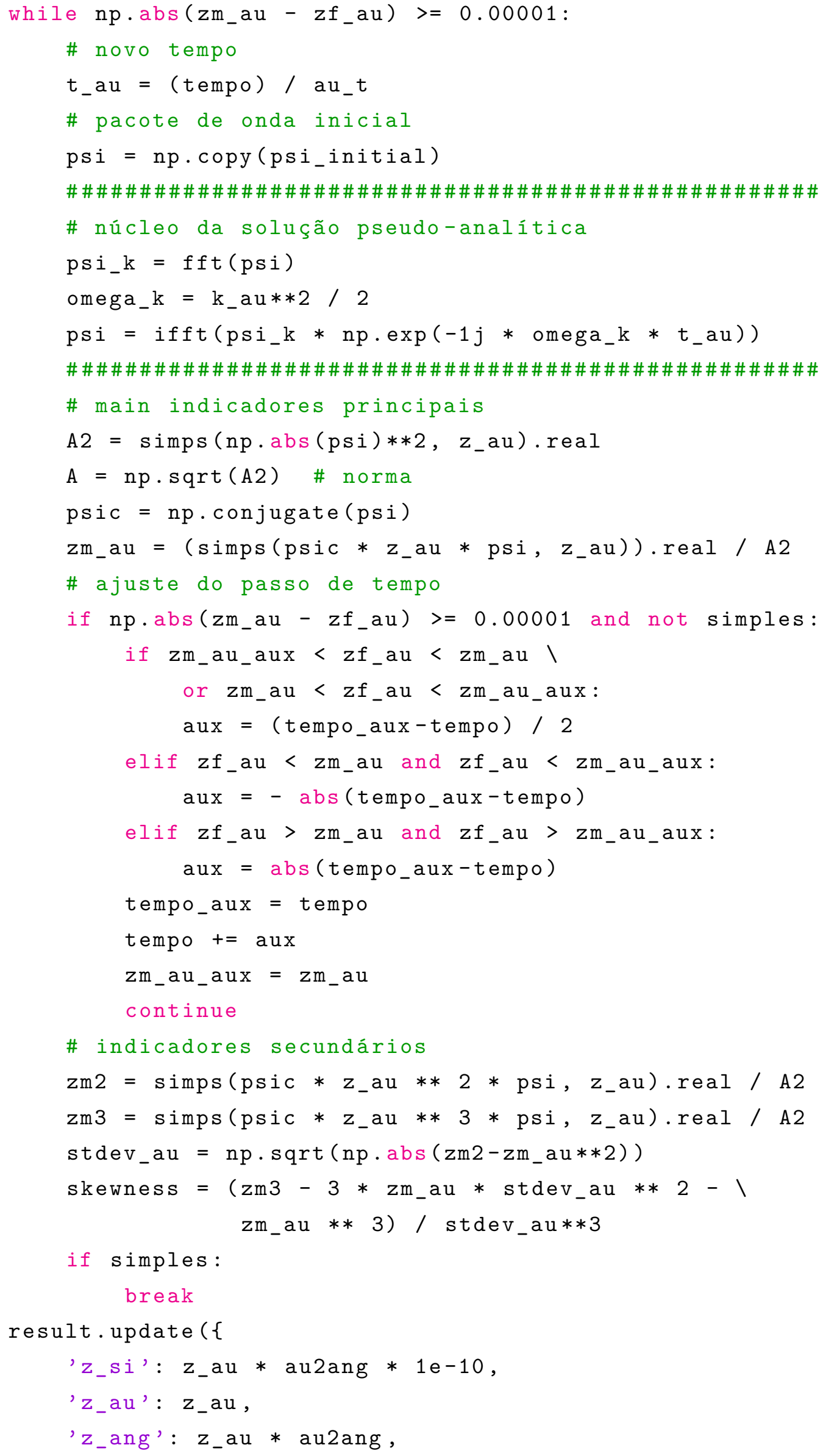


150

151

152

153

154

155

156

157

158

159

160

161

162

163

164

165

166

167

168

169

170

171

173

174

175

176

177

178

179

180

181

182

183

184

185

186

187

188

189

190

191 'wave_initial': psi_initial,

'wave_final': psi ,

'a_initial': AO,

'a_final': A,

'conservation': $100 * \mathrm{~A} / \mathrm{AO}$,

'stdev': stdev_au * au2ang,

'skewness': skewness ,

'tempo': tempo,

'zf_real': zm_au * au 2 ang

\})

return result

def evolucao_numerica $(z i=-20.0, \quad z f=20, E=150.0$, deltaz=5.0, $\mathrm{L}=100.0, \mathrm{~N}=1024, \mathrm{dt}=1 \mathrm{e}-20$, method=' $\mathrm{pe}$ ', tempo=2.1739442773545673e-14, simples $=$ False ) :

" " "

Evolui uma onda com energia [E] e desvio padrão [deltaz] da posição inicial [zi] até a posição final [zf]. A evolução ocorre em um espaço unidimensional de lamanho [L] partido em [N] pontos. 0 método a ser utilizado deve ser escolhido entre Pseudo-Espectral, Crank-Nicolson e Runge-Kutta

Parâmetros

zi : float

Posição inicial em Angstrom

zf : float

Posição final em Angstrom

$E$ : float

Energia da onda em eV

deltaz : float

Desvio padrão em Angstrom

L : float

Compimento do sistema em Angstrom

$\mathrm{N}$ : integer

Número de pontos em que [L] será partido

dt : float

0 passo de tempo em segundos

method : string 
192

193

194

195

196

197

198

199

200

201

202

203

204

205

206

207

208

209

210

211

212

213

214

215

216

217

218

219

220

221

222

223

224

225

226

227

228

229

230

231

232

233

são aceitos:

- 'pe': Pseudo-Espectral

- 'cn' : Crank-Nicolson

- 'rk' : Runge-Kutta

tempo : float

0 tempo para evolução em segundos

simples : bool

Se True, evolui pelo tempo t, se False, dá

preferência para atingir zf

\section{Retorna}

resumo : dict

Um dicionário com as seguintes chaves:

- [z_si] malha de pontos em z (SI)

- [z_au] malha de pontos em z (AU)

- [z_ang] malha de pontos em z em Angstrom

- [wave_initial_au] pacote de onda inicial (AU)

- [wave_final_au] pacote de onda final (AU)

- [a_initial] norma inicial <psilpsi>

- [a_final] norma final <psilpsi>

- [conservation] $100 *$ [a_final] / [a_initial]

- [stdev] desvio padrao final em Angstrom

- [skewness] obliquidade final

- [zf_real] a posição final real

- [program_time] o tempo de processamento

- [iterations] o número de iterações de tempo [dt]

- [tempo] é o tempo do sistema em segundos

- também retorna os parâmetros, para facilitar salvar em um. csv

" " "

result $=$ locals ()$\cdot \operatorname{copy}()$

\# mudando para AU

$L_{-} a u=L /$ au 2 ang

$d t_{-} a u=d t / a u_{-} t$

$E_{-} \mathrm{au}=\mathrm{E} / \mathrm{au} 2 \mathrm{ev}$

deltaz_au = deltaz / au2ang

$z i_{-} a u=z i /$ au2ang

$z f_{-} a u=z f /$ au2ang

$\mathrm{k} 0_{-} \mathrm{au}=\mathrm{np} \cdot \operatorname{sqrt}\left(2 * \mathrm{E}_{-} \mathrm{au}\right)$

\# malha direta e recíproca

$z_{-} a u=n p . l i n s p a c e\left(-L_{-} a u / 2.0, L_{-} a u / 2.0, N\right)$ 
$d z_{-} a u=n p \cdot a b s\left(z_{-} a u[1]-z_{-} a u[0]\right)$

$k_{-} a u=f f t f r e q\left(N, d=d z_{-} a u\right)$

\# começa a contar o tempo do programa

time_inicial = time.time ()

\# runge-kutta 4 th order

if $\operatorname{method}==$ ' $r k$ ':

$$
\mathrm{alpha}=1 \mathrm{j} /\left(2 * \mathrm{~d} z_{-} \mathrm{au} * * 2\right)
$$




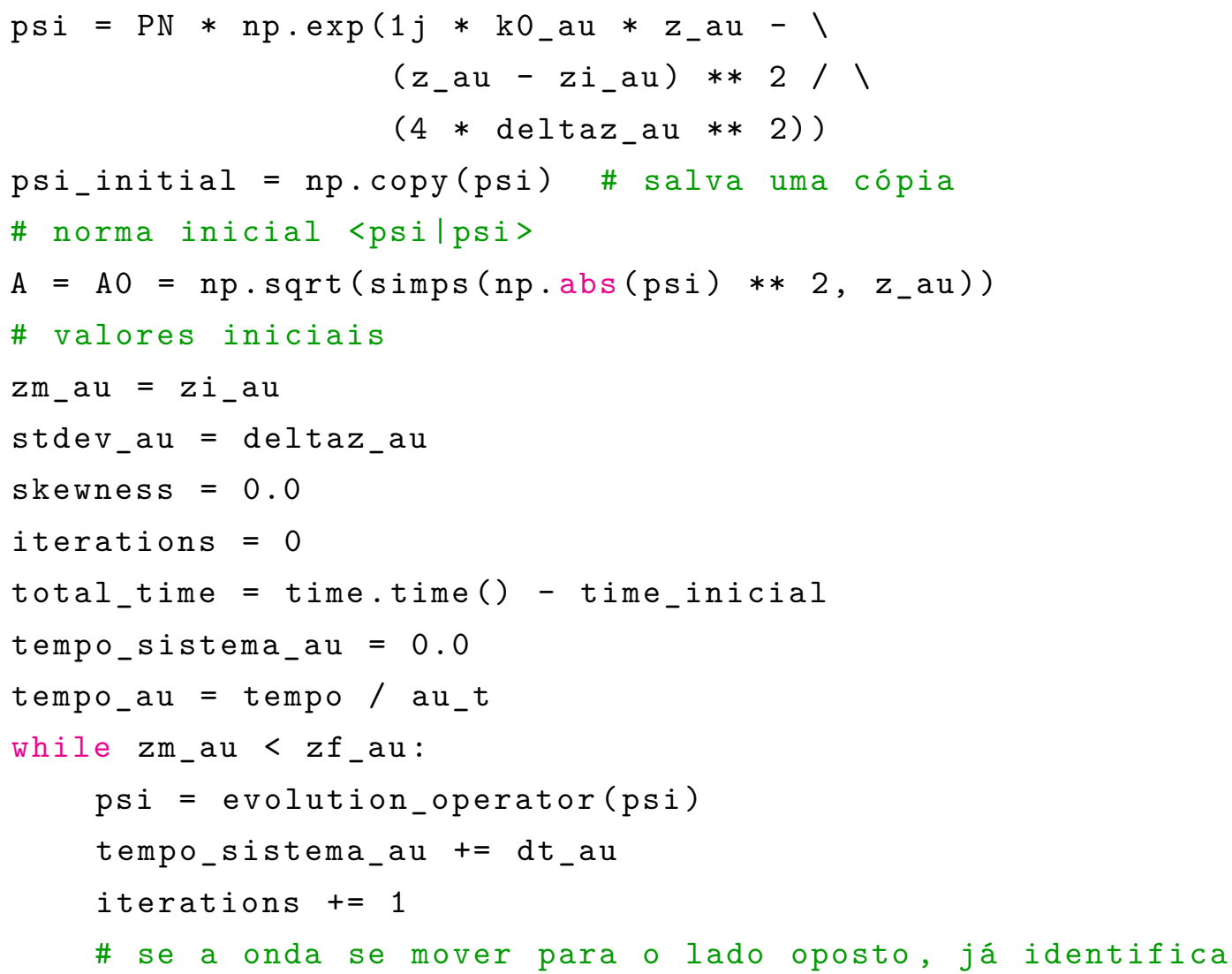




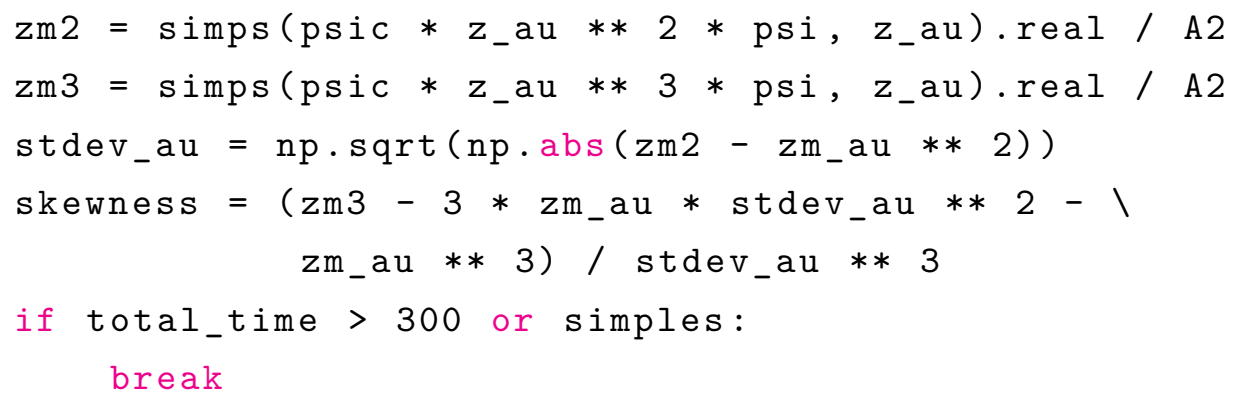

\section{B.5 Oscilador Harmônico Quântico}

Funções para resolver o problema do oscilador harmônico quântico, analiticamente e numericamente.

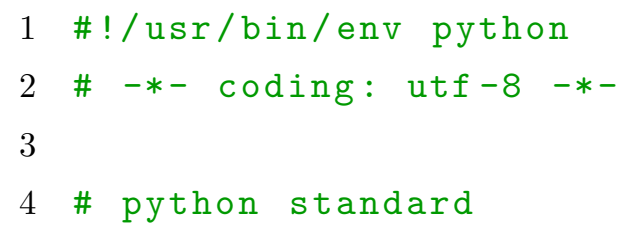




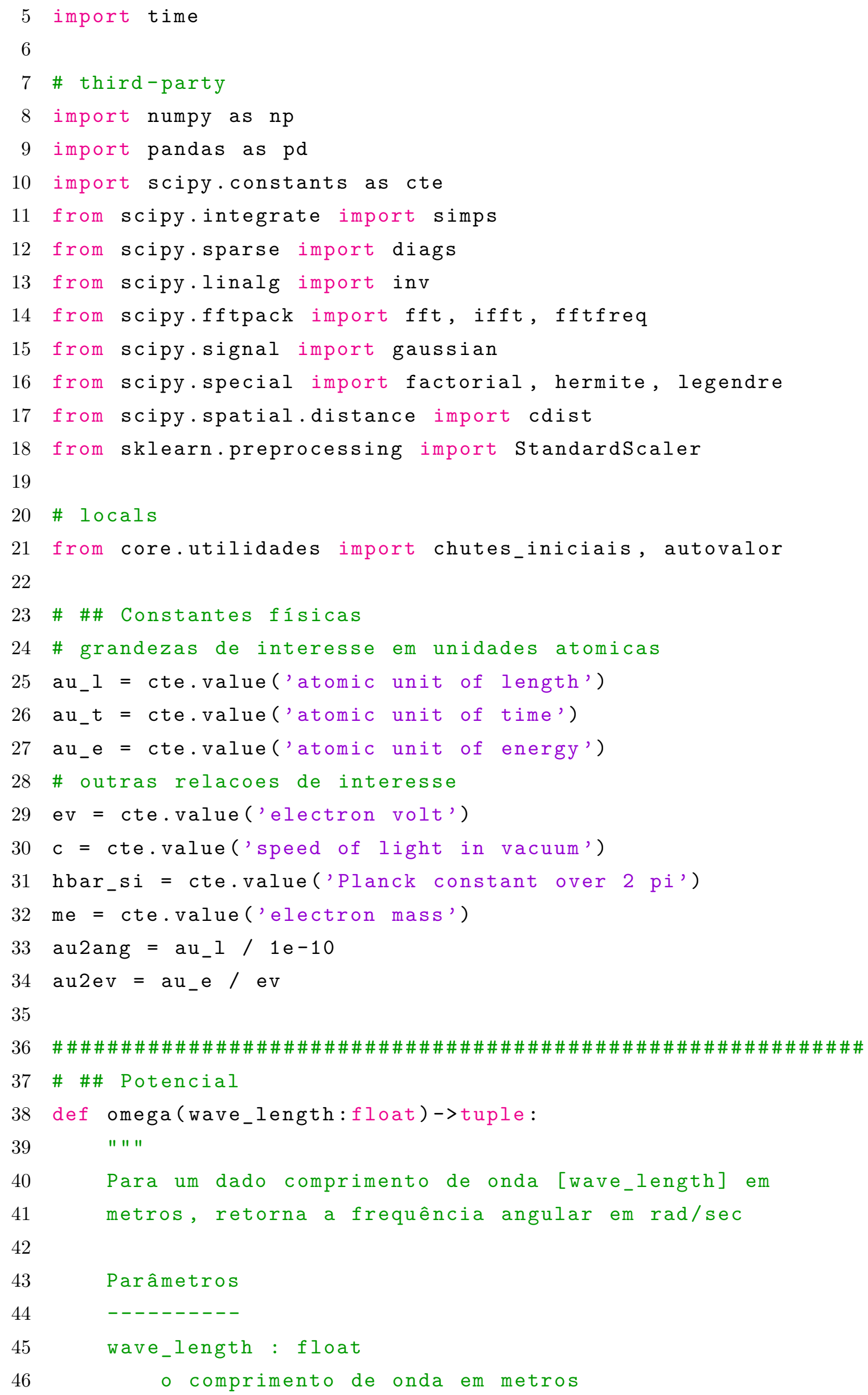


47

48

49

50

51

56

Retorna

ang_freq : tuple frequência angular ( ang_freq_si, ang_freq_au) 
89

90

91

96

96

97

98

99

100

101

102

103

104

105

106

107

108

109

110

111

112

113

114

115

116

117

118

119

120

121

122

123

124

125

126

127

128

129

return $z_{-} s i, z_{-} a u, v_{-} e v, v_{-} s i, v_{-} a u$

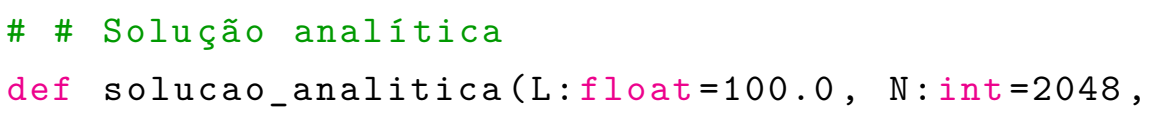




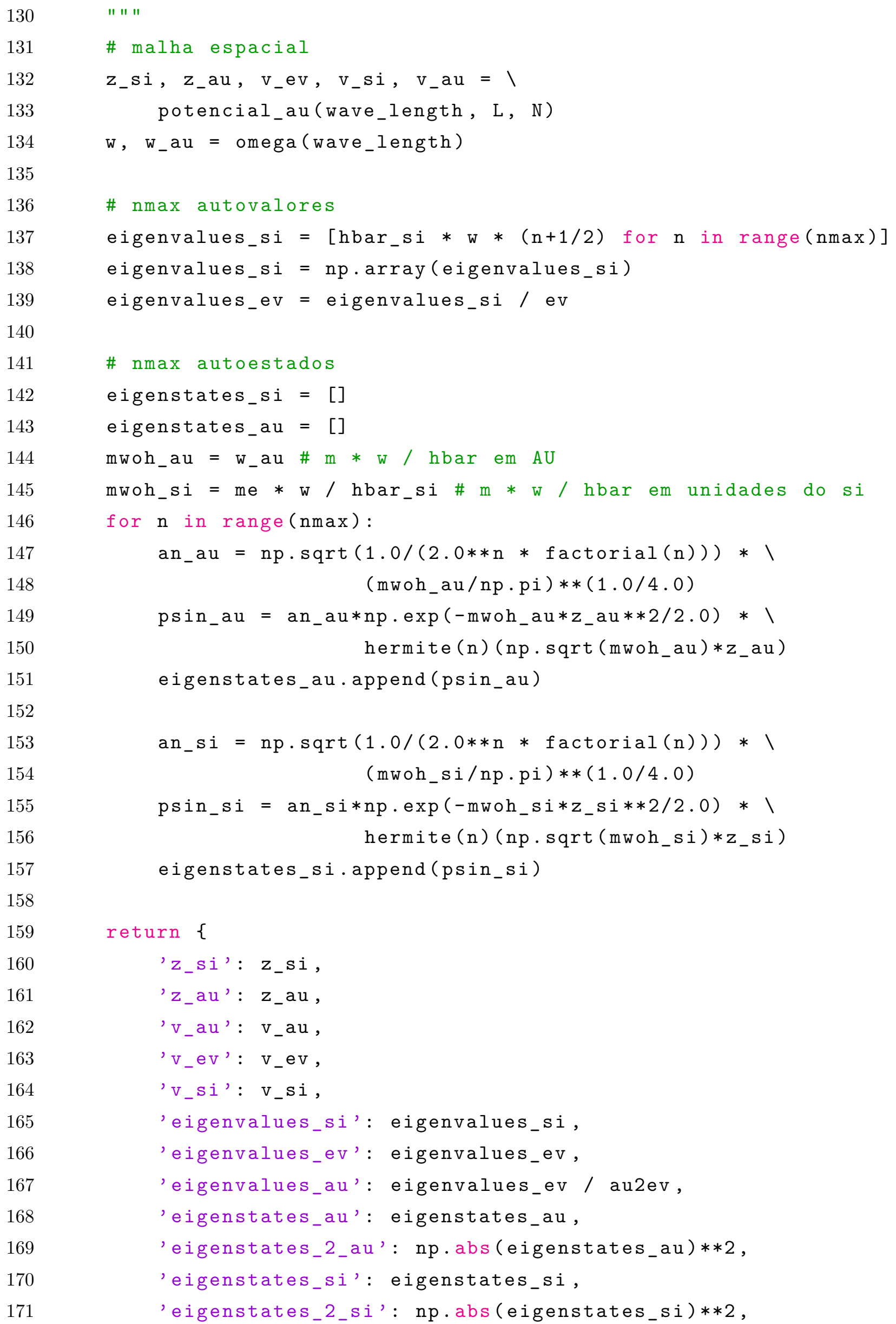


172

173

174

177

178

179

180

181

182

183

184

185

186

187

188

189

190

191

192

193

194

195

196

197

198

199

200

201

202

203

\}

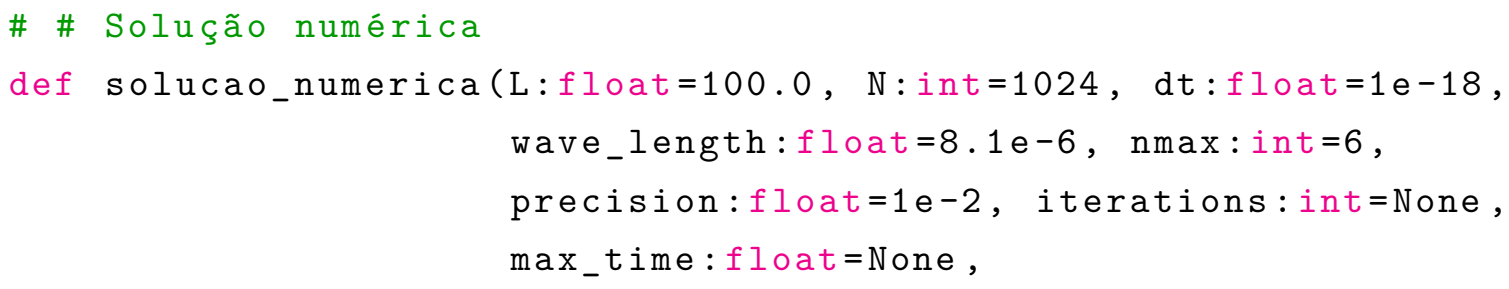


213

214

215

216

217

218

219

220

221

222

223

224

225

226

227

228

229

230

231

232

233

234

235

236

237

238

239

240

241

242

243

244

245

246

247

248

249

250

251

252

253

254
Returns

result : dictionary

Um dicionário com as seguintes chaves:

- 'z_si' malha espacial (SI)

- 'z_au' malha espacial (AU)

- 'v_au' potencial (AU)

- 'v_ev' potencial (eV)

- 'V_si' potencial (SI)

- 'eigenvalues_si' autovalores (Joules)

- 'eigenvalues_ev' autovalores (eV)

- 'eigenvalues_au' autovalores (AU)

- 'eigenstates_au' autofunções (AU)

- 'eigenstates_2_au' autofunções na forma $\mid$ psil`2 (AU)

- 'eigenstates_si' autofunções (SI)

- 'eigenstates_2_si' autofunções na forma $\mid$ psil|-2 (SI)

- 'iterations" um array com o número de iterações por autovalor

- 'timers' um array com o tempo de processamento por autovalor

- 'precisions' um array com a precisão por autovalor

- 'chebyshev' distânca de chebyshev por autofunção

- 'seuclidean' distânca euclidiana por autofunção

- 'sqeuclidean' distânca quadrada euclidiana por aut of unção

" " "

\# soluções analiticas

analytical = solucao_analitica $(\mathrm{L}=\mathrm{L}, \mathrm{N}=\mathrm{N}$, wave_length=wave_length, $\mathrm{nmax}=\mathrm{nmax}$ )

eigenvalues_ev_ana = analytical ['eigenvalues_ev']

eigenstates_au_ana = analytical ['eigenstates_au']

\# grid values

$z_{-} s i, z_{-} a u, v_{-} e v, v_{-} s i, v_{-} a u=1$

potencial_au (wave_length, L, N)

$d t_{-} a u=-1 j * d t / a u_{-} t$

precision /= 100 \# it is a percentage

forecast $=$ eigenvalues_ev_ana.copy () / au2ev

$\mathrm{dz2}=\left(z_{-} \mathrm{au}[1]-z_{-} \mathrm{au}[0]\right) * * 2$ 
\# split step

meff $=n p$.ones $(N)$

$\mathrm{d} z_{-} \mathrm{au}=\mathrm{np} \cdot \mathrm{abs}\left(z_{-} \mathrm{au}[1]-z_{-} \mathrm{au}[0]\right)$

$k_{-} a u=f f t f r e q\left(N, d=d z_{-} a u\right)$

exp_v2 $=n p \cdot \exp \left(-0.5 j * v_{-} a u * d t_{-} a u\right)$

$\exp p_{-}=n p \cdot \exp \left(-0.5 j *\left(2 * n p \cdot p i * k_{-} a u\right) * * 2 * d t_{-} a u\right)$

evolution_operator = lambda p:

exp_v $2 *$ ifft $\left(\exp \_t * f f t\left(e p_{-} v 2 * p\right)\right)$

\# chutes iniciais

if not eigenstates_au:

eigenstates_au = chutes_iniciais $(n=n \max$, tamanho=N)

eigenvalues_ev $=n p \cdot \operatorname{zeros}(n \max )$

counters $=n p \cdot z e r o s(n \max )$

timers $=n p \cdot z e r o s(n \max )$

precisions $=n p \cdot z e r o s(n \max )$

vectors_chebyshev $=n p \cdot z e r o s(n \max )$

vectors_sqeuclidean $=n p \cdot z e r o s(n \max )$

vectors_seuclidean $=n p \cdot z e r o s(n \max )$

\# matrix diagonals

sub_diag $=-(0.5 / \mathrm{dz2}) * \mathrm{np}$. ones $\left.(\mathrm{N}-1, \text { dtype=np.complex })_{-}\right)$

main_diag $=n p \cdot z \operatorname{eros}(N$, dtype=np.complex_ $)$

def get_inva( $v_{-}$shift=0.0):

"aplica um deslocamento no potencial, o mesmo que $\mathrm{H}^{\prime}=\mathrm{H}-$ shift "

main_diag $=\left(v_{-} a u-v_{-}\right.$shift $\left.+1.0 / d z 2\right)$

diagonals $=$ [main_diag, sub_diag, sub_diag]

return inv(diags (diagonals, $[0,-1,1]$ ). toarray ())

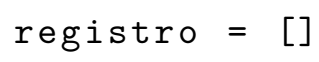


\# normalize

$\left.\left.z_{-} a u\right)\right)$

$\mathrm{A}=\mathrm{np} \cdot \operatorname{sqrt}(\operatorname{simps}(\mathrm{np} \cdot \mathrm{abs}($ eigenstates_au $[\mathrm{s}]) * * 2$,
295

296

297

298

299

300

301

302

303

304

305

306

307

308

309

310

311

312

313

314

315

316

317

318

319

320

321 if salvar and (counters[s] >= salvando $\backslash$

or (iterations and counters $\mathbf{s}]>=$ iterations)):

eigenstates_au[s] /=A
timers[s] += time.time ()$-$ start_time $^{-}$

psi2 $=(n p \cdot a b s($ eigenstates_au $[s]) * * 2) \cdot$ real

$\mathrm{av}=$ autovalor $\left(z_{-} \mathrm{au}, \mathrm{v}_{-} \mathrm{au}\right.$, eigenstates_au $[s]$, meff )

registro $+=[\{$

"nivel": $s$,

"iteracoes": min(salvando, iterations),

"autovalor": av,

"z": z,

"autovetor": p.real,

"autovetor2": p2

\} for $z, p, p 2$ in $z i p\left(z_{-} a u\right.$,

eigenstates_au[s], psi2)]

salvando $*=2$

if (iterations and counters [s] >= iterations) ।

or (max_time and timers[s] $>=\max _{-}$time) ।

or counters $[s] \% 1000==0$ :

ev = autovalor $\left(z_{-} a u, v_{-} a u\right.$,

eigenstates_au[s], meff)

eigenvalues_ev[s] $=$ ev $*$ au $2 \mathrm{ev} \# \mathrm{eV}$

precisions $[\mathrm{s}]=\mathrm{np} \cdot \mathrm{abs}(1-\mathrm{eigenvalues}$ _ev[s] /

1

eigenvalues_ev_ana [s])

if salvar:

filename $=$

'saidas/oscilador_harmonico_ \{\}$. p k l$ '

filename = filename.format (iterations)

pd.DataFrame (registro).to_pickle(filename)

iterations) ।

328 
329

330

331

332

333

334

335

336

337

338

339

340

341

342

343

344

345

346

347

348

349

350

351

352

353

$\left.\left.z_{-} a u\right)\right)$

354

355

356

357

358

359

360

$361 \#$

$362 \#$

$363 \#$

$364 \#$

$\mathrm{d} z_{-} \mathrm{au} * * 2$

$365 \quad \#$

$366 \quad \#$ or (not iterations and not max_time ।

and precisions [s] ( precision):

$X A=[$ eigenstates_au $[s]]$

$\mathrm{XB}=[$ eigenstates_au_ana $[\mathrm{s}]]$

vectors_chebyshev[s] = cdist $(\mathrm{XA}, \mathrm{XB}$,

'chebyshev') [0] [0]

vectors_seuclidean $[\mathrm{s}]=\operatorname{cdist}(\mathrm{XA}, \mathrm{XB}$,

'seuclidean') [0] [0]

vectors_sqeuclidean $[\mathrm{s}]=\operatorname{cdist}(\mathrm{XA}, \mathrm{XB}$,

'sqeuclidean') [0] [0]

break

elif method $==$ 'ii':

for $s$ in range(nmax):

last_ev $=1.0$

last_es $=n p \cdot \operatorname{zeros}\left(N\right.$, dtype $=n p \cdot c_{0}$ mplex $)$

shift $=$ forecast $[\mathrm{s}]$

invA $\quad=$ get_invA (shift)

$\mathrm{V}_{-}$shifted $=\mathrm{v}_{-}$au-shift

while True:

start_time = time.time ()

eigenstates_au[s] = invA.dot (eigenstates_au[s])

counters $[s]+=1$

\# normalize

$A=n p \cdot \operatorname{sqrt}(\operatorname{simps}$ (eigenstates_au[s] $* 1$

eigenstates_au[s]. $\operatorname{conj}()$,

eigenstates_au [s] /= A

timers $[s]+=$ time.time () - start_time

if (iterations and counters $[s]>=$ iterations) ।

or (max_time and timers[s] $>=\max _{-}$time) ।

or counters[s] $\% 100==0$ :

eigenvalues_ev[s] = ev $*$ au $2 \mathrm{ev} \# \mathrm{eV}$

\# second derivative

derivative $2=$ (eigenstates_au $[\mathrm{s}][:-2]-2$

eigenstates_au $[\mathrm{s}][1:-1]+1$

eigenstates_au[s][2:]) /

psi $=$ eigenstates_au $[\mathrm{s}][1:-1]$

$\#\langle$ Psi|H|Psi $>$ 
367 \#

derivative $2+1$

$368 \#$

$\left.z_{-} a u[1:-1]\right)$

$369 \#$
$370 \#$
$371 \quad \#$

au 2 ev \# ev

$$
p_{-} h_{-} p=\operatorname{simps}(\operatorname{psi} \cdot \operatorname{conj}() *(-0.5 *
$$

$$
\text { V_shifted }[1:-1] * \text { psi), }
$$

\# divide por <Psi|Psi>

p_h_p / = A **2

eigenvalues_ev[s] $=\left(p_{-} h_{-} p \cdot r e a l+\right.$ shift $) *$

372

373

374

375

376

377

378

379

380

381

382

383

384

385

386

387

\# descobre se é repetido

eigenvalues $=n p \cdot \operatorname{array}([e v$ for $e v$ in $\backslash$

eigenvalues_ev ।

if ev $!=0.0]$ )

eigenvalues $=$

eigenvalues [eigenvalues.argsort()]

golden_ones $=[0]$

for $i$ in range(eigenvalues.size):

\# drop repeated and unbounded states

pres $=$

np.abs (eigenvalues [i]/eigenvalues [i-1]-1)

if $i=0$ or pres $<0.1 ।$

or eigenvalues [i] >np.max ( $\left.v_{-} e v\right)$ :

continue

golden_ones.append (i)

if len(golden_ones) < len(eigenvalues):

forecast_diff $=$ forecast $[-1]$ -

forecast $[-2]$

388

389

390

forecast_diff)

391

392

393

forecast_max $=\max ($ forecast $)$

forecast.pop(s)

forecast.append (forecast_max +

$$
s-=1
$$

break

precisions [s] = np.abs (1-eigenvalues_ev[s] /

1

eigenvalues_ev_ana[s])

395

if (iterations and counters $[\mathrm{s}]$ >=

396

397

398

399

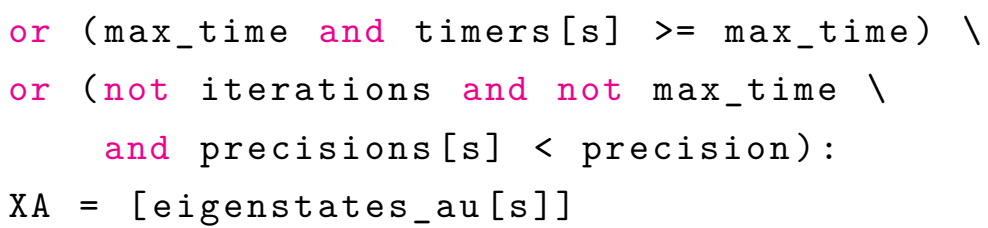


400

401

402

403

404

405

406

407

408

409

410 \#

411 \#

412 \#

413

414

415

416

417

418

419

420

421

422

423

424

425

426

427

428

429

430

431

432

433

434

435

436

437

438

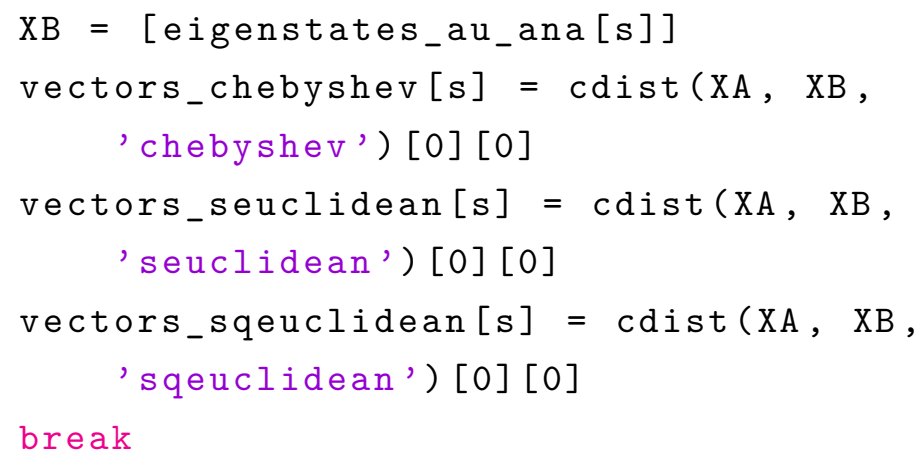




\section{B.6 Poço Quântico}

Classe utilizada para simular um poço quântico.

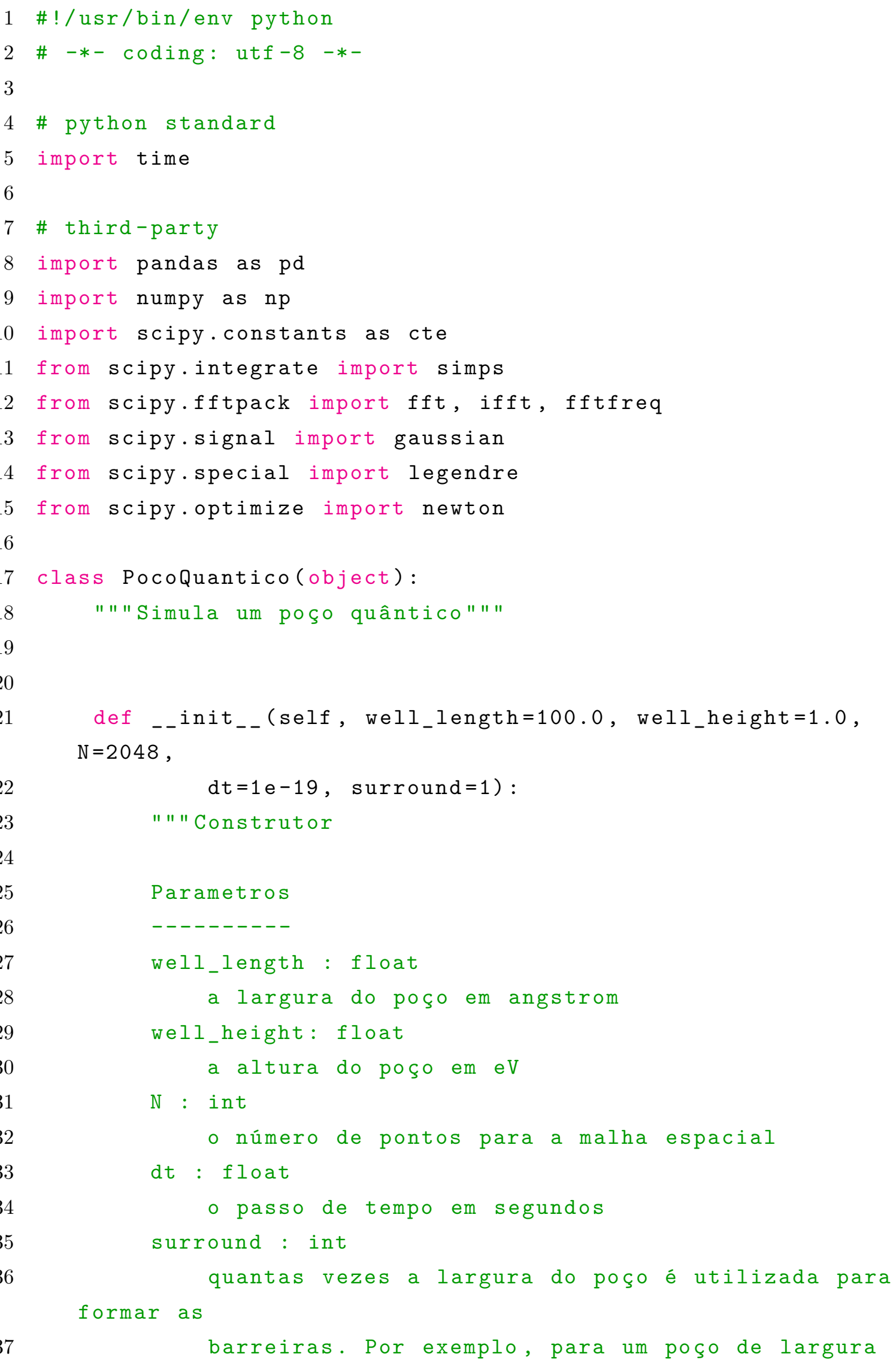


38

39

40

41

42

43

44

45

46

47

48

49

50

51

52

53

54

55

56

57

58

59

60

61

62

63

63

64

65

66

67

68

69

100.0 Angstrom, surround=1 significa que será

adicionado ao

sistema mais 100.0 Angstrom de cada lado, constituindo um

sistema de largura total 300.0 Angstrom. Para o

mesmo poço,

surround=2, seria adicionado ao sistema 200.0

Angstrom de

cada lado, totalizando um sistema de 500.0 Angstrom " " "

\# unidades atômicas

self.au_l = cte.value('atomic unit of length')

self.au_t = cte.value ('atomic unit of time')

self.au_e = cte.value ('atomic unit of energy')

\# outras constantes de interesse

self.ev = cte.value('electron volt')

self.au2ang = self.au_l / 1 e-10

self.au2ev = self.au_e / self.ev

\# massa efetiva constante

self.m_eff $=1.0$

\# cria um dispositivo

self.set_device(well_length, well_height, $N$, dt, surround )

def set_device(self, well_length=100.0, well_height=1.0, $\mathrm{N}=2048$,

(1)

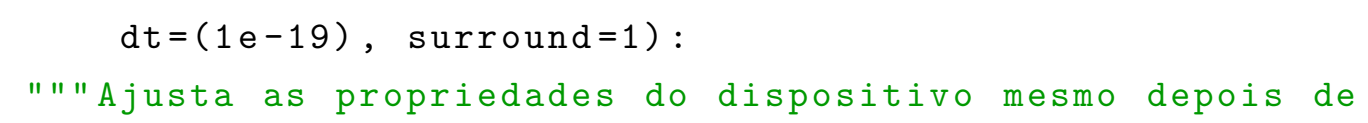
criado

Parametros

---------

well_length : float

a largura do poço em angstrom

well_height: float

a altura do poço em eV

$\mathrm{N}$ : int

- número de pontos para a malha espacial 


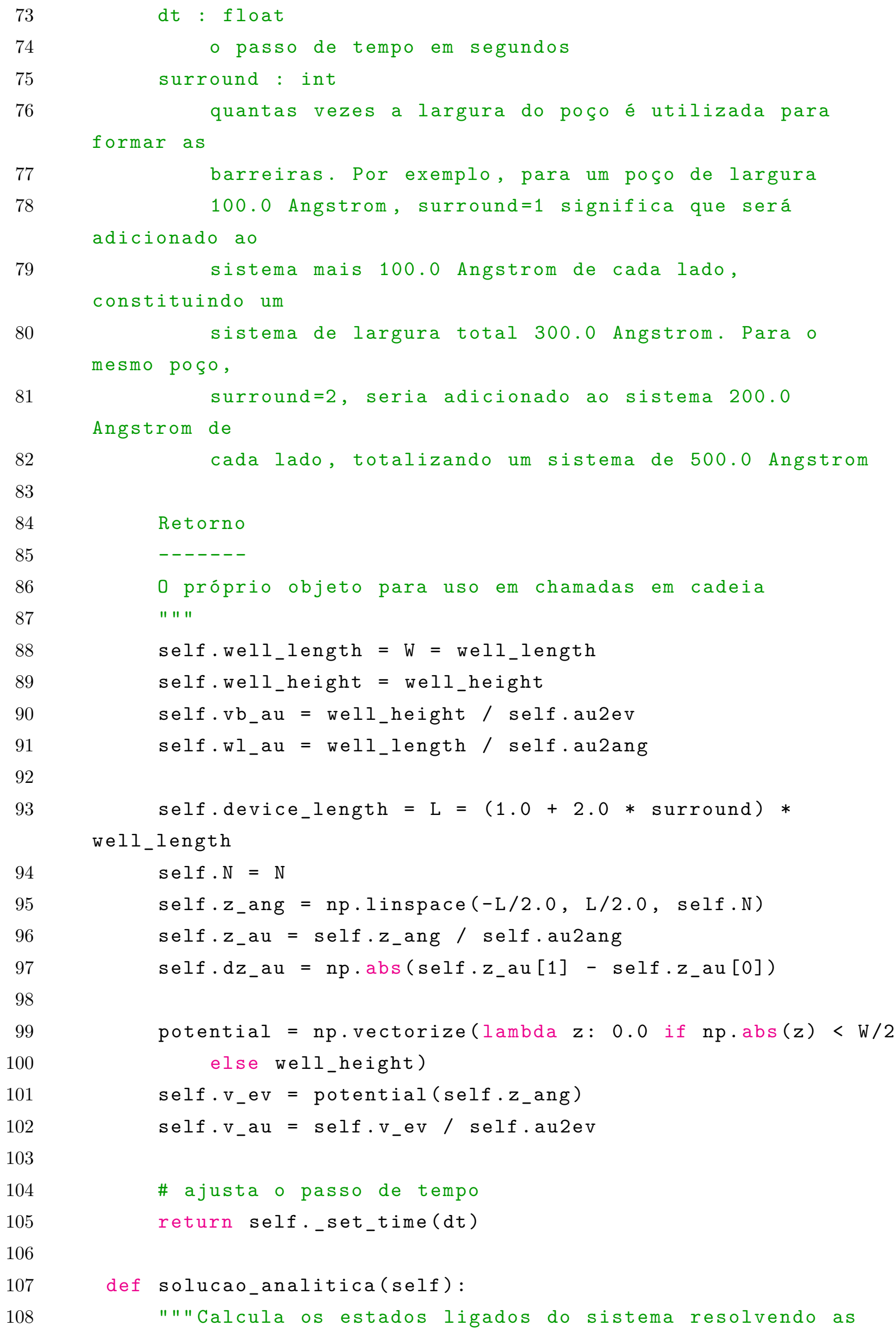


200

201

202

203

208

209

210

211

212

213

214

215

216

217

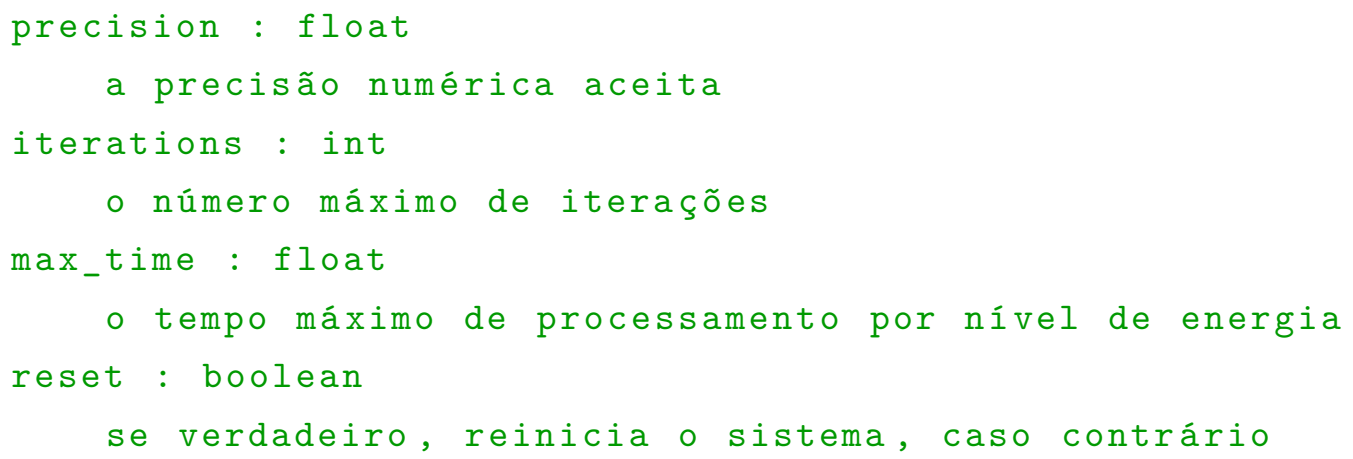

Retorno

0 próprio objeto para uso em chamadas em cadeia " " "

\# seleciona o modo tempo imaginário

self ._set_time (imaginary=True)

analytic_values = self.solucao_analitica()

if $n \max$ :

$\operatorname{nmax}=\min \left(\operatorname{nmax}, \quad l e n\left(\operatorname{analytic} \_\right.\right.$values $\left.)\right)$

else:

$n \max =\operatorname{len}($ analytic_values $)$

if reset or not hasattr(self, 'eigenvalues') :

\# inicializa os autovalores como zero

self.eigenvalues $=n p \cdot \operatorname{zeros}(n \max )$

short_grid = np.linspace $(-1,1, \operatorname{self} . N)$

\# inicializa os autovetores como polinômios de

legendre

\# multiplicados por uma gaussiana

$g=\operatorname{gaussian}(\operatorname{self} . \mathrm{N}, \operatorname{std}=\operatorname{int}(\operatorname{self} . \mathrm{N} / 100))$

self.eigenstates $=$

$n p \cdot \operatorname{array}([g *$ legendre (i) (short_grid)

for $i$ in range (nmax)], dtype=np. complex_) 
221

222

223

224

225

226

227

228

229

230

231

232

233

234

235

236

237

238

239

240

241

242

243

244

245

246

247

248

249

250

251

252

253

254

255

256

\# estado

self. counters $=n p \cdot z \operatorname{zeros}(n \max , d t y p e=n p \cdot$ int 32$)$

\# os timers vão salvar o tempo que cada estado leva self.timers $=n p \cdot \operatorname{zeros}(n \max )$

\# salva a precisão obtida em cada autoestado self.eigenvalues_precisions = np.zeros (nmax)

\# salva a precisão obtida em cada autovalor self.eigenstates_precisions $=$ np.zeros (nmax)

\# split step

for $s$ in range (nmax):

while True:

\# começa a contar o tempo aqui

start_time $=$ time.time ()

self.eigenstates $[\mathrm{s}]=1$

self.evolution_operator (self.eigenstates [s])

self.counters $[s]+=1$

\# gram-schmidt

for $m$ in range( $s)$ :

proj $=\operatorname{simps}($ self.eigenstates $[s] * \backslash$ self.eigenstates $\left.[\mathrm{m}] \cdot \operatorname{conj}(), \operatorname{self} . z_{-} a u\right)$ self.eigenstates [s] $-=\operatorname{proj} *$

self.eigenstates [m]

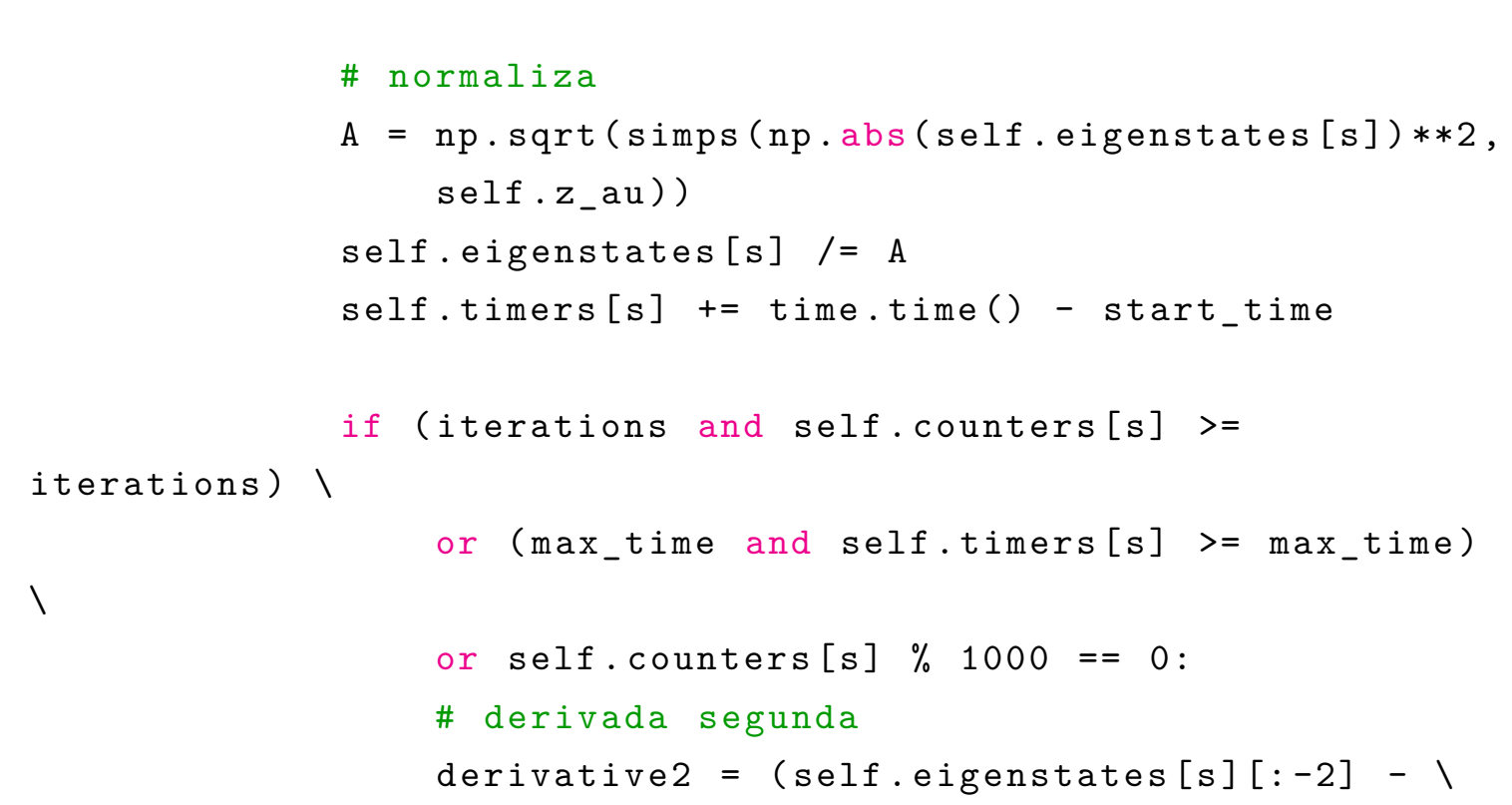


260

261

262

263

264

265

266

267

268

269

270

271

272

273

274

275

276

277

278

279

280

281

282

283

284

285

self.eigenvalues [s],

286

287

analytic_values [s],

self. counters $[s])$ )

288

289

290

291

derivative2 / ।

self.au2ev \# eV

$-1$

analytic_values $[\mathrm{s}]$ )

iterations) ।

max_time) ।

precision) ।
$2 *$ self.eigenstates $[\mathrm{s}][1:-1]+1$

self.eigenstates[s][2:]) / self.dz_au**2

psi $=\operatorname{self}$. eigenstates $[s][1:-1]$

\#<Psi|H|Psi>

$p_{-} h_{-} p=\operatorname{simps}(\operatorname{psi} \cdot \operatorname{conj}() *(-0.5 *$

$$
\text { self.m_eff + self.v_au[1:-1]*psi), । }
$$$$
\text { self. } z_{-} \text {au }[1:-1] \text { ) }
$$

\# divide por <Psi|Psi>

$\# p_{-} h_{-} p \quad /=A * * 2$

value_before = self.eigenvalues[s] or 1.0

self.eigenvalues $[s]=p_{-} h_{-} p \cdot r e a l *$

self.eigenvalues_precisions $[s]=n p \cdot a b s(1.0$

if (iterations and self.counters [s] >=

or (max_time and self.timers $[\mathrm{s}]>=$

or (not iterations and not max_time and $\backslash$ self.eigenvalues_precisions [s] <

or self.counters[s] > 300000: \# hard code

print (" " "Energy $[\{\}]$ :

Numeric $=\{: .10 e\}$

Analytic $=\{: .10 e\}$

iterations $=\{\} "$ " . format (s, 
292

296

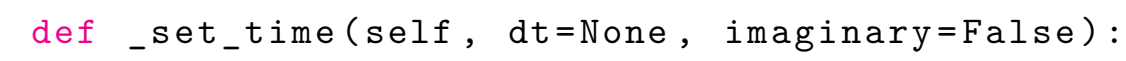

6

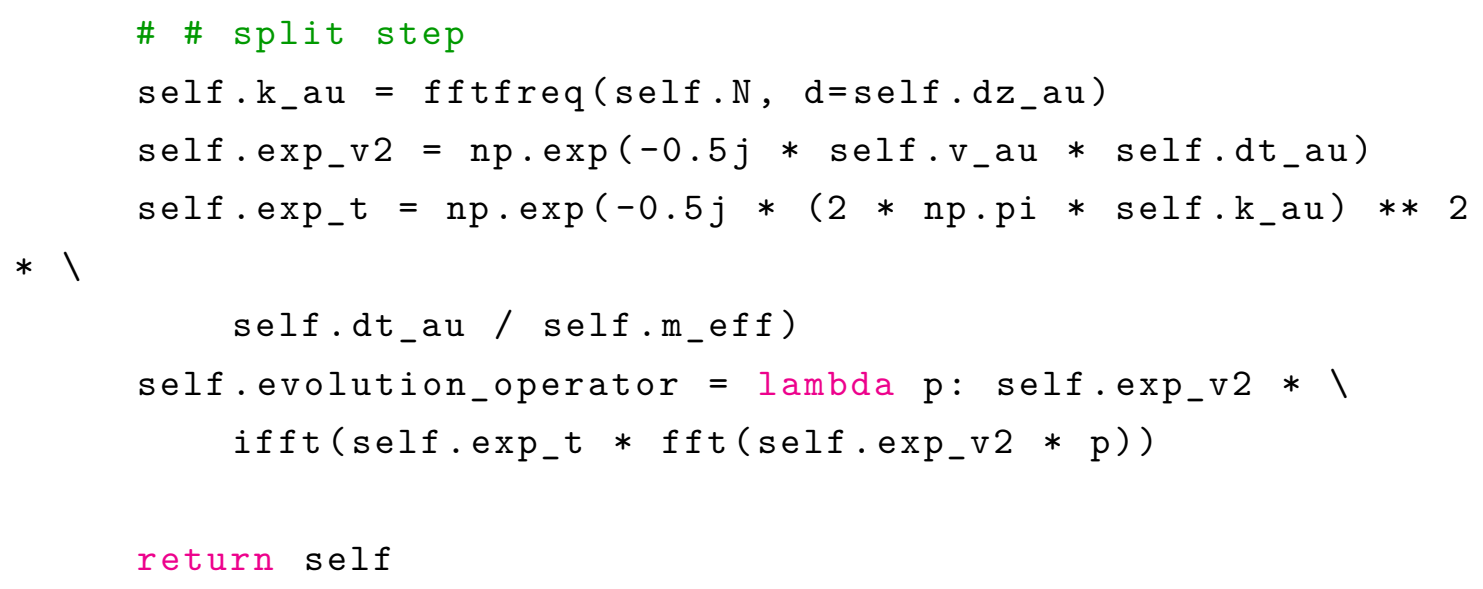

\section{B.7 Potencial do Poço com Dupla Barreira}

Funções utilizadas para gerar o potencial de poço quântico com dupla barreira. 


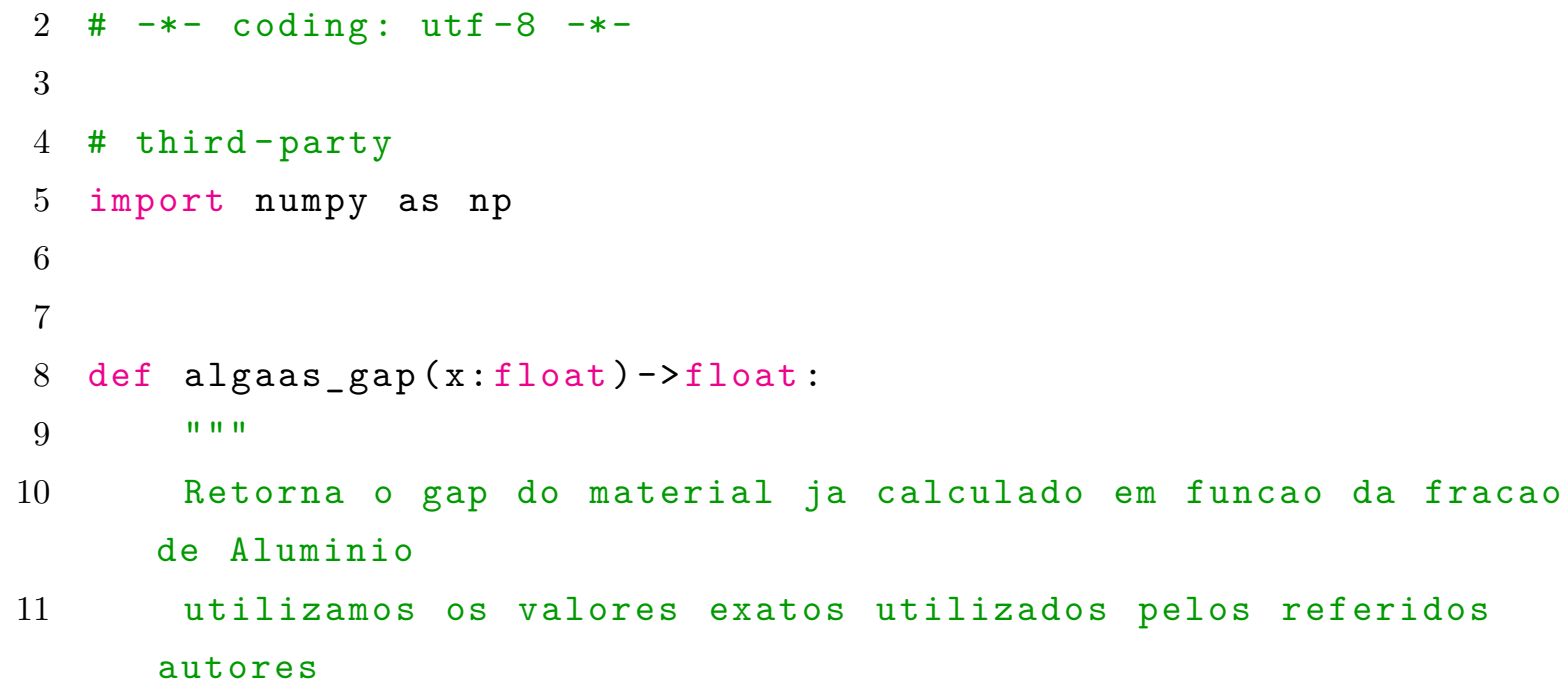




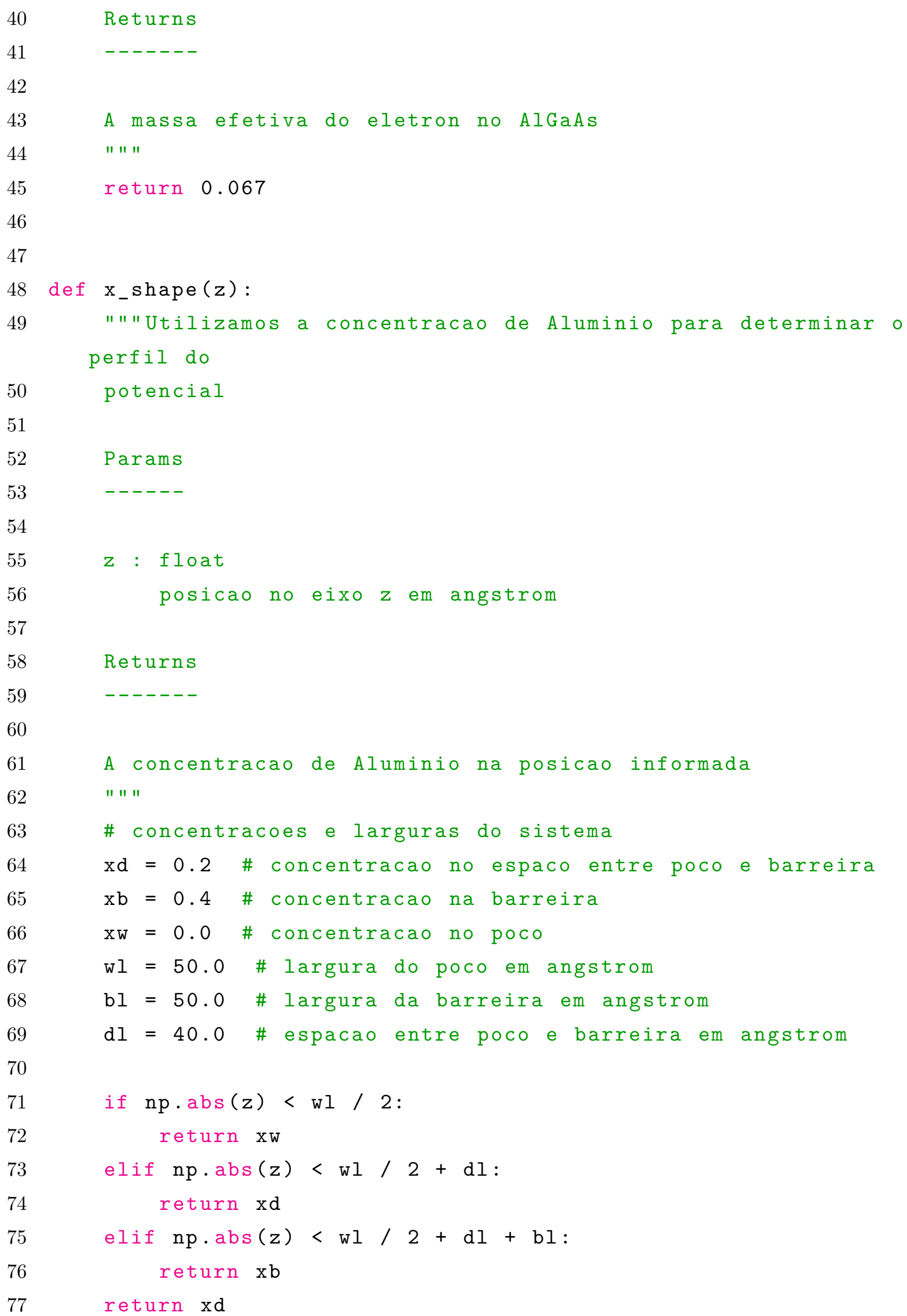

